\title{
Triangle Momentum
}

Steven Brasell

Supervisors: Budhi Surya, Leigh Roberts and Yigit

Saglam

\section{VICTORIA UNIVERSITY OF WELLINGTON}

Te Whare Wānanga o te Ūpoko o te Ika a Māui

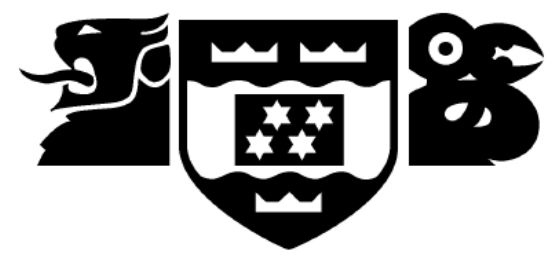

School of Mathematics and Statistics

Te Kura Mātai Tatauranga

A thesis

submitted to the Victoria University of Wellington in fulfilment of the requirements for the degree of Master of Science

in Stochastic Processes in Finance and Insurance.

Victoria University of Wellington 



\begin{abstract}
This research investigates the breakout of security prices from periods of sideways drift known as Triangles. Contributions are made to the existing literature by considering returns conditionally based on Triangles in particular terms of how momentum traders time positions, and by then using alternative statistical methods to more clearly show results. Returns are constructed by scanning for Triangle events, and determining simulated trader returns from predetermined price levels. These are compared with a Naive model consisting of randomly sampled events of comparable measure. Modelling of momentum results is achieved using a marked point Poisson process based approach, used to compare arrival times and profit/losses. These results are confirmed using a set of 10 day return heuristics using bootstrapping to define confidence intervals.
\end{abstract}

Using these methods applied to CRSP US equity data inclusive from years 1960 to 2017, US equities show a consistent but weak predictable return contribution after Triangle events occur; however, the effect has decreased over time, presumably as the market becomes more efficient. While these observed short term momentum changes in price have likely been compensated to a degree by risk, they do show that such patterns have contained forecastable information about US equities. This shows that prices have likely weakly been affected by past prices, but that currently the effect has reduced to the point that it is of negligible size as of 2017. 



\section{Acknowledgements}

While this thesis took considerable effort, this still seems like depositing an invisibly thin hair on a vast mountain of other people's knowledge. I am grateful for contributions from the various teachers who have taught me over the years, and in no particular order:

Credit goes to Budhi Surya upon whose mathematical skill and guidance much of this work is based.

Thanks to Leigh Roberts, whose contribution of longer term guidance has enabled the pursuit of this subject and without whom this thesis would never have been completed. It has been an innovative learning experience to discuss ways of viewing financial markets from new perspectives and I am grateful for someone to not only take the chance on an obscure idea but to push it forward.

I am grateful to be taught by Professor Estate Khmaladze. By being caught up in Estate's deep probabilistic insights, a window opened into another world that I did not know existed. 



\section{Contents}

1 Introduction $\quad 1$

1.1 Triangles .............................. 2

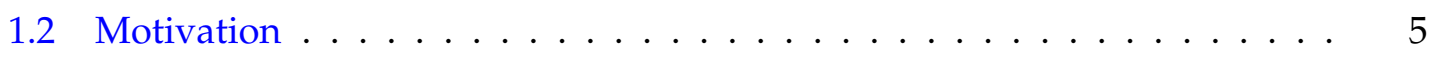

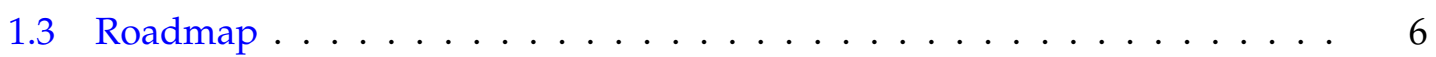

2 Literature $\quad 7$

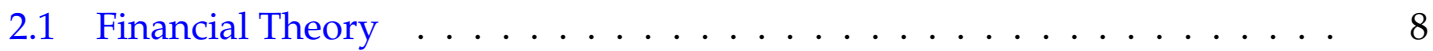

2.1.1 Market Efficiency and Random Walk . . . . . . . . . . . 8

2.1.2 Behavioural Finance . . . . . . . . . . . . . . . . . 9

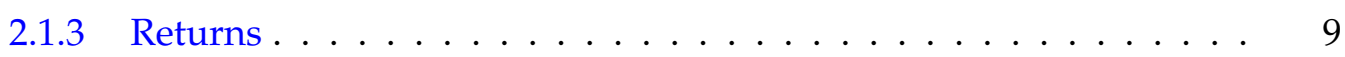

2.2 Security Trading . . . . . . . . . . . . . . . . . . . . . . 11

2.2.1 Back-testing Issues . . . . . . . . . . . . . . . . . . . . . . . . . . . . . . . . . . . 13

2.2.2 Technical Pattern Trading . . . . . . . . . . . . . . . . . . 15

2.3 Research Methods . . . . . . . . . . . . . . . . . . . . . 17

2.4 summary . . . . . . . . . . . . . . . . . . . . . . . . 18

3 Method 20

3.1 Triangle Trades . . . . . . . . . . . . . . . . . . . . . . 21

3.1.1 TTop Up (Triangle Top Break Up) . . . . . . . . . . . . . . . . . . 23

3.1.2 TTop Down (Triangle Top Break Down) . . . . . . . . . . . . . . 23

3.1.3 TBot Up (Triangle Bottom Break Up) . . . . . . . . . . . . . . . 23

3.1.4 TBot Down (Triangle Bottom Break Down) . . . . . . . . . . . . . . 24

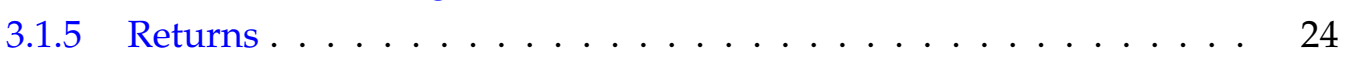

3.2 Triangle Detection . . . . . . . . . . . . . . . . . . 27

3.2.1 Improvements on Literature . . . . . . . . . . . . . . . . 28

3.2.2 Grid Map-Reduce . . . . . . . . . . . . . . . . . . . . . . . . 30

3.2 .3 Rolling Window Scan . . . . . . . . . . . . . . . . . 31

3.2.4 Detection Algorithm . . . . . . . . . . . . . . . . . . . . 32

3.3 Naive Trades . . . . . . . . . . . . . . . . . . . . . . 37

3.4 Results Modelling . . . . . . . . . . . . . . . . . . . . . 40

$4 \quad$ Structural Model $\quad 42$ 
4.1 Brownian Motion First Passage Derivation . . . . . . . . . . . . . . 44

4.2 Brownian Motion First Passage Estimation . . . . . . . . . . . . . . . . 49

4.3 Surface Exploration . . . . . . . . . . . . . . . . . . . . 51

4.4 Euler Estimation Baseline . . . . . . . . . . . . . . . 51

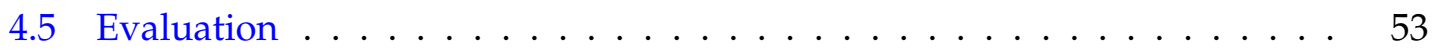

5 Intensity Model

5.1 Base Intensity Model . . . . . . . . . . . . . . . . . . . 58

5.2 Estimation Considerations . . . . . . . . . . . . . . . . . . . . . 61

5.3 Generalized Extreme Value Base Model . . . . . . . . . . . . . . . . . 62

5.3 .1 Parameter Estimation . . . . . . . . . . . . . . . 64

5.4 Goodness of Fit . . . . . . . . . . . . . . . . . . . . 65

5.4.1 Cumulative Deviations Test Construction . . . . . . . . . . . . 66

5.4 .2 Goodness of Fit Test Strategy . . . . . . . . . . . . . . . . . 67

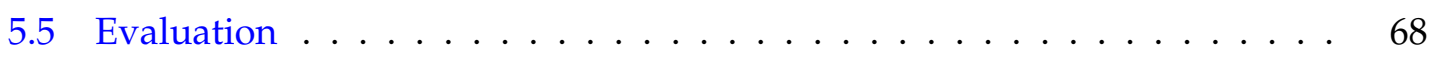

$\begin{array}{lll}6 & \text { Effect Size Heuristics } & 70\end{array}$

7 US Equity Results $\quad 73$

$7.1 \quad$ A Tail of TTop $\mathrm{Up} \ldots \ldots \ldots \ldots$. . . . . . . . . . . . . . . . . . . . . . . . . . . . . . . . 74

7.2 Intensity Model . . . . . . . . . . . . . . . . . . . . 78

7.3 Effect Size Heuristics . . . . . . . . . . . . . . . . . . . . . . . . 94

8 Discussion $\quad \mathbf{1 0 0}$

8.1 Alternative Result Evaluation Models . . . . . . . . . . . . . . . . . 102

8.1 .1 Intensity Cox Sum . . . . . . . . . . . . . . . . . 103

8.2 Extensions . . . . . . . . . . . . . . . . . . . 106

8.2.1 General Pattern Framework . . . . . . . . . . . . . . . . . . . 106

8.2.2 Portfolio Profit and Loss Analysis . . . . . . . . . . . . . . 107

$\begin{array}{lll}9 & \text { Conclusion } & 109\end{array}$

$\begin{array}{ll}\text { A Terms and Abbreviations } & \mathbf{1 1 0}\end{array}$

B US Equity Data $\quad \mathbf{1 1 2}$

B.1 Exploratory Statistics . . . . . . . . . . . . . . . . . . . . . . . . . 112

$\begin{array}{ll}\text { C GBPUSD Trial } & \mathbf{1 1 6}\end{array}$

C.1 Spot Price Data . . . . . . . . . . . . . . . . . . . . . . . . 117

C.2 Exploratory Statistics . . . . . . . . . . . . . . . . . . . . . 118

C.3 Structural Approach . . . . . . . . . . . . . . . . . . . . . . 120

C.3.1 Surface Exploration . . . . . . . . . . . . . . . . . 121 
C.4 Intensity Approach . . . . . . . . . . . . . . . . . . . . . 126

C.5 Discussion . . . . . . . . . . . . . . . . . . 130

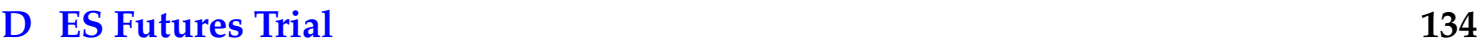

E Effect Size Heuristics Bootstrap Code 136 


\section{List of Figures}

1.1 Triangle Wave . . . . . . . . . . . . . . . . . . 1

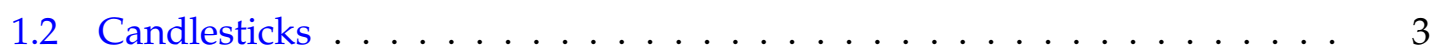

1.3 ES Triangle bottom with prior trend and break down . . . . . . . . . 3

1.4 Triangle with apparent surrounding noise . . . . . . . . . . . . 4

2.1 Resistance Breakout Example . . . . . . . . . . . . . . . . . . . . 13

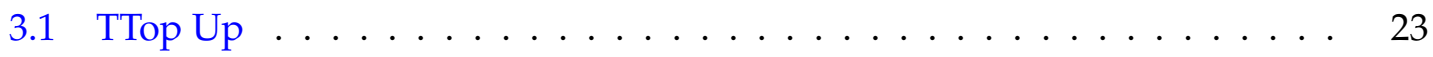

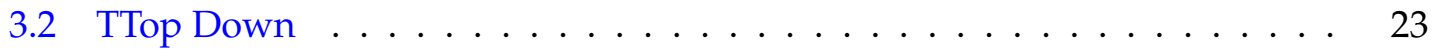

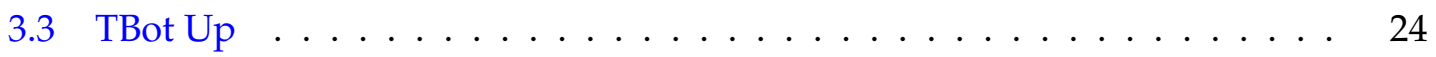

3.4 TBot Down . . . . . . . . . . . . . . . . . . . . . . . 24

3.5 XYZ Equity TBot Down Trade Example . . . . . . . . . . . . . . . . . . . 26

3.6 Triangle Class Diagram . . . . . . . . . . . . . . . . . . . 33

3.7 TBot Smoothness and Alignment Detection Example . . . . . . . . . . 33

4.1 Brownian Motion First Passage Example . . . . . . . . . . . . . . . . . . 43

4.2 Typical GBPUSD Brownian Motion First Passage Time $h=12$ (pips) . . . 49

4.3 TBot Up Constrained Brownian Motion 2D First Passage Distribution $\mu=$

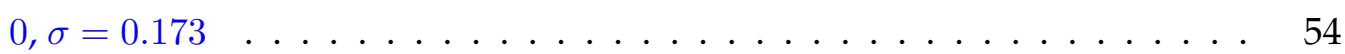

4.4 TBot Up Constrained Smoothed Naive (Heat) vs Brownian Motion (Terrain) $\sigma=0.173 \ldots \ldots \ldots \ldots \ldots \ldots$. . . . . . . . . . . . . . . . . . . 54

4.5 Constrained Smoothed Naive (Terrain) vs Triangle (Heat) . . . . . . . . . . 55

5.1 TBot Up Intensity Distribution Profile $h_{\sigma} \in[0.0035,007) \ldots \ldots \ldots$

7.1 TTop Up 2D Distribution Over $h_{\sigma} \in(0,10], \tau \in(0,60] \ldots \ldots \ldots \ldots$

7.2 TTop Up In Sample 1988-1998 Triangle GEV Intensity vs Empirical Triangle

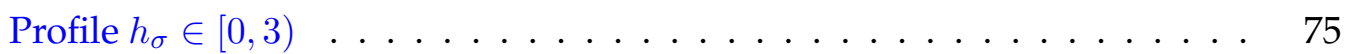

7.3 TTop Up In Sample 1988-1998 Triangle GEV Intensity vs Empirical Triangle

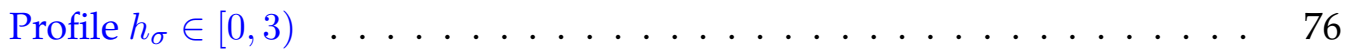

7.4 TTop Up 1988-1998 Triangle GEV Intensity vs 1998-2008 Forward Empirical Triangle Profile $h_{\sigma} \in[0,3) \ldots \ldots \ldots$. . . . . . . . . 77

7.5 Triangle Intensity vs Naive Empirical TTop Up Profile $h_{\sigma} \in[0,3) \quad \ldots \ldots$ 
7.6 Triangle Intensity vs Naive Empirical TTop Up Profile $h_{\sigma} \in[3,6) \quad \ldots . .88$

7.7 Triangle Intensity vs Naive Empirical TTop Down Profile $h_{\sigma} \in[0,3) \ldots . \quad 88$

7.8 Triangle Intensity vs Naive Empirical TTop Down Profile $h_{\sigma} \in[3,6) \ldots . . \quad 89$

7.9 Triangle Intensity vs Naive Empirical TBot Up Profile $h_{\sigma} \in[0,3) \ldots \ldots . \quad 90$

7.10 Triangle Intensity vs Naive Empirical TBot Up Profile $h_{\sigma} \in[3,6) \ldots . .91$

7.11 Triangle Intensity vs Naive Empirical TBot Down Profile $h_{\sigma} \in[0,3) \ldots . .992$

7.12 Triangle Intensity vs Naive Empirical TBot Down Profile $h_{\sigma} \in[3,6) \ldots$

8.1 Intensity Cox Sum Step Function ～. . . . . . . . . . . . . . . . . . 105

C.1 TBot Up Constrained Brownian Motion 2D First Passage Distribution $\sigma=$

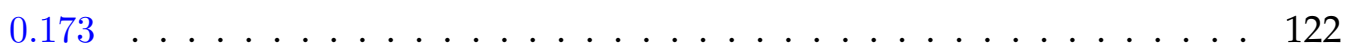

C.2 TBot Up Constrained Naive 2D First Passage Distribution . . . . . . . . . 123

C.3 TBot Up Constrained Triangle 2D First Passage Distribution . . . . . . . . 124

C.4 Contained Smoothed Naive(Terrain) vs Triangle (Heat) . . . . . . . . . . . 124

C.5 TBot Up Constrained Smoothed Naive(Heat) vs Brownian Motion (Terrain) $\sigma=0.173 \ldots \ldots \ldots \ldots \ldots \ldots$

C.6 TBot Up Intensity Distribution Profile $h_{\sigma}=[0,0.0035) \ldots \ldots \ldots \ldots \ldots$

C.7 TBot Up Intensity Distribution Profile $h_{\sigma}=[0.0035,007) \ldots \ldots$ 


\section{List of Tables}

4.1 GBPUSD Triangle Brownian Motion First Passage Estimates . . . . . . . . . . 50

4.2 GBPUSD Naive Brownian Motion First Passage Estimates . . . . . . . . 51

5.1 GBPUSD $h_{\sigma}$ Intensity Model Parameter Estimates . . . . . . . . . . . . 65

5.2 GBPUSD $h_{\sigma}$ Triangle Intensity Model In Sample Tests . . . . . . . . . . . . 68

7.1 US Equity GEV Model Parameter Estimates . . . . . . . . . . . . . . . . . . 79

7.2 US Equity In-Sample Triangle Cumulative Deviations Tests . . . . . . . . . 80

7.3 US Equity In Sample Naive Event Cumulative Deviations Tests . . . . . 82

7.4 US Equity Triangle Forward Cumulative Deviations Tests . . . . . . . . . . 83

7.5 Effect Size Heuristics Symbol Key . . . . . . . . . . . . . . . . . . . . . . . . 94

7.6 US Stock Triangle Momentum Heuristics . . . . . . . . . . . . . . . . . . . 94

7.7 US Equity Naive Event Heuristics ～. . . . . . . . . . . . . . . . . . . 97

A.1 General Terms and Abbreviations . . . . . . . . . . . . . . . . . 110

A.2 Return Terms . . . . . . . . . . . . . . . . . . . . . . . . . 110

B.1 US Equity Triangle Summary Statistics . . . . . . . . . . . . . . . . . 112

B.2 US Equity Naive Summary Statistics . . . . . . . . . . . . . . . . . . . . 114

C.1 GBPUSD Triangle Counts . . . . . . . . . . . . . . . . . . . . . . . . . . 118

C.2 GBPUSD Naive Event Counts . . . . . . . . . . . . . . . . . . . . . . 118

C.3 GBPUSD Triangle Stopping Times . . . . . . . . . . . . . . . . . . . . . 119

C.4 GBPUSD Naive Event Stopping Times . . . . . . . . . . . . . . . . . . . . 119

C.5 GBPUSD Triangle Mean Returns . . . . . . . . . . . . . . . . . . . . . 120

C.6 GBPUSD Naive Event Mean Returns . . . . . . . . . . . . . . . . . . . . . 120

C.7 GBPUSD Mean Return P-Values ．. . . . . . . . . . . . . . . . . . . . . 120

C.8 GBPUSD Triangle Brownian Motion First Passage Estimates . . . . . . . . 121

C.9 GBPUSD Naive Brownian Motion First Passage Estimates . . . . . . . . . 121

C.10 GBPUSD $h$ Intensity Section Counts . . . . . . . . . . . . . . . . 128

C.11 GBPUSD $h$ Intensity Model Parameter Estimates . . . . . . . . . . . . . 129

C.12 GBPUSD $h$ Triangle Intensity Model In Sample Tests . . . . . . . . . . . . . 129

C.13 GBPUSD $h_{\sigma}$ Intensity Section Counts . . . . . . . . . . . . . . . . . . . . 129 
C.14 GBPUSD $h_{\sigma}$ Intensity Model Parameter Estimates . . . . . . . . . . . . 130

C.15 GBPUSD $h_{\sigma}$ Triangle Intensity Model In Sample Tests . . . . . . . . . . . 130

D.1 ES Triangle Counts . . . . . . . . . . . . . . . . . . . . . . . 134

D.2 ES Triangle Mean Returns ． . . . . . . . . . . . . . . . . . . . . . 134

D.3 ES Triangle Mean Return T-test P-Values . . . . . . . . . . . . . . . . . 135 


\section{Chapter 1}

\section{Introduction}

This research is concerned with testing, understanding and forecasting changes in security prices after a form of price congestion called a Triangle has occurred, where prices form a triangular shape as illustrated in Figure 1.1. The principle is to compare security prices following a Triangle with unconditional prices to determine the impact of Triangles on returns. More specifically the research goal is to determine if there are predictable changes in trading returns after Triangle patterns.

There is contention both within the trading industry and academic finance as to whether such pattern based trading methods are profitable. While there is a small amount of related research, there seems no significant evidence to say that such methods generate abnormal profits; and the emphasis is on detecting differences in returns using p-value based testing without aiming for deeper understanding. Typically academic finance

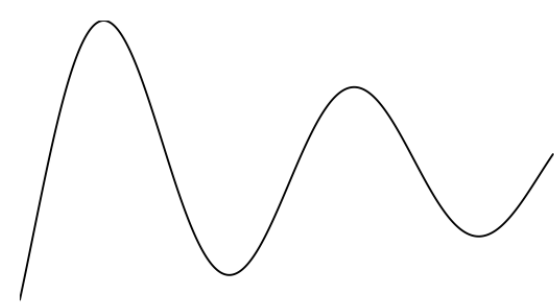

Figure 1.1: Triangle Wave insists that such methods do not produce abnormal returns; however the evidence is far from overwhelming. There is a reproducibility problem where some of the literature states that there is a significant effect of such patterns and some states otherwise. To delve further, this study takes an exploratory approach where I build an understanding of the mechanisms involved, based on an insider's view of how traders view the market, then objectively test the significance of results based on this. This approach has required developing methods to evaluate stock returns which are specifically suited to pattern based momentum trading.

The surprisingly sparse literature has utilised statistical methodology such that I am not able to make clear conclusions. So I spend considerable effort in what I hope provides a deeper understanding of the phenomena, which in conjunction with adjusted research methods should provide more convincing statistical results. I am able to do this with 
the advantage of proprietary trading industry knowledge and by restricting the study to Triangles, where other studies attempt to cover a larger range of patterns.

Security market regimes can be roughly divided into three: trending up, trending down or sideways. Generally the sideways movement is referred to as congestion or range bound, and it is a particular transition from this to a forecasted price that I am interested in. In order to narrow this to a defined range of detail, this study is based on periods where the prices of securities converge toward a particular focal region, which in the trading industry are commonly called Triangles, though there are a plethora of other variations and terms.

This simplifies the approach by narrowing the research to a very particular pattern of market congestion. The challenge is then to identify these regions in price algorithmically, measure the conditional price changes afterwards and explore their nature using stochastic models. I use trading industry terminology to refer to security price convergence and breakout in a market that is either trending up or trending down. Traders seeking to profit from Triangles use the notion that markets trend, then pause in a region of congestion, out of which some short term momentum can be forecast. This is the simplistic frame of reference used to understand changes in short term momentum.

As an overview, I attempt to model momentum by Structural and Intensity based methods; the Structural approach proves to have limitations and I choose to pursue the Intensity based approach more fully. To confirm the Intensity results I use a 10 day return heuristic with various bootstrapped confidence intervals. I later conclude that there is likely to be a small but significant predictable short term momentum effect after Triangles for US equities, but presumably as markets have become more efficient this effect has progressively diminished over the years 1960 to 2017.

\subsection{Triangles}

Often traders place huge emphasis on price, generally academics less so. When I worked on a trading floor as a software developer practically every single trader had numerous price charts tracking securities on up to eight screens each, yet when studying finance I did not once witness a single price chart on an academic computer. This is part of the reason I believe markets are affected by past prices, since traders use these in their decision making process to generate profits; it stands to reason that there may be some future trading significance based on these past prices. Possibly this is artificially induced by traders' behaviour, or traders are aware of repetitive market behaviour and are able to take advantage - there is some circularity. Although there are numerous ways in which traders derive meaning from plotting the price, in my experience usually these are simplistic, the chart being used simply as a reference to price levels of interest.

Clearly prices are not smooth on such charts as shown in Figure 1.1 for an ideal Triangle 
and additionally traders use high and low prices (not just closing prices), which this research takes into account. Price movements are then measured in terms of open, high, low and close prices of each measurement period (OHLC prices) and I display these price movements as candlestick charts. Candlestick charts in this study are displayed where white candles represent rising prices and red candles represent falling prices as displayed in 1.2. The bars represent the open to close price change, the vertical lines on either end of the bar represent the high and low price extremes within the period as displayed.

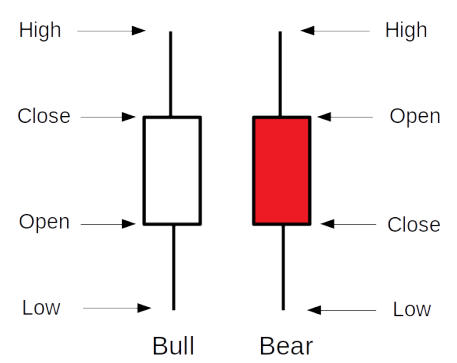

Figure 1.2: Candlesticks

With this in mind consider Figure 1.3 where ES (E-mini S\&P 500 Futures) appear to trend downwards to relative low point $E_{1}$ marked in blue, where price then converges to form a triangular shape including points $E_{1}$ to $E_{4}$ similar to the dampened wave example. Price then moves sharply downwards. Consider that at the blue $X$ mark the trader identifies the triangle pattern and waits for a move of price further downwards. The trader then sells short at the first green $X$ entry mark and buys at the next green $X$ to settle for a profit. This example represents the common idea behind how pattern based momentum traders trade breakout strategies. The idea is that traders can profit by being able to predict the short term changes in price after the triangle is formed, as the triangular shaped area marked in blue is escaped by price moving either downward or upward. I evaluate returns in this study based on similar simulated trading conditions.

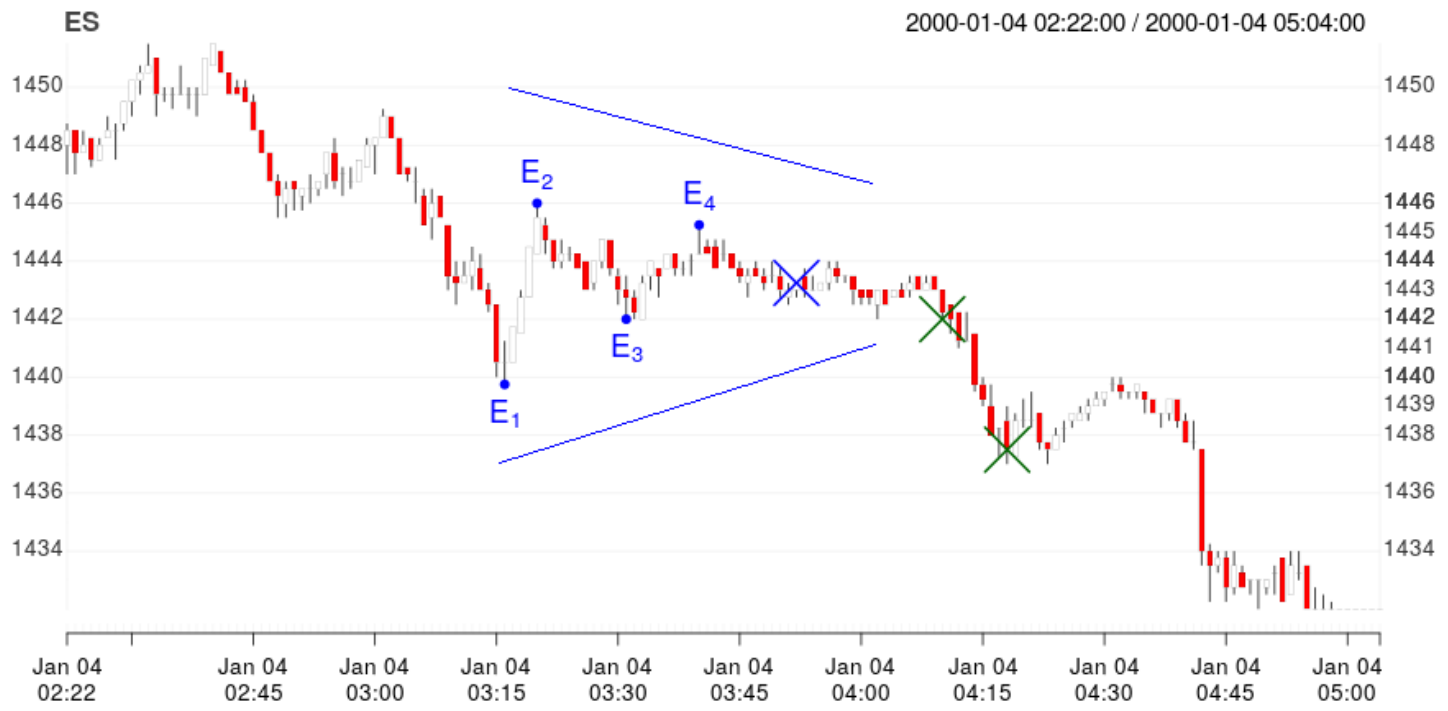

Figure 1.3: ES Triangle bottom with prior trend and break down

However this triangular shape is often not clear. In Figure 1.4 it is not so obvious that 
there is any pattern and the eye can pick out various triangular shapes such that it is not clear which is the correct one or more to pick. If we consider the one marked using points $E_{1}$ to $E_{4}$ it is also arguable whether there is a previous trend, or after the Triangle if there is any particular predictable future direction. Capturing what is and is not a valid Triangle is subjective, and it is consequently difficult to build an algorithm to detect patterns. These patterns are also easily reproduced using various stochastic processes, in a sense implying price congestion may be purely random or at least not due to any underlying fundamental events which might be ascribed to them. Further, Mandelbrot and Hudson (2004) makes the strong point that such repetitive patterns can be found commonly in purely random data that represents typical features of financial markets, yet which clearly have no underlying fundamental cause.

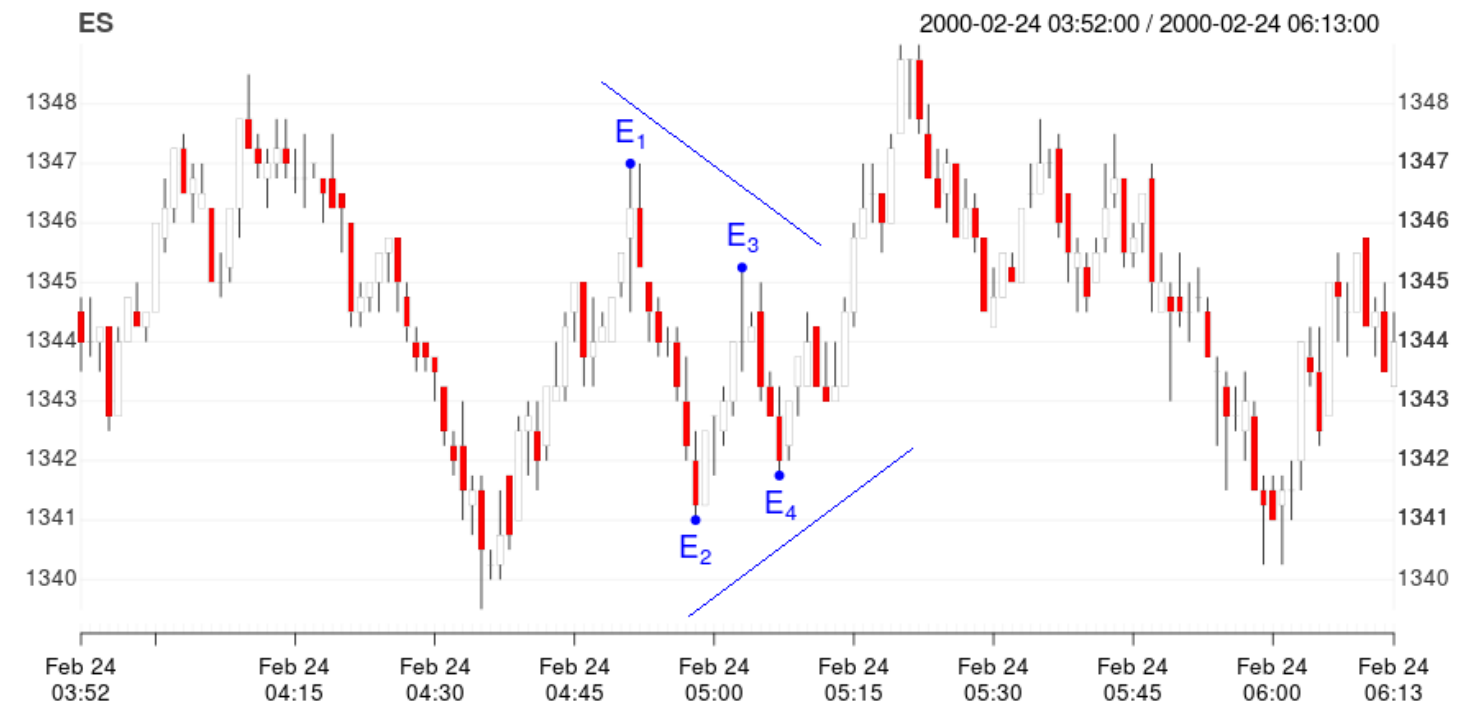

Figure 1.4: Triangle with apparent surrounding noise

Triangles are a well known phenomenon, and are frequently discussed in the news, captured by technical analysis concepts and anecdotally used by momentum traders to capture returns. However, despite being commonly discussed in the finance industry, this does not seem to be as widely researched as would be expected. Partly this is due to the need for programming skills; a second reason is the almost religious fervour with which finance academics refute such ideas, exacerbated by a misunderstanding as to how such methods are traded. While all manner of statistical measurements have been used to test for financial market inefficiencies, these often use simple mathematical tools to detect potential trading strategies rather than utilising custom built algorithms. Custom built algorithms are, I would argue, more adaptable to solving pattern based price movements.

Despite there being a few pattern based technical analysis papers referenced, for the most part I have chosen a different methodology. Much of this study draws on Lo et al. 
(2000), who shows how some pattern based returns deviate from classic Efficient Market Hypothesis theory. However it is difficult to draw clear conclusions as tests appear inconsistent and later research disputes his claims. Lo's technical patterns of Triangles, Wedges and Pendants are all some variation of a triangular shape. Curiously none of the literature regarding technical analysis investigates the specifics behind the practical trading of these patterns; rather they focus solely on conditioning on the patterns and statistically testing future standard periodic returns against the null hypothesis of market efficiency. These tests often appear inconclusive and generally lack a depth of an understanding of trading. I partly build off Lo et al. (2000) but I simulate how traders trade Triangles and develop alternative methods to understand the nature of price changes.

\subsection{Motivation}

For a period I worked on a trading desk for a London based bank as a software developer. Having worked alongside traders I had noticed some aspects of institutional trading which seemed common, but which were not well understood. One such common occurrence is the reference to past price highs and lows; and reference to periods of market stagnation.

Although references cannot be provided for such behaviour and these are not scientific observations, they provide some initial insight with which to explore ideas for more objective work; and it seems pragmatic to use these to tailor my research methods. Strictly speaking I have observed broader phenomena than Triangles; but Triangles seemed an easily identifiable subset, and as it happens there is some related research to draw on.

In contrast after this trading work, wanting to know more I studied finance where I was told repeatedly that what I had observed on the trading desk did not exist, that markets are efficient and excess profits cannot be generated from observing past prices. This seemed ironic since I had developed tools for traders who had long standing careers making excess returns. Observing this contrast and reading further academic studies warranted considering that there may be a misunderstanding and that common methods used to trade could be revisited with improved and more specific statistical research methods, but based on how institutional traders consider the markets.

So instead of analysing price as standard periodic returns, I use the movement from one price to another to create a more realistic return, using reasoning that it seems a trader would consider. Returns are then produced by simulating traders taking directional momentum positions after Triangle patterns based on reasoned entry and exit levels, and these are compared with returns generated without this potential timing advantage as a comparison. This is a relatively crude approach where I put aside many core 
financial beliefs, in order to freshly statistically test the validity of trading under what I believe are more realistic and rigorously considered research methods. The difficulty of taking this approach is that is requires the invention of a range of simple new approaches.

\subsection{Roadmap}

The research is organized as an empirical study of security prices with the view to both testing and understanding the nature of how prices change conditioned on Triangles. The literature survey includes the fundamentals of finance related to testing returns. This covers related trading concerns from a more industry related context and reviews the literature of pattern based technical analysis papers, discussing techniques that can be reused and where improvements can be made. I further comment on contributions or extensions to these under the Method chapter.

Price compression detection algorithms and methodology are detailed in the Method chapter and later chapters explore and develop stochastic process theory for comparison with price distributions. Incrementally using trial data, stochastic models are explored to understand the later changes in price with relevant tests used to validate the significance of returns. To validate the modelling a 10 day return heuristic is bootstrapped. The results for US equities are then summarized and conclusions discussed. Under this structure stochastic models are used not to model the time series as a whole but rather to understand the later changes in price after conditioning on triangles, but only on a short term basis. Numerous methods have been considered but discussion of relevant alternative methods is deferred to the Discussion section. 


\section{Chapter 2}

\section{Literature}

In this section, I review the current literature by firstly giving an outline of the relevant fundamentals of market based financial theory; secondly I outline industry practices of trading, particularly related to momentum trading; then I analyse trading pattern recognition, and give an overview of relevant research methods. Briefly justified under the financial theory section, stochastic models are explored more deeply in the later momentum analysis chapter where they are discussed in relation to trial data.

There is little in the way of direct research related to pattern based trading which would include Triangles, or at least could provide some relevant material, and where there is indirectly related work the research methods used are of questionable value. Lo et al. (2000) is an exception, with a creative exploration of pattern based technical analysis; this paper is particularly well thought out and does not seem to have been significantly extended by later literature. Partly because of the lack of literature, I draw on professional trading floor experience, conversations with traders, and trading books. This is done to gain some depth of insight into how real trading is done to simulate trading more realistically.

In order to compensate for the lack of directly relevant literature and to improve the depth of understanding, various research methodologies and statistical testing avenues are explored. Depending on whose view is expressed, research in social sciences has faced various reproducibility and research methodology issues. The finance literature appears to suffer from the same problems, and so I explore approaches taken in other disciplines to develop sound research methods. 


\subsection{Financial Theory}

\subsubsection{Market Efficiency and Random Walk}

Arguably a core area of research in market based finance is Efficient Markets Hypothesis (EMH). Efficient markets theory from Fama $(1970,1965)$ and others argue that security prices reflect all available information, and further work also implies that prices vary as random walk based processes (Fama, 1991). There has been various research validating and refuting EMH over time; those against often look for counterarguments to the Weak form of EMH based on finding market anomalies. For example a commonly used argument against EMH is that market prices (typically stocks) show long term trend momentum where there is significant evidence to suggest over a period of weeks or months that trending stocks tend to outperform non-trending stocks: Lo et al. (2000); Jegadeesh and Titman (1993); Lo and MacKinlay (1999). It is now well accepted, even by Fama, that trend momentum is a consistent market anomaly that should not exist according to the EMH (Fama and French, 1996). Another common anomaly is that of mean reversion (Fama and French, 1988), the theory being that autocorrelations of returns show predictable positive or negative components. These anomalies tend to show long horizon results that contradict EMH; so there is considerable counter argument that markets are not efficient. As Thorp (2017) points out it is not possible to prove a negative, so it seems of negligible value to argue the absolute truth of the EMH. Perhaps a more pragmatic view as presented by Lo (2017) is the Adaptive Markets Hypothesis (AMH), which suggests a less extreme version of EMH modified by more modern behavioural concepts that accepts a degree of efficiency based on principles of evolutionary competition. Lo's AMH provides a more reasonable context under which to construct hypothesis tests through selective advantage.

Fama makes several compelling arguments for $\mathrm{EMH}$, arguing that the majority of the anomaly literature is probably due to randomness and the bad model effect over long term horizons (Fama and French, 1988). According to Fama, long term market anomalies, often coming from the Behavioural Finance literature, can be reduced to insignificance by improving the models of market efficiency tested against. He argues that there is a 'bad model' effect under which testing with long term data is common. However with the data used in this study I alleviate this by using granular data over very short term horizons, for example a number of days as opposed to months or years. Over a short term horizon there is little chance of significant differences in return distribution due to model issues. This simplification can be used to avoid the difficulties of constructing complex multi-factor models to account for more long term effects such as drift.

Partly driven by $\mathrm{AMH}$, a more pragmatic view of EMH is that markets are 'fractionally' efficient, there being certain situations over the short term where market participants' may not be rational or abnormal returns can be obtained over short periods. However it 
is reasonable to assume that, given the considerable weight of literature, most of the time financial markets are efficient. Taking this more relaxed view seems reasonable in the light of the numerous trading businesses and literature demonstrating abnormal returns, but perhaps only under unusual circumstances.

It is the fractionally efficient view that I investigate, assuming that abnormal returns cannot usually be made. This more relaxed view means I cannot assume markets are absolutely efficient, which motivates carefully constructing an alternative research methodology based on competitive advantage. This has prompted a carefully tuned comparison of Triangle and Naive model returns, as discussed further in Sections 2.3 and 3.3.

\subsubsection{Behavioural Finance}

Though EMH arguably dominates the lens of financial market theory, behavioural finance has been challenging this role. Behavioural economics pioneered by Kahneman (2011) has taken a financial slant and provides a useful framework from which to evaluate some trading anomalies by challenging the role of standard utility based approaches. Papers such as Frankfurter and McGoun (2002) argue supporting these ideas while Fama (1998) and associates argue against. An overview of the various arguments can be found in Thaler (2005).

The difficulty with such research is that while providing useful motivations for anomaly exploration, behavioural finance theories are hard to validate without deep financial empirical data. It is hard to know the reasons for market participants behaviour without having specifically detailed information about their trading, and this is generally not available. Nonetheless concepts such as anchoring and herding from this literature provide useful motivations for designing research methods for short term momentum effects. While many traders may not know the relevant theory they purportedly make good use of behavioural concepts to make profits as seen in Brant (2011), and so these theories provide an attractive framework to discuss short term momentum trading.

Again Lo (2017) provides a comprehensive context for behavioural vs EMH arguments, motivating a fractionally efficient market for statistically testing models. Behaviourally it makes sense to consider competing survival based innovation to compare Triangle models with so called Naive models constructed as an alternative test case which does not require markets to be efficient (see Section3.3 for Naive model construction details).

\subsubsection{Returns}

There are some stylised facts for financial returns that are relevant as background, which are explained in detail by Taylor (2011) and are relevant to this study. These stylised facts are (paraphrased from Taylor): the distribution of equity returns are approximately symmetric but have high kurtosis relative to the normal distribution, indicating fat tails; 
the autocorrelations of returns are all close to zero; and that the autocorrelations of both absolute returns and squared returns are often positive for many lags (volatility clustering). Though these are often referenced with regard to daily stock returns, a similar rationale applies to currency markets.

For this particular study the symmetry of returns and volatility clustering are of interest, in that our simulated returns are not symmetric and there is some anecdotal possibility of predictable volatility. However in reviewing these stylised facts there seems a drastic reliance on periodic return distributions, where from experience and reviewing trading books it appears that in the discretionary trading industry few use such methods. Periodic returns refer to single period returns (often daily returns) where each period has return

$$
r_{i}=\frac{p_{i}-p_{i-1}}{p_{i-1}}
$$

where $p_{i}$ is the final price at time $i$. Since it is commonly assumed prices are log normally distributed then $\ln \left(1+r_{i}\right)$ is normally distributed since

$$
1+r_{i}=\frac{p_{i}}{p_{i-1}}=e^{\ln \left(\frac{p_{i}}{p_{i-1}}\right)}
$$

Comparatively, returns can be represented in continuous time as $d t \rightarrow 0$ where

$$
\frac{S_{t+d t}-S_{t}}{S_{t}}=\frac{d S_{t}}{S_{t}}=\mu d t+\sigma d B_{t}
$$

Periodic returns are computed and analysed in terms of their distribution, often modelled log normally, to gain insight into the nature of security markets. This is usually moved into continuous time and Itô processes are used to represent the structure of price changes as seen in (2.3) for Geometric Brownian motion. These forms of return represent mathematical conveniences, providing an assortment of well understood tools: see Duffie (2010) for a deeper assessment of these. This is the standard approach but there are various issues with this, as seen for example in Hudson and Gregoriou (2015). While the majority of these points are obvious to state, the main one is that actual returns do not consist of conceptualising price in these terms and so from a very raw perspective this paradigm may not be as useful as it initially appears.

In contrast, the trading industry back-tests simulating returns and measures actual returns based on particular entry and exit points to form return distributions which represent real gains and losses. In academic finance there seems an overwhelmingly strong herd perspective based on periodic returns that I would suggest clearly does not represent actual returns well as used in industry, and which may blind researchers to be over reliant on mathematics that is too abstracted from reality. This matter is 
complicated by issues of leveraged trading where short term momentum traders borrow to make leveraged bets for short holding periods, and this does not fit well within either the periodic return or the longer term investor holding period return as represented by

$$
r_{h}=\frac{\text { income }+p_{h+s}-p_{s}}{p_{s}}
$$

where $p_{s}$ is the value at the start of the holding period and income $+p_{h+s}$ is received the end of holding period value.

To avoid these issues and to more appropriately tailor my methods to leveraged short term momentum trading, I explore the use of stochastic processes to model raw price movements to fixed price levels, using very large sample sizes to make short horizon based conclusions, exploring the testing for abnormal returns in some depth without using periodic returns. Also I consider how to modify the holding period return to use standardised short term risk adjusted returns using $\mathcal{R}$ based modification as seen in Tharp (2008). This avoids the 'bad model' effect issues and allows simplified testing as longer term factors will have less effect, and so the incremental process of model selection is considerably less complex. The caveat is a potential lack of the ability to measure long term effects of Triangles; but momentum traders consider only short horizons and so this is of less interest. For a wide ranging discussion on the use of random walk and stochastic processes in the testing of abnormal returns see Malkiel (2003).

For reference and to clarify the use of alternative measures, a summary of returns terminology used in this study is seen in Table A.2.

\subsection{Security Trading}

Trading the secondary security markets is generally considered a short term affair consisting of a holding period intra-day or perhaps of a few days, whereas investing is generally considered a long term buy and hold scenario where usually analysis of fundamentals is given considerable attention or alternatively index tracking is done. Trading generalisations for this section can be found in books by Chan $(2013,2009,2016)$ and Carver (2015) who actively trade institutionally and provide transparent insights into their methods; while these are relatively well known, Section 2.2.2 contains more specific Triangle information and references. Using these books as context, short term trading generally falls under two types: mean reversion trading and momentum trading. Mean reversion trading is generally where pairs or more commonly baskets of securities are held, some long and some short, with the expectation of reversion to a mean. Trading is further generalised by traders either being quantitative (usually automated) or discretionary. The focus of this study is on momentum based 
discretionary traders, in particular focusing on level based Triangle trading.

Momentum trading uses the idea that certain market effects cause prices to continue in a predictable direction for a period of time or to a fixed level. Momentum trading broadly falls under the following types as characterised by Chan (2013):

- the persistence of futures roll return,

- slow diffusion of new information,

- forced security transactions of funds causing price pressure effects.

- high frequency trading.

- support and resistance.

Support and resistance is the category of momentum trading relevant to this research, which is where common local extrema are deemed significant to future price momentum. In this context momentum trading uses the notion of breakouts, where after the price moves beyond some preassigned level, there is a rapid change in price for a period of time or to another price level. This is part of the general category under which pattern based technical analysis falls.

Resistance refers to a level of price at which prices have reached a set of extremum from below, where sellers or buyers are willing to trade, potentially affecting the price. Support is defined as a set of price extrema from above where similarly it is expected there is a change in trading activity. Though there are various arguments as to which why and which way price will move that often contradict, the idea is that momentum traders who understand these effects are able to predict and profit in either direction. A price pushing through the resistance or support is called a 'break out'. Brant (2011) has published a diary of his professional trading which shows how pattern based support and resistance trading is done in practice using real audited trades to profit.

There is some evidence to suggest that returns generated by support and resistance have valuable information; for example (Raj and Thurston, 1996) gives a simple example of profitable trading range break out strategies being successful on the Hong Kong futures markets. A typical example of this method is visually demonstrated in Figure 2.1 where resistance forms a temporary barrier which once broken though seems to show sharp momentum to a higher price at the right edge of the orange line. Price momentum in this case refers to a large price move within a short amount of time. Statistical evidence as to how effective these are, however, seems arguable: Osler (2000) shows that significance of momentum from such levels is at best sporadic and so may be spurious. However support and resistance based methods are of interest because discretionary traders state they use such price levels to trade profitably, and so both literature and trading experience prompts further investigation which I detail in Section 2.2.2.

In the next few subsections I summarise various attributes of trading system testing and techniques which are useful best practices for researching trading strategies. These are 


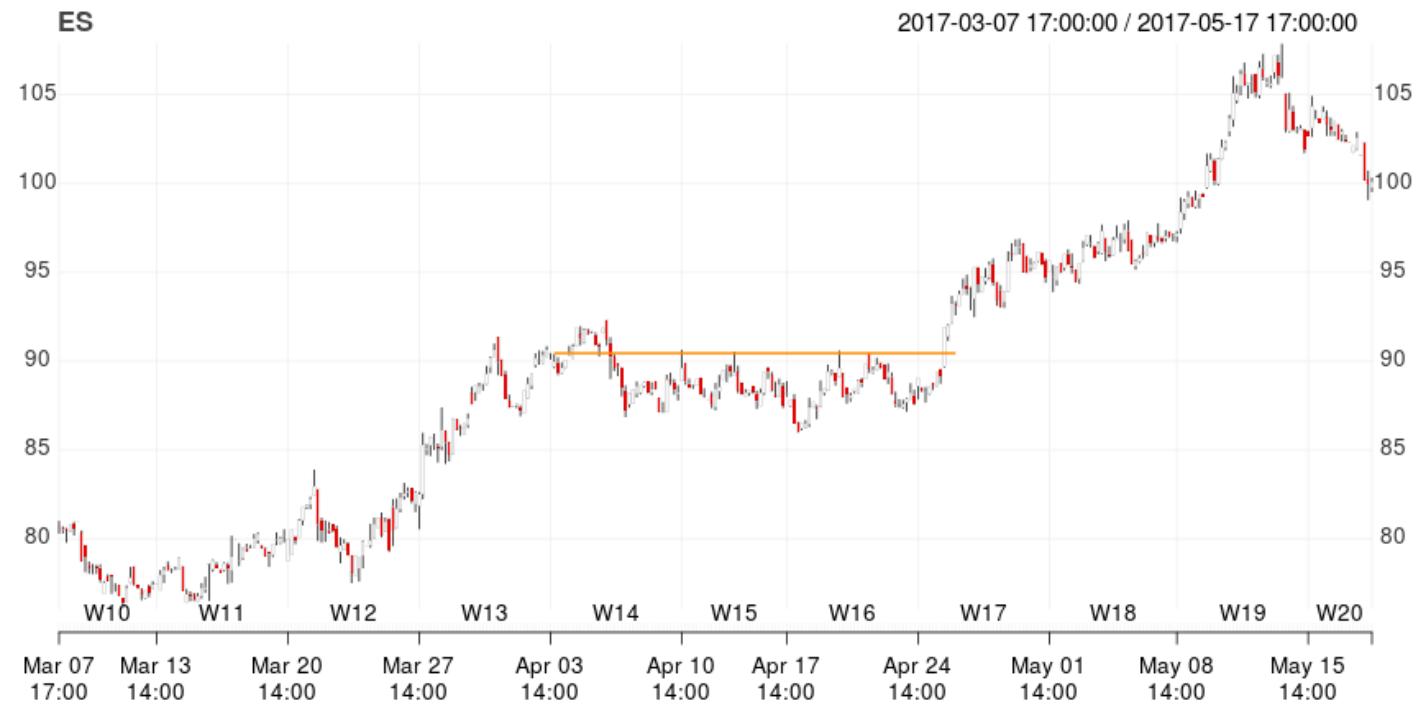

Figure 2.1: Resistance Breakout Example

often omitted from some trading based academic research, where their effects could lead to spurious conclusions. Lo and MacKinlay (1999) and Malkiel (2003) show that a large proportion of the literature to do with market anomalies are of questionable value, arguably due to inadequate research methods.

\subsubsection{Back-testing Issues}

In this thesis I simulate traders taking positions, which requires back-testing break out trading considerations. Back-testing involves running the trading strategy forward on historical data to capture and evaluate returns. For the purposes of this study this involves the following equity based back-testing considerations:

- look ahead bias,

- survivorship bias,

- data-snooping bias,

- transaction costs,

- short sale constraints.

\subsubsection{Look Ahead Bias}

A bias created by testing with forward knowledge which would not have been available during the period when forecasting; encountered where a researcher back-tests a trading strategy using future information to make predictions. This is avoided by only using data before the currently observed period in time and placing trades based on this prediction in the current time period or future time periods. 


\subsubsection{Survivorship Bias}

Survivorship bias is where data is used from surviving securities to predict past performance (type of look ahead bias). Take the case of the S\&P500 for example: if a researcher tested all current S\&P500 constituents for past returns, this testing inherently includes a knowledge that the stocks survived up to this point and which become large enough to be selected as part of the index. The test does not for example include bankrupt companies and so will report superior returns to a survivorship bias free dataset, and hence tests done will not be representative. This is generally avoided by using a survivorship bias free dataset such that past stocks are not chosen from later selected subsets of stocks.

\subsubsection{Data-Snooping Bias}

This is where inference or forecasting is done after having already viewed the data and hence having pre-existing insight which can be used to gain more significant results (further explored under research methods 2.3). Typically this is done where in-sample data is used iteratively with multiple models, which misleadingly can 'improve' model results. This is often done by a researcher who has previously modelled using the same data. Carver (2015) makes a detailed analysis of this, suggesting that data-snooping is virtually unavoidable but can be reduced significantly by only testing against real data as a last resort. I discuss avoidance of this problem in Chapter 3.

\subsubsection{Transaction Costs}

Trading transaction costs include the sum of commissions, transaction spread and slippage for each full trade consisting of the buy and sell. If the trade is taken using margin lending and held past the overnight cut-off time the cost of carry will also be included. Transaction spread is the difference between the Bid and Ask price, the calculation of which depends on the order types used (typically market, limit or stop orders with either a buy or sell). In general it is assumed that at the very least the difference between the Ask and Bid is effectively paid as part of the round trip trade costs. Slippage is the difference between the price the order is sent at compared with the price paid on the exchange (or broker internal cross match); again this depends on the order type, but may be significant if liquidity is absent.

\subsubsection{Short Sale constraints}

Many securities have time periods which restrict short sales or have difficulties with short selling due to a lack of liquidity or broker constraints. During financial crises governments may outright ban short selling and often rules are applied such as requiring an up-tick rule such as the one applied by the SEC from 1938 to 2007. Short selling also assumes the broker can locate a quantity of stocks that other customers have, to enable the short sell, and this may not be the case. Testing short selling needs to 
be considered on a case by case basis, and this is beyond the scope of this thesis.

\subsubsection{Technical Pattern Trading}

Previous research covering short term trading has paid some attention to technical analysis; but the focus has mainly been on simple quantitative measures such as moving averages, with little attention paid to support and resistance based strategies which are often referred to as pattern based technical trading. In this context pattern trading consists of price time charts forming certain geometric patterns which are said to forecast future price changes. The general area I had wanted to research consists of market price compression, where price forms periods of low volatility converging toward a focal price and traders are able to take advantage of predicting future volatility; typically this is not seen as technical analysis, but this is the only area of research relevant to the subject. To conform with other research I use the Triangle based idea and naming conventions. This results in some loss of generality but allows use of the limited research available. I avoid using many standard technical analyst ideas and language as they seem unnecessarily esoteric: see Murphy and Murphy (1999) for an overview of pattern based technical analysis methods. Generally under technical analysis, security financial information and news are considered embedded in the price, and so to simplify this study I will also use this convention and only consider price in order to constrain the scope of the research.

From an overall review of technical analyst literature, arguably the academic consensus is that quantitative technical analysis does not provide any information towards providing abnormal returns (Malkiel, 2003). While there is limited literature available on pattern based trading, which gives arguably interesting results, overall work in these fields appears inconclusive. Lo et al. (2000) is arguably one of the first papers to widely explore pattern based technical analysis systematically using algorithms. While there had been sporadic previous literature to validate patterns, particularly 'head and shoulders' in Osler et al. (1995), Lo raised the debate to a higher level. Later research however disputes Lo's findings. After this spate of work, further research appears to have generally been halted.

Lo makes the point that the language used by technical analysts is often unrecognisable to quantitative researchers and yet the underlying points are often quite similar. He and his coworkers argue that by converting the language of technical analysts into a common format and using algorithms, these visual patterns can be well tested. This language for Triangles is shown in (2.5) for a Triangle Top which is further elaborated for this study in Section 1.1. 


$$
\text { TTOP }=\left\{\begin{array}{l}
E_{1} \text { is a maximum } \\
E_{1}>E_{3}>E_{5} \\
E_{2}<E_{4}
\end{array} \quad \text { TBOT }=\left\{\begin{array}{l}
E_{1} \text { is a minimum } \\
E_{1}<E_{3}<E_{5} \\
E_{2}>E_{4} .
\end{array}\right.\right.
$$

In Lo's study patterns are detected within daily US equity price CRSP data using a kernel smoothing technique to help detect the pattern extrema. The statistical Tests used are Kolmogonov-Smirnov and a particular form of Chi-squared test using return quantiles; however, even these tests within the same paper often disagree as to which patterns display significant abnormal returns and which do not. Though the findings are mixed the use of standardised language and simple rules clarifies the approach; and the kernel smoothing methodology is sensible to detect pattern extrema. Both of these ideas I reuse after significant modification of the methodology in order to correct issues and produce more transparent results.

Several papers follow from Lo et al. (2000), usually using the same language constructs and similar style of rules to understand the patterns, but using other detection methods and statistical testing techniques. These include the use of fuzzy logic, neural networks, genetic algorithms and various hybrid approaches.

Ahlawat (2015) uses an interesting probabilistic neural network idea applying techniques used for developing character recognition software, where these algorithms are trained to recognise chart patterns. I considered using similar techniques but the problem with training neural networks is that the pattern selection reasoning is opaque we do not know how or why the pattern was selected and additionally cannot fine tune it. While Ahlawat (2015) does not find significant abnormal returns, the method used to statistically test returns is not clear, so no conclusion can be drawn.

Escobar et al. (2013) uses fuzzy logic and decision trees to identify patterns, finding significant abnormal pattern returns for some cases where this depends on specific rules applied. In the paper a CAR (Cumulative Abnormal Return) based F-test and Kruskal-Wallis test are used, which do appear to have merit in determining the period of significant abnormal returns. Yu et al. (2011) uses a series of simple filter rules based on relative percentage price movements which, while easily understood, are inconclusive as to which filtering rules produce the correct set of patterns. This produces a large set of test results for various rules, some of which show patterns having significantly abnormal returns, and some of which do not. From these two papers it seems clear that an algorithmic approach is easily applicable to detection of patterns. It seems odd however, that the authors used fairly common algorithmic approaches rather than writing bespoke algorithms that can be more directly tailored to exact detection. In response I wrote a bespoke detection algorithm in combination with using the kernel smoothing approach to identify price extremum as shown in the method chapter. 
Unfortunately from this pattern based research it is not clear from the statistical testing procedures displayed in these papers what the best approach is to take; and often the testing logic is not clear and so no conclusions can be reliably drawn. None of the papers take account of transaction costs and short sale restrictions. Often the papers disagree as to which forms of the patterns have significant abnormal returns and which do not. All studies take a mechanical approach to identifying verbatim chartist patterns and attempt to test standard daily returns with no trade execution considerations of how real traders would place positions. The methodology and p-value testing based procedures used are not convincing and yet a brief review of other finance papers shows that the methods used seem reasonably standard.

It appears little or even no effort may have been put into digging more deeply into what traders are identifying and how they are seeking to profit. From my experience working on a trading desk I question whether the authors have an understanding of how the strategies are used for trading and how these are to be executed for profit. This seems surprising since this information is widely available, for example see Brant (2011) which shows the reasoning used with a well audited track record.

I would suggest that recording daily returns from end of day close to close, after the pattern event occurs, does not represent actual returns well; rather it gives an indication of how price develops after the pattern ignoring real trade position execution considerations, and this is unrealistic. An actual trading return consists of an entry price, with a risk controlled approach and an exit price with round trip transaction costs subtracted. These are considerations before any trade is placed and are well known and often reasonably standard. I consider Lo et al. (2000) to be of substantial quality and other research to have such a numerous array of problems that it is better to focus on Lo's work which provides reasonably well grounded research methods and several usable creative ideas. More detail is given in the Method Chapter 3.

\subsection{Research Methods}

Despite there being some interesting results, the knowledge that can be derived from much of the literature on pattern trading seems limited, largely because the research methods are not robust. This is disappointing and prompts a review of research methods to determine how best to proceed. While such problems of methodology as exemplified by the reproducibility problem in social sciences are well known and attempts have been made to combat this, such concerns seem not to have been addressed in the finance literature to the same extent. The pattern based trading literature has used statistical testing techniques that would not be acceptable in many other disciplines.

The sparse finance research available in this area includes work by Harvey et al. (2016) 
which concludes 'most claimed research findings in financial economics are likely false' and Bailey et al. (2014) which show how easily over-fitting can misreport results. In finance and economics I have repeatedly observed $\mathrm{PhD}$ students persistently searching for significant effects in the same data set, then writing papers using 'apparent significance' that has clearly involved data mining. The pressure to publish forces unseen ethical problems of a potentially large but immeasurable size. From the trading industry which demands profitable results Carver (2015) poignantly points out that even using apparently perfect methods it is often impossible to not data-snoop by having prior tacit intuitive knowledge gained from elsewhere. For example based on Lo et al. (2000) I could easily make simple data-snooped improvements using the same dataset and report apparently more significant but spurious findings. While it seems unwise to be overly pessimistic, it is prudent to take precautions and other fields have a variety of useful best practices to consider.

The breadth of methods used in other fields is well summarised by literature from psychology, biology, medicine and physics where there are a variety of suggestions often hinging on alternatives to p values. Schwab et al. (2011) summarises these well from the wider literature as follows:

1. tailor assessment to research context;

2. report uncertainty associated with effect size;

3. explain and illustrate assessment indicators;

4. compare new data with naive models rather than null hypotheses;

5. to support generalization and reproducibility, frame hypotheses within very simple models;

6. use robust statistics to make estimates, especially robust regression.

To correct the pattern trading research approach I follow all of these recommendations tailored specifically to Triangles. In particular I make use of a similar method devised by Connor and Simberloff (1986) to evaluate non-experimental evidence regarding Galapagos Island finch evolution where a Naive model is used as a comparison. I make use of effect size based statistics and recommendations as per Cumming (2012) to comply with new statistical research guidelines being adopted to combat reproducibility problems. To avoid an inordinately wide description of these corrections I comment further in the Method chapter.

\subsection{Summary}

- The Efficient Markets Hypothesis is generally used in finance as the null hypothesis for evaluating trading returns, where returns are often modelled using stochastic processes. In effect the null hypothesis taken from this to test trading methods with the view that price cannot be predicted; and so conditioning on trading events will 
produce no abnormal returns.

- There are a variety of stylised facts for returns, and there is some contention about common EMH theoretical return arguments based around Behavioural Finance. The Adaptive Markets approach seems sensible as a means to justify a fractional market inefficiency.

- Overall the use of models assuming efficient markets and periodic returns is overly prescriptive and some thought needs to be given to create both more realistic returns and a meaningful comparative model, which motivates the use of naive models.

- Pattern based trading literature shows some significant abnormal returns, particularly by Lo et al. (2000); however later literature disputes this. A core problem with this trading literature is that the research methods used are not particularly robust.

- For pattern trading it seems better to consider trading from a traders point of view, applying best practices from the trading industry and stronger research techniques designed to correct the issues found in pattern based trading literature. 


\section{Chapter 3}

\section{Method}

This chapter describes the method of results capture based on price level Triangle trading. I focus on interpreting Triangle trades as real short term momentum traders do, where I consider taking positions to capture profit based on short term leverage. To this end, the Triangle event is defined and arguments are made as to how returns are captured using price levels and risk management. Then a trading model called a Naive trade is defined with which to compare the value of Triangle trade timing based returns. Overall this directs the research results towards effect size based statistics, such that differences in returns can be evaluated more clearly. This is done to concur with more improved research best practices. Once events are defined, algorithms are described to capture Triangle and Naive model trade events. To clarify the information value of Triangles results, these are captured in terms of raw price change, and trade holding period. The idea is to then capture a measure of momentum that is closely aligned to a short term trader's perspective, such that realistic measurements can be statistically modelled. Finally, at a conceptual level I introduce the modelling approaches, which I detail in later chapters.

The finance literature often uses standardised periodic returns to evaluate market efficiency, often without considering trade entry or exit timing; and while this is not always the case, this has certainly been true with the pattern trading literature reviewed in this study. In contrast, and in the knowledge of how traders operate in practice, I take into account how real traders take positions in the market, and use raw price levels to evaluate returns. Their methods are market timing tools based on price levels, which are ideally used to take short term profits. Brant (2011) shows a detailed and audited account in a diary format of real trades taken using these ideas which I use as a reference for pattern technical trading. While there are numerous other references, the advantage of using Brant's ideas are that these are fairly standard, he has a long well audited trading record of consistent abnormal profits, and that he describes his trading methodology is considerable detail. 
Drawing on this knowledge I construct an informed framework to interpret Triangle events; and construct a return interpretation based on price levels and first passage times. In this context, the goal of the interpretation is to identify the short term momentum effect to evaluate the possibility of abnormal returns. In doing so, a set of returns in terms of changes in price to fixed levels and first passage times are captured to uncover the effect size.

Triangle capture is done using risk/reward based position sizing with algorithms written to capture Triangle events and return data by simulating level based trading. While machine learning was an attractive means to this end, given some of the difficulty with opaque methods this was avoided, such that the rules of data capture are transparent. It was also more desirable to capture trades exactly using a defined mathematical formula, but this proved elusive.

\subsection{Triangle Trades}

The Triangle definitions in this section are based on price levels, where the Triangle is defined in terms of relative extrema, and leverage is used to capture short term momentum. Returns are calibrated to the height of the Triangle, such that trades are designed to have comparable returns. Focusing solely on Triangles facilitates specific well developed definitions and evaluation, which would be more difficult if more general trading methods and patterns were allowed.

Two main techniques used to trade Triangles are level based and trend continuation; the latter is similar to a buy and hold strategy. While there are a plethora of ideas as to how to trade such patterns, the level based method is particularly common; also this method is quite directly measurable and hence more feasible to capture than other more subjective methods. Adding trend interpretation and variable holding period is additional work which is more ambitious and better left till later research. Level based execution requires a specific set of opinionated choices about the price and time at which trades are executed, and these are relatively easily constructed. 'Opinionated' in this context refers to industry software engineering based approaches to creating a repeatable style of product development: see Bedell (2006) for a description.

My definition of a Triangle is specific; while various definitions could be used I have restricted Triangles in this thesis to be of a smooth type which appears in the shape of a dampened converging wave, where I take into account exactly four relative extrema, as previously shown in 1.1 and 1.3. This strategy selects a very clear type which can show the effect size easily by defining entry and exit points based on levels; and also being simple enough such that a large sample size can be obtained. While other types with more extrema and other features could have been captured, focusing on a very simple case makes detection and evaluation more transparent. 
Hence in this study Triangles consist of four types defined by whether they are a top or bottom as seen in prior work done by Lo, and whether they break up or down as defined by how traders take positions based on support and resistance levels. These are individually described in the following subsections and shown in Figures 3.1 to 3.4 as straight lines from each subsequent significant point. The variables and geometry defined from these are used later to model short term momentum. Section 2.1.3 provides insight into how these Triangles are traded and how this is formulated in an algorithm.

The four types of Triangles are described in terms of time and price based points, which is illustrated in Figures 3.1 to 3.4:

- A: point above or below the Triangle that price arrives from,

- $E_{1}$ to $E_{4}$ : Extrema representing the Triangle shape,

- $B$ : point at which price breaks out of the Triangle shape and simulated traders enter their position,

- $C$ : point at which simulated traders close their position (either $C^{+}$at $+h$ for a win or $C^{-}$at $-h$ for a loss).

In this way the Triangle measurements are defined in two parts, consisting of segments $A$ to $B$ and $B$ to $C$. Segment $A B$ defines the triangular shape and segment $B C$ captures the return. Within $A B$ each Triangle is defined by four extrema $E_{1}$ to $E_{4}$, with the height defined by $h=\frac{\left|E_{1}-E_{2}\right|}{2}$. Measurements of $h$ and volatility $\sigma$ are considered defined at point $B$ and then fixed until point $C$. The trading results are then defined from the time taken for the trade to move from point $B$ to $C$ by one unit $h$ in time $\tau$. To summarise, results for each Triangle consist of:

- $h$ representing the height of the Triangle as $h=\frac{\left|E_{1}-E_{2}\right|}{2}$,

- $\sigma$ as defined by volatility for that security,

- $\tau$ for time taken for the trading position to open and close over $B C$, resulting in either a win at time $\tau^{+}$or a loss to time $\tau^{-}$

- $r$ for the Bet Return as further defined in Section 2.1.3 as a proportion of $h$ defined as $r=\frac{C-B}{h}$ for long trades and $r=\frac{B-C}{h}$ for short trades (generally $r=1$ for wins and $r=-1$ for losses, but in certain cases the series is cut short so can be $r \in[1,-1]$ ).

The four types of Triangle are described using a similar pattern based trading language as seen in Lo et al. (2000) with an initial lock-in arrival point $A$, and using OHLC prices to measure extrema $E_{1}$ to $E_{4}$. This is followed by the addition of level based trading execution from $B$ to $C$ defined by change in price $h$. Triangles are identified by a noun verb phrase 'type' followed by 'direction'. There are two types, 'TTop' for Triangle Top and 'TBot' for Triangle Bottom; and two directions, ' $\mathrm{Up}$ ' for a price move upwards and 'Down' for a price move downwards. These are described without price smoothing and spacing concerns which are later addressed by the algorithm in Section 3.2.4. In the following definitions the points $A, E_{1}, E_{2}, E_{3}, E_{4}, B, C$ occur sequentially in time to form the significant defining Triangle points. Over segment $B C$, green lines are placed on the 
figures to indicate profitable trades ending at $C^{+}$, red lines to indicate losses at $C^{-}$. For TTop $E_{1}$ is defined to be a maximum extremum over segment $A E_{4}$; for TBot $E_{1}$ is defined to be a minimum over segment $A E_{4}$.

\subsubsection{TTop Up (Triangle Top Break Up)}

Price rises from below the Triangle formation, starting at $A$ moving to maximum $E_{1}$, forming the Triangle of $E_{1}$ to $E_{4}$, and breaks out of the pattern by starting at $E_{4}$ and going to level $B$ without going below $E_{4}$. The trader then buys at the break point $B$, intending to sell either where price rises by $h$ to close point $C^{+}$for a win or where price falls by $h$ to close point $C^{-}$for a loss. This can be seen in Figure 3.1 and (3.1).

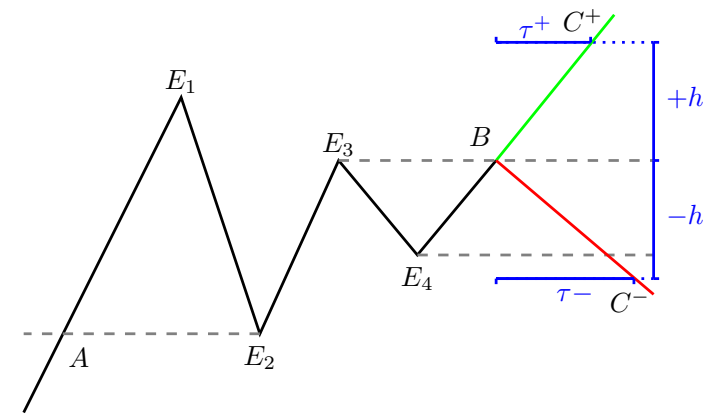

TTop Up $=\left\{\begin{array}{l}\exists A<E_{2}: A<P_{t}<E_{1}, t_{A}<t<t_{E_{1}} \\ E_{1} \text { is a maximum } \\ E_{1}>E_{3}>E_{4}>E_{2} \\ \exists B \geq E_{3}: E_{3}<P_{t}<B, t_{E_{4}}<t<t_{B}\end{array}\right.$

Figure 3.1: TTop Up

\subsubsection{TTop Down (Triangle Top Break Down)}

Price rises from below the Triangle formation, starting at $A$ moving to maximum $E_{1}$, forming the Triangle of $E_{1}$ to $E_{4}$, and breaks out of the pattern by starting at $E_{4}$, moving toward the centre of $E_{4}-E_{3}$ and going to level $B$, without going above $E_{3}$. The trader then sells short at the break point $B$ intending to buy either where price rises by $h$ to close point $C^{-}$for a loss, or where price falls by $h$ to close point $C^{+}$for a win. This can be seen in Figure 3.2 and (3.2).

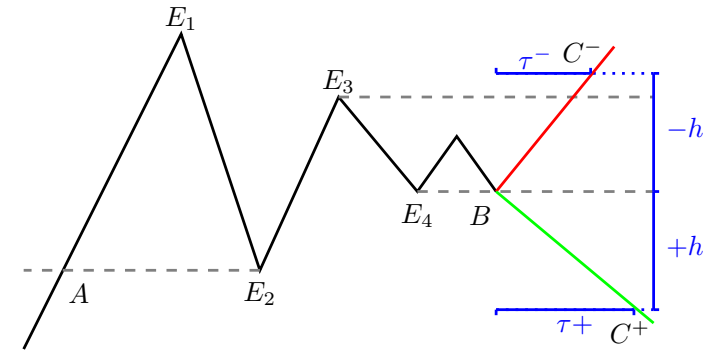

Figure 3.2: TTop Down
TTop Down $=\left\{\begin{array}{l}\exists A<E_{2}: A<P_{t}<E_{1}, t_{A}<t<t_{E_{1}} \\ E_{1} \text { is a maximum } \\ E_{1}>E_{3}>E_{4}>E_{2} \\ \exists B \leq E_{4}: B<P_{t}<E_{3}, t_{E_{4}}<t<t_{B}\end{array}\right.$

\subsubsection{TBot Up (Triangle Bottom Break Up)}

Price falls from above the Triangle formation, starting at $A$ moving to minimum $E_{1}$, forming the Triangle of $E_{1}$ to $E_{4}$ and then breaks out of the Triangle pattern by starting at 
$E_{4}$, moving down toward the centre of $E_{4}-E_{3}$ and going up to level $B$, without going below $E_{3}$. The trader then buys at the break point $B$ intending to sell either where price rises by $h$ to close point $C^{+}$for a win or where price falls $h$ to close point $C^{-}$for a loss. This can be seen in Figure 3.3 and (3.3).

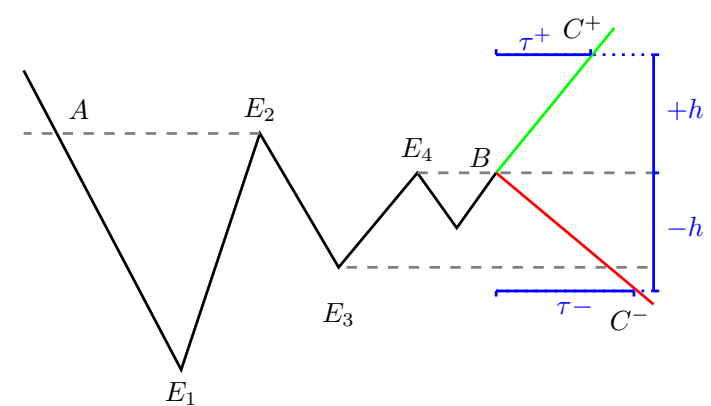

Figure 3.3: TBot Up
TBot Up $=\left\{\begin{array}{l}\exists A>E_{2}: A>P_{t}>E_{1}, t_{A}<t<t_{E_{1}} \\ E_{1} \text { is a minimum } \\ E_{1}<E_{3}<E_{4}<E_{2} \\ \exists B \geq E_{4}: B<P_{t}<E_{3}, t_{E_{4}}<t<t_{B}\end{array}\right.$

\subsubsection{TBot Down (Triangle Bottom Break Down)}

Price falls from above the Triangle formation, starting at $A$ moving to minimum $E_{1}$, forming the Triangle of $E_{1}$ to $E_{4}$ and then breaks out of the Triangle pattern by starting at $E_{4}$, and going down to level $B$, without going above $E_{4}$. The trader then sells at the break point $B$ intending to buy either where price rises by $h$ to close point $C^{-}$for a loss or where price falls $h$ to close point $C^{+}$for a win. This can be seen in Figure 3.4 and (3.4).

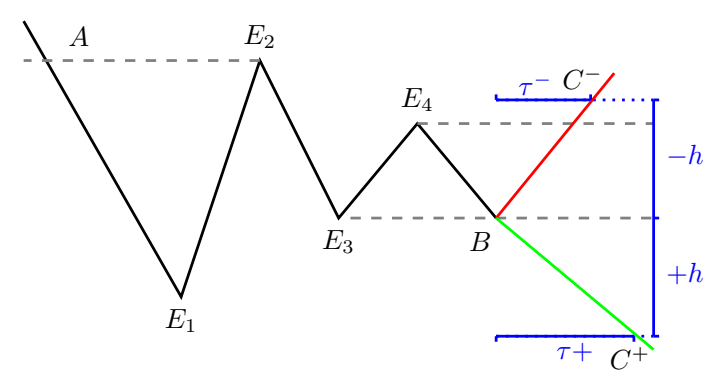

Figure 3.4: TBot Down
TBot Down $=\left\{\begin{array}{l}\exists A>E_{2}: A>P_{t}>E_{1}, t_{A}<t<t_{E_{1}} \\ E_{1} \text { is a minimum } \\ E_{1}<E_{3}<E_{4}<E_{2} \\ \exists B \leq E_{3}: B<P_{t}<E_{4}, t_{E_{4}}<t<t_{B}\end{array}\right.$

\subsubsection{Returns}

Triangle returns are evaluated in terms of entry and exit points based on changes to fixed price levels. The trader's perspective is that once price breaks a significant level (in this case one of the last two extrema $E_{3}$ or $E_{4}$ ), price is expected to move quickly outside the Triangle range. Then price at some point is believed to return to a more unpredictable state, at which point or before this the trader exits the position. While trade entries are usually taken where the price breaks through a significant price level, in this case at point $B$, the exit policy is often less clear. So to standardise, I enter the trade at the first broken extremum and exit at half the total height of the Triangle, measured as $h$ for a win or loss. Beyond the change in price $h$ it seems more likely that the Triangle will have less effect. 
This standard ideally captures any informational value of the Triangle, by capturing at the very least the start of a tradable price move after the relevant Triangle extremum level has been broken. This exit level at change in price $h$ is not exactly tailored to each Triangle as is the entry price, but this enables the creation of a standard measurement based on leverage of trade returns across all Triangles.

Traders seek to enter short term positions using leverage, moving capital between positions to quickly maximise gains and reduce draw down by spreading risk across multiple trades. This spread of risk is often done by allocating trade equity using potential risk/reward measures across multiple positions through time, rather than managing risk using portfolio balancing as is done with buy and hold strategies. With Triangles the traders seek to gain advantage through timing of entry and exit points, by predicting short term momentum over that period. Raw risk is measured as the maximum amount able to be lost for that trade, and the trade is leveraged through either debt or excess cash reserves - though I do not model this to retain simplicity.

To model this proportionate risk, $\mathcal{R}$ is used as defined in Tharp (2008) to model risk/reward, which creates a simple framework to standardise position sizing using leverage. Using this method a $\mathcal{R}$ value is assigned to the trade as

$$
\begin{array}{rlr}
\mathcal{R} & =\frac{\text { expected win amount }}{\text { expected loss amount }} & \text { standard } \mathcal{R} \\
& =\frac{n\left|P_{\text {win }}-P_{i}\right|}{n\left|P_{\text {loss }}-P_{i}\right|}=\frac{\left|P_{\text {win }}-P_{i}\right|}{\left|P_{\text {loss }}-P_{i}\right|}=\frac{|h|}{|h|}=1 & \text { normalised to } 1,
\end{array}
$$

where $\mathrm{n}$ is the number of shares and $P$ in price. This is done as a way to measure the risk/reward and appropriately allocate trade position size. For this study I standardise $\mathcal{R}=1$ by making the win and loss values equal to $h$, where transaction costs are added after the $\mathcal{R}$ calculation. So one unit of $\mathcal{R}$ is then measured in $h$. This is done so that every trade is leveraged to produce the same unit of standard risk/reward, such that profit or loss from each trade is denoted $r= \pm 1$.

This produces a leveraged return and expected profit as given by

$$
\begin{array}{r}
\qquad r_{l}=\frac{p \mathcal{R}}{\tau}=\frac{p}{\tau} \\
\text { Expected Profit }=E(X)=p h n=p k,
\end{array}
$$

where $p$ is the probability of winning, $r_{l}$ is level based return and $k=h n$ which is the amount risked on trade close.

This in a sense nearly inverts the problem from a periodic return defined on an interval of time and variable price, to a return based on on a standardised level based on $h$ using $\mathcal{R}=1$ to produce an expected profit of $p k$ and a variable close time $\tau$. For $k=1$ results 
can be formulated as a Bernoulli random variable with expectation

$$
\mathbb{E}(X)=\sum_{\{1,-1\}} x f(x)=p-1(1-p)=2 p-1
$$

and variance

$$
\begin{aligned}
\mathbb{E}\left(X^{2}\right) & =\sum_{\{1,-1\}} x^{2} f(x)=p-(-1)^{2}(1-p)=1 \\
\operatorname{Var}(X) & =\mathbb{E}\left[(X-\mathbb{E}(X))^{2}\right]=1-(2 p-1)^{2}=4 p(1-p) .
\end{aligned}
$$

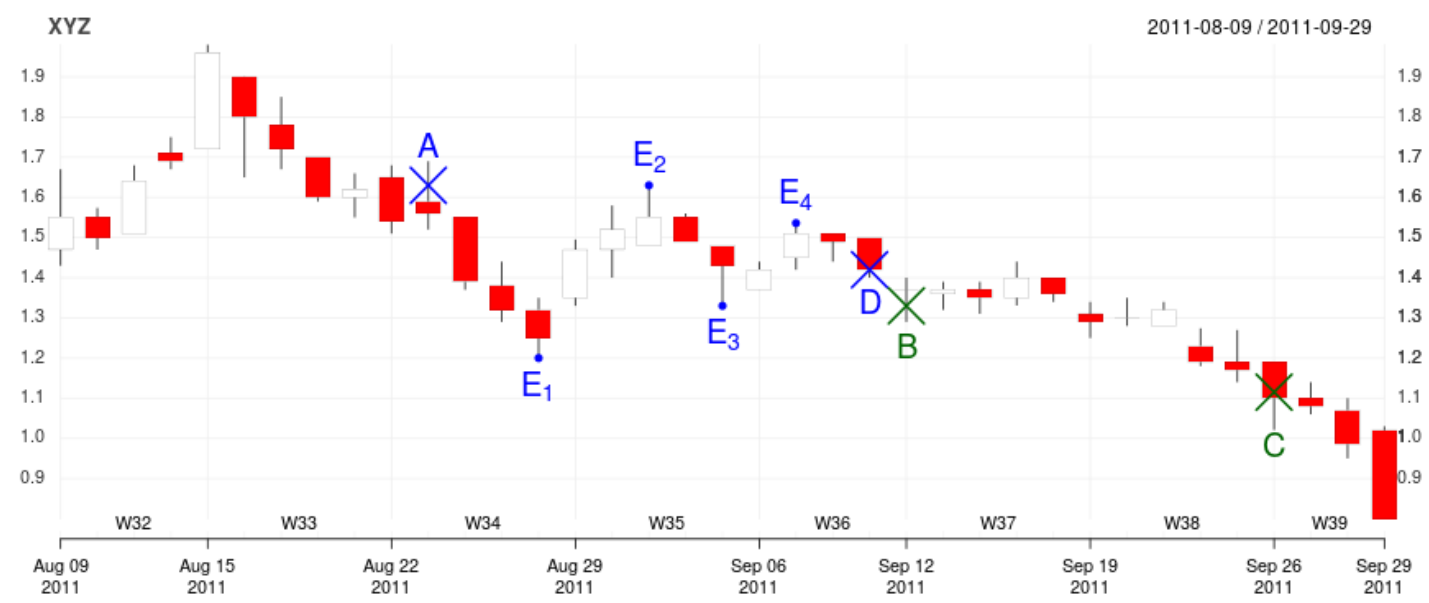

Figure 3.5: XYZ Equity TBot Down Trade Example

This is best illustrated with a practical example: A trader recognises a TTop Down Triangle with stock XYZ as seen in Figure 3.1.5 with $h=0.215$, which is the expected change in price. The Trader wishes to risk $\$ 10,000$ of capital by selling short at point $B$ where the price of $X Y Z$ is 1.35 . He does so with an expectation that he has a $60 \%$ chance of success, so on balance thinks the trade is worth $[2 p-1] k$ or $(2 \times .6-1) \times 10000=\$ 2000$. So he must sell $\frac{10000 \times P_{B}}{h}$ or $\frac{10000 \times 1.35}{0.215}=\$ 62790.7$ worth of stock or roughly short 46512 shares, in order to setup the proportionate amount of defined risk to gain or lose $\$ 10,000$ on trade close. The price moves to point $C^{+}$where the position is then bought for a win of $\$ 10,000$ less transaction costs in approximately 11 days. If the trader had lost at point $C^{-}$(not shown) he would have then lost $\$ 10,000$.

The Trader will have many such positions and will borrow to support a portfolio of such trades with a set amount of overall risk as defined cumulatively by $\mathcal{R}$ calculations. Realistically this would involve more complex considerations of transaction costs, trade correlation and slippage across the trade book and be subject to corporation risk management rules, not to mention using various values of $\mathcal{R} \neq 1$; however my 
approach provides a useful simplified comparative basis.

Two measurement types concerning these are information content and profitability. The first, information content, emphasises testing that the distribution of returns, usually without transaction costs, are significantly different from what would be expected under the null hypothesis that markets are efficient. The second focuses on the profitability of a trading strategy. Focusing purely on profitability can result in ignoring the underlying cause of excess return. Excess return could be due to the behaviour of investors being willing to accept higher returns at the cost of more risk or volatility, so profitability may not indicate significant deviation from efficient markets in that returns are risk adjusted.

Lo et al. (2000) uses the informational approach to establish whether pattern based technical analysis is informative by comparing the returns of stocks conditioned on patterns with the Unconditional case. Lo finds that there is a difference with some patterns, in particular finding some Triangle patterns do have a different distribution of returns, including some Triangular tops; but he does not investigate further. However because of the lack of effect size based comparison, where return amounts are not reported clearly, it is not clear what the difference is between EMH based returns and Triangle returns.

By standardising using $\mathcal{R}=1$ and simulating real discretionary traders I create a measurement which can be used to evaluate both the information content in terms of Wins of $r=1$ and Losses of $r=-1$, and the profitability by subtracting transaction costs. I refer to $\mathbb{E}(X)=r_{b}$ as Bet Return where there is no time component to differentiate from time based returns. Through leverage this approach simplifies later analysis of price changes by normalising to a Bernoulli random variable, such that the amount gained from each trade is consistently measured. Return and variability can now be measured purely in terms of probability, with the later addition of time. Hence raw returns are measured in change in $h$ and $\tau$ standardised to amounts gained of $r= \pm 1$ with the aim to create a clear and standardised measurement of effect as distinct from periodic returns, as is traditionally done in finance. Thus simplified, what remains to be understood is the time $\tau$ expected to close the trade.

\subsection{Triangle Detection}

As in Lo et al. (2000) I use kernel smoothing; however in addition I make use of algorithmic methods to develop a more accurate Triangle detection method for wider time windows across significantly larger datasets. This is done by using a simple map-reduce method, to make use of multi-core computational power, using a forward rolling window scan. The definitions of Triangles given in Section 1.1 provide the framework for determining the geometry of points for each Triangle type; these are used with a recursive search of kernel bandwidths to determine valid extrema spacing and 
smoothness. The end result is a computational method which can scan any large OHLC time series in a timely manner on a grid network to produce a set of unique Triangles. Triangles are captured in an object orientated form which can be manipulated for later analysis and are stored in a hybrid hashmap/list.

The core starting difference between prior literature and this approach is that the Triangle pattern is detected based on trading events defined in terms of price levels rather than periodic returns. I have used an exact walk back algorithm to detect alternating price extrema which are compressing toward a focal point. The approach consists of evaluating the time series sequentially backward from the current time for geometric triangular shapes, which may not be adequately spaced or smooth; these shapes are then validated using the recursive kernel smoothing approach. This is done via a binary search over kernel bandwidths to find the most appropriately smoothed extrema. If a well formed Triangle is found the algorithm walks forward to determine the trade holding time and profit/loss at the appropriate stopping time. The walk forward is done sequentially from point $B$ to $C$ to determine each of the pattern results. I note that point $B$ is the crossing time and level as described, and that the walk backward requires a separation of one period from point $B$ to avoid data snooping within that period. I describe the process in pseudo code as a series of function calls nested within detectTriangle() in algorithm 3.1.

The data series used are large; for US Equities for instance data consists of more than 27000 time series of OHLC prices. To deal with this the processing has been distributed across a grid computing environment using a simple map-reduce method. Each time series or overlapping section of long time series are submitted (mapped) as jobs to the grid, and Triangles are detected and saved using identification by time series detection time, type and security. Once all processing has been done for all time series, duplicates are removed, overlapping Triangles of the same type are removed, and various calculations are carried out. Each grid job runs a separate detection algorithm instance run from bash using $\mathrm{R}$ based on Linux.

\subsubsection{Improvements on Literature}

Lo et al. (2000) uses kernel smoothing over a fixed period of 38 days for US stocks to detect extrema, where extrema must occur within the first 35 day period of this window. This 38 day window scan is rolled forward through the time series to detect patterns, and where patterns are found, a one day gross return is calculated after the pattern (using a day of price lag to separate detection from returns to avoid single period data snooping). To detect these patterns Lo uses his pattern trading language to define extrema geometry as seen in (3.9) for Triangles. Spacing and smoothness are detected using kernel smoothing with a fixed bandwidth of $0.3 l$ where $l$ minimises $C V(l)$ given by 


$$
\begin{aligned}
C V(l) & =\frac{1}{T} \sum_{t=1}^{T}\left(P_{t}-\hat{m}_{l, t}\right)^{2} \\
\hat{m}_{l, t} & \equiv \frac{1}{T} \sum_{\tau \neq t}^{T} \omega_{\tau, l} T_{\tau} .
\end{aligned}
$$

This is a less than fully successful attempt to produce a kernel bandwidth appropriate to each Triangle. The results of this algorithm are validated by Technical Analyst specialists on that same US equity data. It appears that a cross-validation based bandwidth is chosen by looking ahead using human analysts to validate the pattern shapes until the best bandwidth is chosen. This has likely resulted in the use of in-sample data to check results which forms a not so obvious look ahead bias. Also using a semi-fixed bandwidth approach does not seem optimal as the smoothing will work better in some instances and much worse in others, a point Lo admits. It would seem better to select bandwidth on a case by case basis, which is probably the idea behind using the cross validation method. When I tried this on GBPUSD data the results were very rough, since the detected Triangles often were not well formed, though this conclusion is rather subjective.

Also Lo does not consider overlapping detection of Triangles, which occurs frequently, such that Triangles often share the same set of extrema, or have similar extrema. This occurs where rolling the window forwards often detects the same or similar Triangles, and neglecting this seems a surprising oversight in the literature, since this becomes immediately obvious when implementing these techniques. Lo's method was applied using closing prices, but typically OHLC prices are almost always used with pattern based trading, where the high and low prices are generally included to help define the significant levels which are traded against. Once patterns have been found, data is normalised using return drift and volatility from the entire data-series, which is direct data snooping. Overall, this is a good first exploratory effort, but can be greatly improved.

For data Lo uses the Centre for Research in Securities Prices (CRSP) NYSE/AMEX and NASDAQ stocks from 1962 to 1996. The total time period is divided into seven 5 year blocks and a quintile by market capitalisation to select 50 stocks in each sub-period, all of which must have $75 \%$ of their prices available within that 5 year sub period. This involves look ahead bias and survivorship bias, since it dictates a knowledge of prices ahead of time and its subdivision into quintiles.

To fix the issues with Lo's approach I do the following for US Equities:

1. use the entire US equity dataset rather than selecting 50 stocks for each historical section quintile;

2. selection based on $75 \%$ of prices being available within the scan walk back period 
rather than looking ahead in time (see Appendix B for more detail on the management of data);

3. change the nature of the Triangle by reducing the number of extrema from 5 to 4 in order to increase the sample size, to maximise the power of statistical testing while retaining the triangular shape;

4. include an initial 'lock in' point $A$, signifying the Triangle arrival which confirms that $E_{1}$ is an extremum within a defined range and signifies the start of the triangular shape (this more accurately forms a Triangle as a break out strategy);

5. use the break point $B$ and close point $C$ to define timing such that returns more realistically represent actual returns;

6. use trial GBPUSD data as opposed to the main data of interest, viz. equities, to develop the approach - to avoid look ahead or data snooping problems;

7. use a wider period of 200 days for walk back to find Triangles;

8. use a widening backward search algorithm on raw price to find alternating extrema that form the Triangle type as per the definition given in this chapter;

9. on a case by case basis set the kernel bandwidth to best reflect the extrema found using a binary search such that the smoothness used is relative to the size of the Triangle;

10. normalise each Triangle individually based on the previous 100 days of volatility before point $B$ to avoid data snooping;

11. remove all duplicates using hashing based on security symbol, Triangle type and extrema time to uniquely identify each Triangle;

12. avoid the overlap problem by requiring all Triangles of the same type to not have any of the same extrema by declaring the first pattern with the same extrema which breaks, to be the one counted, where others with the same extrema are removed (filtered iteratively forward in time);

13. use OHLC prices to reflect extreme highs and lows that discretionary traders normally take into account.

For the trials using ES futures and GBPUSD data I modify this approach by using a 500 minute look back window, with volatility measured over the entire time series, whereas for US equities I measure volatility separately for every Triangle. This is done since for exploratory purposes it is less important to be exact. I also trial a range of different methods using the ES futures and GBPUSD data which help inform the final approach without compromising the integrity of US equity data usage.

\subsubsection{Grid Map-Reduce}

An unpublished R package called 'triag' was written to handle Triangle detection and manage data manipulation. This includes producing various graphics and tabular data, some of which are generated and placed in this thesis. Using the triag package Triangle scans are run on Victoria University's Sun Grid Engine which distributes jobs across 
hundreds of client Linux machines within the School of Engineering and School of Mathematics and Statistics. The algorithm selects high quality Triangular patterns but is a relatively unoptimised, exhaustive search across a large dataset. To complete this work the processing time is large but by distributing the load this can be processed within two weeks (including Naive trade processing).

The search task is split using a map-reduce strategy to distribute jobs on the grid and later aggregate results. For long single time series (GBPUSD and ES) the time series is split into 50000 minute blocks and the tail 1000 OHLC prices are concatenated onto the head of the previous block of prices to produce blocks of 51000 minutes including the overlap, followed by the odd numbered tail block of prices which does not have the overlap. These are distributed as jobs to the Sun Grid Engine where the R package scans for triangles. Once the grid jobs have completed, all Triangles are aggregated and duplicates are discarded. For the stock time series these are split for large time series but otherwise are scanned as they are. Overlaps of the same type of Triangle are dealt with by accepting the Triangle that breaks first and rejecting other overlaps iteratively forward in time. All four types of Triangle are detected and saved using this method in one scan pass over the grid. This reduces the processing time taken for scanning to days, where this would potentially have taken several months.

\subsubsection{Rolling Window Scan}

For US equities a backward window scan size of $w=200$ days is used, considerably larger than the 35 days Lo uses. Once a pattern is found Lo calculates the forward one day gross return; however, my method is to walk forward until the $\pm h$ level is hit or the series ends, and so the forward window is both often longer and of variable length. I consider a year to have roughly 250 days, and so 200 days captures a significant proportion of the year; and the majority of results is ideally caught within the remaining 50 days to roughly represent a year under consideration. Traders are interested in short term trading and so the window is limited to 200 days for equities to exclude longer term Triangles of less interest; in fact most Triangles are probably considerably shorter but it seems useful to capture a relativity complete set of results without rerunning the data and succumbing to data snooping.

For US equities, the walk backward algorithm scans 200 days backward to find potential triangular patterns which are checked for smoothness. When a pattern is found, the detection point $D$ is marked. From this point the algorithm walks forward to determine where it breaks at point $B$, which must be at least 1 day later. The period taken to reach $B$ from $D$ labelled is $b$ and the walk back window of 200 is labelled $w$. Over segment $B C$ the amount won or lost and time taken $\tau$ is then determined. So the total window size consists of time series segments $(D-200) D, D B$ and $B C$ which results in a total window size of $w+b+\Delta \tau \geq 201$. Triangles are uniquely identified by their type, security and break time where no extrema overlaps of the same security and Triangle type are 
allowed. The 200 period window scan effectively scans step by step, from the start of each time series plus 200 to the end of each time series minus 1 (to allow for the break out).

This is done such that Triangles are captured in time blocks generally of 10 years (except for the first), where Triangles belong to their time block as determined by their break date at point $B$, allowing the forward window to overlap into the next time block. This means each time block's results are representative of Triangles that break within that historical period, where some longer time frame returns overlap.

During the scanning multiple duplicates and overlaps are found. Using hashing to uniquely identify the each Triangle, only the first duplicate is stored and the others are discarded. To solve the overlap problem, only the first Triangle of its specific type (as detailed in 1.1) that breaks first is kept, while all others of the same type with any of the same extrema are removed. This is done as a simple way to achieve a degree of Triangle independence and avoid potential issues of double counting.

\subsubsection{Detection Algorithm}

Algorithm 3.1 detectTriangle(): detection algorithm root method starting point which finds the attempts to find a Triangle. This is called on each step forward in the scan through the time series.

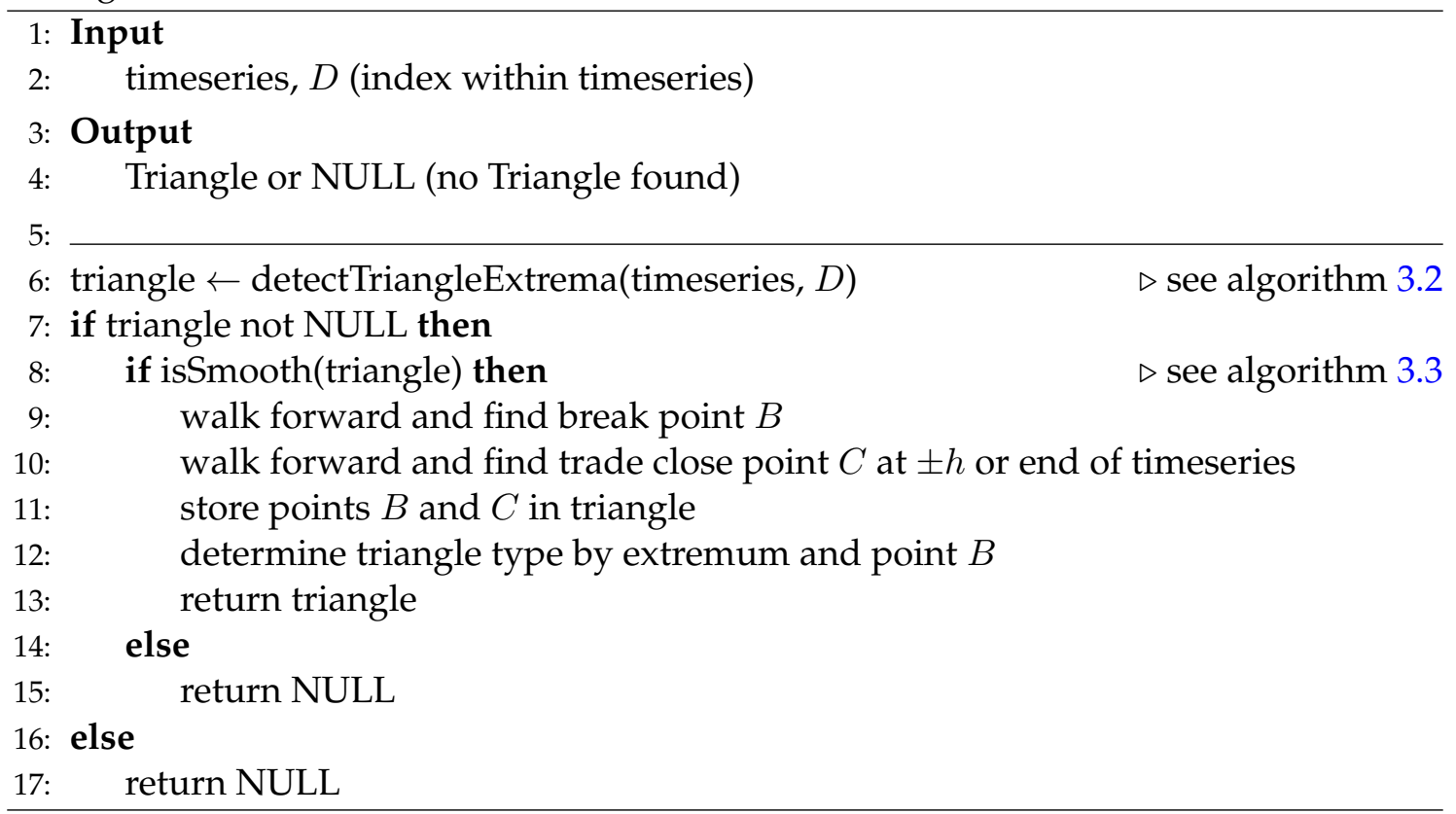

The detection code revolves around scanning for Triangle objects and determining the return results, since in this study Triangles are defined not only in terms of their smooth shape, but also their definition depends on how price breaks outside of the price range towards $\pm h$. To achieve this I use a mixture of objection orientation and recursive functional programming, which keeps the code terse and happens to provide a level of abstraction that can be mapped reasonably well to pseudo code, without using more 


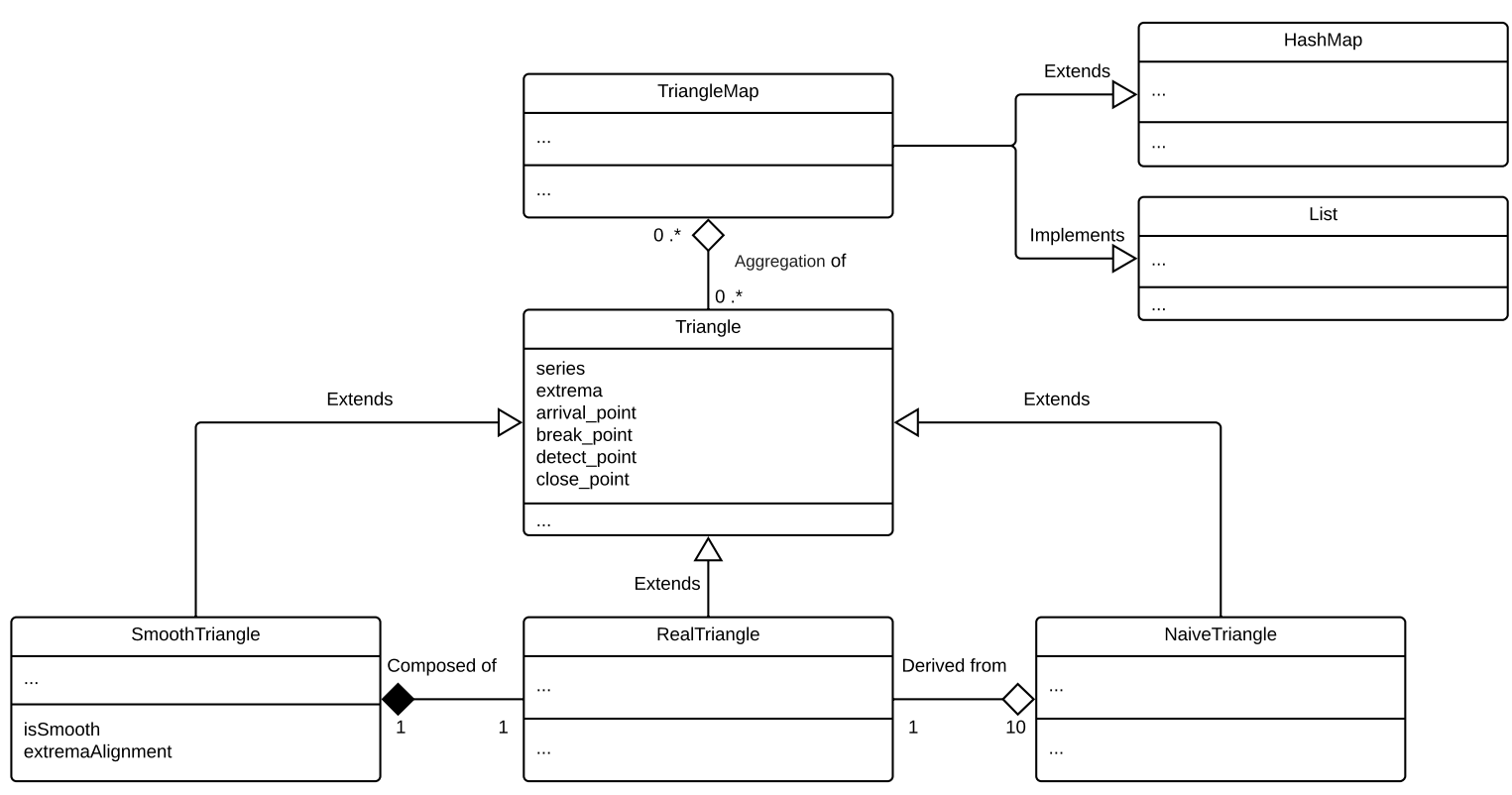

Figure 3.6: Triangle Class Diagram

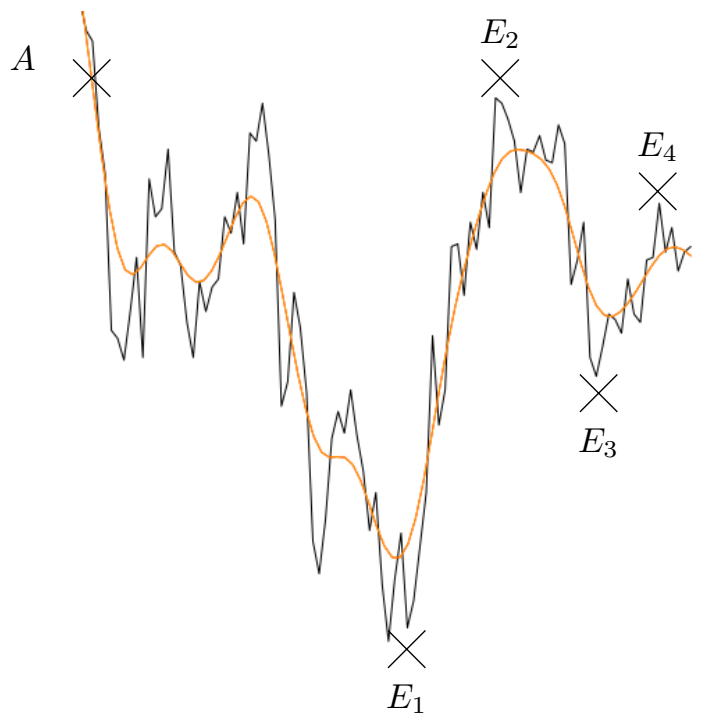

Figure 3.7: TBot Smoothness and Alignment Detection Example

esoteric software development terminology. Within this Section, detail is shown at a high level in English like pseudo code; the real code is considerably more complex, partly to increase computational speed, but it is not feasible to explain this in great detail within the thesis and most of this detail is less relevant to a conceptual understanding. This is a trade-off between high level conceptual understanding and precision, where the former takes precedence to communicate the ideas. The scanning algorithm is introduced in the following paragraphs and the relevant details are explained in pseudo code function based Algorithm listings 3.1 to 3.4. The core 


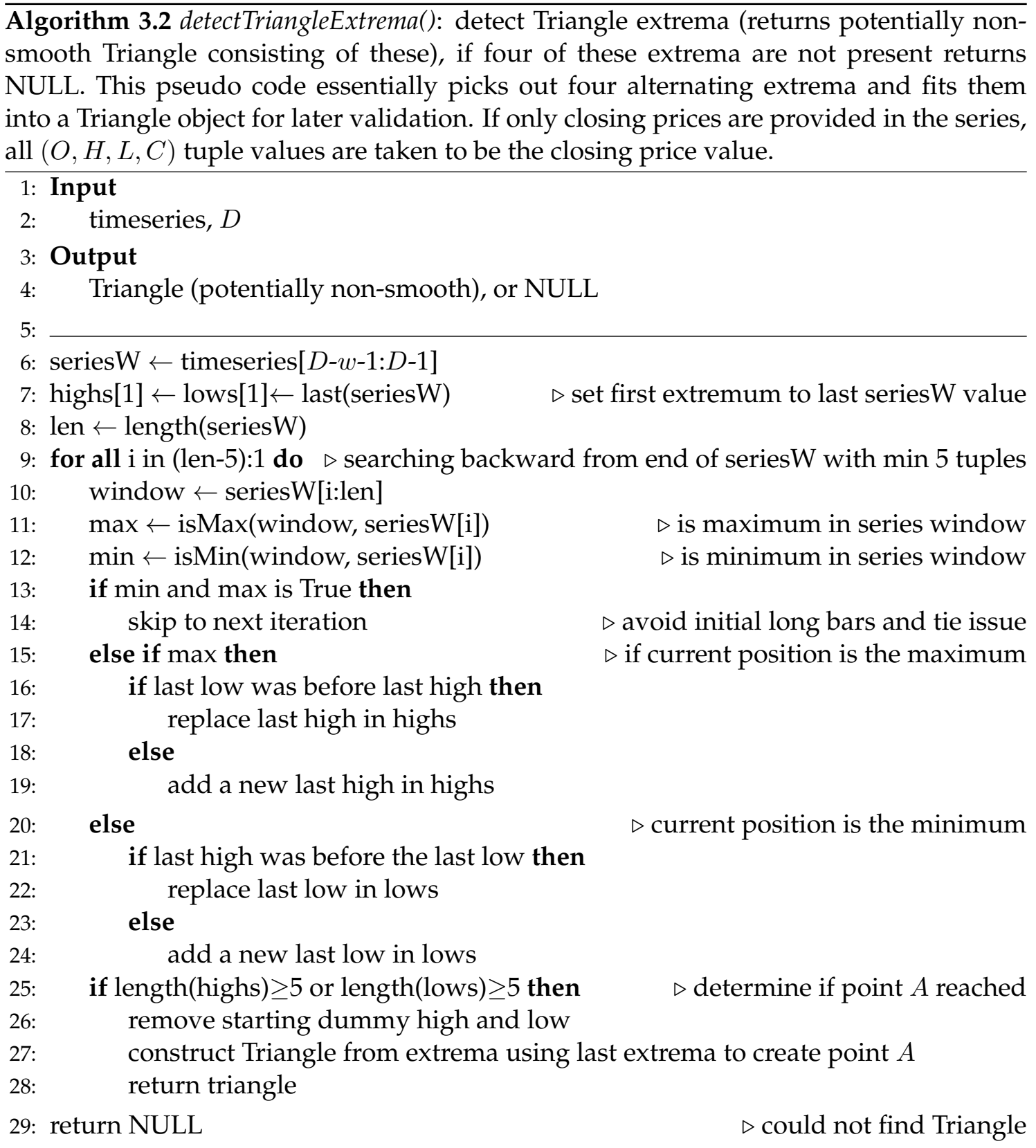

detection method starts from function call detectTriangle() in Algorithm 3.1 which calls the other functions to find a single Triangle pattern, if available, for each scan step. For the smaller functions used by this pseudo code, these are defined in English as:

- length(): determines the length of a time series, returned as an integer;

- isMax(): whether the value given is the maximum in a time series, returned as a boolean;

- isMin(): whether the value given is the minimum in a time series, returned as a boolean;

- last(): gets the last OHLC time series price, returned as a $(O, H, L, C)$ tuple;

- $\operatorname{diff():}$ vector absolute difference between integer values, returned as a vector of 


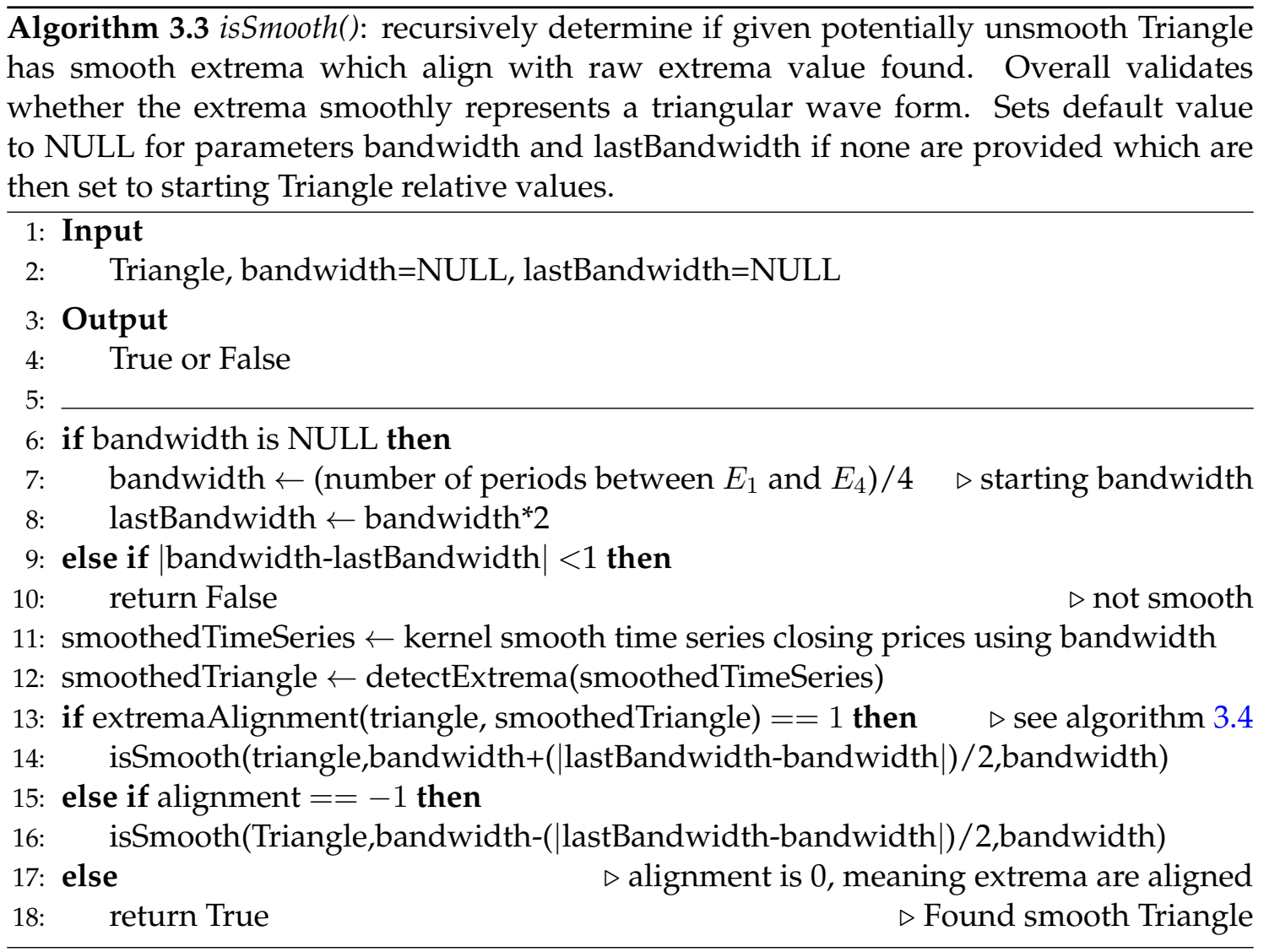

$\overline{\text { Algorithm 3.4 extremaAlignment(): determine whether a Triangle and SmoothedTriangle }}$ have sets of extrema which are aligned. This returns 0 if they are considered aligned, 1 if the smooth triangle extrema are aligned off to the right, -1 if the smooth triangle extrema are aligned off to the left. Currently this only uses $E_{1}$ to align which is rather rough but sufficient to overall help pick a kernel bandwidth.

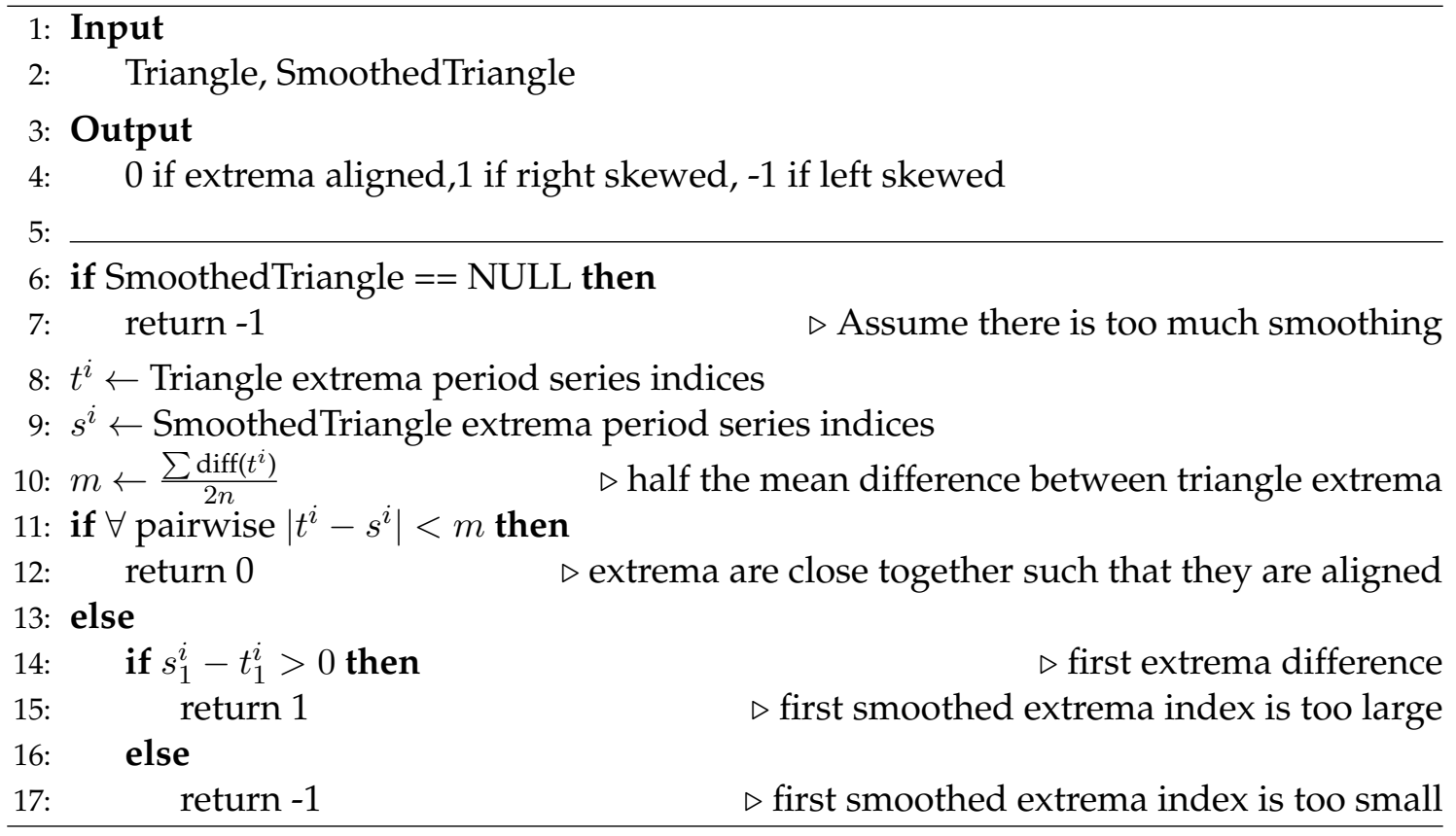


integers.

The Triangles found are stored in a TriangleMap object which is a hashmap hashed by tuple (type, $B, E_{1}, E_{2}, E_{3}, E_{4}$ ) to keep a unique store of Triangles. An interface of this class is also presented as a flat list which can be sliced by (type, $B$ ) tuple for results processing. This is used to manage the generation and processing of various results where views of the data can be accessed which can be processed using common $\mathrm{R}$ functions (several other more sophisticated data views and methods are used, but are not shown). Class diagram Figure 3.6 shows the structure of classes used to manipulate this data, showing only the core functionality relevant to the pseudo code. Triangle classes consist of three implemented types which extend the Abstract Triangle type and allow the use of various abstractions:

- RealTriangle which represents final captured Triangles used in data analysis, but may initially be Triangles which are not well formed due to not being smooth;

- SmoothTriangle used to determine spacing and extrema smoothness which contains the original RealTriangle and a smoothed time series (SmoothTriangle objects are discarded once RealTriangle objects are identified correctly);

- NaiveTriangle which represents randomly selected points in the original time series chosen to represent Triangles without a timing advantage; one RealTriangle generates 10 NaiveTriangles which refer back to the original RealTriangle from which they are derived.

The detection code scans a set time series window of size $w$ backwards from point $D$ searching for a single Triangle; this window scan is iteratively rolled forward one time series period at a time, repeating the process until the end of the series is reached to find all Triangles. A single detection step which scans one window is started by calling the detectTriangle() function on the given time series as described in algorithm 3.1. This function either finds a Triangle which is then stored in a TriangleMap or returns NULL to indicate that nothing has been found. The pseudo code explaining this process separates the SmoothedTriangle and RealTriangle for clarity, though in the real code there is a composite relationship as shown in Figure 3.6. In the pseudo code 'SmoothedTriangle' and 'RealTriangle', words starting with capitals, represent classes; whereas 'smoothedTriangle' and 'realTriangle' are camel case words representing class instances. Both types, capital case and camel case, are used to capture object based concepts in pseudo code.

The window scan algorithm contained within detectTriangle(), uses the function detectTriangleExtrema() in algorithm 3.2 to scan backward from point $D$ to determine alternating high and low points until four extrema are found to lock in point $A$. If an alternating set of extrema is found, a potentially unsmooth Triangle object is created from these, from which the algorithm determines if the extrema are smooth enough to be considered a tradable dampened wave type as illustrated in Figure1.1. If the Triangle 
is considered smooth, the algorithm then determines the break point $B$ (Up or Down type verbs) and subsequent trade close $C$. The smoothness evaluation can accommodate the Triangle size by determining the best kernel bandwidth, which it does by dynamically adapting the bandwidth size using a recursive binary search.

The binary search on each recursive search iteration smooths the window of time series closing prices from $A$ to $D$, then reuses the detectTriangleExtrema() function seen in 3.2 to determine whether a smooth Triangle can be found in the series window; each time the search is narrowed, trying to match the smooth and unsmoothed triangles until a match is found or the kernel bandwidth becomes too small.

The algorithm determines whether the extrema of the unsmooth realTriangle and smoothed smoothTriangle extrema are aligned such that they represent the same triangular shape as expressed in algorithm extremaAlignment 3.4 and as demonstrated pictorially in Figure 3.7. The alignment comparison is done by comparing the extrema of the RealTriangle and SmoothTriangles types, evaluating whether all of $E_{1}, E_{2}, E_{3}$ and $E_{4}$ extrema pairs are aligned between each type. The measure used for extrema alignment is whether the extrema of the realTriangle and smoothTriangle are within half the mean time period difference between realTriangle extrema. Overall this is done recursively by adjusting the kernel bandwidth until either a smooth Triangle is matched or it is determined that this cannot be done.

Again, overall this can be seen in Figure 3.7 where the orange line shows the smoothed closing price time series as compared with the black line of closing prices, showing that the last four extrema of the smoothed prices approximately match with the four extrema $E_{1}$ to $E_{4}$ (the extrema marked $E_{1}$ to $E_{4}$ on Figure 3.7 are not on the black closing price line, as these represent the highs or lows from OHLC prices). Overall this is not meant to be absolutely exact, but rather to roughly filter out various converging extrema, such that that only the reasonably smooth Triangles remain which would be considered tradable. Though this works well, the method could be vastly improved; but further improvement in identifying overlapping Triangles lies beyond the scope of this thesis. The smoothness determination can be seen in function isSmooth() as seen in algorithm 3.3.

\subsection{Naive Trades}

Having defined standardised Triangle returns in terms of $h$ and $\tau$ under $\mathcal{R}=1$, a comparative Naive model is useful from which to understand effect size differences. A short term trader is attempting to time trade position entry and exit while managing risk. The trader wishes to optimise these entry and exit times to maximise the use of his perceived Triangle advantage and minimise losses. A good comparative model then is one without the timing provided by Triangles, which forms the basis for the Naive model idea. The principle is to create a comparative model which uses the same 
information but does not provide timing information about where to enter and exit the security. This is initially conceptualised under the idea of AMH (2.1), where I consider that there are a variety of competing methodologies under which market participants trade; through aspects of natural selection certain strategies are able to dominate (though these may not necessarily provide superior returns, there being a range of factors determining strategy survival). Considering these ideas, I artificially create the Naive strategy to compete with the Triangle strategy to measure the competitive advantage. Assumptions are minimal, in the end not even requiring an assumption of any type of market efficiency (2.1).

As background, Lo et al. (2000) constructs what he calls an Unconditional model under which various pattern based trading methods are tested for information value, and the other related literature generally copies this concept closely. However this Unconditional model has an embedded assumption that once returns are normalised using drift and volatility all equities have the same daily return distribution. This assumes that returns can be normalised such that they are identically distributed, and this represents returns under the efficient markets hypothesis. This construction is then used to create various statistical tests. While this is a standard accepted approach, I do not believe stocks can be normalised to create a comparable distribution, and I further consider that a null hypothesis of EMH as a starting position is too strong. Also the normalisation process uses the entire time period of each stock to calculate drift and volatility to produce standardised returns for each stock, and this again is direct data snooping.

However the central idea of a distribution of normalised comparative returns is useful, and I attempt to improve on this idea by eliminating data snooping and removing assumptions where possible, though this is a difficult problem to solve well. Instead I create a Naive model using ideas of competitive advantage from AMH in which it is irrelevant whether the Naive model produces abnormal returns or not. I then drop AMH entirely, since I only need to compare a single competitive advantage, and a wider theory of market efficiency is no longer required.

To form the Naive model I assume individual stocks cannot be normalised universally and compared with an underlying knowledge of how their return is distributed. I assume such generated returns are not reliably comparable and so irrelevant (they are also not actual returns: see Table A.2). The objective is instead to measure the effect size difference that may exist due to trade timing by using a sampling method. However this is done differently for GBPUSD trial data and US equities. In general a comparison is made of Triangle $h$ values with the time taken for the same security to reach $\pm h$ for random entry points, for the trial data and equities.

Naive based procedure makes few assumptions about how the security price evolves, though to account for changing security structure across time the volatility for each 


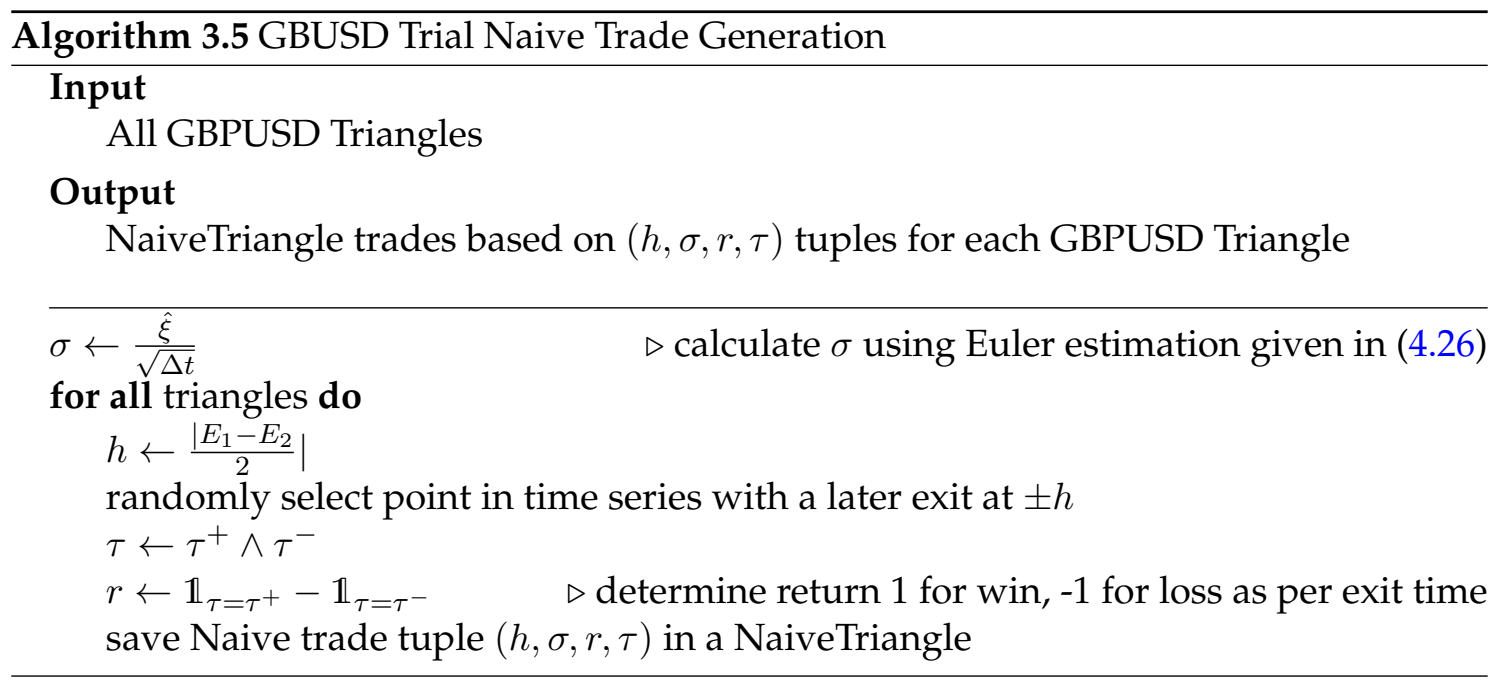

Triangle is used to normalise returns. For comparison I use the underlying null hypothesis that the Naive model first passage returns normalised by volatility are comparable to those for the Triangles for the same security. Overall I also assume that by combining the set of Naive returns with Triangle returns that their overall distributions are comparable. This is perhaps rather weak; but I argue it provides a stronger and more realistic alternative comparison to standard EMH return comparisons by being specific in using the same stock and the same standardisation. It does require trust in the abstraction of only looking for a comparative advantage without a strong overarching market theory. But the alternative of comparing with a logically unprovable theoretical model, though well researched and an interesting theory, is not a better solution. I make no assumptions as to whether Naive trades produce abnormal returns or not: the point is irrelevant, only the differences being important for this study.

For the trial using GBPUSD data, for each Triangle the following is done: a measurement of $h$ is taken from the Triangle and the direction of BUY or SELL is recorded. Then a random entry point is selected from the time series, at which point the same BUY or SELL is made with appropriate leverage such that $\mathcal{R}=1$ using $k=1$ for the Naive trade to standardise the return amount to \pm 1 . The time series is then walked forward from this point simulating a trade until either the $+h$ price level is hit for a Win or the $-h$ price level is hit for a Loss. The time taken $\tau$ for the first passage, $h$ and prior volatility at the entry point are recorded for the Naive trade similarly to Triangle trades. This is done 10 times for all Triangles, where each iteration is described by algorithm 3.5.

For US Equities the process is adjusted for multiple time series. A similar process is applied but within each stock time series individually. However with stocks the time series is often shorter, and where the $\pm h$ price level change is not met a fraction of $r$ is recorded. Also volatility is calculated as in (3.10) for the previous 100 days as for Triangles. This process is detailed in algorithm 3.6 which is done 10 times for each Triangle. 


$$
\begin{aligned}
\sigma & =\sqrt{\frac{1}{n-1} \sum_{i=1}^{n}\left(r_{i}-\bar{r}\right)} \text { where } r_{i}=\ln \left(\frac{P_{i}}{P_{i-1}}\right) \quad \text { and } \bar{r}=\frac{r_{1}+r_{2}+. .+r_{n}}{n} \\
n & =100 \text { day lookback period. }
\end{aligned}
$$

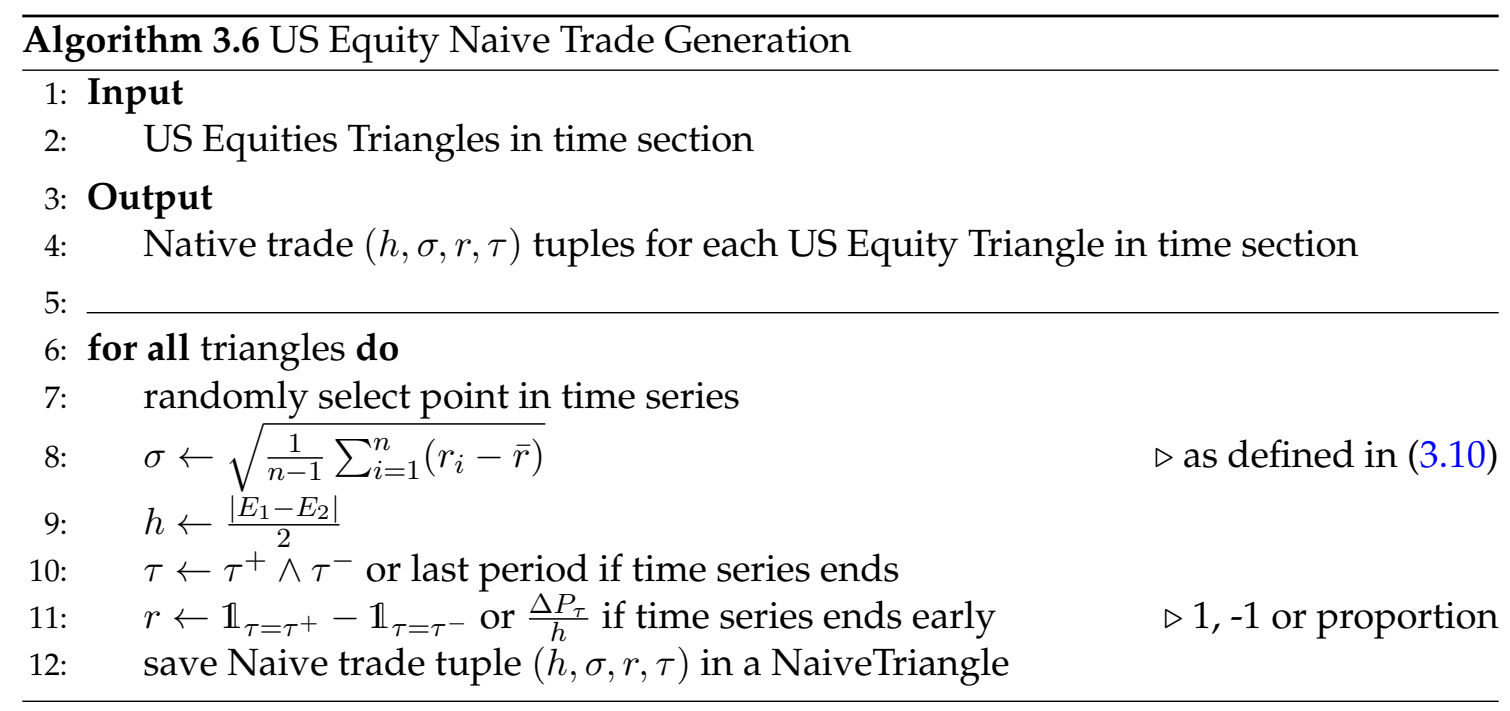

\subsection{Results Modelling}

Using these methods results are captured for ES (E-mini futures Appendix D), GBPUSD (spot currency Appendix C) and US equities (Appendix B). This generates Triangle and Naive results for each type of security which can then be analysed for differences to evaluate potential competitive timing advantages. Specially these include $(h, \sigma)$, considered determined prior to point $B$ before the trade starts; and $(\tau, r)$ which occur over segment $B C$ during the trade holding period. The aim is that this produces realistic results under which to evaluate trading. Given the evaluation as price level based trading, this has required development of modelling methods covered by the following chapters to explore the nature of the results.

Data snooping and various biases are rife in the trading literature and yet some realistic data is required for iterative development, which runs the risk of repeating such mistakes. Often the literature develops models from the data, then uses that same data to validate the model, which is clearly is not a sound research methodology. It seems more ethical to carefully think through how data is used and report this, so as to be clear that the selection of model has not involved data mining.

Carver (2015) discusses this and recommends the use of fake data, set up to contain the conditions required, then only test against real data when models are fully developed. To an extent, this avoids various data mining issues. Using this as a cue, I firstly use Brownian motion data to develop and test the algorithm, then use ES and GBPUSD trial 
time series data to develop realistic models to evaluate these returns. Real datasets are required to flush out realistic issues with models that may not be uncovered using fake data, particularly as the ideas are relatively new. The full models are then run on US Equities which maximises the avoidance of biases to obtain clean results. Additionally using single time series data for trials is significantly simpler to develop models against, rather than the thousands of time series that the US Equity data contains.

Using this trial approach, Structural and Intensity based methods are iteratively developed to evaluate the response of the conditional Triangle strategies compared to the Naive strategy. The objective of the modelling is to determine better ways to evaluate returns for this particular case. In the Structural and Intensity based chapters the trial data is referred to briefly, but a fuller description of trial development is given in Appendix C. This provides direct examples within the modelling chapters while avoiding saturation with excessive trial information.

The Structural method uses Brownian motion to estimate conditional changes in price structure; however, this was not pursued in depth due to difficulty in modelling short term price changes. The Intensity approach, as used with credit risk models, is a clear break with the finance literature tradition which uses predominantly continuous time stochastic processes.

For the Intensity approach, instead of trying to infer a structure of price changes when the trade breaks, the method interprets the results of changes as non-homogeneous marked Poisson process arrival times. However, though this modelling approach can be used to fit the results well, a difficulty is that interpreting results using this model is not clear, partly as the goodness of fit testing is somewhat unclear.

So a third approach was developed which uses bootstrapping to provide a 10 day constrained return based confidence interval. This method I call 'Effect Size Heuristics' which has the advantage of making virtually no modelling assumptions and providing a clear intuitive interpretation. No Trial data was used to develop this heuristic method due to its simplicity.

The following three chapters describe these modelling methods in detail, though only the Intensity and Effect Size Heuristic methods are applied to the US Equity data. Each method applies constraints and reasoning in order to measure the evidence for short term momentum, as opposed to longer term returns which are more exposed to wider systematic effects. 


\section{Chapter 4}

\section{Structural Model}

The aim of this chapter is to estimate structural parameters so as to consider the nature of prices after conditioning on Triangles where these are considered to behave as continuous time stochastic process. The intention is to estimate these parameters based on the development of price representing Triangle trading events over time series segments $B C$ as seen in Figure 4.1. This is in contrast to the usual use of such processes to model the entire time series. If a Triangle event has an effect on future price, then price conditional on these events will presumably show a different underlying structure to the Naive case. Ideally a stochastic process can be estimated from the distribution of first passage times which can be fitted and compared to facilitate this. Using the ideas of periodic returns extrapolated to continuous time motivates the use of Geometric Brownian Motion or Brownian Motion as suggested in Section 2.1.3.

To simplify the entry point to this analysis, rather than using the combined first passage above and below, I start by exploring only the first passage above using Brownian motion. Towards this, I derive the first passage above stopping time distribution, and apply this to short term results using maximum likelihood to estimate scale and drift parameters. The attraction of a structural approach is that it comes with a plethora of well understood financial methods and established mathematical frameworks to further understand results. See appendix $C$ for more detailed information on the GBPUSD data and trial of these methods.

Ideally the Structural approach would have gravitated towards estimating parameters using first passage distribution times above and below with Geometric Brownian motion or other more sophisticated models. The general estimation method was however not particularly successful; within this failure, some exploratory work shows features of the underlying data, which motivates the use of alternative comparative models.

Consider then price to evolve as an Itô process as given by the stochastic differential equation 


$$
d X_{t}=\mu\left(X_{t}, t\right) d t+\sigma\left(X_{t}, t\right) d B_{t}
$$

where $d B_{t} \sim \mathcal{N}(0, d t), B_{t}$ being a standard Wiener process. In relation to securities, $\mu$ and $\sigma$ represent drift and volatility respectively. See Kloeden and Platen (1992) for further detail of numerical approaches to stochastic differential equations.

With GBPUSD, for a simple starting model we assume over the short term trade holding period that price structure does not vary with time; and so we assume constant $\mu$ and $\sigma$, such that prices evolve according to scaled Brownian Motion with drift as seen in (4.2). We can then estimate the parameters of drift given by $\mu$ and volatility given by $\sigma$ to define our process from the trade break $B$ to close $C$.

$$
d X_{t}=\mu d t+\sigma d B_{t}
$$

However security prices are more commonly modelled as Geometric Brownian Motion with drift and scale as seen in (2.3), typically expressed as

$$
d S_{t}=\mu S_{t} d t+\sigma S_{t} d B_{t}
$$

Geometric Brownian Motion is generally a more palatable model for securities because prices are positive. However use of Brownian Motion over a very short term period is appropriate since over this period (in the GBPUSD trial 60 minutes) the modelled Brownian Motion is highly unlikely to go below zero, and so negative prices are not an issue. Additionally, in the trial for GBPUSD partly due to efficient markets and for comparison/testing purposes I assume that drift is zero. Bearing in mind this is an initial exploration of the structural concept, these simplifications are acceptable as a starting point which allow exploration of the ideas with minimal complexity.

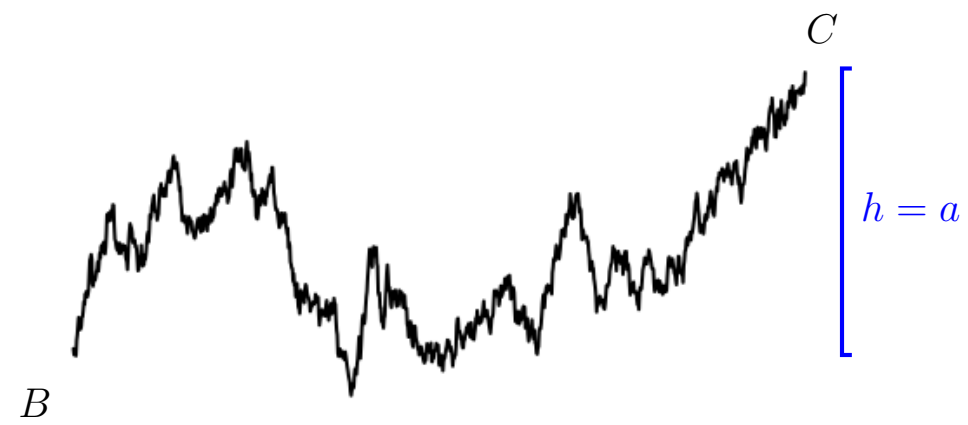

Figure 4.1: Brownian Motion First Passage Example

Relating this theory back to Triangles, Brownian Motion can be used to approximate the security price path over segments of $B C$, for example the sample path for the security 
may display the price plotted against time as seen in Figure 4.1. It is then possible that this can be represented as a continuous time random process and parameters can be estimated to simulate a sample path with similar characteristics that represents the effects of price conditional on Triangles.

Hence, once the structural model is chosen, in this case Brownian Motion, a model for the first passage distribution can be derived from the sample of $B C$ segments via Maximum likelihood to estimate the drift and scale parameters using $(r, h, \tau)$ sample values. To empirically compare results, I employ both a constrained Naive case and a Brownian Motion Euler based estimation of the full time series.

In the following sections I derive the Brownian Motion first passage above distribution, estimate the parameters and devise a method to explore the fit visually over a constrained surface. I then evaluate these results toward the development of alternative methods.

\subsection{Brownian Motion First Passage Derivation}

Consider the equations derived from a standard normal distribution as

$$
Y=\mu+\sigma Z, \quad Z \sim \mathcal{N}(0,1), \quad \Longrightarrow Y \sim \mathcal{N}\left(\mu, \sigma^{2}\right)
$$

for which the Moment Generating Function is $M_{Y}(\theta)=E\left(e^{\theta Y}\right)=e^{\mu \theta+\frac{1}{2} \sigma^{2} \theta^{2}}$.

Similarly for Brownian Motion with drift and scale

$$
\begin{aligned}
& X_{t}=\mu t+\sigma B_{t} \quad X_{t} \sim \mathcal{N}\left(\mu t, \sigma^{2} t\right) \\
& M_{X}(\theta)=E\left(e^{\theta X_{t}}\right)=e^{t\left(\mu \theta+\frac{1}{2} \sigma^{2} \theta^{2}\right)} .
\end{aligned}
$$

Following this, consider the Laplace exponent of Brownian Motion with drift $\mu$ and scaling factor $\sigma$ as $\psi(\theta)=\mu \theta+\frac{\sigma^{2}}{2} \theta^{2}$ to form expected value

$$
\begin{aligned}
E_{x}\left(e^{\theta\left(X_{t}-x\right)}\right) & =e^{t \psi(\theta)} \quad \text { where } x \text { is the starting point of } X_{t} \\
e^{-t \psi(\theta)} E_{x}\left(e^{\theta\left(X_{t}-x\right)}\right) & =1 \\
E_{x}\left(e^{-t \psi(\theta)} e^{\theta\left(X_{t}-x\right)}\right) & =1 .
\end{aligned}
$$

Since (4.6) holds for all $t \geq 0$, then by Doob's optional stopping theorem the following holds where $X_{t}$ hits at stopping level above $a$; such that we can replace $X_{t}$ by $X_{\tau_{a}}$ and time $t$ with $\tau_{a}$ as 


$$
\begin{aligned}
E_{x}\left(e^{-\psi(\theta) \tau_{a}} e^{\theta\left(X_{\tau_{a}}-x\right)}\right) & =1 & & \text { where } \tau_{a}=\inf \left\{t>0: X_{t} \geq a\right\} \\
E_{x}\left(e^{-\psi(\theta) \tau_{a}} e^{\theta(a-x)}\right) & =1 & & \text { replace } X_{\tau_{a}} \text { with non-random stopping level } a \\
e^{\theta(a-x)} E_{x}\left(e^{-\psi(\theta) \tau_{a}}\right) & =1 & & \\
E_{x}\left(e^{-\psi(\theta) \tau_{a}}\right) & =e^{-\theta(a-x)} & &
\end{aligned}
$$

noting that $a>x$ since $a$ is the stopping level above.

In order to solve this, define

$$
\begin{aligned}
\Phi(\lambda) & =\sup (\theta: \psi(\theta)=\lambda) \quad \text { such that } \theta \text { is the largest root } \\
\Rightarrow \psi(\Phi(\lambda)) & =\lambda .
\end{aligned}
$$

Replace $\theta$ with $\Phi(\lambda)$

$$
\begin{aligned}
E_{x}\left(e^{-\psi(\theta)) \tau_{a}}\right) & =e^{-\theta(a-x)} \\
E_{x}\left(e^{-\psi(\Phi(\lambda)) \tau_{a}}\right) & =e^{-\Phi(\lambda)(a-x)} \\
E_{x}\left(e^{-\lambda \tau_{a}}\right) & =e^{-\Phi(\lambda)(a-x)} \\
\int_{0}^{\infty} e^{-\lambda t} \mathbb{P}\left(\tau_{a} \in d t\right) & =e^{-\Phi(\lambda)(a-x)},
\end{aligned}
$$

where $\mathbb{P}$ is the probability of crossing at $\tau_{a}$ within $d t$.

Using the Laplace exponent find the roots in order to find $\Phi(\lambda)$

$$
\begin{aligned}
\psi(\theta) & =\mu \theta+\frac{\sigma^{2}}{2} \theta^{2}=\lambda \\
0 & =\mu \theta+\frac{\sigma^{2}}{2} \theta^{2}-\lambda \\
\Longrightarrow \Phi(\lambda) & =\frac{-\mu+\sqrt{\mu^{2}+2 \lambda \sigma^{2}}}{\sigma^{2}} .
\end{aligned}
$$

Then substitute $\Phi(\lambda)$ into (4.9) to form

$$
\int_{0}^{\infty} e^{-\lambda t} \mathbb{P}\left(\tau_{a} \in d t\right)=e^{-(a-x)\left(\frac{-\mu+\sqrt{\mu^{2}+2 \lambda \sigma^{2}}}{\sigma^{2}}\right)} .
$$

So that for standard Brownian Motion where $\mu=0$ and $\sigma=1$ starting at $x=0$ we have by stationarity of sample paths 


$$
\begin{aligned}
\int_{0}^{\infty} e^{-\lambda t} \mathbb{P}\left(\tau_{a} \in d t\right) & =e^{-a \sqrt{2 \lambda}} \\
p(t) & =\mathcal{L}^{-1}\left(e^{-a \sqrt{2 \lambda}}\right) \quad \text { where } \mathcal{L}^{-1} \text { is the inverse laplace transform } \\
& =\frac{1}{\sqrt{2 \pi t^{3}}} e^{\frac{-a^{2}}{2 t}}
\end{aligned}
$$

This gives the first passage distribution for standard Brownian Motion as seen in Karatzas and Shreve (1988).

However the distribution given in (4.12) is for standard Brownian Motion, lacking drift and scale. I now add drift term $\mu$ where $X_{t}=\mu t+B_{t}$ and apply a change of measure. Using the ratio

$$
E_{\mathbb{P}}\left(\frac{d \mathbb{P}^{v}}{d \mathbb{P}^{p}} \mid \mathcal{F}_{t}\right)=1
$$

where $\mathcal{F}_{t}$ is the filtration at time $t$, it is clear that the following satisfies (4.13)

$$
\begin{aligned}
\frac{d \mathbb{P}_{v}}{d \mathbb{P}} \mid \mathcal{F}_{t} & =e^{v X_{t}-\psi(v) t} \\
E_{\mathbb{P}}\left(\frac{d \mathbb{P}_{v}}{d \mathbb{P}} \mid \mathcal{F}_{t}\right) & =E_{\mathbb{P}}\left(e^{v X_{t}-\psi(v) t}\right) \\
& =e^{-\psi(v) t} E_{\mathbb{P}}\left(e^{v X_{t}}\right) \\
& =e^{-\psi(v) t} e^{\psi(v) t}=1,
\end{aligned}
$$

$\therefore \frac{d \mathbb{P}_{v}}{d \mathbb{P}} \mid \mathcal{F}_{t}=e^{v X_{t}-\psi(v) t}$ is a valid change of measure. Hence under the new measure $E_{\mathbb{P}_{v}}\left(e^{\theta X_{t}}\right)=e^{\psi_{v}(\theta) t}$ where $\psi_{v}(\theta)=\frac{1}{2} \theta^{2}$ is the Laplace exponent of standard Brownian Motion under the new measure $\mathbb{P}_{v}$. Indeed under $\mathbb{P}_{v}$

$$
\begin{aligned}
E_{\mathbb{P}_{v}}\left(e^{\theta X_{t}}\right) & =E_{\mathbb{P}}\left(e^{\theta X_{t}} \frac{d \mathbb{P}_{v}}{d \mathbb{P}} \mid F_{t}\right) \\
& =E_{\mathbb{P}}\left(e^{\theta X_{t}} e^{v X_{t}-\psi(v) t}\right) \\
& =e^{-\psi(v) t} E_{\mathbb{P}}\left(e^{X_{t}(\theta+v)}\right) \\
& =e^{-\psi(v) t} e^{\psi(\theta+v) t} \\
& =e^{[\psi(\theta+v)-\psi(v)] t}
\end{aligned}
$$




$$
\begin{aligned}
\text { Since } E_{\mathbb{P}_{v}}\left(e^{\theta X_{t}}\right) & =e^{\psi_{v}(\theta) t}=e^{[\psi(\theta+v)-\psi(v)] t}=e^{\frac{1}{2} \theta^{2}} \\
\Longrightarrow \psi(\theta+v)-\psi(v) & =\frac{1}{2} \theta^{2} \\
\mu(\theta+v)+\frac{1}{2}(\theta+v)^{2}-\left(\mu v+\frac{1}{2} v^{2}\right) & =\frac{1}{2} \theta^{2} \\
\mu \theta+\theta v & =0 \\
-\mu & =v .
\end{aligned}
$$

Hence the drift component has been accounted for under the new measure where

$$
\begin{array}{ll}
X_{t}=\mu t+B_{t} & \text { under } \mathbb{P} \\
X_{t}=B_{t} & \text { under } \mathbb{P}^{v} .
\end{array}
$$

Noting the following

$$
\frac{d \mathbb{P}_{v}}{d \mathbb{P}}\left|\mathcal{F}_{t}=e^{v X_{t}-\psi(v) t}, \quad \frac{d \mathbb{P}}{d \mathbb{P}_{v}}\right| \mathcal{F}_{t}=e^{-v X_{t}+\psi(v) t}
$$

$$
\begin{array}{rlr}
E_{\mathbb{P}_{v}}\left(e^{-\lambda \tau_{a}}\right) & =E_{\mathbb{P}}\left(e^{-\lambda \tau_{a}} \frac{d \mathbb{P}_{v}}{d \mathbb{P}} \mid \mathcal{F}_{t}\right) \\
E_{\mathbb{P}}\left(e^{-\lambda \tau_{a}}\right) & =E_{\mathbb{P}_{v}}\left(e^{-\lambda \tau_{a}} \frac{d \mathbb{P}}{d \mathbb{P}_{v}} \mid \mathcal{F}_{t}\right) \\
& =E_{\mathbb{P}_{v}}\left(E_{\mathbb{P}_{v}}\left(e^{-\lambda \tau_{a}} \frac{d \mathbb{P}}{d \mathbb{P}_{v}} \mid \mathcal{F}_{\tau_{a}}\right)\right) \quad \text { Law of Iterated Expectation } \\
& =E_{\mathbb{P}_{v}}\left(e^{-\lambda \tau_{a}} e^{-v X_{\tau_{a}}+\psi(v) \tau_{a}}\right) & \\
& =e^{-v a} E_{\mathbb{P}_{v}}\left(e^{-\lambda \tau_{a}+\psi(v) \tau_{a}}\right) & \\
& =e^{-v a} E_{\mathbb{P}_{v}}\left(e^{-p \tau_{a}}\right) & \text { where } p=\lambda-\psi(v) .
\end{array}
$$

Following from 4.9 we have

$$
\begin{aligned}
E_{\mathbb{P}_{v}}\left(e^{-p \tau_{a}}\right) & =e^{-\Phi_{v}(p) a} \\
\Phi_{v}(\lambda)=\sup \left(p: \psi_{v}(p)\right. & =\lambda) \Longrightarrow \psi_{v}\left(\Phi_{v}(\lambda)\right)=\lambda \\
\lambda=\psi_{v}^{-1}(p) & =\Phi_{v}(p) .
\end{aligned}
$$


Find the solution under the new measure as

$$
\begin{aligned}
p & =\psi_{v}(\lambda)=\psi(\lambda+v)-\psi(v) \\
p & =\mu(\lambda+v)+\frac{1}{2}(\lambda+v)^{2}-\mu v-\frac{1}{2} v^{2} \\
p & =\mu \lambda+\mu v+\frac{1}{2} \lambda^{2}+\frac{1}{2} v^{2}+\lambda v-\mu v-\frac{1}{2} v^{2} \\
p & =\frac{1}{2} \lambda^{2} \\
\Longrightarrow \lambda & =\sqrt{2 p}=\sqrt{2(\lambda-\psi(v))} \\
& =\sqrt{2\left(\lambda+\frac{1}{2} \mu^{2}\right)}=\Phi_{v}(p) \quad \text { as seen in (4.20). }
\end{aligned}
$$

This result can be used to solve the expectation under $\mathbb{P}$ seen in (4.19) using the inverse Laplace transform to extract the first passage time distribution as

$$
\begin{aligned}
E_{\mathbb{P}}\left(e^{-\lambda \tau_{a}}\right) & =e^{-v a} E_{\mathbb{P}_{v}}\left(e^{-p T_{a}}\right) \\
& =e^{-v a} e^{-\Phi_{v}(p) a} \\
p(t) & =\mathcal{L}_{\lambda_{v}}^{-1}\left(e^{-v a} e^{-a \sqrt{2\left(\lambda+\frac{1}{2} \mu^{2}\right)}}\right) \\
& =e^{\mu a} \mathcal{L}_{\lambda_{v}}^{-1}\left(e^{-a \sqrt{2\left(\lambda+\frac{1}{2} \mu^{2}\right)}}\right) \\
& =e^{\mu a}\left(\frac{a e^{\frac{-a^{2}}{2 t}-\frac{1}{2} \mu^{2}}}{\sqrt{2 \pi t^{3}}}\right) \\
& =\frac{a e^{\frac{-(a-\mu t)^{2}}{2 t}}}{\sqrt{2 \pi t^{3}}} .
\end{aligned}
$$

Which is the solution for the Brownian Motion first passage density above with scale $\mu$ and $\sigma=1$ as seen in Borodin and Salminen (2002).

Consider then the first passage time for Brownian motion with scale $\sigma \in \mathcal{R}_{>0}$ and drift $\mu$ where we effectively divide by $\sigma$ to normalise then solve for $X_{t \sigma}$,

$$
\begin{aligned}
\text { as previously } & \tau_{a}=\inf \left\{t>0: X_{t}=\mu t+B_{t} \geq a\right\} \quad \text { where } \sigma=1 \\
\text { so } \quad & \hat{\tau}_{a}=\inf \left\{t>0: X_{t}=\mu t+\sigma B_{t} \geq a\right\} \quad \text { where } \sigma \in \mathcal{R}_{>0} \\
\Longrightarrow \quad \hat{\tau}_{a}=\inf \left\{t>0: \frac{X_{t}}{\sigma}=\frac{\mu t}{\sigma}+B_{t} \geq \frac{a}{\sigma}\right\} . &
\end{aligned}
$$

Hence substitute drift $\frac{\mu}{\sigma}$ and first passage level $\frac{a}{\sigma}$ into the first passage distribution given by (4.22) where $\hat{\tau_{a}} \stackrel{d}{=} \tau$, resulting in

$$
p(t)=\frac{\frac{a}{\sigma} \exp \left(\frac{-\left(\frac{a}{\sigma}-\frac{\mu t}{\sigma} t\right)^{2}}{2 t}\right)}{\sqrt{2 \pi t^{3}}}=\frac{a \exp \left(-\frac{(a-\mu t)^{2}}{2 \sigma^{2} t}\right)}{\sigma \sqrt{2 \pi t^{3}}} .
$$




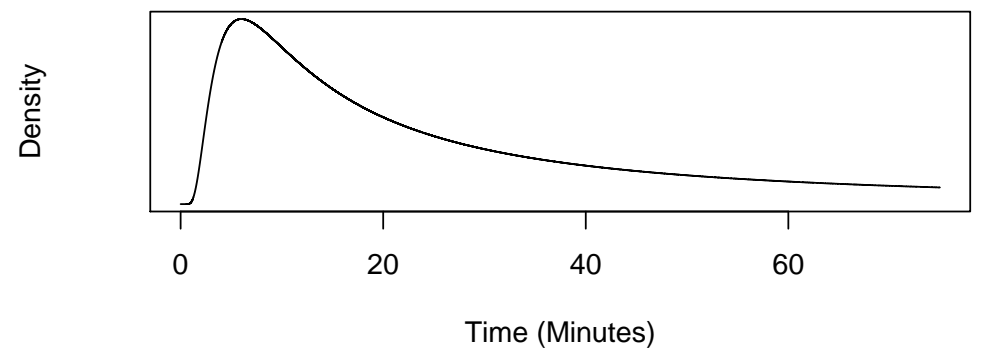

Figure 4.2: Typical GBPUSD Brownian Motion First Passage Time $h=12$ (pips)

Visually this produces a distribution of first passage times for segment $B C$ as seen in Figure 4.1, where it can be seen that this is highly skewed to the right. This provides two potential uses: an intuitive understanding of the distribution of how long trades take to reach price level $h$ over segment $B C$, and a way to estimate distribution parameters using the product of likelihoods.

The derivation of the first passage below is analogous to the first passage above, but is not shown for brevity. The combined single sided first passage distribution above or below from point $B$ to $C$, to change in price $a$, is then

$$
p(t)=\frac{|a| \exp \left(-\frac{(a-\mu t)^{2}}{2 \sigma^{2} t}\right)}{\sigma \sqrt{2 \pi t^{3}}} .
$$

\subsection{Brownian Motion First Passage Estimation}

The derivation of the probability density given in equation (4.24) does so for infinite time. This is not practical as we only want first passage times for a constrained interval of time after which it is likely that the Triangle no longer has an effect. There are also practical computational limits of searching very large time series for tens of thousands of crossing times, as this turns out to be extremely time consuming. Also under this trial some Triangles never reach the first passage time so some constraint is forced on the estimation. Instead I constrain the time interval using conditional probability to obtain events which occur conditionally within time $t_{c}$. This is clearly not ideal but it is a starting practical estimation method for initial analysis. This results in a time constrained truncated distribution with only events where $t<t_{c}$ of 


$$
\begin{gathered}
f_{c}(t)=\frac{\frac{|a| \exp \left(-\frac{(a-\mu t)^{2}}{2 \sigma^{2}}\right)}{\sigma \sqrt{2 \pi t^{3}}}}{\int_{0}^{t_{c}} \frac{|a| \exp \left(-\frac{(a-\mu t)^{2}}{2 \sigma^{2} t}\right)}{\sigma \sqrt{2 \pi t^{3}}} d t}=\frac{f(t)}{F\left(t_{c}\right)} \\
\text { where } \quad \int_{0}^{t_{c}} f_{c}(t) d t=\frac{\int_{0}^{t_{c}} f(t) d t}{F\left(t_{c}\right)}=\frac{F\left(t_{c}\right)}{F\left(t_{c}\right)}=1 .
\end{gathered}
$$

Assuming independence, the same underlying structural model, and denoting the likelihood by $\mathcal{L}$ and its logarithm by $\ell$, the maximum likelihood can be used to estimate $\mu$ and $\sigma$ by

$$
\begin{aligned}
\mathcal{L}(\mu, \sigma \mid t, h) & =\prod_{i=1}^{n} f_{c}\left(t_{i}, h_{i} \mid \mu, \sigma\right) \\
& =\prod_{i=1}^{n} f_{c}\left(t_{i} \mid h_{i}, \mu, \sigma\right) f_{c}\left(h_{i} \mid \mu, \sigma\right) \\
\ell(\mu, \sigma \mid t, h) & =\sum_{i=1}^{n} \ln \left[f_{c}\left(t_{i} \mid h_{i}, \mu, \sigma\right) f_{c}\left(h_{i} \mid \mu, \sigma\right)\right] .
\end{aligned}
$$

In the expression $f_{c}\left(t_{i} \mid h_{i}, \mu, \sigma\right)$ is the truncated stopping time density for one fixed height given by (4.26) and $f_{c}\left(h_{i} \mid \mu, \sigma\right)$ is the marginal empirical density of heights where $t \leq t_{c}$. Density $f\left(h_{i} \mid \mu, \sigma\right)$ does not have an exact solution available but can be estimated using a kernel density over the observed empirical values, where true parameter values of $\mu$ and $\sigma$ are considered embedded within the marginal distribution given by the kernel.

The likelihood given by (4.27) must then be solved numerically, as opposed to using a differential extremum approach, by using optimisation to search the space of all possible values of $\mu$ and $\sigma$ for the value that maximises the log likelihood using the kernel estimate of $f\left(h_{i} \mid \mu, \sigma\right)$. Standard $\mathrm{R}$ code is used to generate this kernel to produce a smooth density with only one maximum, to prevent optimisation finding a local maximum point. This is later checked by plotting the manifold and making sure the optimisation of $\mu$ by $\sigma$ converges the correct maximum.

Table 4.1: GBPUSD Triangle Brownian Motion First Passage Estimates

\begin{tabular}{rrrrr}
\hline & TTop Up & TTop Down & TBot Down & TBot Up \\
\hline$\sigma$ & 0.206 & 0.327 & 0.211 & 0.261 \\
$\mu$ & 0.000 & 0.000 & 0.000 & 0.000 \\
\hline
\end{tabular}

The results of estimation for Triangles and Naive trades are shown in tables 4.1 and 4.2 
Table 4.2: GBPUSD Naive Brownian Motion First Passage Estimates

\begin{tabular}{rrrrr}
\hline & TTop Up & TTop Down & TBot Down & TBot Up \\
\hline$\sigma$ & 0.167 & 0.158 & 0.178 & 0.167 \\
$\mu$ & 0.000 & 0.000 & 0.000 & 0.000 \\
\hline
\end{tabular}

respectively. The estimates for $\mu$ are close to zero, which does not appear realistic. This seems likely due to the nature of equation (4.24), as the negative exponential term may bias the estimation due to a large number of results occurring with $t \approx 0$ such that the estimation is unrealistically dominated by these values (potential wrong model problem). Curiously the estimates for $\sigma$ seem to be reflective of the magnitude of empirical returns as seen in Appendix C.5. This is intuitively unclear and so it was decided to investigate the data itself to understand the nature of the empirical results, which is explained below.

\subsection{Surface Exploration}

To explore the nature of my results, a surface is plotted of various empirical and theoretical representations of Triangle and Naive results along with a surface of a Brownian Motion estimation for the entire GBPUSD time series. This helps clarify the nature of $(r, \tau)$ results, but requires some manipulation to create comparative density magnitudes. Results are shown for TTop Up, and further detail is shown in Appendix C.

\subsection{Euler Estimation Baseline}

It is useful to establish a baseline of comparison over the entire time series to approximately check the estimation and to more intuitively understand how closely the structure assumed over the trading period holds. The estimation uses the entire time series and it is not realistic that this model would hold over this period; rather this is used to comparatively gauge how reasonable estimations are from the first passage based estimation of trading events. This is done to compare the Brownian Motion estimate over the whole period with the maximum likelihood piece-wise estimate over trade holding periods. The estimation process can be seen as

Stochastic Equation $\rightarrow$ Estimate Regression Coefficients $\rightarrow$ Derive Equation Parameters

For Brownian Motion as seen in 4.2 this results in estimation of parameters as 


$$
\begin{aligned}
X_{t+s}-X_{t} & \sim \mathcal{N}\left(\mu s, \sigma^{2} s\right), \quad \forall s>0 \\
\text { where } \quad s & =\Delta t, \quad t=t_{n} \quad \text { for } \quad t=\{0,1,2 \ldots\} \\
X_{t+s}-X_{t} & =d+v, \quad v \sim \mathcal{N}\left(0, \sigma^{2} \Delta t\right) \\
d & =\mu \Delta t, \quad \xi^{2}=\sigma^{2} \Delta t \\
\hat{\mu} & =\frac{\hat{d}}{\Delta t}, \quad \hat{\sigma}=\frac{\hat{\xi}}{\sqrt{\Delta t}} .
\end{aligned}
$$

This results in an estimation of $\hat{\mu}=0.000$ and $\sigma=0.173, \mu$ being extremely small and so virtually zero; $\mu \approx 0$ corroborates with the trial results seen in Tables 4.1 and 4.2.

In trialling these ideas it became apparent that it was not clear from estimation how close the assumed structure was to the underlying data distribution. Additionally the particularly long tails in both $h$ and stopping time $t$ make this hard to conceptualise. Since short term momentum is of principal interest, and the sample size of sections with large $h$ is too low to model well. Hence I plot a constrained surface to each view of the stopping time distribution for each of Brownian Motions, Triangles and Naive.

Consider for a single fixed $h$ the stopping time density of $\tau$ under time constraint $t_{c}$ where all $t \leq t_{c}$ as

$$
\begin{array}{rlrl}
\mathbb{P}\left(\tau \in d t \mid \tau \leq t_{c}, h\right) & =\frac{\mathbb{P}\left(\tau \in d t, \tau \leq t_{c} \mid h\right)}{\mathbb{P}\left(\tau \leq t_{c} \mid h\right)} & \\
& =\frac{\mathbb{P}(\tau \in d t \mid h)}{\mathbb{P}\left(\tau \leq t_{c} \mid h\right)} \quad \text { since all } t \leq t_{c} \\
& =\frac{f(t \mid h)}{\int_{0}^{t_{c}} f(x \mid h) d x} \quad \text { where } f \text { is the density derived in } 4.24 \\
\mathbb{P}\left(\tau \leq t_{c} \mid \tau \leq t_{c}, h\right) & =\frac{\int_{0}^{t_{c}} f(x \mid h) d x}{\int_{0}^{t_{c}} f(x \mid h) d x}=1 \quad \text { so forms constrained PDF within } t_{c} \text { conditional on } \mathrm{h} .
\end{array}
$$

Then divide constrained PDF into sections and calculate probability within each section, where $l$ and $u$ represent lower and upper bounds respectively

$$
\begin{aligned}
\mathbb{P}\left(t_{l}<\tau \leq t_{u} \mid \tau \leq t_{c}, h\right) & =\frac{\mathbb{P}\left(t_{l}<\tau \leq t_{u}, \tau \leq t_{c} \mid h\right)}{\mathbb{P}\left(\tau \leq t_{c} \mid h\right)} \\
& =\frac{\mathbb{P}\left(t_{l}<\tau \leq t_{u} \mid h\right)}{\mathbb{P}\left(\tau \leq t_{c} \mid h\right)} \quad \text { since all } t_{u} \leq t_{c} \\
\mathbb{P}\left(\tau \leq t_{c} \mid \tau \leq t_{c}, h\right) & =\frac{\int_{t_{l}}^{t_{u}} f(x \mid h) d x}{\int_{0}^{t_{c}} f(x \mid h) d x}=1 \quad \text { forming constrained probability section. }
\end{aligned}
$$

Similarly for all $h$ values in grid squares $h \in(a, b]$ and $\tau \in(l, u]$ find the mean probability in that grid square as 


$$
\frac{\sum_{i=1}^{n} \mathbb{P}\left(t_{l}<\tau \leq t_{u} \mid \tau \leq t_{c}, h_{i}\right)}{\operatorname{count}(h \in(a, b]))}, \quad \forall h_{i} \in(a, b]
$$

I do this iteratively for all $a, b$ and $l, u$ grid sections to form a grid of probability that sum to 1 . This creates an approximate joint probability mass function over these sections for comparative purposes.

For Triangle and Naive cases we can find constrained sums for each of the grid squares and divide by the total count to convert into an approximate probability mass function. In the Triangle case we select all Triangles that occur within upper bounds $t_{c}$ and $h_{c}$. In the Naive case we take each event from the bounded Triangles and randomly forward select from within the time series events which occur within $\left(0, t_{c}\right]$, this being all Naive events crossing above or below during the time interval $\left(0, t_{c}\right]$.

This creates a grid of comparable probability mass values which approximate a constrained joint probability grid square by grid square, which can be plotted against one another. This is approximate as it assumes an equal mass within each constrained $h t$ section which is a poor assumption and will be more inaccurate the smaller the time constraint. Hence the time constraint needs to be large enough to capture the majority of the probability mass for the distribution grids to be comparable. This is sufficient for an intuitive comparison to check for structural fit visually, as seen more fully in the GBPUSD trial discussed in Appendix C.

As a demonstration results of Brownian Motion can be seen in Figure 4.3 which shows the contained distribution of stopping times for TTop Up $h$ values if the distribution of first passage times was from Brownian Motion with $\mu=0$ and $\sigma=0.173$. The idea is to gauge how relevant the estimation of such parameters are by plotting and comparing with the empirical findings, as can be seen in Figures 4.5 and 4.4 where 2D distributions are kernel smoothed and plotted on top of one another.

\subsection{Evaluation}

Fitting the Brownian Motion based exploration of returns does not appear to be particularly successful, although this may merely be a matter of adjusting the model. Transforming the idea into a visual exploration and considering how best to move forward does however uncover some useful ideas. The Brownian Motion based estimation of parameters from first passage times does seem to be somewhat indicative of the first passage times; while this is difficult to test, this can be visually explored by comparing overlapping constrained distributions within $\tau \leq 60$ and $h \leq 25$ pips (1 pip price change for GBPUSD equals 0.0001).

Firstly, comparing the Naive trade distribution with the Euler estimation for Brownian Motion shows that to a great extent the empirical observations match closely with the 


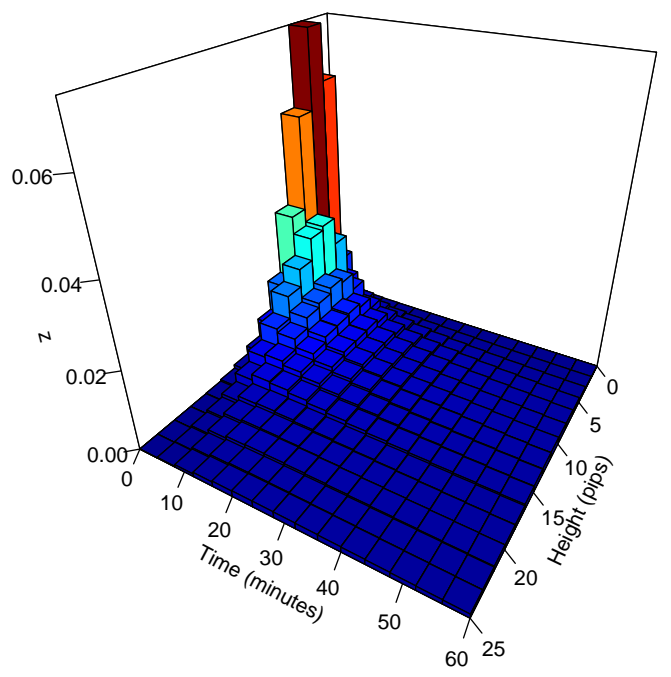

Figure 4.3: TBot Up Constrained Brownian Motion 2D First Passage Distribution $\mu=0$, $\sigma=0.173$

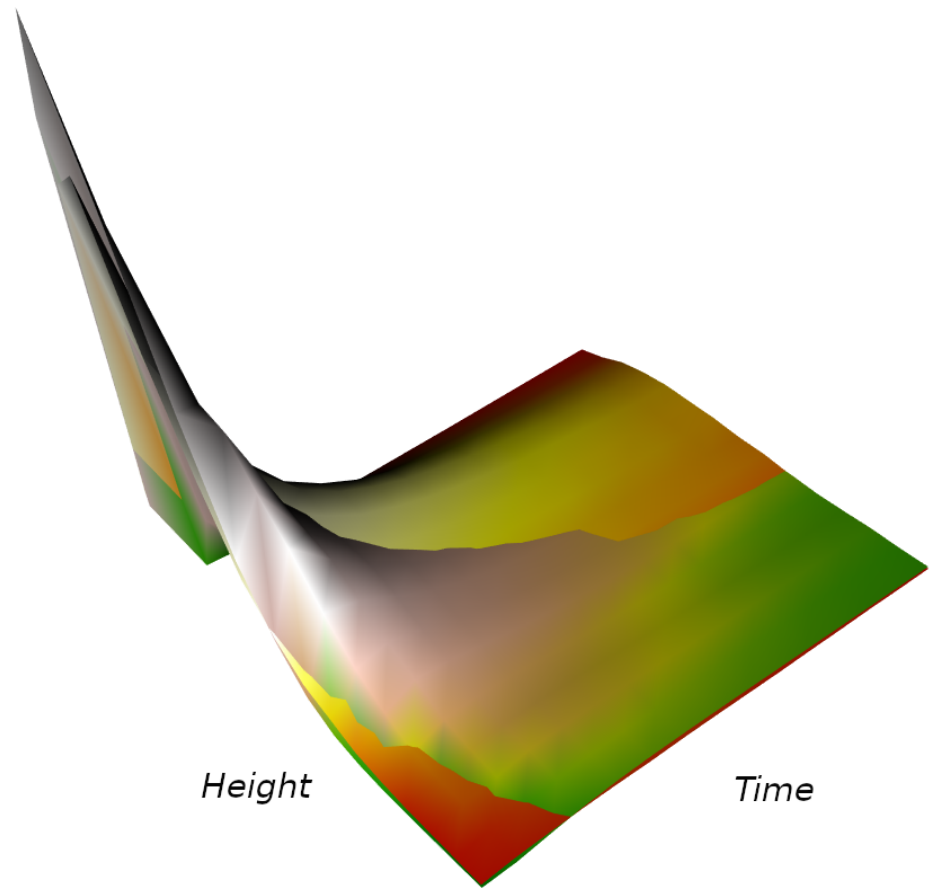

Figure 4.4: TBot Up Constrained Smoothed Naive (Heat) vs Brownian Motion (Terrain) $\sigma=0.173$

theoretical estimation as seen in Figure 4.4. This is awkward to see on the 2D distribution plot where the overlapping surfaces are reasonably close, but if this is considered carefully it can be seen that there is a slight difference in distribution with 
changes in $h$ which shows as the large green zone of colour. This slight difference in first passage time distribution seems quite reasonable, seemingly indicating some potential small structural difference which depends on the $h$ based change in price measured against.

These surfaces are sufficiently close that this corroborates well with the estimations as seen in Table 4.2 (when Brownian Motion parameters are used in the 2D plot, the surfaces of all types of Naive trades appear reasonably close). In the absence of more definitive testing, this shows that the estimation methods reasonably match with empirical results, enough so to roughly validate that these results are comparable.

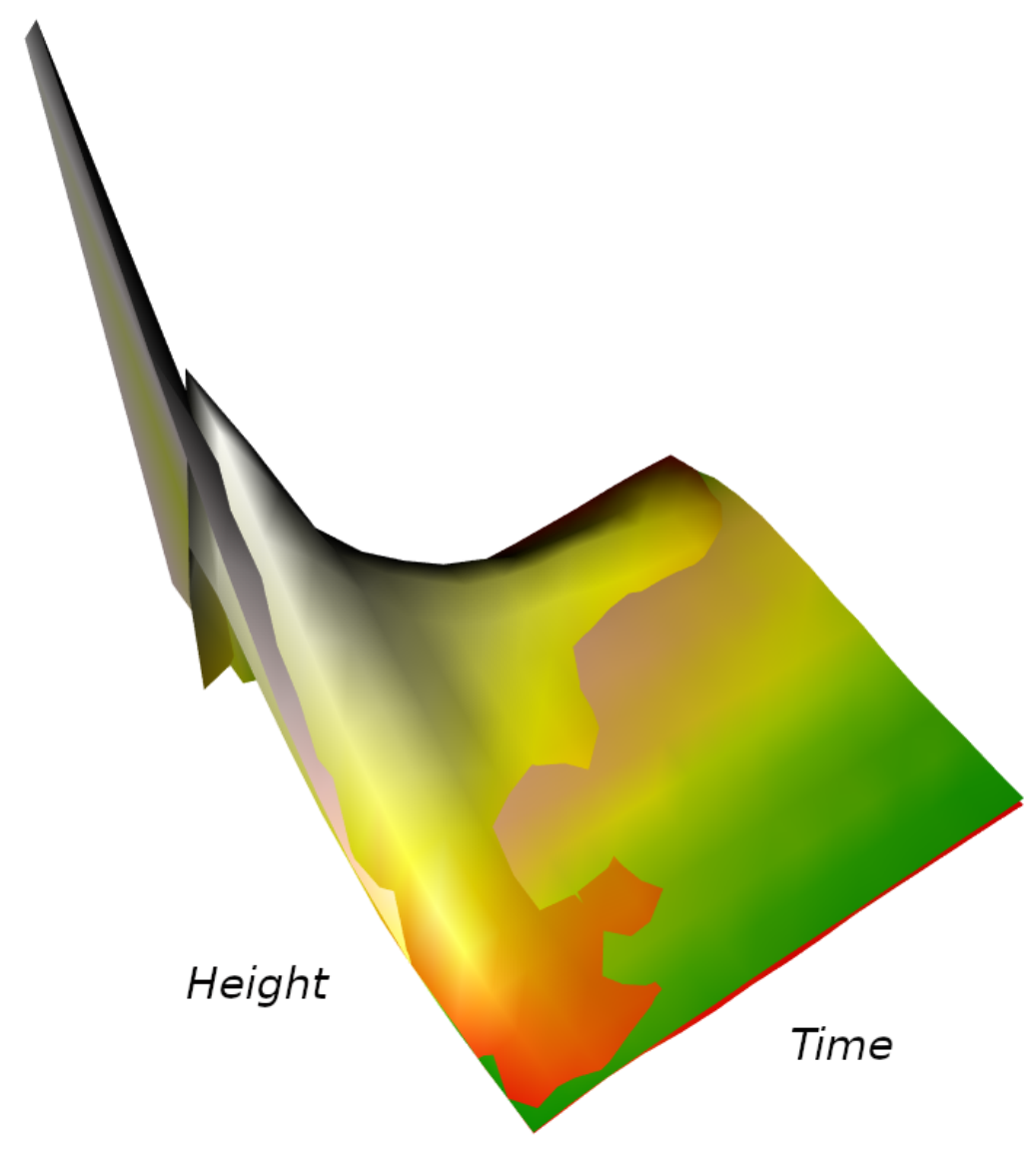

Figure 4.5: Constrained Smoothed Naive (Terrain) vs Triangle (Heat)

However the Triangle results are not similar, as can be seen with Naive and Triangle cases in Figure 4.5. (The comparison with Brownian Motion is not shown as this is very close to the Naive case.) This shows a disparity between low and high $h$ values where the low $h$ distribution section curves show Naive trade arrival times are comparatively shorter; and high $h$ distribution section curves show that Triangle base arrival times are comparatively shorter - noting that the $h$ sections are comparable since the masses in each $h$ based Triangle and Naive sections have been chosen to be the same. The higher $h$ values happen to correspond to price changes that would be more worthwhile trades, since there 
is a higher possibility on average that winning trades will overcome transaction costs. By trialling various Brownian Motion parameter values individually by $h$ section it seems unlikely that a raw Brownian Motion based model will fit well, and there seems a strong possibility the structure of the price changes is time varying.

Deriving and testing Itô processes to fit based on first passage times is complex, time consuming and in this case problematic. The possibility that the structural model is both time and $h$ varying presents an extremely difficult scenario to derive solutions for. Additionally, the structure of each of the Naive and Triangle cases is likely different and hence hard to compare. Comparing these using a realistic test is also not clear at all; although if the structures are the same, bootstrapping parameter confidence intervals may work and there is the possibility of making further good use of effect size based statistics. Starting with a continuous time based model of returns requires the assumption of a structure, and this likely involves too strong a set of assumptions to initially apply well. Without realising, I may have been heavily influenced to extrapolate periodic returns to continuous time, as is commonly done in the literature.

However the fact that there appears a change in structure across $h$ and potentially across $\tau$ presents an interesting possibility that a model can be produced with changes in intensity based on various more statistically based arrival time models. Through an ordering of arrival times the intensity can be comparatively measured and the corresponding 'difficult to model' left edge of the arrival time distribution more easily accounted for. Ideally once better understood it may then be possible to move back to a structural model. Though in the end I do not apply any structural approach to the US Equity results due to the complexities involved applying such an approach across multiple time series. 


\section{Chapter 5}

\section{Intensity Model}

For the Intensity approach, results of events are in terms of wins or losses of $\delta=1$ or 0 and arrival time $\tau$; where they are ordered by arrival time to form a non-homogeneous marked point Poisson process. The idea is to split the stopping time probabilities across wins and losses to create a mixture distribution, and model this using survival analysis. General information on survival analysis can be found in Kalbfleisch and Prentice (2002), though the approach used here is developed specifically to solve this mixture level based returns problem. On this basis, a mixed proportional survival model is derived into which any convenient base distribution for arrival intensity can be inserted. The Intensity model is represented in either a general form, without a base distribution; or specifically using the Generalized Extreme Value (GEV) distribution. With a specific model, maximum likelihood can be used to estimate the chosen distribution parameters to model short term momentum. Other alternative models are discussed in Section 8.1.

Estimation is obtained from data which consists of $h, \delta$ and $\tau$, where $h$ is predefined by the event height as described and $(\delta, \tau)$ pairs are results of those events in terms of win/loss and time taken to reach $h$ respectively. These are defined as

- $h$ half the absolute difference of the triangle price between extrema $E_{1}$ and $E_{2}$, generally referred to as height,

- $\tau$ Arrival times at the win $\tau^{+}$or loss $\tau^{-}$points where $\tau=\tau^{+} \wedge \tau^{-}$, where $\tau$ is the time difference between points $B$ to $C$,

- $\delta$ win or loss: with win $\delta=1$ when $r=1$ and loss $\delta=0$ when $r=-1$, which depends on the Triangle type as defined in Section 1.1.

The parameter $h$ is modified by dividing by volatility $h_{\sigma}=\frac{h}{\sigma}$ to normalise the data such that estimation is done over $\left(h_{\sigma}, \delta, \tau\right)$ result tuples. Generally $h$ may refer to $h$ or $h_{\sigma}$, as the precise definition of $h$ is immaterial in the derivations below.

In this case $h$ or $h_{\sigma}$ is contained in the filtration $\mathcal{F}_{t}$; however, $\mathcal{F}_{t}$ could incorporate covariates so is left in a general form until estimation. Maximum likelihood estimation 
requires several assumptions to be satisfied and practical adjustments as discussed in Section 5.2.

The estimation is done in slices of $h$ or $h_{\sigma}$ sections such that parameters are constant over certain $h$ based ranges. Originally the intention had been to fit the intensity model over the entire surface of $(h, \delta, \tau)$; but due to non-linearities it turned out to be impractical to obtain a good fit.

This Intensity model is inspired from credit risk models as found in McNeil et al. (2015), which often use extreme value theorem to deal with credit risk. The realisation was that by partly inverting the problem using price levels, rather than using periodic returns, the previous modelling of security risk is now a case modelling long term variation of price within the long right tail. The original risk of typical periodic return based models in the trails of the modelling distribution, are converted such that they are now contained in the left body of the Intensity distributions which are now able to be modelled by the events contained in right tail. This now becomes a problem of modelling the risk of nothing happening for longer periods of time (no large move in price) as opposed to modelling the risk of large changes in price.

\subsection{Base Intensity Model}

Consider the combined arrival time of wins and losses $\tau$ at close point $C$, starting at their break point $B$ where $t=0$; then order by arrival time such that they form a nonhomogeneous Poisson process defined on the real line as given by (5.1)

$$
\begin{aligned}
& \qquad \begin{aligned}
\mathbb{P}\left(X(t+s)-X(t)=n \mid \mathcal{F}_{t}\right) & =\frac{(\Lambda(t+s)-\Lambda(t))^{n} e^{-(\Lambda(t+s)-\Lambda(t))}}{n !} \\
\text { where } \Lambda(t) & =\int_{0}^{t} \lambda(u) d u \text { and } \Lambda(t+s)=\int_{0}^{t+s} \lambda(u) d u \\
\text { with intensity given by } \lambda(t) d t & \left.=d \Lambda(t)=\mathbb{P}\left(\tau<t+d t \mid \tau \geq t, \mathcal{F}_{t}\right)\right) \\
& =\frac{\mathbb{P}\left(t \leq \tau<t+d t \mid \mathcal{F}_{t}\right)}{\mathbb{P}\left(\tau \geq t \mid \mathcal{F}_{t}\right)} \\
& =\frac{f(t) d t}{1-F(t)} \in(0, \infty)
\end{aligned}
\end{aligned}
$$

I split the combined intensity of joint arrival time and win/loss probabilities under the Law of Total probability where 


$$
\begin{aligned}
\Lambda(t) & =\Lambda^{(+)}(t)+\Lambda^{(-)}(t) \\
d \Lambda^{(+)}(t) & =\mathbb{P}\left(\tau<t+d t, \delta=1 \mid \tau \geq t, \mathcal{F}_{t}\right) \\
d \Lambda^{(-)}(t) & =\mathbb{P}\left(\tau<t+d t, \delta=0 \mid \tau \geq t, \mathcal{F}_{t}\right) \\
\Longrightarrow d \Lambda(t) & =\mathbb{P}\left(\tau<t+d t \mid \tau \geq t, \mathcal{F}_{t}\right) \\
& =\frac{\mathbb{P}\left(t \leq \tau<t+d t \mid \mathcal{F}_{t}\right)}{\mathbb{P}\left(\tau \geq t \mid \mathcal{F}_{t}\right)} .
\end{aligned}
$$

Intensity can be expressed in terms of survival time as

$$
\begin{aligned}
S(t) & =\mathbb{P}\left(\tau \geq t \mid \mathcal{F}_{t}\right) \\
d \Lambda(t) & =\frac{-(S(t+d t)-S(t))}{S(t)} \\
& =\frac{-d S(t)}{S(t)}
\end{aligned}
$$

define $\Lambda(0)=0 ; \quad S(0)=1$

such that $d \Lambda(t)=-d \ln (S(t))$.

Survival time can then be expressed as

$$
\begin{aligned}
\int_{0}^{t} d \Lambda(t) & =\int_{0}^{t}-d \ln (S(t)) \\
\Lambda(t)-\Lambda(0) & =-(\ln (S(t))-\ln (S(0)) \\
\Lambda(t) & =-\ln (S(t)) \\
S(t) & =e^{-\Lambda(t)} .
\end{aligned}
$$

The first passage crossing time probabilities are pseudo independent since

$$
\begin{aligned}
\mathbb{P}\left(\tau>t \mid \mathcal{F}_{t}\right) & =e^{-\Lambda(t)} \\
\text { where }\{\tau>t\} & =\left\{\tau^{+}>t\right\} \cap\left\{\tau^{-}>t\right\} \\
\text { so } e^{-\Lambda(t)} & =\mathbb{P}\left(\tau^{+}>t, \tau^{-}>t \mid \mathcal{F}_{t}\right) \\
& =e^{-\Lambda^{(+)}(t)} e^{-\Lambda^{(-)}(t)} .
\end{aligned}
$$

It is assumed then that $(\tau, \delta)$ pairs are independent where $\mathcal{F}_{t}$ measurements are set at point $B$ and are constants from the perspective of waiting until point $C$. Therefore the likelihood contribution of $(\tau, \delta)$ conditional on $\mathcal{F}_{t}$ can be expressed as 


$$
\begin{aligned}
\mathcal{L}\left(t_{k}, t_{k}+d t\right) & =\mathbb{P}\left(t_{k} \leq \tau<t_{k}+d t, \delta \mid \mathcal{F}_{t}\right) \\
& =\mathbb{P}\left(\tau \geq t_{k}, \delta \mid \mathcal{F}_{t_{k}}\right) \mathbb{P}\left(\tau<t_{k}+d t, \delta \mid \tau \geq t_{k}, \mathcal{F}_{t_{k}}\right) \\
& =S\left(t_{k}\right)\left[d \Lambda^{(+)}\left(t_{k}\right)\right]^{\delta_{k}}\left[d \Lambda^{(-)}\left(t_{k}\right)\right]^{1-\delta_{k}} \\
\prod_{k} \mathcal{L}\left(t_{k}, t_{k}+d t\right) & =\prod_{k} S\left(t_{k}\right)\left[d \Lambda^{(+)}\left(t_{k}\right)\right]^{\delta_{k}}\left[d \Lambda^{(-)}\left(t_{k}\right)\right]^{1-\delta_{k}} \\
& =\prod_{k} S\left(t_{k}\right)\left[\frac{f^{(+)}\left(t_{k}\right) d t}{S\left(t_{k}\right)}\right]^{\delta_{k}}\left[\frac{f^{(-)}\left(t_{k}\right) d t}{S\left(t_{k}\right)}\right]^{1-\delta_{k}} \\
& =\prod_{k}\left[f^{(+)}\left(t_{k}\right) d t\right]^{\delta_{k}}\left[f^{(-)}\left(t_{k}\right) d t\right]^{1-\delta_{k}} \\
& =\prod_{k}\left[f^{(+)}\left(t_{k}\right)\right]^{\delta_{k}}\left[f^{(-)}\left(t_{k}\right)\right]^{1-\delta_{k}},
\end{aligned}
$$

where $d t$ may be ignored since it does not affect the estimation of the likelihood parameters. Under numerical estimation the equalities expressed in (5.6) are approximate, but for ease of expression I use the equals sign throughout the document.

Assuming that the measures $\mathbb{P}\left(t \leq \tau<t+d t, \delta=1 \mid \mathcal{F}_{t}\right)$ and $\mathbb{P}\left(t \leq \tau<t+d t, \delta=0 \mid \mathcal{F}_{t}\right)$ are absolutely continuous with respect to Lebesgue measure, then

$$
d \Lambda^{(+)}(t)=\frac{f^{(+)}(t) d t}{S(t)}, \quad d \Lambda^{(-)}(t)=\frac{f^{(-)}(t) d t}{S(t)} .
$$

However the densities consisting of $f^{(+)}(t)$ and $f^{(-)}(t)$ under the Law of Total probability must form a correct probability density such that distribution functions integrate to one where

$$
\begin{aligned}
f^{(+)}(t) d t & \left.=\mathbb{P}\left(t \leq \tau<t+d t, \delta=1 \mid \mathcal{F}_{t}\right)\right) \\
f^{(-)}(t) d t & \left.=\mathbb{P}\left(t \leq \tau<t+d t, \delta=0 \mid \mathcal{F}_{t}\right)\right) \\
f(t) d t & =\left(f^{(+)}(t)+f^{(-)}(t)\right) d t \\
\int_{0}^{\infty} f(t) d t & =1, \int_{0}^{\infty} f^{(+)}(t) d t \leq 1, \int_{0}^{\infty} f^{(-)}(t) d t \leq 1 .
\end{aligned}
$$

Hence $f(t)$ forms a mixture density consisting of $f^{(+)}$and $f^{(-)}$components, where functions can be inserted to model corresponding wins and losses. As an example consider the initial base motivating case where the functions $f^{( \pm)}$evolve exponentially to from a Poisson process such that 


$$
\begin{aligned}
f^{(+)}(t) & =p_{1} f_{1}(t)=p_{1} \lambda_{1} e^{-\lambda_{1} t} \\
f^{(-)}(t) & =p_{2} f_{2}(t)=p_{2} \lambda_{2} e^{-\lambda_{2} t} \\
\int_{0}^{\infty} p_{1} \lambda_{1} e^{-\lambda_{1} t} d t & =p_{1} \\
\int_{0}^{\infty} p_{2} \lambda_{2} e^{-\lambda_{2} t} d t & =p_{2}
\end{aligned}
$$

under restriction $p_{1}+p_{2}=1$

$$
\text { where } F^{(+)}+F^{(-)}=F \text {. }
$$

To differentiate between these functions I refer to $f^{(+)}$and $f^{(-)}$components of the mixture distribution as sub-densities; and $F^{(+)}$and $F^{(-)}$components as sub-distributions; where summed they form a density $f$ or distribution $F$ respectively. I use numerical subscripts to refer to proportionate component densities and distributions. Using this notation, for the Intensity model any inserted distributions must conform to (5.8) such that the density functions can be split by proportion as demonstrated in (5.9).

\subsection{Estimation Considerations}

Originally estimation had been over the $(h, \tau)$ surface but this had various convergence problems, and after some experimentation a fit using three $h_{\sigma}$ slices was used, as demonstrated in the trial discussed in Appendix C. These three slices represent three zones of high to low volatility relative to change in price $h$.

Actual estimation of these slices results in a formula which is closer to (5.10), but this is awkward to express every time maximum likelihood is mentioned. Expressed without indices this can be seen as

$$
\begin{aligned}
\prod \mathcal{L}\left(t_{1 / 2}, t_{1 / 2}+1\right) & =\prod\left[f^{(+)}\left(t_{1 / 2}\right)\right]^{\delta}\left[f^{(-)}\left(t_{1 / 2}\right)\right]^{1-\delta} & & \text { estimated at } \frac{1}{2} t \text { points } \\
& =\prod\left[p_{1} f_{1}\left(t_{1 / 2}\right)\right]^{\delta}\left[p_{2} f_{2}\left(t_{1 / 2}\right)\right]^{1-\delta} & & \text { proportionate densities } \\
& =\prod\left[p f_{1}\left(t_{1 / 2}\right)\right]^{\delta}\left[(1-p) f_{2}\left(t_{1 / 2}\right)\right]^{1-\delta} & & \text { constrained proportion } p .
\end{aligned}
$$

This is adjusted by measuring the arrival histogram at the mid point within measuring periods along the $x$ axis form a more accurate estimate. This is done because measurements are not done on a continuous basis but are over single units measured at the right edge of the period. For example if one simulated trade stops on the 4th day's measurement then that trade has 'stopped' at some point within that day, say at for 
example 3.6 days. To offset this I subtract $1 / 2$ from each day for estimation purposes, which is a rough but serviceable approximation. In essence the data is in a discrete form but it is pragmatic to estimate using the continuous form by making small adjustments (ironically converting back to a binomial form to test the goodness of fit). As it happens this is an important consideration since a large part of the mass of the sub-distribution usually occurs within the first few periods and so this results in better approximation.

Since $1=p_{1}+p_{2}$, only the variable $p_{1}$ is required in estimation, as seen in the last line of (5.10). For simplicity all results only report $p_{1}=p$, which is the probability of winning a trade in the direction indicated by the triangle.

For many trial distributions of $f^{(+)}$and $f^{(-)}$, including the Generalized Extreme Value distribution, taking the $\log$ and subsequent derivatives is unsolvable. So generally a numerical approach is taken using the $\mathrm{R}$ optim function to find the maximum likelihood. Due to numerous exponentials this requires some manipulation and often constraints to find a solution. In some cases this requires a choice of an approximate best fit since toward an edge of the manifold optimisation results in an error or has an undefined value. Thus this is often time consuming to visually check that the fit is correct and occasionally requires some manual selection.

\subsection{Generalized Extreme Value Base Model}

Primarily I use Generalized Extreme Value distribution (GEV) as the base model by truncating and fitting proportionate mixed distributions representing win and loss arrival times. Truncated GEV allows a good fit as it allows adjustment of the distribution mode (head peak position), head thickness and fatness of the right tail. This gives the flexibility to fit against various long tailed waiting times such that goodness of fit is generally high against in-sample fitted data.

The GEV distribution is characterized by location $\mu$, scale $\sigma$ and shape $\xi$ for density $f$ and distribution function $G$ as

$$
\begin{aligned}
s=\frac{t-\mu}{\sigma} \quad \text { such that } & \\
g(s ; \sigma, \xi) & = \begin{cases}\frac{1}{\sigma}(1+\xi s)^{\frac{-1}{\xi}-1} e^{-(1+\xi s)^{\frac{-1}{\xi}}}, & \xi \neq 0 \\
\frac{1}{\sigma} e^{-s} e^{-e^{-s}}, & \xi=0\end{cases} \\
G(s ; \xi) & = \begin{cases}e^{-(1+\xi s)^{\frac{-1}{\xi}},} & \xi \neq 0 \\
e^{-e^{-s}}, & \xi=0 .\end{cases}
\end{aligned}
$$

However, support for GEV may include negative values, depending on values of $\xi$ : when $\xi>0, \quad t \in\left[\mu-\frac{\sigma}{\xi},+\infty\right)$. So the distribution must be truncated to produce support 
$[0,+\infty)$ for truncated density $g_{T}$ and distribution $G_{T}$

$$
\begin{aligned}
g_{T}(t) & =\frac{g(t)}{1-G(a)} \\
G_{T}(t) & =\frac{G(t)-G(a)}{1-G(a)}
\end{aligned}
$$

where $a=0$ as the point of left truncation. I mix distributions similarly to example 5.9 and multiply the above by $p$ obtain the Intensity model

$$
\begin{aligned}
f^{(+)}(t) & =p_{1} g_{T 1}(t) \\
f^{(-)}(t) & =p_{2} g_{T 2}(t) \\
\int_{0}^{\infty} p_{1} g_{T 1}(t) d t & =p_{1} \\
\int_{0}^{\infty} p_{2} g_{T 2}(t) d t & =p_{2} \\
\text { under the restriction } \quad p_{1}+p_{2} & =1 \\
\text { such that } \quad F^{(+)}+F^{(-)} & =F,
\end{aligned}
$$

where under truncation the lower bound of the integrals are 0 . As indicated earlier, subdensities $f^{(+)}$and $f^{(-)}$together form density $f$; and sub-distributions $F^{(+)}$and $F^{(-)}$ together form distribution $F$. The likelihood function for Intensity GEV densities given in (5.13) is then

$$
\begin{aligned}
\mathcal{L}(t, t+d t) & =\mathbb{P}\left(t \leq \tau<t+d t, \delta \mid \mathcal{F}_{t}\right) \\
& =\left[f^{(+)}(t)\right]^{\delta}\left[f^{(-)}(t)\right]^{1-\delta} \quad \text { from }(5.6) \\
& =\left[\frac{\frac{p_{1}}{\sigma_{1}}\left(1+\xi_{1} s_{1}\right)^{\frac{-1}{\xi_{1}}-1} e^{-\left(1+\xi_{1} s_{1}\right)^{\frac{-1}{\xi_{1}}}}}{1-e^{-\left(1+\xi_{1} s_{1}\right)^{\frac{-1}{\xi_{1}}}}}\right]^{\delta}\left[\frac{\frac{p_{2}}{\sigma_{2}}\left(1+\xi_{2} s_{2}\right)^{\frac{-1}{\xi_{2}}-1} e^{-\left(1+\xi_{2} s_{2}\right)^{\frac{-1}{\xi_{2}}}}}{1-e^{-\left(1+\xi_{2} s_{2}\right)^{\frac{-1}{\xi_{2}}}}}\right]^{1-\delta},
\end{aligned}
$$

where $\xi_{1} \neq 0, \xi_{2} \neq 0$. Cumulative probabilities in terms of GEV for sub and full distributions are then 


$$
\begin{aligned}
& \mathbb{P}\left(\tau<t, \delta=1 \mid \mathcal{F}_{t}\right)=p_{1} G_{T 1}(t)=F^{(+)}(t) \\
& \text { Since } p_{1}=\mathbb{P}\left(\delta=1 \mid \mathcal{F}_{t}\right) \\
& \mathbb{P}\left(\tau<t \mid \delta=1, \mathcal{F}_{t}\right)=G_{T 1}(t) \\
& =\frac{e^{-\left(1+\xi_{1} \frac{t-\mu_{1}}{\sigma_{1}}\right)^{\frac{-1}{\xi_{1}}}}-e^{-\left(1-\frac{\xi_{1} \mu_{1}}{\sigma_{1}}\right)^{\frac{-1}{\xi_{1}}}}}{1-e^{-\left(1-\frac{\xi_{1} \mu_{1}}{\sigma_{1}}\right)^{\frac{-1}{\xi_{1}}}}} ; \quad \xi_{1} \neq 0
\end{aligned}
$$

similarly for $F^{(-)}$

$$
\begin{aligned}
\mathbb{P}\left(\tau<t, \delta=0 \mid \mathcal{F}_{t}\right) & =p_{2} G_{T 2}(t)=F^{(-)}(t) \\
\text { since } p_{2} & =\mathbb{P}\left(\delta=0 \mid \mathcal{F}_{t}\right) \\
\mathbb{P}\left(\tau<t \mid \delta=0, \mathcal{F}_{t}\right) & =G_{T 2}(t) \\
& =\frac{e^{-\left(1+\xi_{2} \frac{t-\mu_{2}}{\sigma_{2}}\right)^{\frac{-1}{\xi_{2}}}}-e^{-\left(1-\frac{\xi_{2} \mu_{2}}{\sigma_{2}}\right) \frac{-1}{\xi_{2}}}}{1-e^{-\left(1-\frac{\xi_{2} \mu_{2}}{\sigma_{2}}\right)^{\frac{-1}{\xi_{2}}}}} ; \quad \xi_{2} \neq 0 .
\end{aligned}
$$

For brevity results are expressed using these distribution and parameter symbols as given above. The following goodness of fit tests use these cumulative probabilities. Also these are used to express results in terms of probability of arrival within time $t$ towards expressing short term momentum. Note: at no point are estimated $\xi_{1}=0$ or $\xi_{2}=0$ so reference to conditions for $\xi$ for GEV distributions are now dropped.

\subsubsection{Parameter Estimation}

Using the considerations for estimation as demonstrated in (5.10), the full likelihood estimation equation can be seen as (5.16). Table 5.1 then shows the corresponding parameter estimates using $h_{\sigma}$ sections, where $t$ is measured in minutes and $h_{\sigma}$ are in units of $\frac{h \text { GBPUSD price }}{\text { minutes }}$. An example of this can be seen visually in Figure 5.1 which shows PDF and CDF comparisons for the model versus the empirical data for the particular range $h_{\sigma} \in[0.0035,007)$; such plots I refer to as 'profile plots'. This shows what appears to be a good fit of the model to the data for all cases.

$\mathcal{L}(t, t+d t)=\left[\frac{\frac{p}{\sigma_{1}}\left(1+\xi_{1} \frac{t-\mu_{1}}{\sigma_{1}}\right)^{\frac{-1}{\xi_{1}}-1} e^{-\left(1+\xi_{1} \frac{t-\mu_{1}}{\sigma_{1}}\right)^{\frac{-1}{\xi_{1}}}}}{1-e^{-\left(1+\xi_{1} \frac{t-\mu_{1}}{\sigma_{1}}\right)^{\frac{-1}{\xi_{1}}}}}\right]^{\delta}\left[\frac{\frac{(1-p)}{\sigma_{2}}\left(1+\xi_{2} \frac{t-\mu_{2}}{\sigma_{2}}\right)^{\frac{-1}{\xi_{2}}-1} e^{-\left(1+\xi_{2} \frac{t-\mu_{2}}{\sigma_{2}}\right)^{\frac{-1}{\xi_{2}}}}}{1-e^{-\left(1+\xi_{2} \frac{t-\mu_{2}}{\sigma_{2}}\right)^{\frac{-1}{\xi_{2}}}}}\right]^{1-\delta}$ 
Table 5.1: GBPUSD $h_{\sigma}$ Intensity Model Parameter Estimates

\begin{tabular}{lllllllllll}
\hline & & \multicolumn{3}{c}{ Win Distribution } & & \multicolumn{3}{c}{ Loss Distribution } \\
Type & $h_{\sigma}$ & $\mu_{1}$ & $\sigma_{1}$ & $\xi_{1}$ & & $\mu_{2}$ & $\sigma_{2}$ & $\xi_{2}$ & $p$ \\
\hline TTop Up & {$[0.0035)$} & 2.52 & 6.45 & 0.519 & & 5.3 & 7.48 & 0.489 & 0.513 \\
TTop Up & {$[.0035 .007)$} & 8.6 & 10.1 & 0.786 & & 13.1 & 13.9 & 0.661 & 0.51 \\
TTop Up & {$[.007 \infty)$} & 15 & 26.7 & 0.99 & & 20 & 39.1 & 0.836 & 0.507 \\
TTop Down & {$[0.0035)$} & 0.5 & 6.52 & 0.49 & & 3 & 7.72 & 0.491 & 0.534 \\
TTop Down & {$[.0035 .007)$} & 9.57 & 11.2 & 0.764 & & 10 & 14.8 & 0.6 & 0.523 \\
TTop Down & {$[.007 \infty)$} & 10 & 28.4 & 0.849 & & 28.4 & 34 & 0.99 & 0.539 \\
TBot Down & {$[0.0035)$} & 3.29 & 5.76 & 0.527 & & 5.65 & 7.45 & 0.536 & 0.517 \\
TBot Down & {$[.0035 .007)$} & 7.9 & 10.4 & 0.793 & & 10 & 14.4 & 0.596 & 0.514 \\
TBot Down & {$[.007 \infty)$} & 15 & 26.9 & 0.99 & & 30.2 & 35.7 & 0.933 & 0.505 \\
TBot Up & {$[0.0035)$} & 2.57 & 6.19 & 0.628 & & 0.5 & 8.25 & 0.4 & 0.523 \\
TBot Up & {$[.0035 .007)$} & 9.15 & 12.1 & 0.732 & & 11.7 & 13.2 & 0.701 & 0.519 \\
TBot Up & {$[.007 \infty)$} & 15 & 27.4 & 0.967 & & 16.8 & 38.9 & 0.835 & 0.527 \\
\hline
\end{tabular}

Win PDF

Loss PDF
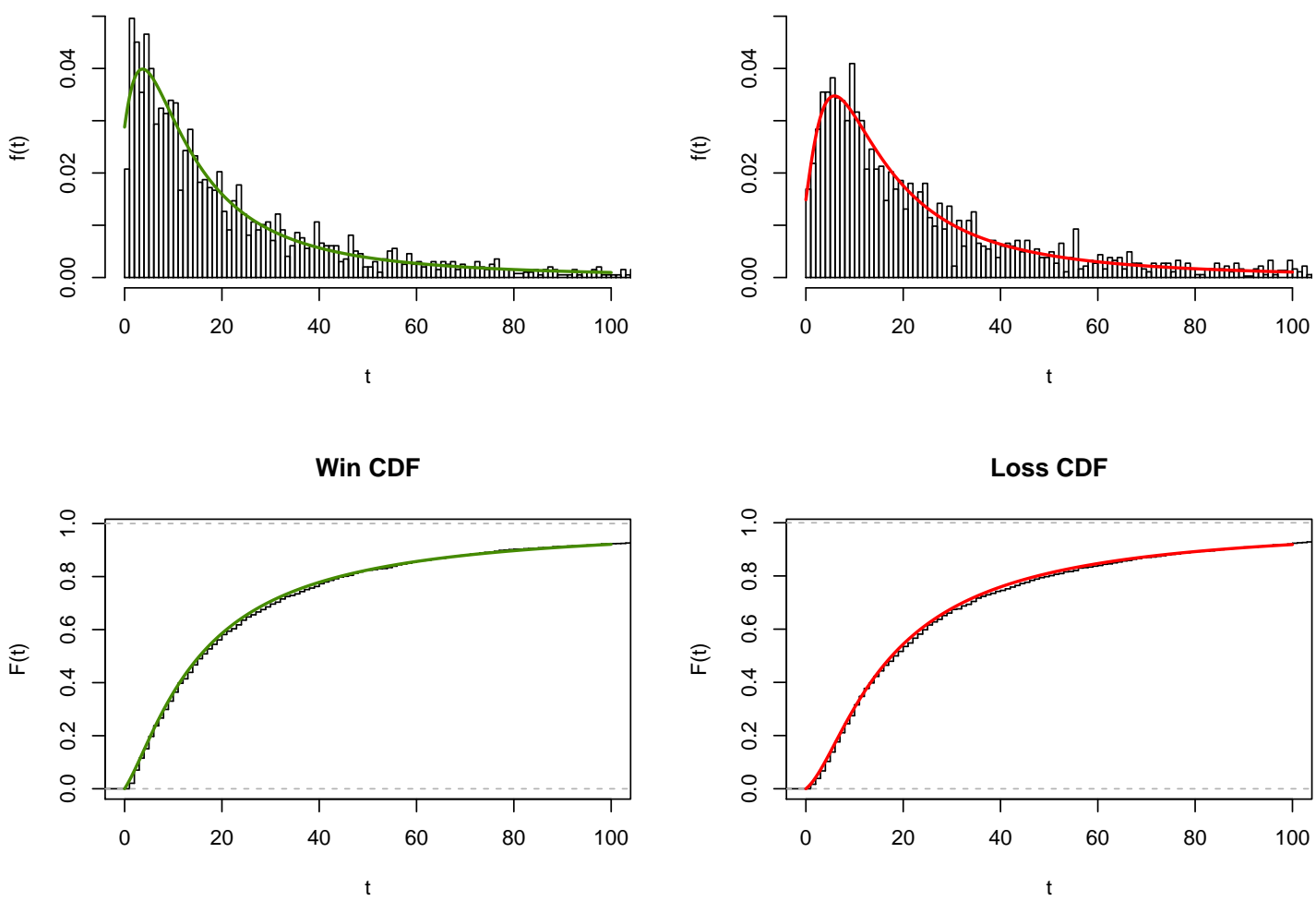

Figure 5.1: TBot Up Intensity Distribution Profile $h_{\sigma} \in[0.0035,007)$

\subsection{Goodness of Fit}

Goodness of fit testing for the model requires checks across: in-sample conditional triangle fit returns; Naive in-sample simulated returns and forward out-of-sample triangle returns. In-sample returns are done to confirm that the model shows the hybrid 
arrival time and win distribution fits well; however, of greater importance is the out-of-sample forward tests which evaluate how the model holds towards prediction of short term momentum. The fitted model is tested against the Naive data to check if there is a difference. The test used is the Cumulated Deviations test from actuarial science which supposes that the distribution of arrival times is Poisson or Binomial.

The reported p-value evaluates under the null hypothesis whether first passage times given by the data are equivalent to the distribution (or sub-distributions) given by the estimated intensity model. The p-value is often high as it is a confirmatory test, the measure of which is being used to evaluate the goodness of fit. The intention of this is to evaluate in-sample results and then measure predictability of short term momentum.

\subsubsection{Cumulative Deviations Test Construction}

For testing purposes since the data is recorded in discrete time and modelling is done in continuous time, it is necessary to assume a non-homogeneous Binomial process to approximate the data. This is done by assuming that for large sample size the continuous Poisson arrival times are approximated by discrete binomial intensity $\lambda_{t}^{b} d t=\mathbb{P}(\tau \leq t+$ $d t \mid \tau>t) \in[0,1]$, where in each individual period $\lambda_{t}^{b}$ remains constant, though it may differ from period to period.

The Cumulative Deviations Test is a direct extension of Pearson's Chi squared test specific to Poisson and Binomial processes. Treating the results as Binomial arrival times the two test statistics $Z_{t}$ for wins and losses can be formed by considering cumulative probabilities of single units of time as

$$
\begin{aligned}
& Z_{t}=\frac{d_{t}^{s}-q_{t}^{s} N_{t}}{\sqrt{q_{t}^{s}\left(1-q_{t}^{s}\right) N_{t}}} \sim \mathcal{N}(0,1) \quad \text { for sub-densities denoted } s \\
& q_{t}^{s}= F^{( \pm)}(t)-F^{( \pm)}(t-1) \\
& t \in[1, n] ; \quad n:=\max (\text { arrival time }) \\
& N_{t}:=\text { count of all win and loss arrivals } \\
& d_{t}^{s}:=\text { count of only win or loss arrivals in }(t-1, t], \\
& \\
& Z_{t}=\frac{d_{t}-q_{t} N_{t}}{\sqrt{q_{t} N_{t}}} \sim \mathcal{N}(0,1) \quad \text { for complete densities } \\
& q_{t}=F(t)-F(t-1) \\
& t \in[1, n] \quad n:=\text { max (arrival time) } \\
& N_{t}:=\text { count of all arrivals that pertain to that density } \\
& d_{t}:=\text { count of arrivals in }(t-1, t],
\end{aligned}
$$

under assumption 


$$
\begin{aligned}
\sum_{t \in I} Z_{t} & \sim \mathcal{N}(0,|I|) \\
& \sim \mathcal{N}(0,1)
\end{aligned}
$$

where $I$ is the range of individual groups whose size is defined by $|I|$, which in this study this is 1 in all cases ( 1 minute or 1 day for US equities). Normality follows from the linearity of the Normal distribution.

Hence we can then construct the Cumulative Deviations test for these two cases as

$$
\begin{gathered}
\frac{\sum_{t \in I}\left(d_{t}^{s}-q_{t}^{s} N_{t}\right)}{\sqrt{\sum_{t \in I} q_{t}^{s}\left(1-q_{t}^{s}\right) N_{t}}} \sim \mathcal{N}(0,1) \quad \text { for sub-densities denoted } s \\
\frac{\sum_{t \in I}\left(d_{t}-q_{t} N_{t}\right)}{\sqrt{\sum_{t \in I} q_{t} N_{t}}} \sim \mathcal{N}(0,1) \text { for complete densities. }
\end{gathered}
$$

These use survival analysis models where $q$ is usually the mortality rate and $d$ is the observed number of deaths.

\subsubsection{Goodness of Fit Test Strategy}

Goodness of fit testing is applied using the sub-densities with and without their proportionate component across three dimensions for a total combination of 12 test types for each triangle type. The overall method is described here and the results given in their relevant section along with diagrams to explain further.

The Cumulative Deviations test uses the following conversions from the Poisson case with $d t=1$ for each period to test the following:

a. $\mathbb{P}\left(t \leq \tau<t+d t, \delta=1 \mid \mathcal{F}_{t}\right)$ joint probability fit of winning and arriving within each period (sub-distribution).

b. $\mathbb{P}\left(t \leq \tau<t+d t \mid \delta=1, \mathcal{F}_{t}\right)$ probability fit of arriving within each period conditional on winning (full distribution).

c. $\mathbb{P}\left(t \leq \tau<t+d t<t, \delta=0 \mid \mathcal{F}_{t}\right)$ joint probability of losing and arriving within each period (sub-distribution).

d. $\mathbb{P}\left(t \leq \tau<t+d t<t \mid \delta=0, \mathcal{F}_{t}\right)$ probability fit of arriving within each period conditional on losing (full distribution).

Each of these tests is done across

1. In-sample conditional triangle fit returns: test fit of in-sample data used to train maximum likelihood estimation (generally the p-value is close to 1 indicating a good fit).

2. Naive in-sample simulated returns: test the Naive data in the same category as 
the 'in-sample conditional Triangle fitted returns' case. This tests whether returns come from the same distribution as that fitted to the Triangle data.

3. Forward out-of-sample Triangle returns: tests the fit of the current period Triangle GEV model to ascertain whether the distribution of results remains valid forward in time.

Using the trial GBPUSD data for the in-sample sub-distribution tests the results can be seen in Table 5.2. This shows that the data fits the model well and that relevant distribution on the profile plot corroborates that this is clearly the case as seen in Figure 5.1 for TBot $\mathrm{Up} h_{\sigma} \in[0.0035,0.007)$ with $\mathrm{p}$-values $\approx 0.6$.

Table 5.2: GBPUSD $h_{\sigma}$ Triangle Intensity Model In Sample Tests

\begin{tabular}{|c|c|c|c|}
\hline \multirow[b]{2}{*}{ Type } & \multirow[b]{2}{*}{$h_{\sigma}$} & \multirow{2}{*}{$\begin{array}{l}\text { Win Distribution } \\
p G_{T 1}(t)\end{array}$} & \multirow{2}{*}{$\begin{array}{l}\text { Loss Distribution } \\
(1-p) G_{T 2}(t)\end{array}$} \\
\hline & & & \\
\hline TTop Up & [0.0035) & 0.667 & 0.658 \\
\hline TTop Up & [.0035 .007$)$ & 0.98 & 0.966 \\
\hline TTop Up & {$[.007 \infty)$} & 0.39 & 0.984 \\
\hline TTop Down & [0.0035) & 0.775 & 0.76 \\
\hline TTop Down & [.0035 .007) & 0.26 & 0.263 \\
\hline TTop Down & {$[.007 \infty)$} & 0.5 & 0.125 \\
\hline TBot Down & [0 .0035) & 0.493 & 0.478 \\
\hline TBot Down & [.0035 .007) & 0.764 & 0.71 \\
\hline TBot Down & {$[.007 \infty)$} & 0.151 & 0.597 \\
\hline TBot Up & [0 .0035) & 0.951 & 0.946 \\
\hline TBot Up & [.0035 .007) & 0.565 & 0.591 \\
\hline TBot Up & {$[.007 \infty)$} & 0.779 & 0.296 \\
\hline
\end{tabular}

\subsection{Evaluation}

The Intensity GEV model fits well across $h_{\sigma}$ sections, provides indication of the win and loss effect size and is straight forward to estimate. Also, a visually intuitive representation of arrival density can be produced which gives a comparative indication of the return distribution. The issues are that the parameters are unintuitive and the tests involve the use of $\mathrm{p}$-values.

The $\mathrm{p}$-value issue can be mitigated by repeatedly confirming tests on separate data sections and testing how the model holds on forward out-of-sample data. Also the p-value based fit is confirmatory and the model fit particularly strong such that there is no marginal rejection of a null hypothesis. I considered providing bootstrapped confidence intervals for model parameters but since there is a dependence between them they are not comparable to other fitted GEV models; model estimation can also be unstable. Overall providing effect size based depth is problematic using this model.

The six parameters for the GEV based sub CDFs are difficult to intuitively understand. 
In contrast $\mu$ and $\sigma$ for the normal distribution show clear central tendency and spread characteristics - an advantage of using log normal periodic returns. Additionally the Intensity Model compromises by excluding Triangles which do not reach $\pm h$, though there are a very small number of these, fewer than $0.1 \%$ of the total. The Intensity Model based on GEV is a particularly flexible distribution.

Typically statisticians will state that it is assumed the data is generated by the model, but I do not do this as this as this is an unreasonably strong statement for the model fit. The Intensity GEV model is exceptionally flexible and happens to fit the data well; however the first few periods of the left tail are unpredictable and difficult to model. Instead I use the fact that the model fits well over the right tail; and the left tail can be predicted by subtraction since $p_{\text {left }}=1-p_{\text {right }}$. This can be seen in Figure 5.1 where the first five minutes do not fit well for the win distribution. It turns out to be extremely difficult to fit these first few periods; however by ordering and modelling the entire distribution of stopping times, by inversion the first few periods are roughly able to be modelled. This method partly overcomes the left tail modelling problems of the Structural model, but is certainly not perfect.

Also it is worth being careful to note statistical testing involving p-values can be sensitive to large sample size causing misleading test results, especially with measurement inaccuracies (Schwab et al., 2011). So, I consider the p-values as purely a measure of fit and not a strict hypothesis test, since I do not strictly know how reliable the measure is, see Section 2.3. I then repeat these goodness of test fits to cumulatively infer understanding, rather than rely on an unknown level of accuracy.

Considering these issues of the Intensity model motivates using a non model based measure of momentum within a fixed time period as a confirmatory approach. This is covered by the Effect Size Heuristics Chapter 6. I discuss these issues further in the Discussion Chapter 8 amongst other related material and results which help to provide more insights into the issues. 


\section{Chapter 6}

\section{Effect Size Heuristics}

Effect size heuristics are an increasingly accepted core statistical method used to validate scientific claims, often used to replace statistical methods based on p-values. It seems reasonable to apply these methods to financial returns. This chapter develops effect size heuristics to explore the difference between Triangle and Naive models, based on bootstrapped empirical confidence intervals. The idea is that if the confidence intervals of return based measurements for Triangles and Naive trades are far apart then there may be a significant effect. No trial data is used to develop these methods, as they can be directly applied without iterative development.

For the effect size heuristics, a lock-in time of 10 days is set, such that any trade which has not reached $\pm h$ is closed at the closing price on day 10; otherwise the trade will have exited before this. This is done for all Triangle and Naive trades. For Triangle trades the existing discovered Triangles are used and returns re-calculated based on the 10 day limit. For Naive trades, first passage sampling is redone with the 10 day limit.

To recap the Naive method in this context, for each Triangle the value of $h$ is recorded and 10 sample points are chosen at random from the original stock time series, each denoted point $B$. For each of these randomly chosen points, the change in time $\tau$ is recoded by walking forward over segment $B C$ to $\pm h$; however in this chapter the change in time is limited to a maximum of 10 days. This is done such that if $\pm h$ is not reached by day 10 , partial $\pm h$ values are recorded to reflect the change in price reached on day 10 . The objective is to create a comparative strategy without the potential timing advantage of Triangles as discussed in detail within Section 3.3.

Using standard effect size statistics recommendations, a 95\% confidence interval is used for all measurements to identify the significance of Triangle and Naive return differences. One issue is how to compare leveraged returns given differing mean $\mu_{r_{l}}$ and standard deviation $\sigma_{r_{l}}$ values (as defined in (4.1)). Conveniently Cohen's d as seen in Cumming (2012) provides a standard difference in mean effect size normalised by pooled variance 


$$
\begin{aligned}
d & =\frac{\mu_{1}-\mu_{2}}{s} \\
s & =\sqrt{\frac{\left(n_{1}-1\right) s_{1}^{2}+\left(n_{2}-1\right) s_{2}^{2}}{n_{1}+n_{2}-2}} \\
s_{j}^{2} & =\frac{1}{n_{j}-1} \sum_{i=1}^{n_{j}}\left(x_{j, i}-\mu_{j}\right)^{2} \quad \text { for } j=1,2 ;
\end{aligned}
$$

where $\mu_{1}, s_{1}$ and $\mu_{2}, s_{2}$ refer to Triangle and Naive trade leveraged return and standard deviations respectively. Cohen's $d$ can then be used to provide a single standardised numerical value to measure the leveraged return difference. Since the distribution of Cohen's d is non-Gaussian and unknown I leave it in this form.

Due to the asymmetric nature of the measurements and unknown empirical distributions, percentile bootstrapped confidence intervals for the parameters of interest are produced. These are calculated on the leveraged return based measurements as given by $\mathrm{R}$ code listings in Appendix E. Using a bootstrapped percentile based confidence interval has the advantage of making very few assumptions about how the data is distributed and thereby avoids modelling error.

Bootstrapping is where sampling with replacement is used to estimate population parameters. Sampling is done repeatedly from the original sample with replacement to produce $N$ samples of size $n$, producing estimates of the parameter $\hat{\theta}_{1} . . \hat{\theta}_{N}$ with mean estimate $\hat{\theta}_{B}$. Denoting the $\frac{\alpha}{2}$ and $1-\frac{\alpha}{2}$ percentiles by $\epsilon_{L}$ and $\epsilon_{H}$ respectively I have

$$
\mathbb{P}\left(\epsilon_{L}<\hat{\theta}_{B}-\hat{\theta}<\epsilon_{H}\right)=1-\alpha
$$

so that the probability of an error less than $\epsilon_{L}$ is $\frac{\alpha}{2}$, and the probability of an error greater than $\epsilon_{H}$ is $\frac{\alpha}{2}$. Assuming the distribution of $\hat{\theta}$ about the population parameter $\theta$ from sampling the original sample is the same as would be obtained from the bootstrapped population, the following holds

$$
\mathbb{P}\left(\epsilon_{L}<\hat{\theta}-\theta<\epsilon_{H}\right)=1-\alpha
$$

In practice this procedure is simply a case of calculating the percentiles of the sampled bootstrap estimates. This theory is justified and detailed further in Manly (2007).

While the empirical percentile confidence interval is less accurate than the standard bootstrap, Buckland (1984) suggests 10,000 samples will produce a confidence interval of $95 \% \pm .1 \%$ with probability 0.95 . This is easily accurate enough for these measurements. So for the results $N=10000$ samples of size $n$ are taken, where $n$ is the size of the original sample. 
I calculate confidence intervals using this method for mean and standard deviation across: stopping time, bet return and leveraged return. For leveraged return Cohen's d is also bootstrapped to give a $Z$ based normalised return. These results can be seen in Table 7.6 for Triangles and Table 7.7 for Naive trades. As stated the implementation for each of these can be seen in Appendix E. 


\section{Chapter 7}

\section{US Equity Results}

The US equity based results for the Intensity model and Effect Size Heuristics are contained within this chapter in a series of tables and plots of the distributions of leveraged returns and arrival times. The complete results are given in tables, but are illustrated in a more easily understandable form for TTop Up in Section 7.1.

The most important results are summarised in Figures 7.5 to 7.12 which compare the proportion $p$ based Intensity Triangle sub-distributions with the Naive empirical results; and Cohen's d which shows the normalised effect size difference between Triangles and Naive results. The same Cohen's d values are shown in the right column of Tables 7.6 and 7.7, so as to compare with the underlying raw results in each table. While there are numerous other results reported these mostly report background validation details, whereas the two sets of results described above show the main measures which I use in the Conclusion.

Amongst related literature, Lo's paper is considered to use a large amount of data, where he studies time series sections of sample size 50, for a total of 250 stocks in 5 quintiles over years 1962 to 1996. I use far more data, studying time series sections of approximately 5000 stocks from 1960 to 2017. The number of stocks depends on how many are listed on the stock exchanges during that period, where many do not have enough liquidity to be used in the study. See Appendix 7 for more details of the data used. I deliberately make use of the maximum available data from CRSP as additional means to produce stronger evidence than the related literature to help produce more compelling evidence.

All non-discrete result measurements are accurate to three significant figures. Time $t$ or $\tau$ are measured in discrete trading days over time segment $B C$ and $h_{\sigma}$ sections are expressed in $\frac{h \text { price }}{\text { days }}$ units.

The $h_{\sigma} \in[6, \infty)$ results are included for completeness, but the $h_{\sigma} \in[0,3)$ and $h_{\sigma} \in[3,6)$ based results are considered more important. The $h_{\sigma} \in[6, \infty)$ results I consider less comparable due to the large variation of $h_{\sigma}$ and lower sample size, but it seems sensible 
to not discard this edge of the data and report fully.

\subsection{A Tail of TTop Up}

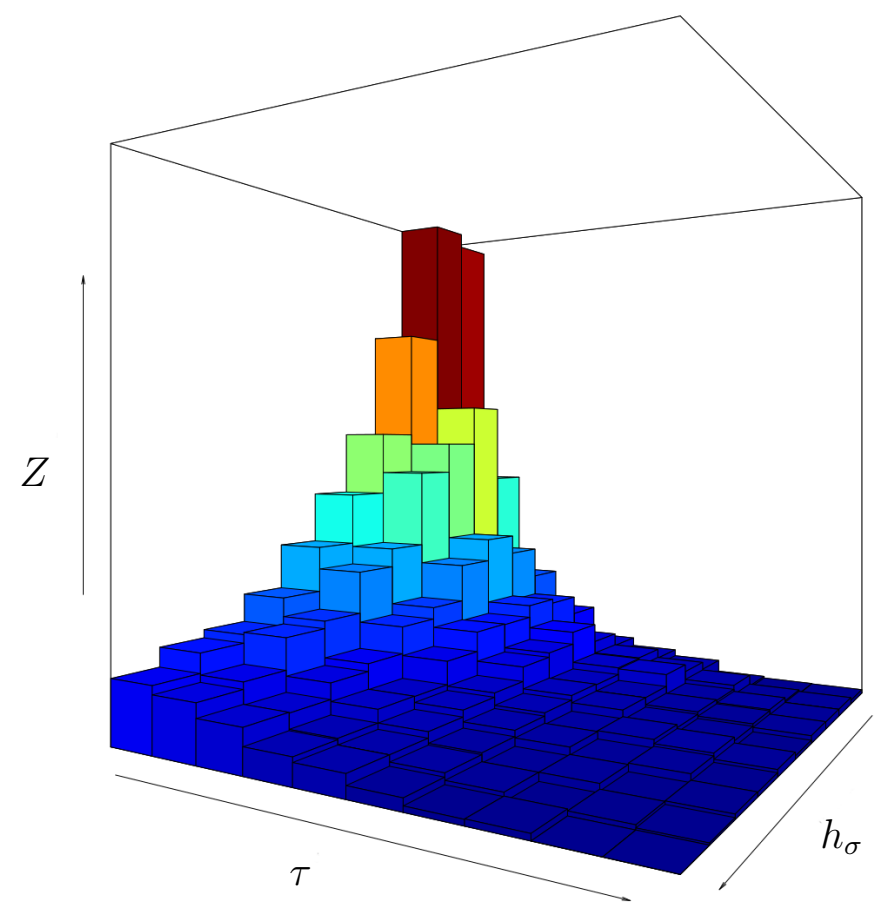

Figure 7.1: TTop Up 2D Distribution Over $h_{\sigma} \in(0,10], \tau \in(0,60]$

The US equity results require some thought to understand and this section provides more detail, mainly focusing on results for time period 1988 to 1998 and TTop Up where $h_{\sigma} \in$ $[0,3)$. Where possible I generalise to the wider set of results to provide greater insight.

An overview of the first passage time surface for 1988 to 1998 Triangles can be seen in Figure 7.1, which shows the arrival density decay across $h_{\sigma}$ slices as a histogram based surface of first passage densities in terms of $\left(h_{\sigma}, \tau\right)$, where the win and loss distributions are combined. From this surface it can be seen that there is a decay in arrival time density as $\tau$ increases and that the majority of the results are contained within the lower value $\left(h_{\sigma}, \tau\right)$ corner of the surface. All Triangle type distributions show similar distribution surfaces.

The two sets of results, from the Intensity model and Effect Size Heuristics, mainly differ in their inclusion of the right tail of $\tau$ or not. The Intensity model includes the right tail in order to model the whole arrival time $\tau$ distribution to gain contextual understanding (since the GEV is basically an extreme value distribution), while the Effect Heuristics cut off this tail at 10 days in order to solely evaluate the short term effects. The Effect Heuristics provide a far more transparent set of numerical results best summarised by Cohen's d but the Intensity model provides greater intuitive depth in the form of the 

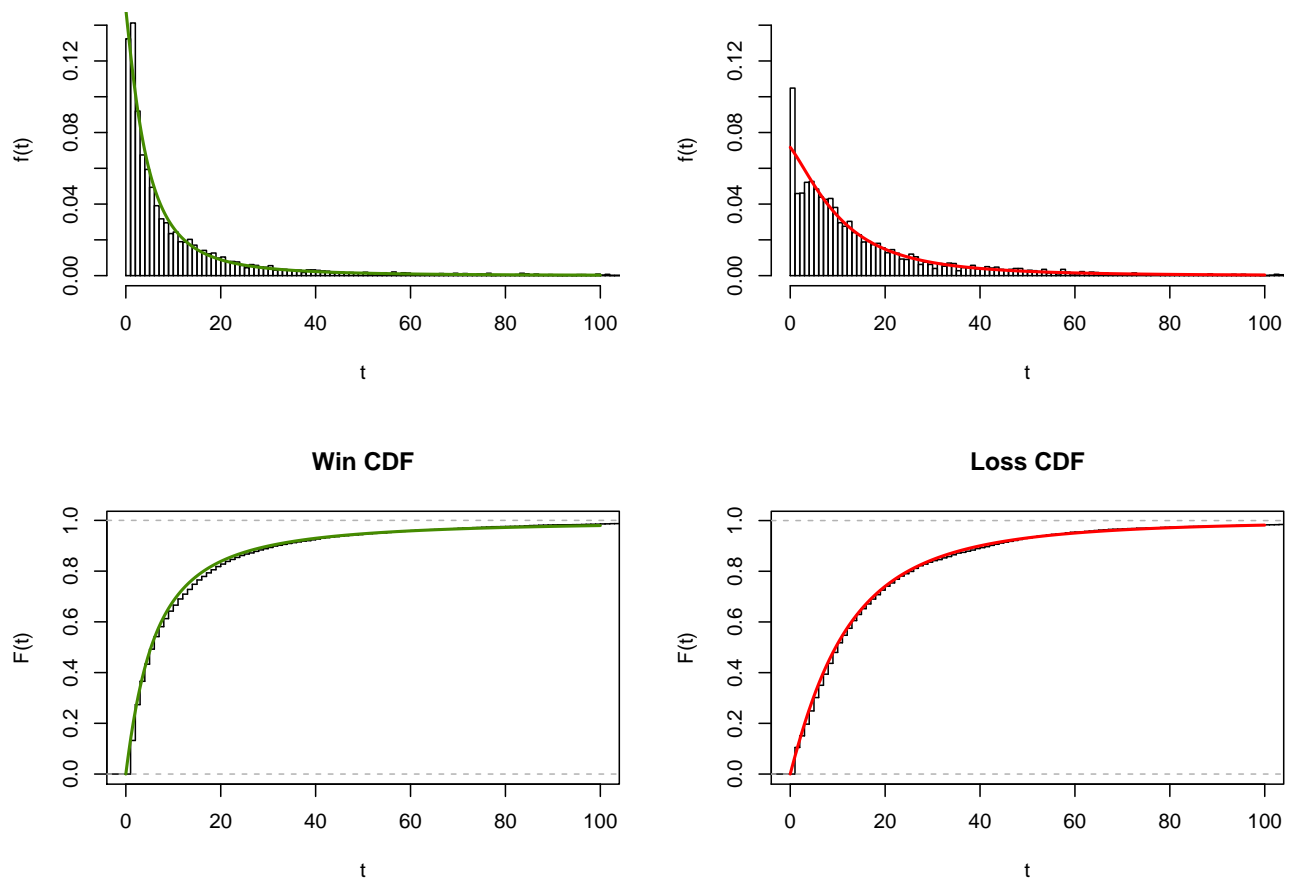

Figure 7.2: TTop Up In Sample 1988-1998 Triangle GEV Intensity vs Empirical Triangle Profile $h_{\sigma} \in[0,3)$

visual distribution comparison of Figures 7.5 to 7.12 .

The Intensity GEV model provides an explorative model of the win and loss first passage distribution, generally showing a very good fit for in-sample data, where the Triangle empirical stopping times cumulatively match the fitted model. For example Figure 7.2 shows this close distribution fit where the confirmation p-value is 0.998 for wins and 0.997 for losses; meaning the fit is exceptionally close. However this data is in-sample and proves little. Over the same period $p=0.631$, indicating larger mass in the win subdistribution. Consistently the model fits the in-sample data well for all Triangle cases as seen in Table 7.2, both for the sub-distributions and full distributions.

Because the Intensity GEV model cumulative fit is consistently nearly identical to the empirical Triangle data I compare the Triangle model fitted directly with Naive empirical in-sample data (in Figure 7.3) and Triangle forward out-of-sample data (in Figure 7.2). It is more concise to solely report the model based comparisons as the model fits the typical results closely.

Comparing the 1988-1998 Triangle model fit with the Naive data as seen in the profile plot of Figure 7.3 shows a curious result where the win distribution does not fit with a pvalue of 0.000 , and the loss distribution at least partly does fit with a p-value of 0.162 . To a strong degree, this difference is quite consistent across all of the patterns, except where 
Win PDF

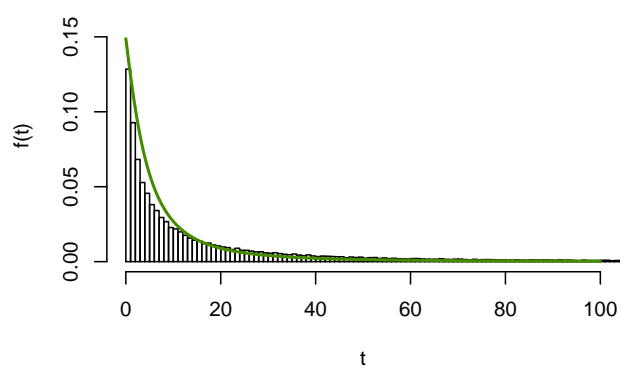

Win CDF

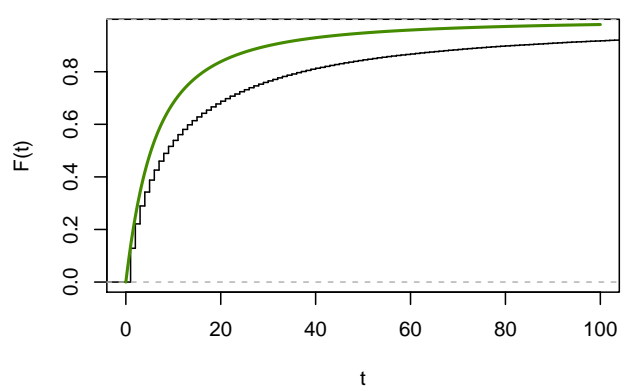

Loss PDF

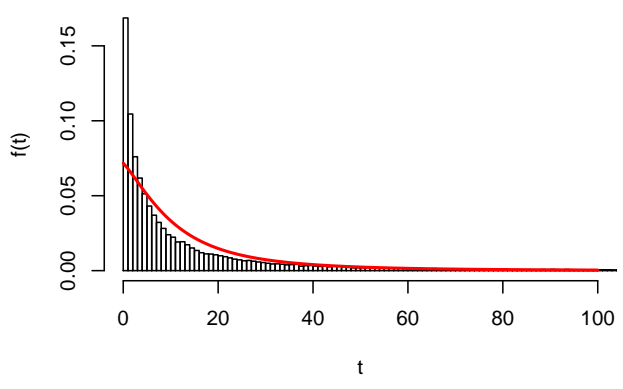

Loss CDF

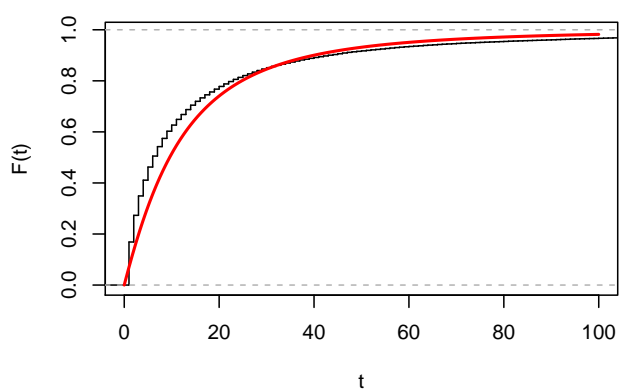

Figure 7.3: TTop Up In Sample 1988-1998 Triangle GEV Intensity vs Empirical Triangle Profile $h_{\sigma} \in[0,3)$

$h_{\sigma} \in[6, \infty)$. However the corresponding tests for the sub-distributions have $\mathrm{p}$-values close to 0 ; with most of these sub-distribution tests little insight can be gained due to proportion $p$ skewing the tests. What can be seen from the GEV Intensity fits is that there is a clear difference in the distribution of Win arrival times and less so for the loss arrival times. This is reasonably consistent across most cases except for $h_{\sigma} \in[0, \infty)$ as seen in Table 7.3. I suspect this probably means that winning trades are somehow fundamentally different between Triangle and Naive trades, and the losing trades are less so; however, it is hard to be more precise without further research.

The Intensity GEV Triangle modelling as compared with the Naive trades are best understood by reviewing Figures 7.5 to 7.12, in which these clearly show the gradually reducing effect of Triangles over time by showing the split cumulative probability of wins and losses. For 7.5 it can be seen that the period of 1988-1997 has a significantly higher green win CDF curve than the black line of Naive empirical trades indicating higher probability wins for Triangles; and the red loss curve is lower than the black line of the Naive empirical trades indicating lower probability losses for Triangles. This is useful as these plots give an indication of the variation in cumulative arrival time differences. The period of 1978-1987 shows a larger difference. The period of 1998-2008 shows a smaller difference. In general most Triangle types show that the effect has gradually decreased in each subsequent time section. 
Win PDF

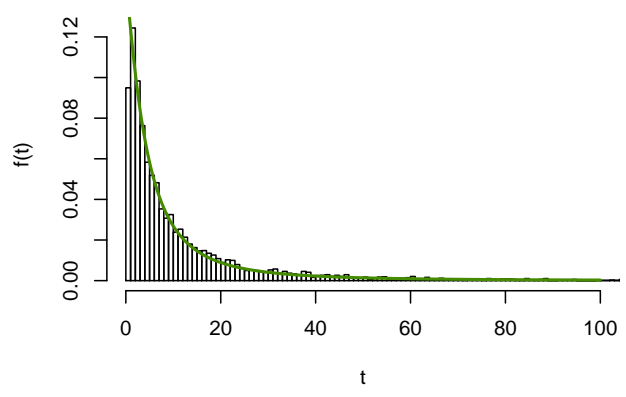

Win CDF

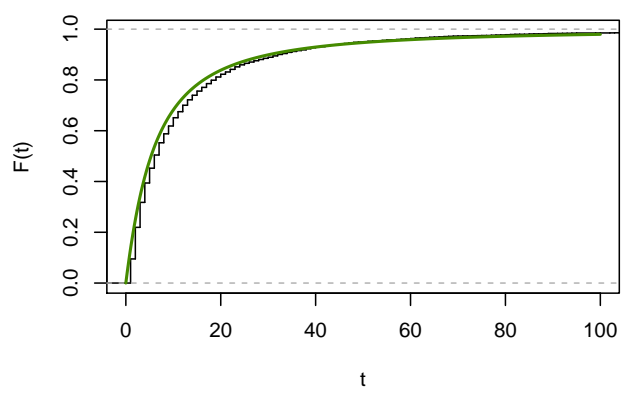

Loss PDF

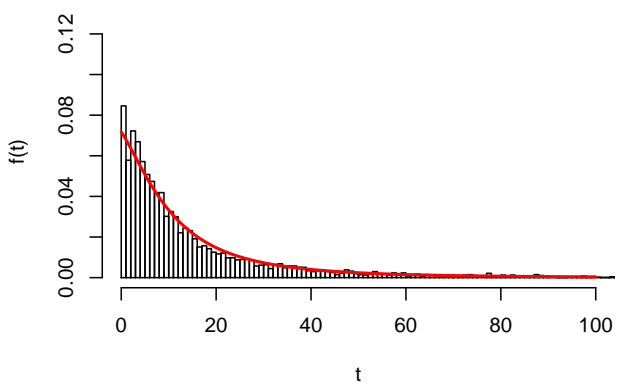

Loss CDF

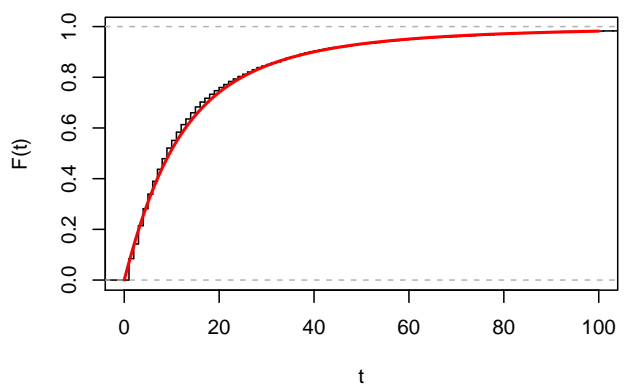

Figure 7.4: TTop Up 1988-1998 Triangle GEV Intensity vs 1998-2008 Forward Empirical Triangle Profile $h_{\sigma} \in[0,3)$

The difference in wins and losses can also be seen numerically as indicated by Bet return $r_{b}$ in Appendices B.1 and B.2. However the effect is much clearer when seen visually, also with the added insight of the time component. However the numerical measurement $r_{b}$ of pure winning and losing is of limited use due to the longer term systematic market effects likely hiding differences where the Triangles are of less effect.

Using the effect size heuristics which show the confidence intervals, the bet returns $r_{b}$ are $0.371[0.349,0.393]$ in 1988 to 1997 and $0.261[0.238,0.283]$ in 1998 to 2008 respectively. Taking into account arrival time and volatility for risk Cohen's d decreases from $0.304[0.282,0.327]$ to $0.221[0.202,0.241]$ over corresponding time periods. Overall Cohen's d decreases similarly across most results in subsequent time periods, showing that the competitive advantages of Triangles have decreased with time and that when normalised by volatility, most Triangle types are now probably of negligible information value. This confirms the reducing effect seen in Figures 7.5 to 7.12.

When the model is fitted over 1988 to 1998 Triangles, then tested against forward 1998 2008 empirical Triangle data the fit p-values are 0.974 for wins and 0.973 for losses respectively. These are lower than in-sample fits but still surprisingly high. Visually this good fit is confirmed in Figure 7.4. It seems the fitted distribution of first passage times predicts the future distribution quite well, and this is generally the case across most results. 
There is an issue with the proportional distribution tests of sub-distributions denoted by $p G_{T 1}(t)$ and $(1-p) G_{T 2}(t)$ in the results tables. Neither of these are true distributions and so cumulatively over $(0, \infty]$ these sum to a value of $p$ or $1-p$, which are both less than one. Consequently when tested cumulatively against empirical distributions, where the proportionate value $p$ differs between the model and empirical results, the test will often quickly converge toward 0 . While these cumulative deviations test results are correct for these sub-distributions, the common close to zero result conveys little information about how the sub-distributions differ. It would be better to devise a measure which communicates how these results differ over time and for the most part the sub-distribution plots display this difference well in Figures 7.5 to 7.12..

\subsection{Intensity Model}

For reference, the GEV Intensity distribution likelihood function is characterized by location $\mu_{1}, \mu_{2}$ scale $\sigma_{1}, \sigma_{2}$ and shape $\xi_{1}, \xi_{2}$ as

$$
\begin{aligned}
\mathcal{L}(t, t+d t) & =\left[f^{(+)}(t)\right]^{\delta}\left[f^{(-)}(t)\right]^{1-\delta} \\
& =\left[p g_{T 1}(t)\right]^{\delta}\left[(1-p) g_{T 2}(t)\right]^{1-\delta} \\
& =\left[\frac{\frac{p}{\sigma_{1}}\left(1+\xi_{1} \frac{t-\mu_{1}}{\sigma_{1}}\right)^{\frac{-1}{\xi_{1}}-1} e^{-\left(1+\xi_{1} \frac{t-\mu_{1}}{\sigma_{1}}\right)^{\frac{-1}{\xi_{1}}}}}{1-e^{-\left(1+\xi_{1} \frac{t-\mu_{1}}{\sigma_{1}}\right)^{\frac{-1}{\xi_{1}}}}}\right]^{\delta}\left[\frac{\frac{(1-p)}{\sigma_{2}}\left(1+\xi_{2} \frac{t-\mu_{2}}{\sigma_{2}}\right)^{\frac{-1}{\xi_{2}}-1} e^{-\left(1+\xi_{2} \frac{t-\mu_{2}}{\sigma_{2}}\right)^{\frac{-1}{\xi_{2}}}}}{1-e^{-\left(1+\xi_{2} \frac{t-\mu_{2}}{\sigma_{2}}\right)^{\frac{-1}{\xi_{2}}}}}\right]^{1-\delta},
\end{aligned}
$$

has parameter values which correspond to the estimates found in Table 7.1.

Distributions are denoted by $p G_{T 1}(t), G_{T 1}(t),(1-p) G_{T 2}(t)$ and $G_{T 2}(t)$, as indicated by (5.13), (5.14) and (7.1) which show the likelihood equation relationship; corresponding tests based on cumulative deviation are found in Tables 7.2 to 7.4.

For the forward testing, Table 7.4 shows the p-values in yearly time periods which represent the period that the model was fitted over (the test being against the next time period section). To make this clear where there is no fitted model tested in the last time period of 2008 to 2018 NA is reported, since the model was fitted in 1998 to 2008 and tested against the 2008 to 2018 Triangle data, showing the p-values in the 1998 to 2008 table cell.

These results generally show Triangles with a positive probability of winning but this decreases over the measured periods from 1960 to 2018 as compared with the Naive case. Testing the model fit against Triangle in-sample data shows exceptionally good fit. Testing against Naive data generally shows a poor fit for wins, and a good fit for losses; indicating that the winning Triangle trades have a different arrival time distribution.

Curiously despite not discounting systematic market effects, forward goodness of fit 
testing shows that the first passage times have a predictive capacity in first passage times, though this is less clear with the sub-distribution tests due to skewed testing issues.

Table 7.1: US Equity GEV Model Parameter Estimates

\begin{tabular}{|c|c|c|c|c|c|c|c|c|c|}
\hline \multirow[b]{3}{*}{ Type } & \multirow[b]{3}{*}{$h_{\sigma}$} & \multirow[b]{3}{*}{ Period } & \multicolumn{7}{|c|}{ Intensity GEV Model Parameters } \\
\hline & & & \multicolumn{3}{|c|}{ Win Distribution } & \multicolumn{3}{|c|}{ Loss Distribution } & \multirow[b]{2}{*}{$p$} \\
\hline & & & $\mu_{1}$ & $\sigma_{1}$ & $\xi_{1}$ & $\mu_{2}$ & $\sigma_{2}$ & $\xi_{2}$ & \\
\hline TTop Up & {$\left[\begin{array}{lll}0 & 3\end{array}\right)$} & 1960-01-01 1978-01-01 & 3.09 & 3.67 & 0.886 & 6.9 & 9.35 & 0.434 & 0.647 \\
\hline TTop Up & {$[03)$} & 1978-01-01 1988-01-01 & 3.47 & 4.36 & 0.803 & 5.59 & 9.31 & 0.383 & 0.664 \\
\hline TTop Up & {$\left[\begin{array}{lll}0 & 3\end{array}\right)$} & 1988-01-01 1998-01-01 & 0 & 3.91 & 0.666 & 0 & 8.11 & 0.396 & 0.631 \\
\hline TTop Up & {$[03)$} & 1998-01-01 2008-01-01 & 2.98 & 4.73 & 0.678 & 1 & 7.16 & 0.465 & 0.592 \\
\hline TTop Up & {$\left[\begin{array}{lll}0 & 3\end{array}\right)$} & 2008-01-01 2018-01-01 & 2.89 & 6.3 & 0.521 & 4.48 & 6.53 & 0.517 & 0.571 \\
\hline TTop Up & {$[36)$} & 1960-01-01 1978-01-01 & 5.4 & 5.94 & 0.801 & 11 & 10.5 & 0.45 & 0.611 \\
\hline TTop Up & {$[36)$} & 1978-01-01 1988-01-01 & 2.32 & 8.06 & 0.479 & 10.3 & 10.2 & 0.484 & 0.627 \\
\hline TTop Up & {$[36)$} & 1988-01-01 1998-01-01 & 5.83 & 7.55 & 0.596 & 9.21 & 10.5 & 0.454 & 0.611 \\
\hline TTop Up & {$[36)$} & 1998-01-01 2008-01-01 & 6.03 & 8.51 & 0.542 & 7.89 & 8.81 & 0.522 & 0.567 \\
\hline TTop Up & {$[36)$} & 2008-01-01 2018-01-01 & 7.75 & 9.3 & 0.506 & 7.4 & 7.77 & 0.566 & 0.539 \\
\hline TTop Up & {$[6 \infty)$} & 1960-01-01 1978-01-01 & 7.66 & 9.33 & 0.636 & 17.2 & 14.9 & 0.48 & 0.559 \\
\hline TTop Up & {$[6 \infty)$} & 1978-01-01 1988-01-01 & 1 & 12.3 & 0.416 & 15.1 & 14.2 & 0.52 & 0.605 \\
\hline TTop Up & {$[6 \infty)$} & 1988-01-01 1998-01-01 & 1.7 & 12.9 & 0.413 & 12.5 & 13.7 & 0.417 & 0.587 \\
\hline TTop Up & {$[6 \infty)$} & 1998-01-01 2008-01-01 & 9.67 & 12.4 & 0.5 & 10.8 & 11.4 & 0.57 & 0.529 \\
\hline TTop Up & {$[6 \infty)$} & 2008-01-01 2018-01-01 & 10.5 & 11.9 & 0.471 & 9.22 & 10 & 0.535 & 0.547 \\
\hline TTop Down & {$\left[\begin{array}{lll}0 & 3\end{array}\right)$} & 1960-01-01 1978-01-01 & 5.71 & 7.66 & 0.561 & 7.98 & 9.23 & 0.578 & 0.558 \\
\hline TTop Down & [0 3) & 1978-01-01 1988-01-01 & 5.24 & 6.77 & 0.654 & 7.24 & 9.57 & 0.499 & 0.547 \\
\hline TTop Down & {$\left[\begin{array}{lll}0 & 3\end{array}\right)$} & 1988-01-01 1998-01-01 & 0.5 & 7.01 & 0.543 & 2.58 & 8.96 & 0.508 & 0.539 \\
\hline TTop Down & [0 3) & 1998-01-01 2008-01-01 & 4.5 & 7.71 & 0.597 & 0.974 & 7.67 & 0.579 & 0.493 \\
\hline TTop Down & {$\left[\begin{array}{ll}0 & 3\end{array}\right)$} & 2008-01-01 2018-01-01 & 5.66 & 6.55 & 0.796 & 3.67 & 7.42 & 0.614 & 0.478 \\
\hline TTop Down & {$[36)$} & 1960-01-01 1978-01-01 & 8.12 & 8.81 & 0.645 & 10.8 & 10.6 & 0.569 & 0.556 \\
\hline TTop Down & {$[36)$} & 1978-01-01 1988-01-01 & 7.81 & 9.21 & 0.606 & 11.6 & 11.7 & 0.562 & 0.54 \\
\hline TTop Down & {$[36)$} & 1988-01-01 1998-01-01 & 6.05 & 9.01 & 0.578 & 10.1 & 10.6 & 0.583 & 0.521 \\
\hline TTop Down & {$[36)$} & 1998-01-01 2008-01-01 & 7.1 & 8.66 & 0.598 & 8.74 & 9.9 & 0.611 & 0.495 \\
\hline TTop Down & {$[36)$} & 2008-01-01 2018-01-01 & 6.89 & 7.53 & 0.703 & 7.56 & 8.88 & 0.659 & 0.488 \\
\hline TTop Down & {$[6 \infty)$} & 1960-01-01 1978-01-01 & 3.52 & 15.6 & 0.359 & 14.2 & 12.9 & 0.547 & 0.585 \\
\hline TTop Down & {$[6 \infty)$} & 1978-01-01 1988-01-01 & 10.4 & 11.3 & 0.582 & 14.5 & 15 & 0.471 & 0.567 \\
\hline TTop Down & {$[6 \infty)$} & 1988-01-01 1998-01-01 & 9.9 & 10.8 & 0.756 & 12.7 & 14.7 & 0.583 & 0.522 \\
\hline TTop Down & {$[6 \infty)$} & 1998-01-01 2008-01-01 & 9.7 & 11.4 & 0.659 & 11.8 & 12.8 & 0.586 & 0.52 \\
\hline TTop Down & {$[6 \infty)$} & 2008-01-01 2018-01-01 & 8.21 & 8.89 & 0.718 & 9.54 & 10.9 & 0.583 & 0.479 \\
\hline TBot Up & {$\left[\begin{array}{lll}0 & 3\end{array}\right)$} & 1960-01-01 1978-01-01 & 0 & 4.31 & 0.603 & 5.55 & 9.71 & 0.407 & 0.618 \\
\hline TBot Up & {$\left[\begin{array}{lll}0 & 3\end{array}\right)$} & 1978-01-01 1988-01-01 & 0 & 4.65 & 0.6 & 3.62 & 10.2 & 0.387 & 0.658 \\
\hline TBot Up & {$\left[\begin{array}{lll}0 & 3\end{array}\right)$} & 1988-01-01 1998-01-01 & 0 & 3.95 & 0.696 & 0 & 8.93 & 0.427 & 0.619 \\
\hline TBot Up & {$\left[\begin{array}{lll}0 & 3\end{array}\right)$} & 1998-01-01 2008-01-01 & 0 & 5.21 & 0.579 & 0 & 8.3 & 0.44 & 0.575 \\
\hline TBot Up & {$[03)$} & 2008-01-01 2018-01-01 & 0.5 & 6.48 & 0.526 & 3.1 & 8.12 & 0.432 & 0.546 \\
\hline TBot Up & {$[36)$} & 1960-01-01 1978-01-01 & 5.61 & 6.99 & 0.652 & 11.3 & 11.4 & 0.383 & 0.574 \\
\hline TBot Up & {$[36)$} & 1978-01-01 1988-01-01 & 1 & 9.08 & 0.421 & 12 & 11.8 & 0.417 & 0.618 \\
\hline TBot Up & {$[36)$} & 1988-01-01 1998-01-01 & 5.96 & 8.36 & 0.551 & 10.2 & 11 & 0.479 & 0.606 \\
\hline
\end{tabular}


Table 7.1: US Equity GEV Model Parameter Estimates (continued)

\begin{tabular}{llllllllll}
\hline Type & $h_{\sigma}$ & Period & $\mu_{1}$ & $\sigma_{1}$ & $\xi_{1}$ & $\mu_{2}$ & $\sigma_{2}$ & $\xi_{2}$ & $p$ \\
\hline TBot Up & {$[36)$} & $1998-01-012008-01-01$ & 5.93 & 9.36 & 0.516 & 7.73 & 10.9 & 0.428 & 0.56 \\
TBot Up & {$[36)$} & $2008-01-012018-01-01$ & 8.2 & 9.88 & 0.572 & 7.84 & 8.81 & 0.536 & 0.525 \\
& & & & & & & & & \\
TBot Up & {$[6 \infty)$} & $1960-01-011978-01-01$ & 7.56 & 8.62 & 0.726 & 14.4 & 12.6 & 0.458 & 0.561 \\
TBot Up & {$[6 \infty)$} & $1978-01-011988-01-01$ & 0.683 & 12 & 0.359 & 14.6 & 13 & 0.388 & 0.591 \\
TBot Up & {$[6 \infty)$} & $1988-01-011998-01-01$ & 6.74 & 10.6 & 0.521 & 12.2 & 13.6 & 0.438 & 0.584 \\
TBot Up & {$[6 \infty)$} & $1998-01-012008-01-01$ & 8.02 & 11.6 & 0.501 & 12.4 & 13.6 & 0.495 & 0.517 \\
TBot Up & {$[6 \infty)$} & $2008-01-012018-01-01$ & 10.7 & 11.9 & 0.639 & 9.59 & 9.68 & 0.655 & 0.526 \\
TBot Down & {$[03)$} & $1960-01-011978-01-01$ & 0 & 8.65 & 0.408 & 7.43 & 9 & 0.547 & 0.57 \\
TBot Down & {$[03)$} & $1978-01-011988-01-01$ & 0 & 7.44 & 0.53 & 6.9 & 9.59 & 0.538 & 0.538 \\
TBot Down & {$[03)$} & $1988-01-011998-01-01$ & 0 & 7.02 & 0.589 & 0 & 8.35 & 0.537 & 0.538 \\
TBot Down & {$[03)$} & $1998-01-012008-01-01$ & 1 & 8.37 & 0.55 & 2.28 & 8.41 & 0.598 & 0.523 \\
TBot Down & {$[03)$} & $2008-01-012018-01-01$ & 5.63 & 6.97 & 0.695 & 4.5 & 8.44 & 0.566 & 0.485 \\
TBot Down & {$[36)$} & $1960-01-011978-01-01$ & 8.39 & 9.66 & 0.531 & 10.9 & 10.8 & 0.532 & 0.577 \\
TBot Down & {$[36)$} & $1978-01-011988-01-01$ & 1 & 11.9 & 0.377 & 11.9 & 11.8 & 0.564 & 0.547 \\
TBot Down & {$[36)$} & $1988-01-011998-01-01$ & 6.26 & 9.55 & 0.516 & 10.4 & 10.7 & 0.56 & 0.528 \\
TBot Down & {$[36)$} & $1998-01-012008-01-01$ & 7.46 & 8.96 & 0.68 & 8.8 & 9.95 & 0.627 & 0.519 \\
TBot Down & {$[36)$} & $2008-01-012018-01-01$ & 6.75 & 7 & 0.725 & 7.97 & 10.4 & 0.524 & 0.489 \\
TBot Down & {$[6 \infty)$} & $1960-01-011978-01-01$ & 10.4 & 10.6 & 0.586 & 13.6 & 12.5 & 0.531 & 0.59 \\
TBot Down & {$[6 \infty)$} & $1978-01-011988-01-01$ & 6.79 & 13.5 & 0.429 & 13.7 & 13.1 & 0.561 & 0.572 \\
TBot Down & {$[6 \infty)$} & $1988-01-011998-01-01$ & 1.68 & 15 & 0.427 & 11.9 & 12.8 & 0.574 & 0.524 \\
TBot Down & {$[6 \infty)$} & $1998-01-012008-01-01$ & 10.4 & 12.9 & 0.613 & 12 & 12.6 & 0.628 & 0.531 \\
TBot Down & {$[6 \infty)$} & $2008-01-012018-01-01$ & 7.77 & 8.04 & 0.703 & 10.2 & 10.5 & 0.605 & 0.489 \\
\hline
\end{tabular}

Table 7.2: US Equity In-Sample Triangle Cumulative Deviations Tests

\begin{tabular}{|c|c|c|c|c|c|c|}
\hline \multirow[b]{3}{*}{ Type } & \multirow[b]{3}{*}{$h_{\sigma}$} & \multirow[b]{3}{*}{ Period } & \multicolumn{4}{|c|}{ p-value } \\
\hline & & & \multicolumn{2}{|c|}{ Win Distribution } & \multicolumn{2}{|c|}{ Loss Distribution } \\
\hline & & & $p G_{T 1}(t)$ & $G_{T 1}(t)$ & $(1-p) G_{T 2}(t)$ & $G_{T 2}(t)$ \\
\hline TTop Up & {$\left[\begin{array}{lll}0 & 3\end{array}\right)$} & 1960-01-01 1978-01-01 & 0.926 & 0.930 & 0.969 & 0.965 \\
\hline TTop Up & {$\left[\begin{array}{lll}0 & 3\end{array}\right)$} & 1978-01-01 1988-01-01 & 0.871 & 0.872 & 0.971 & 0.972 \\
\hline TTop Up & {$\left[\begin{array}{lll}0 & 3\end{array}\right)$} & 1988-01-01 1998-01-01 & 0.998 & 0.997 & 0.992 & 0.994 \\
\hline TTop Up & {$\left[\begin{array}{lll}0 & 3\end{array}\right)$} & 1998-01-01 2008-01-01 & 0.953 & 0.954 & 0.996 & 0.996 \\
\hline TTop Up & [0 3) & 2008-01-01 2018-01-01 & 0.993 & 0.992 & 0.968 & 0.968 \\
\hline TTop Up & {$[36)$} & 1960-01-01 1978-01-01 & 0.889 & 0.889 & 0.897 & 0.899 \\
\hline TTop Up & {$[36)$} & 1978-01-01 1988-01-01 & 0.914 & 0.915 & 0.996 & 0.995 \\
\hline TTop Up & {$[36)$} & 1988-01-01 1998-01-01 & 0.897 & 0.897 & 0.934 & 0.936 \\
\hline TTop Up & {$[36)$} & 1998-01-01 2008-01-01 & 0.978 & 0.976 & 0.948 & 0.952 \\
\hline TTop Up & {$[36)$} & 2008-01-01 2018-01-01 & 0.962 & 0.963 & 0.988 & 0.988 \\
\hline TTop Up & {$[6 \infty)$} & 1960-01-01 1978-01-01 & 0.970 & 0.855 & 0.740 & 0.868 \\
\hline TTop Up & {$[6 \infty)$} & 1978-01-01 1988-01-01 & 0.985 & 0.980 & 0.967 & 0.973 \\
\hline TTop Up & {$[6 \infty)$} & 1988-01-01 1998-01-01 & 0.977 & 0.977 & 0.921 & 0.921 \\
\hline TTop Up & {$[6 \infty)$} & 1998-01-01 2008-01-01 & 0.905 & 0.873 & 0.976 & 0.978 \\
\hline
\end{tabular}


Table 7.2: US Equity In-Sample Triangle Cumulative Deviations Tests (continued)

\begin{tabular}{|c|c|c|c|c|c|c|}
\hline Type & $h_{\sigma}$ & Period & $p G_{T 1}(t)$ & $G_{T 1}(t)$ & $(1-p) G_{T 2}(t)$ & $G_{T 2}(t)$ \\
\hline TTop Up & {$[6 \infty)$} & 2008-01-01 2018-01-01 & 0.928 & 0.860 & 0.912 & 0.920 \\
\hline TTop Down & [0 3) & 1960-01-01 1978-01-01 & 0.854 & 0.853 & 0.981 & 0.983 \\
\hline TTop Down & {$[03)$} & 1978-01-01 1988-01-01 & 0.975 & 0.978 & 0.995 & 0.992 \\
\hline TTop Down & {$\left[\begin{array}{lll}0 & 3\end{array}\right)$} & 1988-01-01 1998-01-01 & 0.971 & 0.976 & 0.984 & 0.984 \\
\hline TTop Down & {$[03)$} & 1998-01-01 2008-01-01 & 0.974 & 0.974 & 0.986 & 0.888 \\
\hline TTop Down & [0 3) & 2008-01-01 2018-01-01 & 0.926 & 0.927 & 0.973 & 0.973 \\
\hline TTop Down & {$[36)$} & 1960-01-01 1978-01-01 & 0.905 & 0.906 & 0.903 & 0.903 \\
\hline TTop Down & {$[36)$} & 1978-01-01 1988-01-01 & 0.859 & 0.859 & 0.959 & 0.960 \\
\hline TTop Down & {$[36)$} & 1988-01-01 1998-01-01 & 0.898 & 0.898 & 0.945 & 0.946 \\
\hline TTop Down & {$[36)$} & 1998-01-01 2008-01-01 & 0.957 & 0.958 & 0.872 & 0.871 \\
\hline TTop Down & {$[36)$} & 2008-01-01 2018-01-01 & 0.843 & 0.844 & 0.953 & 0.954 \\
\hline TTop Down & {$[6 \infty)$} & 1960-01-01 1978-01-01 & 0.945 & 0.947 & 0.999 & 1.000 \\
\hline TTop Down & {$[6 \infty)$} & 1978-01-01 1988-01-01 & 0.914 & 0.915 & 0.996 & 0.996 \\
\hline TTop Down & {$[6 \infty)$} & 1988-01-01 1998-01-01 & 0.838 & 0.913 & 0.950 & 0.971 \\
\hline TTop Down & {$[6 \infty)$} & 1998-01-01 2008-01-01 & 0.982 & 0.948 & 0.990 & 0.753 \\
\hline TTop Down & {$[6 \infty)$} & 2008-01-01 2018-01-01 & 0.933 & 0.933 & 0.968 & 0.993 \\
\hline TBot Up & {$[03)$} & 1960-01-01 1978-01-01 & 0.953 & 0.959 & 0.996 & 0.999 \\
\hline TBot Up & {$[03)$} & 1978-01-01 1988-01-01 & 0.956 & 0.958 & 0.990 & 0.988 \\
\hline TBot Up & {$[03)$} & 1988-01-01 1998-01-01 & 0.905 & 0.907 & 0.981 & 0.981 \\
\hline TBot Up & {$[03)$} & 1998-01-01 2008-01-01 & 0.996 & 0.995 & 0.965 & 0.965 \\
\hline TBot Up & {$\left[\begin{array}{lll}0 & 3\end{array}\right)$} & 2008-01-01 2018-01-01 & 0.951 & 0.952 & 0.998 & 0.998 \\
\hline TBot Up & {$[36)$} & 1960-01-01 1978-01-01 & 0.973 & 0.973 & 0.894 & 0.896 \\
\hline TBot Up & {$[36)$} & 1978-01-01 1988-01-01 & 0.965 & 0.965 & 0.983 & 0.984 \\
\hline TBot Up & {$[36)$} & 1988-01-01 1998-01-01 & 0.936 & 0.918 & 0.915 & 0.939 \\
\hline TBot Up & {$[36)$} & 1998-01-01 2008-01-01 & 0.819 & 0.834 & 0.998 & 0.987 \\
\hline TBot Up & {$[36)$} & 2008-01-01 2018-01-01 & 0.947 & 0.953 & 0.940 & 0.935 \\
\hline TBot Up & {$[6 \infty)$} & 1960-01-01 1978-01-01 & 0.880 & 0.880 & 0.990 & 0.991 \\
\hline TBot Up & {$[6 \infty)$} & 1978-01-01 1988-01-01 & 0.975 & 0.974 & 0.956 & 0.957 \\
\hline TBot Up & {$[6 \infty)$} & 1988-01-01 1998-01-01 & 0.917 & 0.885 & 0.869 & 0.907 \\
\hline TBot Up & {$[6 \infty)$} & 1998-01-01 2008-01-01 & 0.879 & 0.881 & 0.904 & 0.903 \\
\hline TBot Up & {$[6 \infty)$} & 2008-01-01 2018-01-01 & 0.900 & 0.977 & 0.940 & 0.942 \\
\hline TBot Down & {$[03)$} & 1960-01-01 1978-01-01 & 0.995 & 0.996 & 0.999 & 0.998 \\
\hline TBot Down & {$[03)$} & 1978-01-01 1988-01-01 & 0.952 & 0.949 & 0.982 & 0.985 \\
\hline TBot Down & {$[03)$} & 1988-01-01 1998-01-01 & 0.904 & 0.678 & 0.998 & 0.899 \\
\hline TBot Down & {$\left[\begin{array}{ll}0 & 3\end{array}\right)$} & 1998-01-01 2008-01-01 & 0.951 & 0.704 & 0.985 & 0.958 \\
\hline TBot Down & {$\left[\begin{array}{ll}0 & 3\end{array}\right)$} & 2008-01-01 2018-01-01 & 0.818 & 0.821 & 0.937 & 0.866 \\
\hline TBot Down & {$[36)$} & 1960-01-01 1978-01-01 & 0.841 & 0.834 & 0.929 & 0.939 \\
\hline TBot Down & {$[36)$} & 1978-01-01 1988-01-01 & 0.968 & 0.967 & 0.738 & 0.741 \\
\hline TBot Down & {$[36)$} & 1988-01-01 1998-01-01 & 0.942 & 0.943 & 0.911 & 0.911 \\
\hline TBot Down & {$[36)$} & 1998-01-01 2008-01-01 & 0.952 & 0.952 & 0.963 & 0.963 \\
\hline TBot Down & [3 6) & 2008-01-01 2018-01-01 & 0.971 & 0.970 & 0.971 & 0.972 \\
\hline TBot Down & {$[6 \infty)$} & 1960-01-01 1978-01-01 & 0.980 & 0.980 & 0.893 & 0.893 \\
\hline TBot Down & {$[6 \infty)$} & 1978-01-01 1988-01-01 & 0.981 & 0.980 & 0.983 & 0.984 \\
\hline TBot Down & {$[6 \infty)$} & 1988-01-01 1998-01-01 & 0.986 & 0.986 & 0.943 & 0.944 \\
\hline
\end{tabular}


Table 7.2: US Equity In-Sample Triangle Cumulative Deviations Tests (continued)

\begin{tabular}{lllrrrr}
\hline Type & $h_{\sigma}$ & Period & $p G_{T 1}(t)$ & $G_{T 1}(t)$ & $(1-p) G_{T 2}(t)$ & $G_{T 2}(t)$ \\
\hline TBot Down & {$[6 \infty)$} & 1998-01-01 2008-01-01 & 0.990 & 0.634 & 0.970 & 0.920 \\
TBot Down & {$[6 \infty)$} & 2008-01-01 2018-01-01 & 0.955 & 0.959 & 0.956 & 0.952 \\
\hline
\end{tabular}

Table 7.3: US Equity In Sample Naive Event Cumulative Deviations Tests

\begin{tabular}{|c|c|c|c|c|c|c|}
\hline \multirow[b]{3}{*}{ Type } & \multirow[b]{3}{*}{$h_{\sigma}$} & \multirow[b]{3}{*}{ Period } & \multicolumn{4}{|c|}{ p-value } \\
\hline & & & \multicolumn{2}{|c|}{ Win Distribution } & \multicolumn{2}{|c|}{ Loss Distribution } \\
\hline & & & $p G_{T 1}(t)$ & $G_{T 1}(t)$ & $(1-p) G_{T 2}(t)$ & $G_{T 2}(t)$ \\
\hline TTop Up & [0 3) & 1960-01-01 1978-01-01 & 0.000 & 0.000 & 0.000 & 0.353 \\
\hline TTop Up & {$\left[\begin{array}{lll}0 & 3\end{array}\right)$} & 1978-01-01 1988-01-01 & 0.000 & 0.000 & 0.000 & 0.255 \\
\hline TTop Up & [0 3) & 1988-01-01 1998-01-01 & 0.000 & 0.000 & 0.000 & 0.162 \\
\hline TTop Up & {$\left[\begin{array}{lll}0 & 3\end{array}\right)$} & 1998-01-01 2008-01-01 & 0.000 & 0.000 & 0.000 & 0.445 \\
\hline TTop Up & {$\left[\begin{array}{lll}0 & 3\end{array}\right)$} & 2008-01-01 2018-01-01 & 0.000 & 0.024 & 0.000 & 0.654 \\
\hline TTop Up & {$[36)$} & 1960-01-01 1978-01-01 & 0.000 & 0.000 & 0.000 & 0.159 \\
\hline TTop Up & {$[36)$} & 1978-01-01 1988-01-01 & 0.000 & 0.000 & 0.000 & 0.187 \\
\hline TTop Up & {$[36)$} & 1988-01-01 1998-01-01 & 0.009 & 0.000 & 0.001 & 0.122 \\
\hline TTop Up & {$[36)$} & 1998-01-01 2008-01-01 & 0.000 & 0.000 & 0.000 & 0.217 \\
\hline TTop Up & {$[36)$} & 2008-01-01 2018-01-01 & 0.002 & 0.007 & 0.001 & 0.643 \\
\hline TTop Up & {$[6 \infty)$} & 1960-01-01 1978-01-01 & 0.000 & 0.000 & 0.000 & 0.010 \\
\hline TTop Up & {$[6 \infty)$} & 1978-01-01 1988-01-01 & 0.000 & 0.000 & 0.000 & 0.001 \\
\hline TTop Up & {$[6 \infty)$} & 1988-01-01 1998-01-01 & 0.000 & 0.000 & 0.000 & 0.003 \\
\hline TTop Up & {$[6 \infty)$} & 1998-01-01 2008-01-01 & 0.000 & 0.000 & 0.000 & 0.000 \\
\hline TTop Up & {$[6 \infty)$} & 2008-01-01 2018-01-01 & 0.000 & 0.000 & 0.000 & 0.000 \\
\hline TTop Down & [0 3) & 1960-01-01 1978-01-01 & 0.000 & 0.000 & 0.000 & 0.638 \\
\hline TTop Down & {$\left[\begin{array}{lll}0 & 3\end{array}\right)$} & 1978-01-01 1988-01-01 & 0.000 & 0.000 & 0.000 & 0.492 \\
\hline TTop Down & {$\left[\begin{array}{lll}0 & 3\end{array}\right)$} & 1988-01-01 1998-01-01 & 0.000 & 0.000 & 0.000 & 0.262 \\
\hline TTop Down & {$\left[\begin{array}{lll}0 & 3\end{array}\right)$} & 1998-01-01 2008-01-01 & 0.000 & 0.000 & 0.000 & 0.854 \\
\hline TTop Down & {$\left[\begin{array}{lll}0 & 3\end{array}\right)$} & 2008-01-01 2018-01-01 & 0.004 & 0.067 & 0.009 & 0.121 \\
\hline TTop Down & {$[36)$} & 1960-01-01 1978-01-01 & 0.000 & 0.000 & 0.000 & 0.105 \\
\hline TTop Down & {$[36)$} & 1978-01-01 1988-01-01 & 0.000 & 0.000 & 0.000 & 0.182 \\
\hline TTop Down & {$[36)$} & 1988-01-01 1998-01-01 & 0.000 & 0.000 & 0.000 & 0.159 \\
\hline TTop Down & {$[36)$} & 1998-01-01 2008-01-01 & 0.000 & 0.000 & 0.000 & 0.289 \\
\hline TTop Down & {$[36)$} & 2008-01-01 2018-01-01 & 0.000 & 0.042 & 0.000 & 0.974 \\
\hline TTop Down & {$[6 \infty)$} & 1960-01-01 1978-01-01 & 0.000 & 0.000 & 0.000 & 0.000 \\
\hline TTop Down & {$[6 \infty)$} & 1978-01-01 1988-01-01 & 0.000 & 0.000 & 0.000 & 0.001 \\
\hline TTop Down & {$[6 \infty)$} & 1988-01-01 1998-01-01 & 0.000 & 0.000 & 0.000 & 0.052 \\
\hline TTop Down & {$[6 \infty)$} & 1998-01-01 2008-01-01 & 0.000 & 0.000 & 0.000 & 0.000 \\
\hline TTop Down & {$[6 \infty)$} & 2008-01-01 2018-01-01 & 0.000 & 0.000 & 0.000 & 0.005 \\
\hline TBot Up & [03) & 1960-01-01 1978-01-01 & 0.000 & 0.000 & 0.000 & 0.534 \\
\hline TBot Up & {$\left[\begin{array}{lll}0 & 3\end{array}\right)$} & 1978-01-01 1988-01-01 & 0.000 & 0.000 & 0.000 & 0.422 \\
\hline TBot Up & [0 3) & 1988-01-01 1998-01-01 & 0.000 & 0.000 & 0.000 & 0.431 \\
\hline
\end{tabular}


Table 7.3: US Equity In Sample Naive Event Cumulative Deviations Tests (continued)

\begin{tabular}{|c|c|c|c|c|c|c|}
\hline Type & $h_{\sigma}$ & Period & $p G_{T 1}(t)$ & $G_{T 1}(t)$ & $(1-p) G_{T 2}(t)$ & $G_{T 2}(t)$ \\
\hline TBot Up & {$[03)$} & 1998-01-01 2008-01-01 & 0.000 & 0.000 & 0.000 & 0.774 \\
\hline TBot Up & {$\left[\begin{array}{lll}0 & 3\end{array}\right)$} & 2008-01-01 2018-01-01 & 0.000 & 0.042 & 0.000 & 0.400 \\
\hline TBot Up & {$[36)$} & 1960-01-01 1978-01-01 & 0.000 & 0.000 & 0.000 & 0.206 \\
\hline TBot Up & {$[36)$} & 1978-01-01 1988-01-01 & 0.000 & 0.000 & 0.000 & 0.270 \\
\hline TBot Up & {$[36)$} & 1988-01-01 1998-01-01 & 0.000 & 0.000 & 0.000 & 0.178 \\
\hline TBot Up & {$[36)$} & 1998-01-01 2008-01-01 & 0.000 & 0.000 & 0.000 & 0.138 \\
\hline TBot Up & {$[36)$} & 2008-01-01 2018-01-01 & 0.000 & 0.073 & 0.000 & 0.376 \\
\hline TBot Up & {$[6 \infty)$} & 1960-01-01 1978-01-01 & 0.089 & 0.000 & 0.062 & 0.023 \\
\hline TBot Up & {$[6 \infty)$} & 1978-01-01 1988-01-01 & 0.081 & 0.000 & 0.037 & 0.003 \\
\hline TBot Up & {$[6 \infty)$} & 1988-01-01 1998-01-01 & 0.611 & 0.000 & 0.537 & 0.057 \\
\hline TBot Up & {$[6 \infty)$} & 1998-01-01 2008-01-01 & 0.000 & 0.000 & 0.000 & 0.000 \\
\hline TBot Up & {$[6 \infty)$} & 2008-01-01 2018-01-01 & 0.000 & 0.000 & 0.000 & 0.000 \\
\hline TBot Down & {$[03)$} & 1960-01-01 1978-01-01 & 0.000 & 0.000 & 0.000 & 0.111 \\
\hline TBot Down & {$\left[\begin{array}{lll}0 & 3\end{array}\right)$} & 1978-01-01 1988-01-01 & 0.000 & 0.000 & 0.000 & 0.330 \\
\hline TBot Down & {$\left[\begin{array}{lll}0 & 3\end{array}\right)$} & 1988-01-01 1998-01-01 & 0.000 & 0.000 & 0.000 & 0.993 \\
\hline TBot Down & {$[03)$} & 1998-01-01 2008-01-01 & 0.000 & 0.000 & 0.000 & 0.617 \\
\hline TBot Down & {$\left[\begin{array}{lll}0 & 3\end{array}\right)$} & 2008-01-01 2018-01-01 & 0.367 & 0.049 & 0.472 & 0.502 \\
\hline TBot Down & {$[36)$} & 1960-01-01 1978-01-01 & 0.000 & 0.000 & 0.000 & 0.036 \\
\hline TBot Down & {$[36)$} & 1978-01-01 1988-01-01 & 0.000 & 0.000 & 0.000 & 0.045 \\
\hline TBot Down & {$[36)$} & 1988-01-01 1998-01-01 & 0.000 & 0.000 & 0.000 & 0.104 \\
\hline TBot Down & {$[36)$} & 1998-01-01 2008-01-01 & 0.000 & 0.000 & 0.000 & 0.637 \\
\hline TBot Down & {$[36)$} & 2008-01-01 2018-01-01 & 0.000 & 0.001 & 0.000 & 0.608 \\
\hline TBot Down & {$[6 \infty)$} & 1960-01-01 1978-01-01 & 0.000 & 0.000 & 0.000 & 0.000 \\
\hline TBot Down & {$[6 \infty)$} & 1978-01-01 1988-01-01 & 0.000 & 0.000 & 0.000 & 0.003 \\
\hline TBot Down & {$[6 \infty)$} & 1988-01-01 1998-01-01 & 0.000 & 0.000 & 0.000 & 0.010 \\
\hline TBot Down & {$[6 \infty)$} & 1998-01-01 2008-01-01 & 0.000 & 0.000 & 0.000 & 0.000 \\
\hline TBot Down & {$[6 \infty)$} & 2008-01-01 2018-01-01 & 0.000 & 0.000 & 0.000 & 0.000 \\
\hline
\end{tabular}

Table 7.4: US Equity Triangle Forward Cumulative Deviations Tests

\begin{tabular}{|c|c|c|c|c|c|c|}
\hline \multirow[b]{3}{*}{ Type } & \multirow[b]{3}{*}{$h_{\sigma}$} & \multirow[b]{3}{*}{ Period } & \multicolumn{4}{|c|}{$\mathrm{p}$-value } \\
\hline & & & \multicolumn{2}{|c|}{ Win Distribution } & \multicolumn{2}{|c|}{ Loss Distribution } \\
\hline & & & $p G_{T 1}(t)$ & $G_{T 1}(t)$ & $(1-p) G_{T 2}(t)$ & $G_{T 2}(t)$ \\
\hline TTop Up & {$[03)$} & 1960-01-01 1978-01-01 & 0.026 & 0.836 & 0.009 & 0.951 \\
\hline TTop Up & {$[03)$} & 1978-01-01 1988-01-01 & 0.000 & 0.988 & 0.000 & 0.995 \\
\hline TTop Up & [0 3) & 1988-01-01 1998-01-01 & 0.000 & 0.956 & 0.000 & 0.999 \\
\hline TTop Up & {$[03)$} & 1998-01-01 2008-01-01 & 0.007 & 0.974 & 0.001 & 0.973 \\
\hline TTop Up & [03) & 2008-01-01 2018-01-01 & NA & NA & NA & NA \\
\hline TTop Up & {$[36)$} & 1960-01-01 1978-01-01 & 0.127 & 0.709 & 0.156 & 0.997 \\
\hline TTop Up & {$[36)$} & 1978-01-01 1988-01-01 & 0.202 & 0.948 & 0.068 & 0.922 \\
\hline TTop Up & {$[36)$} & 1988-01-01 1998-01-01 & 0.000 & 0.967 & 0.000 & 0.966 \\
\hline
\end{tabular}


Table 7.4: US Equity Triangle Forward Cumulative Deviations Tests (continued)

\begin{tabular}{|c|c|c|c|c|c|c|}
\hline Type & $h_{\sigma}$ & Period & $p G_{T 1}(t)$ & $G_{T 1}(t)$ & $(1-p) G_{T 2}(t)$ & $G_{T 2}(t)$ \\
\hline TTop Up & {$[36)$} & 1998-01-01 2008-01-01 & 0.003 & 0.954 & 0.001 & 0.991 \\
\hline TTop Up & {$[36)$} & 2008-01-01 2018-01-01 & NA & NA & NA & NA \\
\hline TTop Up & {$[6 \infty)$} & 1960-01-01 1978-01-01 & 0.006 & 0.933 & 0.003 & 0.980 \\
\hline TTop Up & {$[6 \infty)$} & 1978-01-01 1988-01-01 & 0.207 & 0.978 & 0.069 & 0.821 \\
\hline TTop Up & {$[6 \infty)$} & 1988-01-01 1998-01-01 & 0.000 & 0.938 & 0.000 & 0.996 \\
\hline TTop Up & {$[6 \infty)$} & 1998-01-01 2008-01-01 & 0.013 & 0.766 & 0.021 & 0.868 \\
\hline TTop Up & {$[6 \infty)$} & 2008-01-01 2018-01-01 & NA & NA & NA & NA \\
\hline TTop Down & [0 3) & 1960-01-01 1978-01-01 & 0.150 & 0.990 & 0.099 & 0.981 \\
\hline TTop Down & {$\left[\begin{array}{lll}0 & 3\end{array}\right)$} & 1978-01-01 1988-01-01 & 0.317 & 0.944 & 0.235 & 0.987 \\
\hline TTop Down & {$\left[\begin{array}{lll}0 & 3\end{array}\right)$} & 1988-01-01 1998-01-01 & 0.000 & 0.984 & 0.000 & 0.992 \\
\hline TTop Down & {$\left[\begin{array}{lll}0 & 3\end{array}\right)$} & 1998-01-01 2008-01-01 & 0.044 & 0.980 & 0.042 & 0.976 \\
\hline TTop Down & {$\left[\begin{array}{lll}0 & 3\end{array}\right)$} & 2008-01-01 2018-01-01 & NA & NA & NA & NA \\
\hline TTop Down & {$[36)$} & 1960-01-01 1978-01-01 & 0.336 & 0.838 & 0.177 & 0.964 \\
\hline TTop Down & {$[36)$} & 1978-01-01 1988-01-01 & 0.163 & 0.878 & 0.082 & 0.947 \\
\hline TTop Down & {$[36)$} & 1988-01-01 1998-01-01 & 0.007 & 0.961 & 0.003 & 0.882 \\
\hline TTop Down & {$[36)$} & 1998-01-01 2008-01-01 & 0.556 & 0.900 & 0.451 & 0.963 \\
\hline TTop Down & {$[36)$} & 2008-01-01 2018-01-01 & NA & NA & NA & NA \\
\hline TTop Down & {$[6 \infty)$} & 1960-01-01 1978-01-01 & 0.370 & 0.973 & 0.266 & 0.992 \\
\hline TTop Down & {$[6 \infty)$} & 1978-01-01 1988-01-01 & 0.004 & 0.974 & 0.001 & 0.991 \\
\hline TTop Down & {$[6 \infty)$} & 1988-01-01 1998-01-01 & 0.916 & 0.775 & 0.862 & 0.655 \\
\hline TTop Down & {$[6 \infty)$} & 1998-01-01 2008-01-01 & 0.000 & 0.936 & 0.000 & 0.978 \\
\hline TTop Down & {$[6 \infty)$} & 2008-01-01 2018-01-01 & NA & NA & NA & NA \\
\hline TBot Up & {$\left[\begin{array}{lll}0 & 3\end{array}\right)$} & 1960-01-01 1978-01-01 & 0.000 & 0.962 & 0.000 & 0.987 \\
\hline TBot Up & {$\left[\begin{array}{lll}0 & 3\end{array}\right)$} & 1978-01-01 1988-01-01 & 0.000 & 0.949 & 0.000 & 0.988 \\
\hline TBot Up & {$\left[\begin{array}{lll}0 & 3\end{array}\right)$} & 1988-01-01 1998-01-01 & 0.000 & 0.987 & 0.000 & 0.965 \\
\hline TBot Up & {$\left[\begin{array}{lll}0 & 3\end{array}\right)$} & 1998-01-01 2008-01-01 & 0.000 & 0.941 & 0.000 & 0.997 \\
\hline TBot Up & [0 3) & 2008-01-01 2018-01-01 & NA & NA & NA & NA \\
\hline TBot Up & {$[36)$} & 1960-01-01 1978-01-01 & 0.004 & 0.888 & 0.002 & 0.990 \\
\hline TBot Up & {$[36)$} & 1978-01-01 1988-01-01 & 0.404 & 0.964 & 0.245 & 0.960 \\
\hline TBot Up & {$[36)$} & 1988-01-01 1998-01-01 & 0.000 & 0.830 & 0.000 & 0.976 \\
\hline TBot Up & {$[36)$} & 1998-01-01 2008-01-01 & 0.001 & 0.974 & 0.000 & 0.964 \\
\hline TBot Up & {$[36)$} & 2008-01-01 2018-01-01 & NA & NA & NA & NA \\
\hline TBot Up & {$[6 \infty)$} & 1960-01-01 1978-01-01 & 0.102 & 0.834 & 0.130 & 0.927 \\
\hline TBot Up & {$[6 \infty)$} & 1978-01-01 1988-01-01 & 0.699 & 0.956 & 0.550 & 0.946 \\
\hline TBot Up & {$[6 \infty)$} & 1988-01-01 1998-01-01 & 0.000 & 0.880 & 0.000 & 0.942 \\
\hline TBot Up & {$[6 \infty)$} & 1998-01-01 2008-01-01 & 0.291 & 0.736 & 0.308 & 0.976 \\
\hline TBot Up & {$[6 \infty)$} & 2008-01-01 2018-01-01 & NA & NA & NA & NA \\
\hline TBot Down & [0 3) & 1960-01-01 1978-01-01 & 0.000 & 0.985 & 0.000 & 0.986 \\
\hline TBot Down & {$\left[\begin{array}{lll}0 & 3\end{array}\right)$} & 1978-01-01 1988-01-01 & 0.920 & 0.802 & 0.980 & 0.927 \\
\hline TBot Down & {$\left[\begin{array}{lll}0 & 3\end{array}\right)$} & 1988-01-01 1998-01-01 & 0.019 & 0.694 & 0.009 & 0.889 \\
\hline TBot Down & {$\left[\begin{array}{lll}0 & 3\end{array}\right)$} & 1998-01-01 2008-01-01 & 0.000 & 0.886 & 0.000 & 0.786 \\
\hline TBot Down & [0 3) & 2008-01-01 2018-01-01 & NA & NA & NA & NA \\
\hline TBot Down & {$[36)$} & 1960-01-01 1978-01-01 & 0.047 & 0.928 & 0.008 & 0.814 \\
\hline TBot Down & {$[36)$} & 1978-01-01 1988-01-01 & 0.113 & 0.974 & 0.056 & 0.892 \\
\hline
\end{tabular}


Table 7.4: US Equity Triangle Forward Cumulative Deviations Tests (continued)

\begin{tabular}{lllrrrr}
\hline Type & $h_{\sigma}$ & Period & $p G_{T 1}(t)$ & $G_{T 1}(t)$ & $(1-p) G_{T 2}(t)$ & $G_{T 2}(t)$ \\
\hline TBot Down & {$[36)$} & $1988-01-01$ 1998-01-01 & 0.359 & 0.989 & 0.312 & 0.978 \\
TBot Down & {$[36)$} & $1998-01-012008-01-01$ & 0.002 & 0.970 & 0.001 & 0.935 \\
TBot Down & {$[36)$} & $2008-01-012018-01-01$ & NA & NA & NA & NA \\
TBot Down & {$[6 \infty)$} & $1960-01-01$ 1978-01-01 & 0.358 & 0.951 & 0.236 & 0.989 \\
TBot Down & {$[6 \infty)$} & $1978-01-01$ 1988-01-01 & 0.002 & 0.991 & 0.000 & 0.947 \\
TBot Down & {$[6 \infty)$} & $1988-01-01$ 1998-01-01 & 0.423 & 0.896 & 0.413 & 0.950 \\
TBot Down & {$[6 \infty)$} & $1998-01-012008-01-01$ & 0.000 & 0.960 & 0.000 & 0.922 \\
TBot Down & {$[6 \infty)$} & $2008-01-012018-01-01$ & NA & NA & NA & NA \\
\hline
\end{tabular}



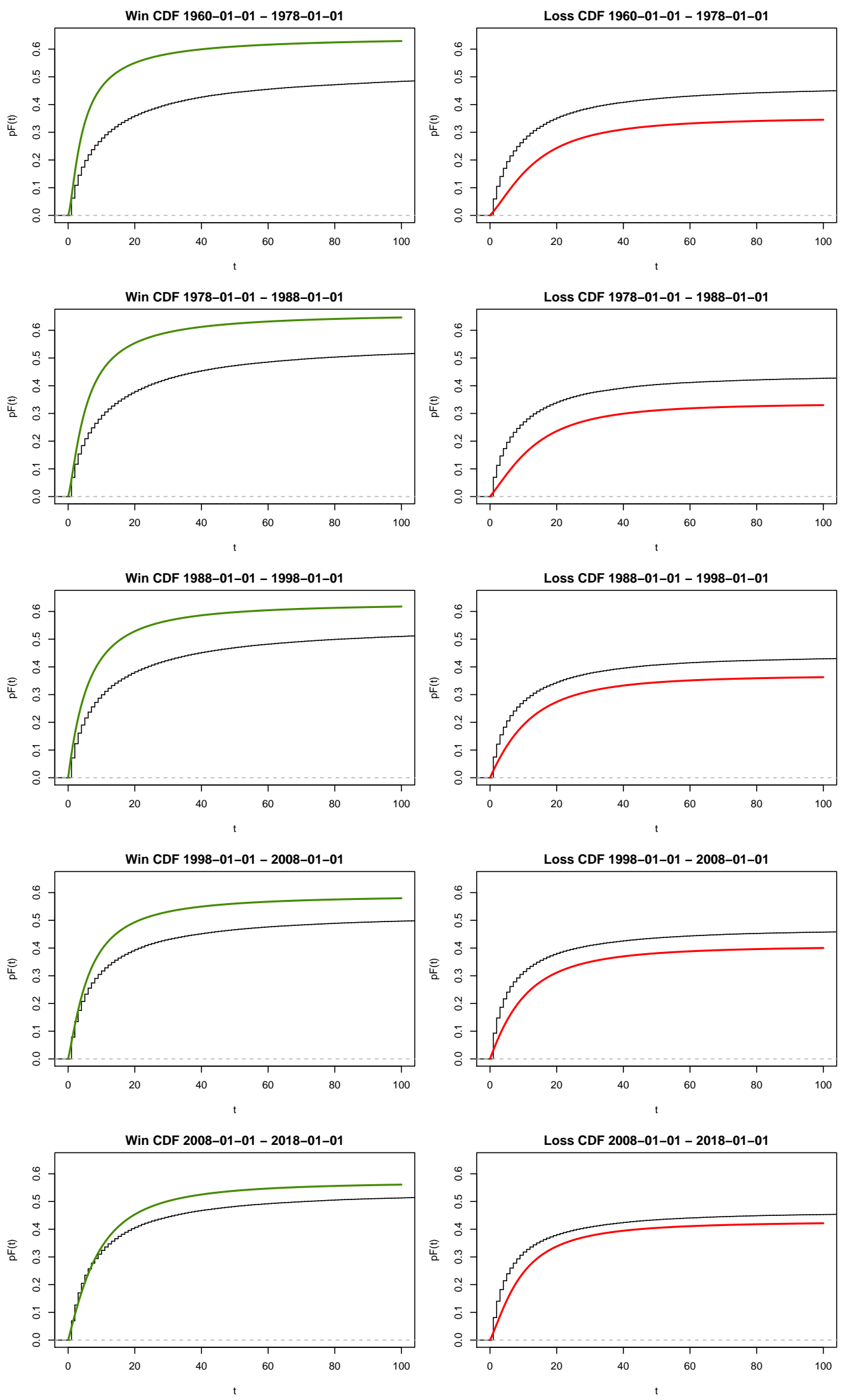

Figure 7.5: Triangle Intensity vs Naive Empirical TTop Up Profile $h_{\sigma} \in[0,3)$ 

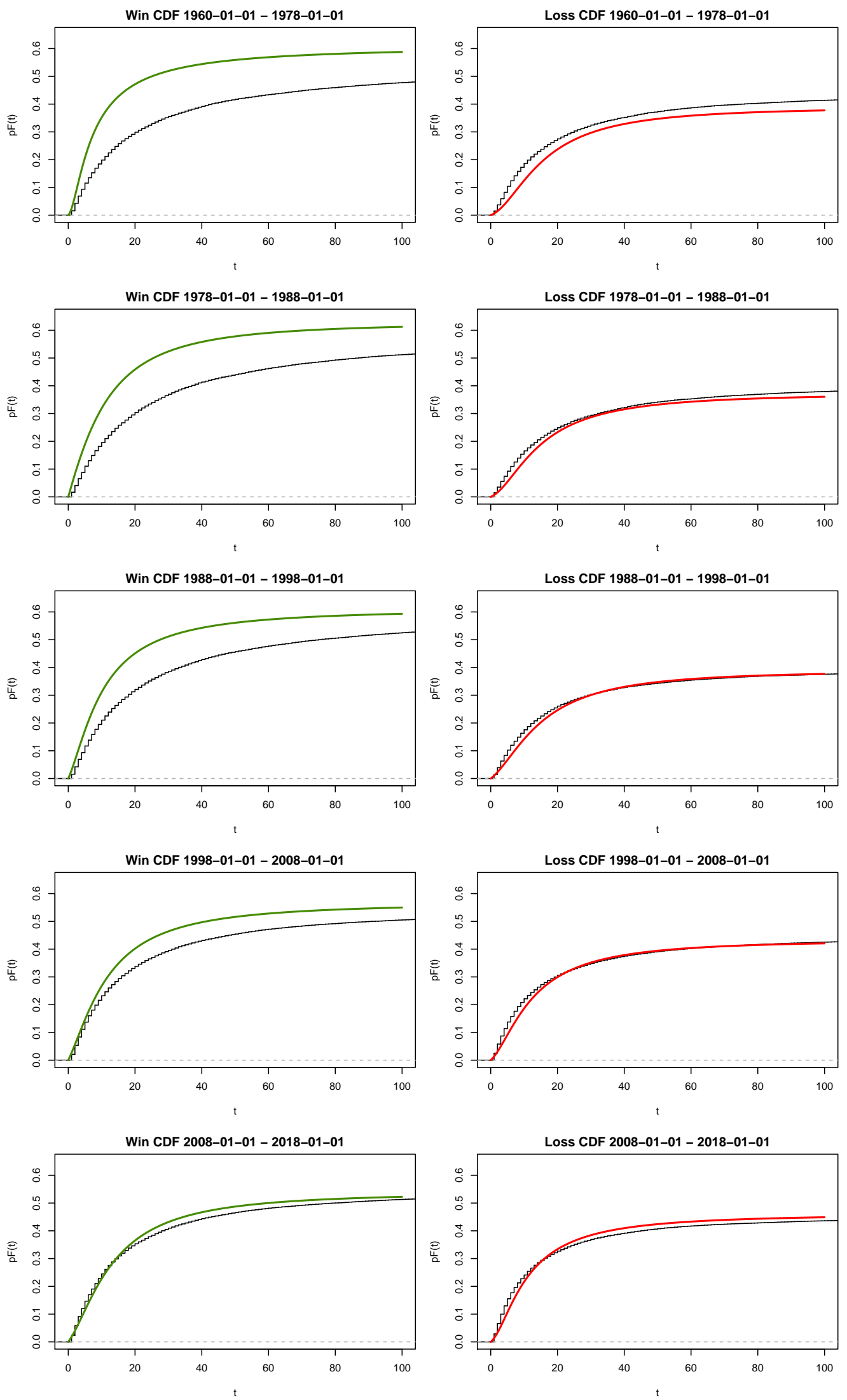

Figure 7.6: Triangle Intensity vs Naive Empirical TTop Up Profile $h_{\sigma} \in[3,6)$ 

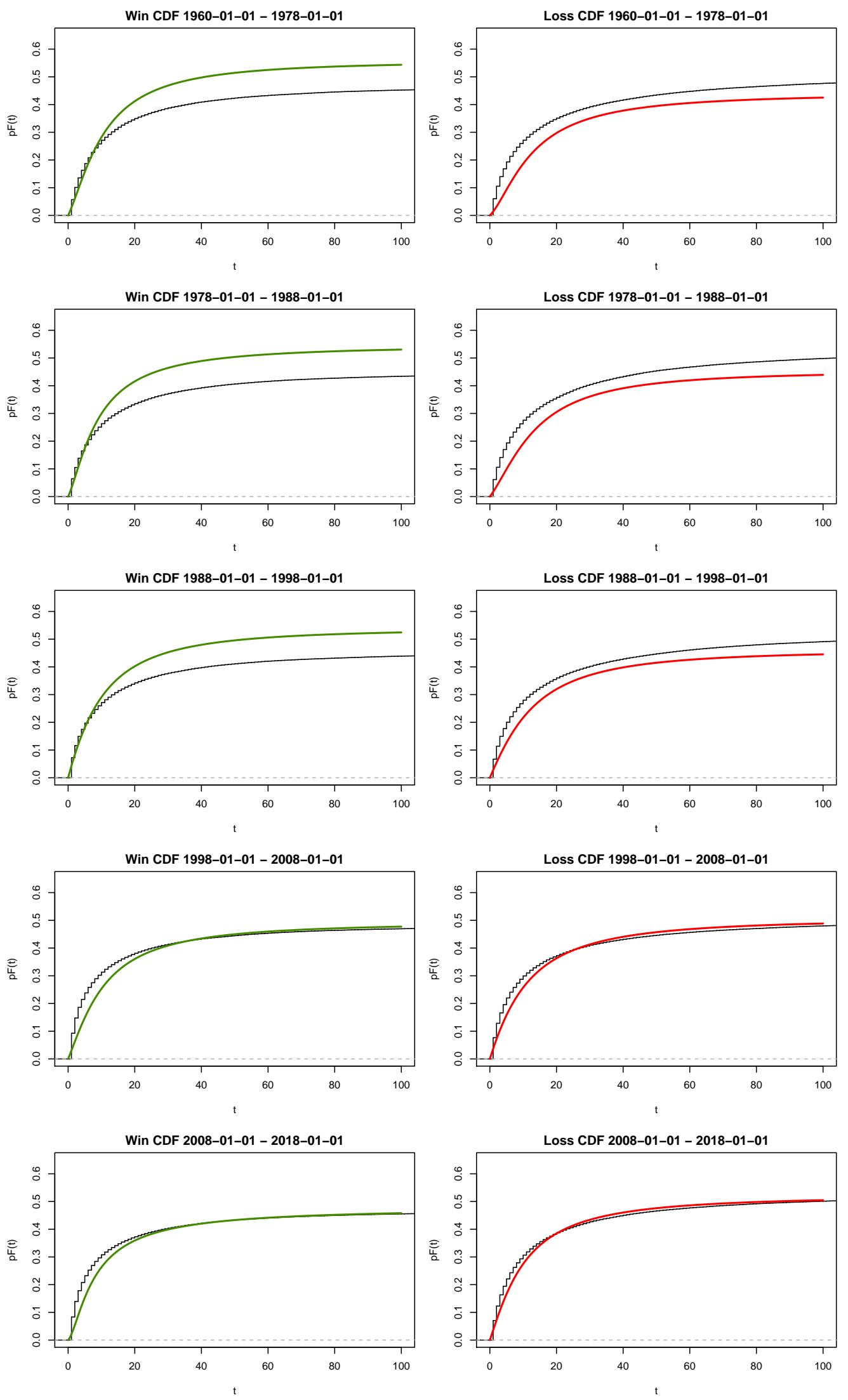

Figure 7.7: Triangle Intensity vs Naive Empirical TTop Down Profile $h_{\sigma} \in[0,3)$ 

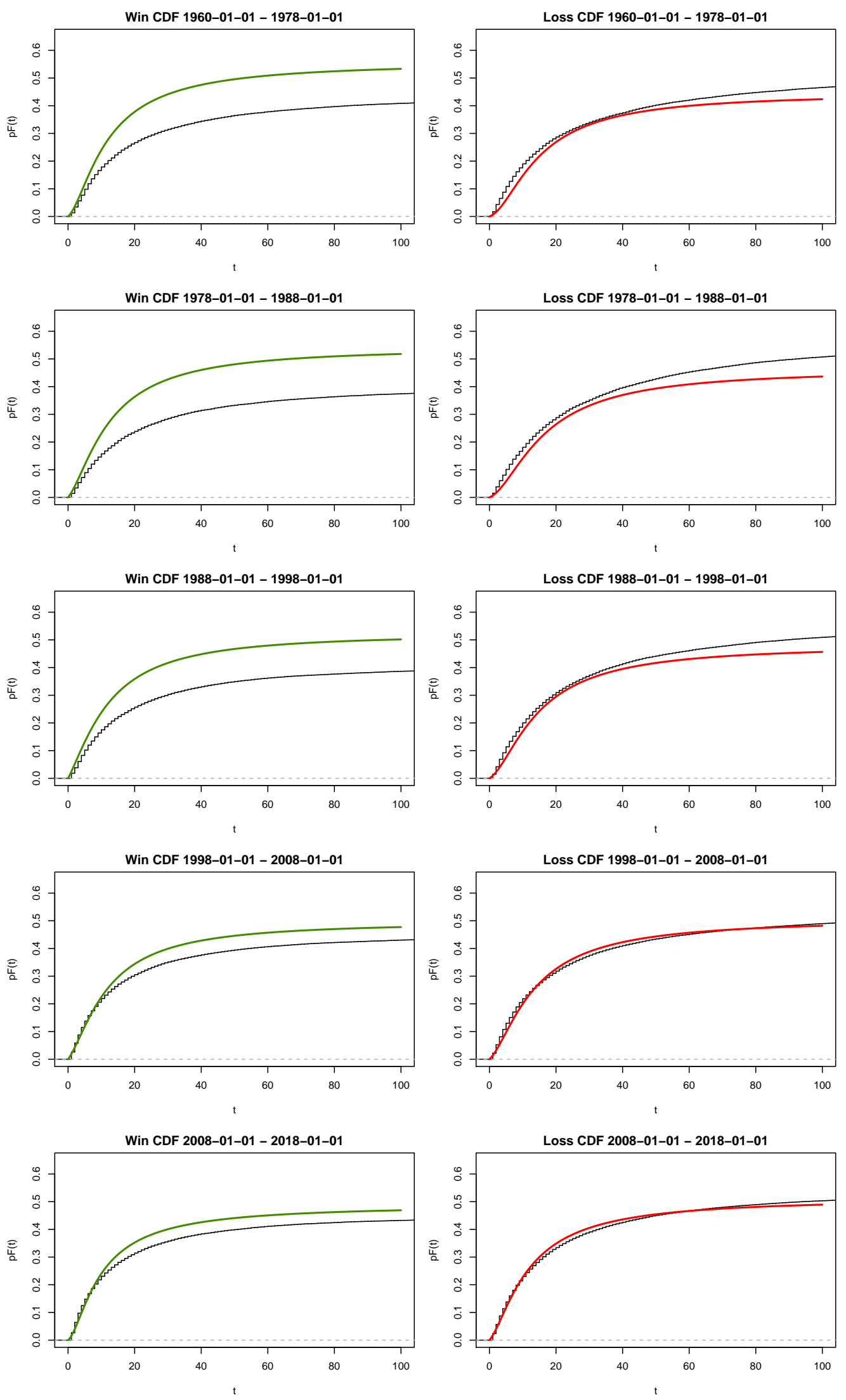

Figure 7.8: Triangle Intensity vs Naive Empirical TTop Down Profile $h_{\sigma} \in[3,6)$ 

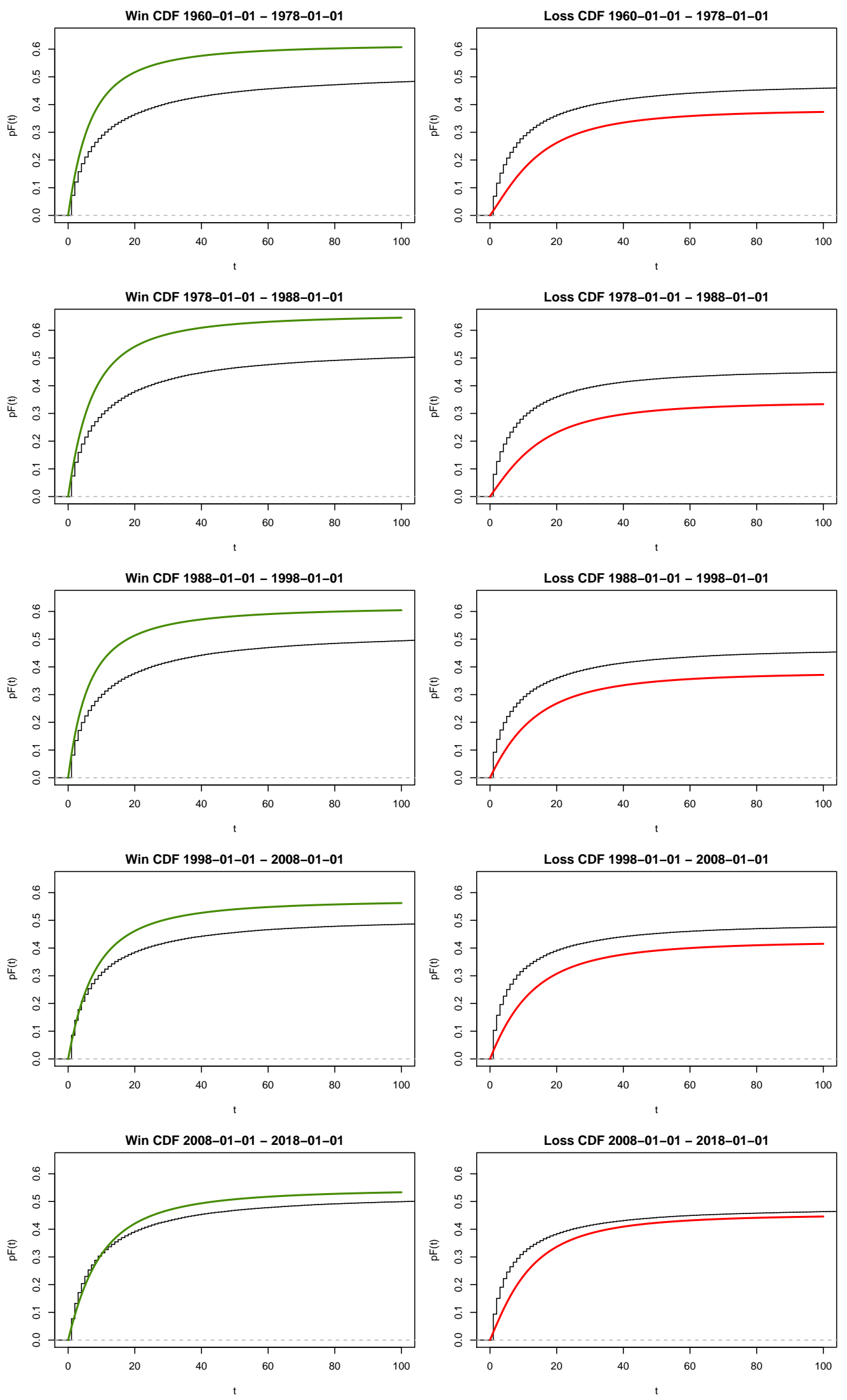

Figure 7.9: Triangle Intensity vs Naive Empirical TBot Up Profile $h_{\sigma} \in[0,3)$ 

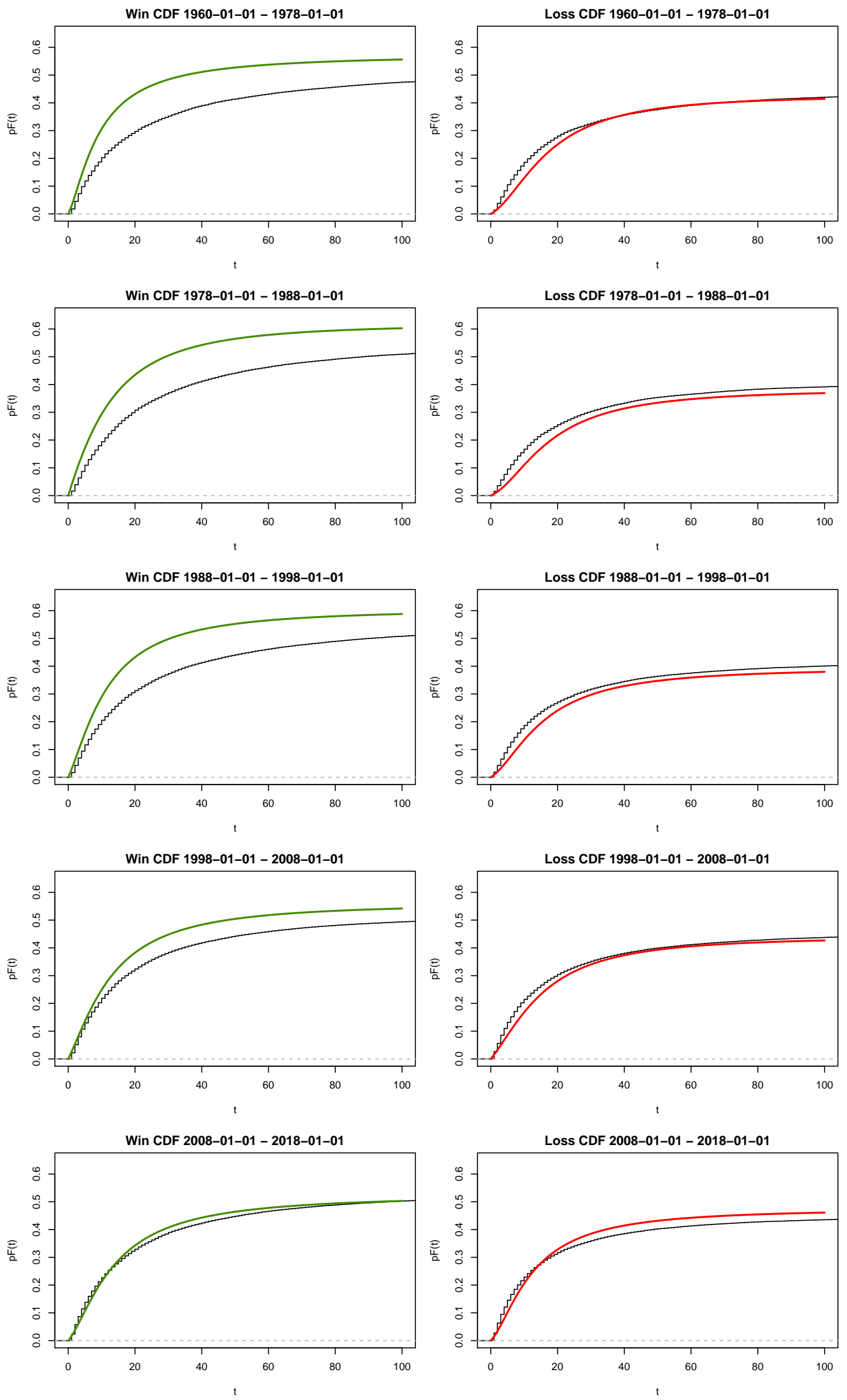

Figure 7.10: Triangle Intensity vs Naive Empirical TBot Up Profile $h_{\sigma} \in[3,6)$ 

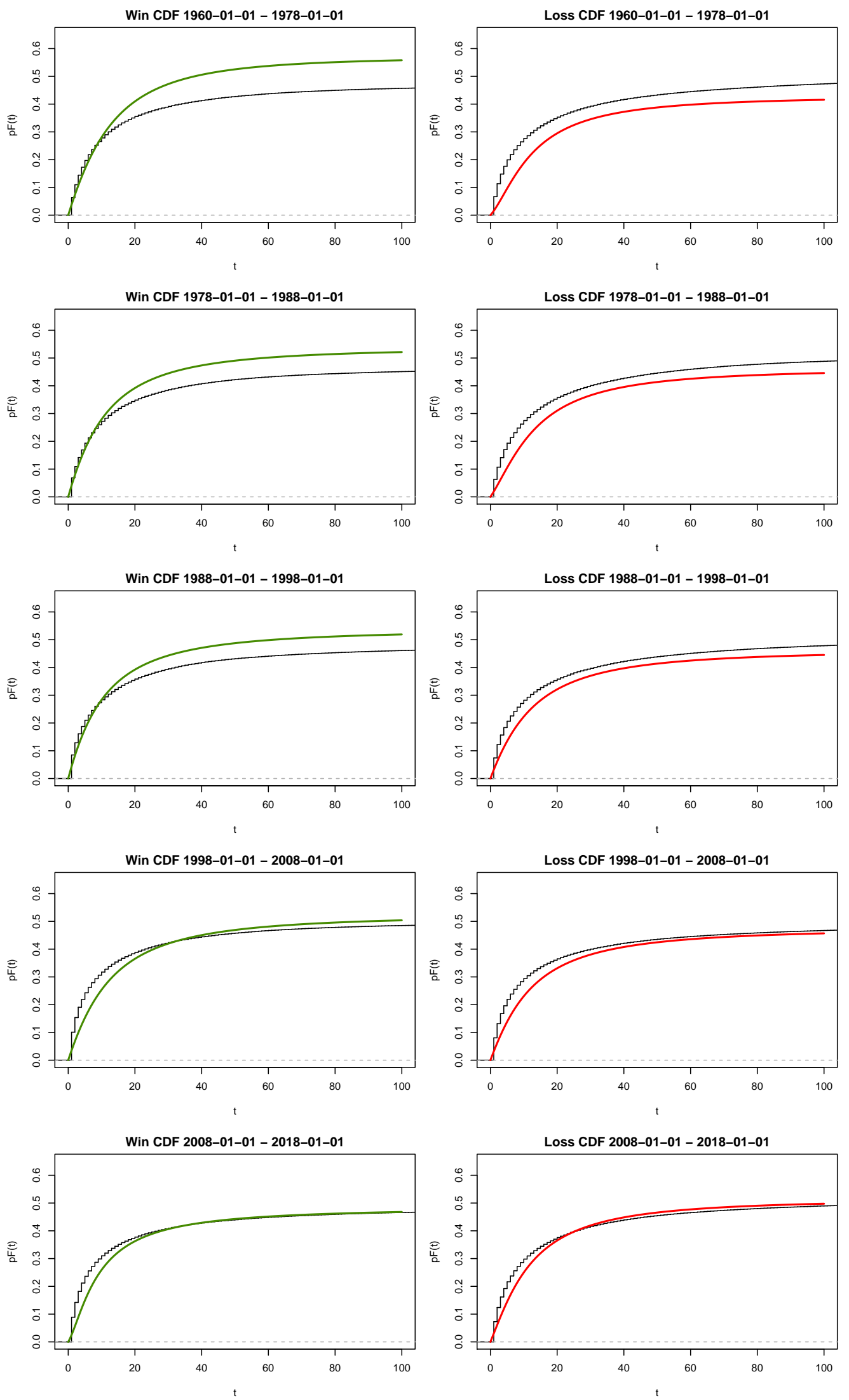

Figure 7.11: Triangle Intensity vs Naive Empirical TBot Down Profile $h_{\sigma} \in[0,3)$ 



Figure 7.12: Triangle Intensity vs Naive Empirical TBot Down Profile $h_{\sigma} \in[3,6)$ 


\subsection{Effect Size Heuristics}

Using the percentile bootstrap method, I calculate confidence intervals for mean and standard deviation across: stopping time, bet return and leveraged return. These results can be seen in Table 7.6 for Triangles and 7.7 for Naive trades where they are reported in parameter[0.025 percentile, 0.975 percentile] confidence interval form. The same Cohen's $\mathrm{d}$ is reported on both tables for convenience.

The results generally show that all types of Triangles over most $h_{\sigma}$ sections have historically had a significant contribution to positive

Table 7.5: Effect Size Heuristics Symbol Key

\begin{tabular}{|l|l|l|}
\hline Category & Measure & Symbol \\
\hline \multirow{2}{*}{ Stopping Time } & Mean Stopping Time & $\mu_{\tau}$ \\
\cline { 2 - 3 } & Standard Deviation Stopping time & $\sigma_{\tau}$ \\
\hline \multirow{2}{*}{ Bet Return } & Mean bet return & $\mu_{r_{b}}$ \\
\cline { 2 - 3 } & Standard deviation of bet return & $\sigma_{r_{b}}$ \\
\hline \multirow{3}{*}{ Leveraged Return } & Mean leveraged return & $\mu_{r_{l}}$ \\
\cline { 2 - 3 } & Standard deviation leveraged return & $\sigma_{r_{l}}$ \\
\cline { 2 - 3 } & Cohen's d & NA \\
\hline
\end{tabular}
returns, but that these have decreased over time, in some cases becoming slightly negative in later years. This is well summarised by Cohen's d showing over subsequent time sections a decreasing effect size difference normalised by volatility, up to time section 2008 to 2018 where all types can be considered to have a negligible leveraged return effect size. (Though some Triangle types become of negligible difference earlier and in later subsequent time periods this measure wavers only slightly.)

Table 7.6: US Stock Triangle Momentum Heuristics

\begin{tabular}{|c|c|c|c|c|c|c|c|c|c|}
\hline \multirow[b]{2}{*}{ Type } & \multirow[b]{2}{*}{$h_{\sigma}$} & \multirow[b]{2}{*}{ Period } & \multicolumn{2}{|c|}{ Stopping Time } & \multicolumn{2}{|c|}{ Bet Return } & \multicolumn{3}{|c|}{ Leveraged Return } \\
\hline & & & $\mu_{\tau}$ & $\sigma_{\tau}$ & $\mu_{r_{b}}$ & $\sigma_{r_{b}}$ & $\mu_{r_{l}}$ & $\sigma_{r_{l}}$ & Cohen's $d$ \\
\hline TTop Up & {$\left[\begin{array}{lll}0 & 3\end{array}\right)$} & 1960-01-01 1978-01-01 & $4.46[4.38,4.53]$ & $2.73[2.69,2.77]$ & $0.472[0.448,0.496]$ & $0.881[0.868,0.894]$ & $0.217[0.206,0.228]$ & $0.408[0.399,0.418]$ & $0.398[0.375,0.422]$ \\
\hline TTop Up & [0 3) & 1978-01-01 1988-01-01 & $4.54[4.46,4.61]$ & $2.74[2.7,2.78]$ & $0.477[0.452,0.501]$ & $0.879[0.865,0.892]$ & $0.199[0.188,0.21]$ & $0.406[0.396,0.416]$ & $0.346[0.324,0.368]$ \\
\hline TTop Up & {$\left[\begin{array}{lll}0 & 3\end{array}\right)$} & 1988-01-01 1998-01-01 & $4.21[4.14,4.28]$ & $2.79[2.75,2.82]$ & $0.371[0.349,0.393]$ & $0.928[0.919,0.937]$ & $0.176[0.164,0.188]$ & $0.49[0.481,0.499]$ & $0.304[0.282,0.327]$ \\
\hline TTop Up & [0 3) & 1998-01-01 2008-01-01 & $4.47[4.41,4.54]$ & $2.74[2.71,2.78]$ & $0.261[0.238,0.283]$ & $0.965[0.959,0.971]$ & $0.109[0.0989,0.12]$ & $0.457[0.448,0.465]$ & $0.221[0.202,0.241]$ \\
\hline TTop Up & [0 3) & 2008-01-01 2018-01-01 & $4.82[4.74,4.89]$ & $2.74[2.71,2.78]$ & $0.147[0.121,0.172]$ & $0.989[0.985,0.992]$ & $0.0611[0.05,0.0722]$ & $0.426[0.417,0.435]$ & $0.139[0.117,0.161]$ \\
\hline TTop Up & [36) & 1960-01-01 1978-01-01 & $5.18[5.03,5.32]$ & $2.66[2.59,2.73]$ & $0.443[0.393,0.491]$ & $0.896[0.87,0.919]$ & $0.154[0.138,0.172]$ & $0.308[0.291,0.325]$ & $0.376[0.326,0.426]$ \\
\hline TTop Up & [36) & 1978-01-01 1988-01-01 & $5.17[5.02,5.32]$ & $2.72[2.65,2.79]$ & $0.429[0.379,0.478]$ & $0.903[0.877,0.925]$ & $0.152[0.134,0.17]$ & $0.328[0.31,0.346]$ & $0.337[0.286,0.39]$ \\
\hline TTop Up & [36) & 1988-01-01 1998-01-01 & $5.26[5.15,5.38]$ & $2.69[2.63,2.75]$ & $0.359[0.317,0.399]$ & $0.933[0.916,0.948]$ & $0.119[0.104,0.134]$ & $0.331[0.317,0.345]$ & $0.267[0.224,0.31]$ \\
\hline
\end{tabular}




\begin{tabular}{|c|c|c|c|c|c|c|c|c|c|}
\hline Type & $h_{\sigma}$ & Period & $\mu_{\tau}$ & $\sigma_{\tau}$ & $\mu_{r_{b}}$ & $\sigma_{r_{b}}$ & $\mu_{r_{l}}$ & $\sigma_{r_{l}}$ & Cohen's $d$ \\
\hline TTop Up & [36) & 1998-01-01 2008-01-01 & $5.33[5.23,5.43]$ & $2.73[2.68,2.77]$ & $0.17[0.135,0.204]$ & $0.985[0.978,0.99]$ & $0.0627[0.0504,0.0747]$ & $0.35[0.338,0.361]$ & $0.166[0.135,0.198]$ \\
\hline TTop Up & {$[36)$} & 2008-01-01 2018-01-01 & $5.6[5.51,5.7]$ & $2.7[2.66,2.75]$ & $0.025[-0.011,0.061]$ & $0.999[0.997,1]$ & $0.0118[-0.0000801,0.0239]$ & $0.335[0.322,0.346]$ & $0.0584[0.0273,0.0892]$ \\
\hline TTop Up & {$[6 \infty)$} & 1960-01-01 1978-01-01 & $5.72[5.51,5.93]$ & $2.65[2.55,2.74]$ & $0.469[0.399,0.539]$ & $0.882[0.841,0.917]$ & $0.149[0.128,0.169]$ & $0.256[0.236,0.277]$ & $0.405[0.334,0.478]$ \\
\hline TTop Up & {$[6 \infty)$} & 1978-01-01 1988-01-01 & $5.67[5.46,5.89]$ & $2.76[2.65,2.85]$ & $0.467[0.395,0.537]$ & $0.884[0.843,0.918]$ & $0.147[0.124,0.171]$ & $0.295[0.269,0.321]$ & $0.368[0.298,0.44]$ \\
\hline TTop Up & {$[6 \infty)$} & 1988-01-01 1998-01-01 & $5.6[5.44,5.78]$ & $2.73[2.64,2.8]$ & $0.32[0.258,0.378]$ & $0.947[0.925,0.965]$ & $0.0974[0.0769,0.118]$ & $0.332[0.309,0.354]$ & $0.225[0.162,0.289]$ \\
\hline TTop Up & {$[6 \infty)$} & 1998-01-01 2008-01-01 & $5.76[5.65,5.86]$ & $2.64[2.59,2.69]$ & $0.059[0.019,0.099]$ & $0.998[0.995,0.999]$ & $0.0259[0.0134,0.0385]$ & $0.317[0.304,0.33]$ & $0.0833[0.0462,0.12]$ \\
\hline TTop Up & {$[6 \infty)$} & 2008-01-01 2018-01-01 & $5.78[5.69,5.86]$ & $2.62[2.58,2.66]$ & $0.019[-0.014,0.052]$ & $0.999[0.998,0.999]$ & $0.00413[-0.0065,0.0146]$ & $0.308[0.297,0.319]$ & $0.0515[0.0199,0.0826]$ \\
\hline TTop Down & [0 3) & 1960-01-01 1978-01-01 & $5.3[5.22,5.38]$ & $2.69[2.65,2.73]$ & $0.203[0.173,0.231]$ & $0.979[0.972,0.984]$ & $0.0704[0.0599,0.0806]$ & $0.354[0.344,0.364]$ & $0.147[0.126,0.169]$ \\
\hline TTop Down & [0 3) & 1978-01-01 1988-01-01 & $5.14[5.05,5.22]$ & $2.74[2.7,2.77]$ & $0.2[0.172,0.23]$ & $0.979[0.973,0.985]$ & $0.075[0.0635,0.0858]$ & $0.375[0.364,0.385]$ & $0.144[0.121,0.167]$ \\
\hline TTop Down & [0 3) & 1988-01-01 1998-01-01 & $4.8[4.73,4.87]$ & $2.79[2.76,2.83]$ & $0.142[0.116,0.167]$ & $0.99[0.986,0.993]$ & $0.0549[0.0434,0.0663]$ & $0.443[0.434,0.452]$ & $0.0925[0.0713,0.114]$ \\
\hline TTop Down & [0 3) & 1998-01-01 2008-01-01 & $4.86[4.78,4.92]$ & $2.76[2.72,2.79]$ & $-0.01[-0.035,0.016]$ & $1[0.999,1]$ & $-0.0186[-0.0295,-0.00806]$ & $0.428[0.419,0.437]$ & $-0.0809[-0.101,-0.0612]$ \\
\hline TTop Down & [03) & 2008-01-01 2018-01-01 & $4.94[4.86,5.01]$ & $2.72[2.68,2.76]$ & $-0.035[-0.064,-0.007]$ & $0.999[0.998,1]$ & $-0.0288[-0.0403,-0.0173]$ & $0.408[0.398,0.418]$ & $-0.0884[-0.111,-0.0655]$ \\
\hline TTop Down & [36) & 1960-01-01 1978-01-01 & $5.78[5.63,5.94]$ & $2.61[2.53,2.68]$ & $0.242[0.184,0.3]$ & $0.97[0.953,0.983]$ & $0.0722[0.0556,0.0896]$ & $0.287[0.269,0.305]$ & $0.24[0.191,0.29]$ \\
\hline TTop Down & [36) & 1978-01-01 1988-01-01 & $5.69[5.53,5.85]$ & $2.66[2.58,2.73]$ & $0.223[0.161,0.282]$ & $0.975[0.959,0.987]$ & $0.0722[0.0542,0.0903]$ & $0.296[0.277,0.314]$ & $0.233[0.182,0.285]$ \\
\hline TTop Down & [36) & 1988-01-01 1998-01-01 & $5.51[5.37,5.64]$ & $2.72[2.65,2.78]$ & $0.161[0.112,0.211]$ & $0.986[0.977,0.993]$ & $0.068[0.0506,0.0855]$ & $0.346[0.328,0.362]$ & $0.198[0.15,0.245]$ \\
\hline TTop Down & [36) & 1998-01-01 2008-01-01 & $5.42[5.31,5.52]$ & $2.64[2.59,2.69]$ & $0.055[0.015,0.094]$ & $0.998[0.995,0.999]$ & $0.021[0.00807,0.0338]$ & $0.334[0.321,0.346]$ & $0.0238[-0.00973,0.0569]$ \\
\hline TTop Down & {$[36)$} & 2008-01-01 2018-01-01 & $5.32[5.22,5.43]$ & $2.64[2.59,2.7]$ & $0.019[-0.022,0.06]$ & $0.999[0.998,1]$ & $0.00292[-0.0107,0.0167]$ & $0.34[0.327,0.353]$ & $-0.0231[-0.0589,0.0121]$ \\
\hline TTop Down & {$[6 \infty)$} & 1960-01-01 1978-01-01 & $6.1[5.88,6.32]$ & $2.59[2.48,2.69]$ & $0.292[0.212,0.37]$ & $0.955[0.927,0.976]$ & $0.0871[0.0646,0.11]$ & $0.268[0.242,0.293]$ & $0.312[0.237,0.388]$ \\
\hline TTop Down & {$[6 \infty)$} & 1978-01-01 1988-01-01 & $5.93[5.7,6.16]$ & $2.65[2.53,2.75]$ & $0.31[0.228,0.393]$ & $0.95[0.919,0.974]$ & $0.0763[0.0522,0.1]$ & $0.276[0.248,0.302]$ & $0.262[0.189,0.336]$ \\
\hline TTop Down & {$[6 \infty)$} & 1988-01-01 1998-01-01 & $5.87[5.68,6.05]$ & $2.65[2.57,2.73]$ & $0.151[0.082,0.216]$ & $0.988[0.975,0.996]$ & $0.0368[0.0162,0.0574]$ & $0.3[0.278,0.323]$ & $0.0875[0.0291,0.144]$ \\
\hline TTop Down & {$[6 \infty)$} & 1998-01-01 2008-01-01 & $5.76[5.64,5.87]$ & $2.62[2.57,2.68]$ & $0.114[0.071,0.157]$ & $0.993[0.987,0.997]$ & $0.0396[0.0267,0.0527]$ & $0.305[0.292,0.319]$ & $0.0701[0.0323,0.108]$ \\
\hline TTop Down & {$[6 \infty)$} & 2008-01-01 2018-01-01 & $5.53[5.44,5.62]$ & $2.61[2.56,2.66]$ & $0.04[0.003,0.077]$ & $0.999[0.996,0.999]$ & $0.00736[-0.00453,0.0193]$ & $0.321[0.309,0.333]$ & $-0.0145[-0.0493,0.0202]$ \\
\hline TBot Up & {$\left[\begin{array}{ll}0 & 3\end{array}\right)$} & 1960-01-01 1978-01-01 & $4.45[4.37,4.53]$ & $2.78[2.74,2.82]$ & $0.406[0.38,0.431]$ & $0.914[0.902,0.924]$ & $0.2[0.188,0.213]$ & $0.434[0.424,0.444]$ & $0.352[0.329,0.375]$ \\
\hline TBot Up & {$\left[\begin{array}{ll}0 & 3\end{array}\right]$} & 1978-01-01 1988-01-01 & 4.41[4.34,4.49] & $2.75[2.7,2.79]$ & $0.484[0.459,0.508]$ & $0.875[0.861,0.889]$ & $0.207[0.195,0.219]$ & $0.43[0.419,0.441]$ & $0.374[0.35,0.397]$ \\
\hline TBot Up & [03) & 1988-01-01 1998-01-01 & $4.13[4.07,4.2]$ & $2.84[2.8,2.88]$ & $0.377[0.355,0.398]$ & $0.926[0.917,0.935]$ & $0.182[0.169,0.194]$ & $0.516[0.507,0.525]$ & $0.318[0.296,0.34]$ \\
\hline TBot Up & [0 3) & 1998-01-01 2008-01-01 & $4.53[4.46,4.59]$ & $2.8[2.77,2.84]$ & $0.24[0.217,0.263]$ & $0.97[0.964,0.976]$ & $0.103[0.0925,0.114]$ & $0.469[0.46,0.478]$ & $0.22[0.2,0.24]$ \\
\hline TBot Up & [0 3) & 2008-01-01 2018-01-01 & $4.82[4.74,4.89]$ & $2.79[2.75,2.83]$ & $0.134[0.107,0.16]$ & $0.991[0.987,0.994]$ & $0.0621[0.0505,0.0739]$ & $0.438[0.428,0.448]$ & $0.152[0.13,0.175]$ \\
\hline TBot Up & [36) & 1960-01-01 1978-01-01 & $5.44[5.26,5.61]$ & $2.8[2.72,2.87]$ & $0.374[0.318,0.432]$ & $0.927[0.902,0.948]$ & $0.134[0.113,0.154]$ & $0.331[0.31,0.352]$ & $0.297[0.239,0.355]$ \\
\hline TBot Up & [3 6) & 1978-01-01 1988-01-01 & $5.32[5.14,5.49]$ & $2.74[2.65,2.82]$ & $0.443[0.384,0.501]$ & $0.896[0.866,0.923]$ & $0.153[0.133,0.173]$ & $0.321[0.299,0.343]$ & $0.353[0.294,0.413]$ \\
\hline TBot Up & [36) & 1988-01-01 1998-01-01 & $5.42[5.27,5.56]$ & $2.7[2.64,2.77]$ & $0.347[0.296,0.397]$ & $0.938[0.918,0.955]$ & $0.112[0.0939,0.129]$ & $0.326[0.308,0.344]$ & $0.273[0.224,0.321]$ \\
\hline
\end{tabular}




\begin{tabular}{|c|c|c|c|c|c|c|c|c|c|}
\hline Type & $h_{\sigma}$ & Period & $\mu_{\tau}$ & $\sigma_{\tau}$ & $\mu_{r_{b}}$ & $\sigma_{r_{b}}$ & $\mu_{r_{l}}$ & $\sigma_{r_{l}}$ & Cohen's $d$ \\
\hline TBot Up & {$[36)$} & 1998-01-01 2008-01-01 & $5.39[5.28,5.49]$ & $2.71[2.66,2.76]$ & $0.202[0.163,0.242]$ & $0.979[0.97,0.986]$ & $0.0583[0.0439,0.0726]$ & $0.355[0.341,0.369]$ & $0.16[0.124,0.197]$ \\
\hline TBot Up & {$[36)$} & 2008-01-01 2018-01-01 & $5.59[5.47,5.71]$ & $2.7[2.64,2.76]$ & $0.02[-0.023,0.063]$ & $0.999[0.997,0.999]$ & $0.00529[-0.00858,0.0197]$ & $0.337[0.322,0.351]$ & $0.0399[0.0034,0.0765]$ \\
\hline TBot Up & {$[6 \infty)$} & 1960-01-01 1978-01-01 & $5.72[5.48,5.95]$ & $2.69[2.58,2.79]$ & $0.403[0.322,0.483]$ & $0.915[0.876,0.946]$ & $0.128[0.104,0.152]$ & $0.284[0.256,0.311]$ & $0.327[0.251,0.405]$ \\
\hline TBot Up & {$[6 \infty)$} & 1978-01-01 1988-01-01 & $5.71[5.44,5.97]$ & $2.81[2.68,2.93]$ & $0.457[0.37,0.543]$ & $0.888[0.839,0.927]$ & $0.139[0.108,0.169]$ & $0.32[0.285,0.354]$ & $0.359[0.266,0.449]$ \\
\hline TBot Up & {$[6 \infty)$} & 1988-01-01 1998-01-01 & $5.56[5.35,5.78]$ & $2.79[2.68,2.88]$ & $0.328[0.255,0.399]$ & $0.944[0.916,0.966]$ & $0.108[0.0813,0.134]$ & $0.344[0.316,0.37]$ & $0.301[0.23,0.373]$ \\
\hline TBot Up & {$[6 \infty)$} & 1998-01-01 2008-01-01 & $5.77[5.64,5.9]$ & $2.68[2.62,2.74]$ & $0.153[0.106,0.201]$ & $0.987[0.979,0.994]$ & $0.0569[0.0418,0.0717]$ & $0.316[0.3,0.331]$ & $0.184[0.143,0.227]$ \\
\hline TBot Up & {$[6 \infty)$} & 2008-01-01 2018-01-01 & $5.9[5.79,6.02]$ & $2.65[2.6,2.7]$ & $-0.017[-0.058,0.025]$ & $0.999[0.997,0.999]$ & $0.00385[-0.00836,0.0163]$ & $0.291[0.278,0.303]$ & $0.0365[0.000313,0.0728]$ \\
\hline TBot Down & {$[03)$} & 1960-01-01 1978-01-01 & $5.22[5.15,5.29]$ & $2.81[2.77,2.84]$ & $0.191[0.166,0.216]$ & $0.981[0.976,0.985]$ & $0.0796[0.0698,0.0893]$ & $0.383[0.374,0.392]$ & $0.157[0.137,0.176]$ \\
\hline TBot Down & {$[03)$} & 1978-01-01 1988-01-01 & $5.05[4.98,5.13]$ & $2.78[2.74,2.81]$ & $0.154[0.126,0.182]$ & $0.988[0.983,0.991]$ & $0.0737[0.0626,0.0848]$ & $0.404[0.394,0.414]$ & $0.125[0.103,0.148]$ \\
\hline TBot Down & {$[03)$} & 1988-01-01 1998-01-01 & $4.6[4.53,4.66]$ & $2.85[2.82,2.88]$ & $0.116[0.093,0.138]$ & $0.993[0.99,0.995]$ & $0.0439[0.0327,0.055]$ & $0.488[0.479,0.496]$ & $0.0516[0.0308,0.0723]$ \\
\hline TBot Down & [0 3) & 1998-01-01 2008-01-01 & $4.86[4.79,4.92]$ & $2.79[2.76,2.82]$ & $0.053[0.03,0.076]$ & $0.998[0.997,0.999]$ & $0.0173[0.00732,0.0271]$ & $0.439[0.43,0.447]$ & $-0.0309[-0.0491,-0.0127]$ \\
\hline TBot Down & {$\left[\begin{array}{lll}0 & 3\end{array}\right)$} & 2008-01-01 2018-01-01 & $5[4.93,5.07]$ & $2.71[2.68,2.74]$ & $0.002[-0.024,0.029]$ & $1[0.999,1]$ & $-0.00363[-0.0145,0.00681]$ & $0.399[0.389,0.408]$ & $-0.0556[-0.0757,-0.0351]$ \\
\hline TBot Down & {$[36)$} & 1960-01-01 1978-01-01 & $5.83[5.69,5.97]$ & $2.62[2.55,2.68]$ & $0.249[0.197,0.303]$ & $0.968[0.953,0.98]$ & $0.071[0.0557,0.0861]$ & $0.282[0.266,0.298]$ & $0.228[0.182,0.273]$ \\
\hline TBot Down & {$[36)$} & 1978-01-01 1988-01-01 & $5.69[5.53,5.85]$ & $2.64[2.57,2.71]$ & $0.235[0.177,0.294]$ & $0.972[0.956,0.984]$ & $0.0805[0.0626,0.0983]$ & $0.3[0.281,0.318]$ & $0.257[0.205,0.309]$ \\
\hline TBot Down & {$[36)$} & 1988-01-01 1998-01-01 & $5.61[5.47,5.75]$ & $2.72[2.66,2.79]$ & $0.16[0.11,0.209]$ & $0.986[0.977,0.993]$ & $0.0679[0.0513,0.0849]$ & $0.329[0.312,0.346]$ & $0.191[0.145,0.239]$ \\
\hline TBot Down & [36) & 1998-01-01 2008-01-01 & $5.39[5.29,5.49]$ & $2.62[2.57,2.67]$ & $0.077[0.039,0.115]$ & $0.997[0.993,0.999]$ & $0.0294[0.0166,0.0426]$ & $0.342[0.329,0.354]$ & $0.0523[0.0194,0.0854]$ \\
\hline TBot Down & {$[36)$} & 2008-01-01 2018-01-01 & $5.33[5.22,5.43]$ & $2.6[2.55,2.65]$ & $0.081[0.042,0.12]$ & $0.996[0.992,0.999]$ & $0.0158[0.00286,0.0292]$ & $0.336[0.323,0.349]$ & $0.0049[-0.0295,0.0385]$ \\
\hline TBot Down & {$[6 \infty)$} & 1960-01-01 1978-01-01 & $6.18[5.99,6.37]$ & $2.54[2.45,2.63]$ & $0.318[0.25,0.386]$ & $0.948[0.922,0.968]$ & $0.0796[0.0621,0.097]$ & $0.238[0.221,0.256]$ & $0.264[0.206,0.325]$ \\
\hline TBot Down & {$[6 \infty)$} & 1978-01-01 1988-01-01 & $5.9[5.67,6.12]$ & $2.62[2.51,2.72]$ & $0.269[0.19,0.349]$ & $0.963[0.937,0.982]$ & $0.0862[0.0624,0.11]$ & $0.287[0.261,0.312]$ & $0.258[0.187,0.329]$ \\
\hline TBot Down & {$[6 \infty)$} & 1988-01-01 1998-01-01 & $5.78[5.6,5.98]$ & $2.71[2.62,2.8]$ & $0.105[0.035,0.175]$ & $0.994[0.984,0.999]$ & $0.0371[0.0138,0.0594]$ & $0.328[0.303,0.352]$ & $0.0385[-0.022,0.1]$ \\
\hline TBot Down & {$[6 \infty)$} & 1998-01-01 2008-01-01 & $5.85[5.73,5.96]$ & $2.59[2.54,2.64]$ & $0.087[0.043,0.132]$ & $0.995[0.991,0.998]$ & $0.0318[0.0187,0.0448]$ & $0.291[0.277,0.305]$ & $0.027[-0.00753,0.062]$ \\
\hline TBot Down & {$[6 \infty)$} & 2008-01-01 2018-01-01 & $5.7[5.6,5.8]$ & $2.57[2.53,2.62]$ & $0.092[0.056,0.129]$ & $0.995[0.991,0.998]$ & $0.0289[0.0183,0.0398]$ & $0.293[0.282,0.304]$ & $0.0313[-0.000181,0.0634]$ \\
\hline
\end{tabular}




\begin{tabular}{|c|c|c|c|c|c|c|c|c|c|}
\hline \multirow[b]{2}{*}{ Type } & \multirow[b]{2}{*}{$h_{\sigma}$} & \multirow[b]{2}{*}{ Period } & \multicolumn{2}{|c|}{ Stopping Time } & \multicolumn{2}{|c|}{ Bet Return } & \multicolumn{3}{|c|}{ Leveraged Return } \\
\hline & & & $\mu_{\tau}$ & $\sigma_{\tau}$ & $\mu_{r_{b}}$ & $\sigma_{r_{b}}$ & $\mu_{r_{l}}$ & $\sigma_{r_{l}}$ & Cohen's $d$ \\
\hline TTop Up & {$\left[\begin{array}{ll}0 & 3\end{array}\right]$} & 1960-01-01 1978-01-01 & $4.05[4.02,4.07]$ & $2.73[2.72,2.75]$ & $0.007[-0.002,0.016]$ & $0.999[0.999,0.999]$ & $0.00641[0.00151,0.0114]$ & $0.54[0.537,0.543]$ & $0.398[0.375,0.422]$ \\
\hline TTop Up & [0 3) & 1978-01-01 1988-01-01 & $3.91[3.89,3.94]$ & $2.72[2.71,2.74]$ & $0.033[0.024,0.042]$ & $0.999[0.998,0.999]$ & $0.00895[0.00392,0.0139]$ & $0.563[0.56,0.567]$ & $0.346[0.324,0.368]$ \\
\hline TTop Up & {$\left[\begin{array}{ll}0 & 3\end{array}\right]$} & 1988-01-01 1998-01-01 & $3.82[3.8,3.84]$ & $2.69[2.67,2.7]$ & $0.03[0.022,0.037]$ & $0.999[0.999,0.999]$ & $0.00434[-0.000102,0.0088]$ & $0.571[0.569,0.574]$ & $0.304[0.282,0.327]$ \\
\hline TTop Up & [0 3) & 1998-01-01 2008-01-01 & $3.68[3.66,3.69]$ & $2.64[2.63,2.65]$ & $-0.003[-0.01,0.004]$ & $0.999[0.999,1]$ & $-0.0184[-0.0224,-0.0143]$ & $0.587[0.585,0.589]$ & $0.221[0.202,0.241]$ \\
\hline TTop Up & [0 3) & 2008-01-01 2018-01-01 & $3.81[3.79,3.83]$ & $2.63[2.62,2.65]$ & $0.001[-0.007,0.009]$ & $0.999[0.999,0.999]$ & $-0.0154[-0.0199,-0.0111]$ & $0.558[0.556,0.561]$ & $0.139[0.117,0.161]$ \\
\hline TTop Up & [36) & 1960-01-01 1978-01-01 & $5.13[5.07,5.18]$ & $2.7[2.67,2.72]$ & $0.035[0.016,0.055]$ & $0.998[0.997,0.999]$ & $0.0139[0.00617,0.0215]$ & $0.382[0.375,0.389]$ & $0.376[0.326,0.426]$ \\
\hline TTop Up & [36) & 1978-01-01 1988-01-01 & $5.12[5.07,5.18]$ & $2.71[2.68,2.74]$ & $0.073[0.053,0.093]$ & $0.996[0.994,0.997]$ & $0.0226[0.0148,0.0304]$ & $0.39[0.383,0.397]$ & $0.337[0.286,0.39]$ \\
\hline TTop Up & [36) & 1988-01-01 1998-01-01 & $5.1[5.06,5.14]$ & $2.7[2.68,2.72]$ & $0.075[0.059,0.091]$ & $0.996[0.994,0.997]$ & $0.0182[0.012,0.0243]$ & $0.383[0.377,0.388]$ & $0.267[0.224,0.31]$ \\
\hline TTop Up & {$[36)$} & 1998-01-01 2008-01-01 & $4.83[4.8,4.86]$ & $2.69[2.68,2.71]$ & $0.011[0,0.023]$ & $0.999[0.999,0.999]$ & $-0.00561[-0.0101,-0.000987]$ & $0.416[0.412,0.42]$ & $0.166[0.135,0.198]$ \\
\hline TTop Up & [36) & 2008-01-01 2018-01-01 & $4.74[4.71,4.77]$ & $2.69[2.67,2.7]$ & $-0.006[-0.017,0.006]$ & $0.999[0.999,0.999]$ & $-0.0126[-0.0175,-0.0077]$ & $0.425[0.421,0.429]$ & $0.0584[0.0273,0.0892]$ \\
\hline TTop Up & {$[6 \infty)$} & 1960-01-01 1978-01-01 & $5.74[5.66,5.82]$ & $2.7[2.66,2.73]$ & $0.063[0.034,0.092]$ & $0.996[0.993,0.997]$ & $0.0204[0.011,0.0297]$ & $0.323[0.314,0.333]$ & $0.405[0.334,0.478]$ \\
\hline TTop Up & {$[6 \infty)$} & 1978-01-01 1988-01-01 & $5.5[5.42,5.58]$ & $2.76[2.72,2.8]$ & $0.078[0.048,0.108]$ & $0.995[0.992,0.997]$ & $0.014[0.00288,0.0254]$ & $0.37[0.359,0.381]$ & $0.368[0.298,0.44]$ \\
\hline TTop Up & {$[6 \infty)$} & 1988-01-01 1998-01-01 & $5.49[5.43,5.56]$ & $2.72[2.7,2.75]$ & $0.081[0.059,0.104]$ & $0.995[0.993,0.997]$ & $0.0174[0.00905,0.0258]$ & $0.359[0.351,0.367]$ & $0.225[0.162,0.289]$ \\
\hline TTop Up & {$[6 \infty)$} & 1998-01-01 2008-01-01 & $5.34[5.3,5.37]$ & $2.72[2.7,2.74]$ & $0.001[-0.012,0.014]$ & $0.998[0.998,0.998]$ & $-0.00458[-0.00937,0.000231]$ & $0.37[0.366,0.375]$ & $0.0833[0.0462,0.12]$ \\
\hline TTop Up & {$[6 \infty)$} & 2008-01-01 2018-01-01 & $5.3[5.27,5.33]$ & $2.68[2.67,2.7]$ & $-0.016[-0.026,-0.005]$ & $0.998[0.998,0.998]$ & $-0.0141[-0.018,-0.0103]$ & $0.359[0.356,0.363]$ & $0.0515[0.0199,0.0826]$ \\
\hline TTop Down & [0 3) & 1960-01-01 1978-01-01 & $4.09[4.07,4.11]$ & $2.74[2.73,2.75]$ & $-0.005[-0.014,0.004]$ & $0.999[0.999,0.999]$ & $-0.00687[-0.0115,-0.00217]$ & $0.536[0.533,0.539]$ & $0.147[0.126,0.169]$ \\
\hline TTop Down & {$\left[\begin{array}{ll}0 & 3\end{array}\right]$} & 1978-01-01 1988-01-01 & $4[3.97,4.02]$ & $2.73[2.71,2.74]$ & $-0.02[-0.029,-0.011]$ & $0.999[0.999,0.999]$ & $-0.00241[-0.00756,0.00262]$ & $0.552[0.549,0.555]$ & $0.144[0.121,0.167]$ \\
\hline TTop Down & {$\left[\begin{array}{ll}0 & 3\end{array}\right]$} & 1988-01-01 1998-01-01 & $3.86[3.84,3.88]$ & $2.71[2.7,2.72]$ & $-0.012[-0.019,-0.004]$ & $0.999[0.999,0.999]$ & $0.00312[-0.00136,0.00758]$ & $0.57[0.567,0.573]$ & $0.0925[0.0713,0.114]$ \\
\hline TTop Down & [03) & 1998-01-01 2008-01-01 & $3.66[3.64,3.68]$ & $2.65[2.64,2.66]$ & $0.027[0.02,0.034]$ & $0.999[0.999,0.999]$ & $0.0284[0.0242,0.0325]$ & $0.591[0.589,0.594]$ & $-0.0809[-0.101,-0.0612]$ \\
\hline TTop Down & [0 3) & 2008-01-01 2018-01-01 & $3.77[3.75,3.8]$ & $2.65[2.64,2.66]$ & $0.01[0.001,0.018]$ & $0.999[0.999,0.999]$ & $0.0206[0.0157,0.0255]$ & $0.571[0.568,0.574]$ & $-0.0884[-0.111,-0.0655]$ \\
\hline TTop Down & {$[36)$} & 1960-01-01 1978-01-01 & $5.15[5.1,5.21]$ & $2.71[2.69,2.74]$ & $-0.024[-0.044,-0.004]$ & $0.998[0.998,0.999]$ & $-0.0179[-0.0257,-0.0102]$ & $0.384[0.377,0.391]$ & $0.24[0.191,0.29]$ \\
\hline TTop Down & [36) & 1978-01-01 1988-01-01 & $5.12[5.06,5.17]$ & $2.72[2.69,2.75]$ & $-0.057[-0.078,-0.036]$ & $0.997[0.996,0.998]$ & $-0.0169[-0.0252,-0.00859]$ & $0.391[0.384,0.399]$ & $0.233[0.182,0.285]$ \\
\hline TTop Down & [36) & 1988-01-01 1998-01-01 & $5.05[5.01,5.1]$ & $2.71[2.68,2.73]$ & $-0.046[-0.063,-0.029]$ & $0.998[0.997,0.998]$ & $-0.00887[-0.0155,-0.0022]$ & $0.394[0.388,0.4]$ & $0.198[0.15,0.245]$ \\
\hline TTop Down & {$[36)$} & 1998-01-01 2008-01-01 & $4.8[4.76,4.83]$ & $2.71[2.69,2.72]$ & $0.009[-0.003,0.021]$ & $0.999[0.999,0.999]$ & $0.0111[0.00597,0.0163]$ & $0.423[0.419,0.428]$ & $0.0238[-0.00973,0.0569]$ \\
\hline TTop Down & [3 6) & 2008-01-01 2018-01-01 & $4.73[4.69,4.76]$ & $2.7[2.68,2.72]$ & $0.014[0.001,0.028]$ & $0.999[0.998,0.999]$ & $0.0126[0.00703,0.0184]$ & $0.428[0.423,0.432]$ & $-0.0231[-0.0589,0.0121]$ \\
\hline TTop Down & {$[6 \infty)$} & 1960-01-01 1978-01-01 & $5.56[5.48,5.64]$ & $2.68[2.64,2.72]$ & $-0.042[-0.071,-0.012]$ & $0.997[0.996,0.998]$ & $-0.0177[-0.0281,-0.00735]$ & $0.343[0.333,0.353]$ & $0.312[0.237,0.388]$ \\
\hline TTop Down & {$[6 \infty)$} & 1978-01-01 1988-01-01 & $5.36[5.28,5.45]$ & $2.77[2.73,2.81]$ & $-0.087[-0.117,-0.055]$ & $0.995[0.991,0.997]$ & $-0.0217[-0.0333,-0.00988]$ & $0.384[0.372,0.395]$ & $0.262[0.189,0.336]$ \\
\hline TTop Down & {$[6 \infty)$} & 1988-01-01 1998-01-01 & $5.26[5.19,5.33]$ & $2.8[2.77,2.83]$ & $-0.033[-0.058,-0.009]$ & $0.998[0.997,0.999]$ & $0.00276[-0.00695,0.0124]$ & $0.4[0.391,0.408]$ & $0.0875[0.0291,0.144]$ \\
\hline
\end{tabular}




\begin{tabular}{|c|c|c|c|c|c|c|c|c|c|}
\hline Type & $h_{\sigma}$ & Period & $\mu_{\tau}$ & $\sigma_{\tau}$ & $\mu_{r_{b}}$ & $\sigma_{r_{b}}$ & $\mu_{r_{l}}$ & $\sigma_{r_{l}}$ & Cohen's $d$ \\
\hline TTop Down & {$[6 \infty)$} & 1998-01-01 2008-01-01 & $5.19[5.15,5.23]$ & $2.76[2.74,2.78]$ & $0.02[0.006,0.034]$ & $0.998[0.998,0.998]$ & $0.0124[0.00671,0.0179]$ & $0.396[0.391,0.401]$ & $0.0701[0.0323,0.108]$ \\
\hline TTop Down & {$[6 \infty)$} & 2008-01-01 2018-01-01 & $5.23[5.19,5.26]$ & $2.69[2.68,2.71]$ & $0.013[0,0.026]$ & $0.998[0.998,0.998]$ & $0.0126[0.00784,0.0174]$ & $0.37[0.365,0.374]$ & $-0.0145[-0.0493,0.0202]$ \\
\hline TBot Up & {$[03)$} & 1960-01-01 1978-01-01 & $3.91[3.88,3.93]$ & $2.72[2.7,2.73]$ & $0.002[-0.007,0.011]$ & $0.999[0.999,0.999]$ & $0.0059[0.000968,0.0108]$ & $0.563[0.56,0.566]$ & $0.352[0.329,0.375]$ \\
\hline TBot Up & [03) & 1978-01-01 1988-01-01 & $3.83[3.8,3.85]$ & $2.71[2.7,2.73]$ & $0.008[-0.001,0.017]$ & $0.999[0.999,1]$ & $-0.00463[-0.00989,0.000663]$ & $0.578[0.574,0.581]$ & $0.374[0.35,0.397]$ \\
\hline TBot Up & [0 3) & 1988-01-01 1998-01-01 & $3.64[3.62,3.66]$ & $2.67[2.66,2.68]$ & $0.011[0.004,0.019]$ & $0.999[0.999,1]$ & $-0.00813[-0.0127,-0.0036]$ & $0.603[0.601,0.606]$ & $0.318[0.296,0.34]$ \\
\hline TBot Up & [03) & 1998-01-01 2008-01-01 & $3.59[3.57,3.61]$ & $2.64[2.63,2.66]$ & $-0.026[-0.032,-0.019]$ & $0.999[0.999,0.999]$ & $-0.0279[-0.032,-0.0237]$ & $0.605[0.603,0.607]$ & $0.22[0.2,0.24]$ \\
\hline TBot Up & [0 3) & 2008-01-01 2018-01-01 & $3.67[3.65,3.69]$ & $2.63[2.62,2.65]$ & $-0.012[-0.02,-0.004]$ & $0.999[0.999,1]$ & $-0.0255[-0.0303,-0.0207]$ & $0.586[0.583,0.589]$ & $0.152[0.13,0.175]$ \\
\hline TBot Up & {$[36)$} & 1960-01-01 1978-01-01 & $5.08[5.02,5.14]$ & $2.72[2.7,2.75]$ & $0.037[0.016,0.058]$ & $0.998[0.997,0.999]$ & $0.0201[0.0118,0.0283]$ & $0.387[0.38,0.395]$ & $0.297[0.239,0.355]$ \\
\hline TBot Up & [36) & 1978-01-01 1988-01-01 & $5.12[5.06,5.18]$ & $2.71[2.68,2.74]$ & $0.067[0.044,0.089]$ & $0.997[0.995,0.998]$ & $0.0175[0.0088,0.0261]$ & $0.39[0.382,0.398]$ & $0.353[0.294,0.413]$ \\
\hline TBot Up & [36) & 1988-01-01 1998-01-01 & $5.05[5,5.1]$ & $2.71[2.68,2.73]$ & $0.031[0.014,0.049]$ & $0.998[0.998,0.999]$ & $0.00517[-0.00183,0.0121]$ & $0.396[0.39,0.402]$ & $0.273[0.224,0.321]$ \\
\hline TBot Up & {$[36)$} & 1998-01-01 2008-01-01 & $4.82[4.79,4.86]$ & $2.72[2.7,2.74]$ & $-0.007[-0.02,0.006]$ & $0.999[0.999,0.999]$ & $-0.00875[-0.0142,-0.00338]$ & $0.425[0.42,0.429]$ & $0.16[0.124,0.197]$ \\
\hline TBot Up & {$[36)$} & 2008-01-01 2018-01-01 & $4.72[4.68,4.75]$ & $2.7[2.68,2.71]$ & $-0.013[-0.026,0.001]$ & $0.999[0.999,0.999]$ & $-0.0115[-0.0174,-0.00547]$ & $0.429[0.424,0.434]$ & $0.0399[0.0034,0.0765]$ \\
\hline TBot Up & {$[6 \infty)$} & 1960-01-01 1978-01-01 & $5.62[5.54,5.71]$ & $2.76[2.72,2.8]$ & $0.04[0.009,0.071]$ & $0.997[0.996,0.998]$ & $0.0145[0.00366,0.0256]$ & $0.354[0.343,0.365]$ & $0.327[0.251,0.405]$ \\
\hline TBot Up & {$[6 \infty)$} & 1978-01-01 1988-01-01 & $5.39[5.3,5.48]$ & $2.78[2.74,2.82]$ & $0.041[0.008,0.074]$ & $0.997[0.996,0.998]$ & $0.00627[-0.00602,0.0187]$ & $0.374[0.362,0.386]$ & $0.359[0.266,0.449]$ \\
\hline TBot Up & {$[6 \infty)$} & 1988-01-01 1998-01-01 & $5.23[5.15,5.3]$ & $2.8[2.76,2.83]$ & $0.019[-0.006,0.045]$ & $0.998[0.997,0.999]$ & $-0.0124[-0.0229,-0.00164]$ & $0.404[0.395,0.413]$ & $0.301[0.23,0.373]$ \\
\hline TBot Up & {$[6 \infty)$} & 1998-01-01 2008-01-01 & $5.2[5.16,5.25]$ & $2.77[2.75,2.79]$ & $-0.021[-0.036,-0.006]$ & $0.998[0.998,0.998]$ & $-0.015[-0.0208,-0.00921]$ & $0.397[0.391,0.402]$ & $0.184[0.143,0.227]$ \\
\hline TBot Up & {$[6 \infty)$} & 2008-01-01 2018-01-01 & $5.32[5.28,5.36]$ & $2.71[2.7,2.73]$ & $-0.007[-0.02,0.007]$ & $0.998[0.998,0.999]$ & $-0.00937[-0.0143,-0.00419]$ & $0.369[0.365,0.374]$ & $0.0365[0.000313,0.0728]$ \\
\hline TBot Down & {$\left[\begin{array}{lll}0 & 3\end{array}\right)$} & 1960-01-01 1978-01-01 & $3.97[3.95,3.99]$ & $2.74[2.72,2.75]$ & $0[-0.008,0.008]$ & $0.999[0.999,0.999]$ & $-0.00525[-0.00941,-0.00108]$ & $0.554[0.551,0.556]$ & $0.157[0.137,0.176]$ \\
\hline TBot Down & {$\left[\begin{array}{ll}0 & 3\end{array}\right)$} & 1978-01-01 1988-01-01 & $3.98[3.96,4]$ & $2.73[2.72,2.74]$ & $-0.003[-0.011,0.006]$ & $0.999[0.999,0.999]$ & $0.00528[0.000635,0.01]$ & $0.557[0.554,0.56]$ & $0.125[0.103,0.148]$ \\
\hline TBot Down & [03) & 1988-01-01 1998-01-01 & $3.73[3.71,3.75]$ & $2.7[2.69,2.71]$ & $0.006[-0.001,0.014]$ & $0.999[0.999,1]$ & $0.0137[0.00947,0.018]$ & $0.593[0.591,0.596]$ & $0.0516[0.0308,0.0723]$ \\
\hline TBot Down & {$\left[\begin{array}{ll}0 & 3\end{array}\right)$} & 1998-01-01 2008-01-01 & $3.59[3.57,3.61]$ & $2.65[2.64,2.66]$ & $0.04[0.034,0.046]$ & $0.999[0.998,0.999]$ & $0.0357[0.0319,0.0396]$ & $0.606[0.604,0.608]$ & $-0.0309[-0.0491,-0.0127]$ \\
\hline TBot Down & {$[03)$} & 2008-01-01 2018-01-01 & $3.71[3.69,3.73]$ & $2.65[2.63,2.66]$ & $0.023[0.016,0.031]$ & $0.999[0.999,0.999]$ & $0.028[0.0235,0.0326]$ & $0.582[0.58,0.585]$ & $-0.0556[-0.0757,-0.0351]$ \\
\hline TBot Down & {$[36)$} & 1960-01-01 1978-01-01 & $5.12[5.07,5.17]$ & $2.71[2.69,2.74]$ & $-0.044[-0.063,-0.026]$ & $0.998[0.997,0.998]$ & $-0.014[-0.0209,-0.00699]$ & $0.382[0.376,0.388]$ & $0.228[0.182,0.273]$ \\
\hline TBot Down & {$[36)$} & 1978-01-01 1988-01-01 & $5.17[5.12,5.23]$ & $2.71[2.69,2.74]$ & $-0.051[-0.07,-0.031]$ & $0.997[0.996,0.998]$ & $-0.0161[-0.0235,-0.00844]$ & $0.383[0.376,0.389]$ & $0.257[0.205,0.309]$ \\
\hline TBot Down & {$[36)$} & 1988-01-01 1998-01-01 & $5.07[5.03,5.12]$ & $2.71[2.69,2.74]$ & $-0.04[-0.057,-0.023]$ & $0.998[0.997,0.998]$ & $-0.00627[-0.0128,0.000345]$ & $0.394[0.388,0.4]$ & $0.191[0.145,0.239]$ \\
\hline TBot Down & {$[36)$} & 1998-01-01 2008-01-01 & $4.79[4.75,4.82]$ & $2.71[2.7,2.73]$ & $-0.001[-0.013,0.011]$ & $0.999[0.999,0.999]$ & $0.00732[0.00221,0.0125]$ & $0.429[0.425,0.433]$ & $0.0523[0.0194,0.0854]$ \\
\hline TBot Down & {$[36)$} & 2008-01-01 2018-01-01 & $4.72[4.68,4.76]$ & $2.7[2.68,2.72]$ & $0.017[0.003,0.03]$ & $0.999[0.998,0.999]$ & $0.0137[0.00797,0.0194]$ & $0.434[0.429,0.439]$ & $0.0049[-0.0295,0.0385]$ \\
\hline TBot Down & {$[6 \infty)$} & 1960-01-01 1978-01-01 & $5.63[5.56,5.7]$ & $2.7[2.67,2.73]$ & $0.002[-0.025,0.028]$ & $0.998[0.997,0.998]$ & $-0.00738[-0.0164,0.00169]$ & $0.339[0.33,0.348]$ & $0.264[0.206,0.325]$ \\
\hline TBot Down & {$[6 \infty)$} & 1978-01-01 1988-01-01 & $5.29[5.22,5.37]$ & $2.75[2.71,2.78]$ & $-0.052[-0.08,-0.025]$ & $0.997[0.995,0.998]$ & $-0.0107[-0.0213,0.0000137]$ & $0.384[0.374,0.394]$ & $0.258[0.187,0.329]$ \\
\hline TBot Down & {$[6 \infty)$} & 1988-01-01 1998-01-01 & $5.12[5.05,5.18]$ & $2.81[2.78,2.84]$ & $0.022[-0.001,0.045]$ & $0.998[0.997,0.999]$ & $0.0213[0.0117,0.0309]$ & $0.418[0.41,0.427]$ & $0.0385[-0.022,0.1]$ \\
\hline
\end{tabular}


Table 7.7: US Equity Naive Event Heuristics (continued)

\begin{tabular}{|c|c|c|c|c|c|c|c|c|c|}
\hline Type & $h_{\sigma}$ & Period & $\mu_{\tau}$ & $\sigma_{\tau}$ & $\mu_{r_{b}}$ & $\sigma_{r_{b}}$ & $\mu_{r_{l}}$ & $\sigma_{r_{l}}$ & Cohen's $d$ \\
\hline TBot Down & {$[6 \infty)$} & 1998-01-01 2008-01-01 & $5.09[5.06,5.13]$ & $2.78[2.77,2.8]$ & $0.034[0.02,0.048]$ & $0.998[0.997,0.998]$ & $0.0209[0.0153,0.0266]$ & $0.415[0.41,0.42]$ & $0.027[-0.00753,0.062]$ \\
\hline TBot Down & {$[6 \infty)$} & 2008-01-01 2018-01-01 & $5.2[5.16,5.23]$ & $2.72[2.7,2.73]$ & $0.034[0.021,0.048]$ & $0.998[0.997,0.998]$ & $0.0172[0.0122,0.0222]$ & $0.384[0.38,0.389]$ & $0.0313[-0.000181,0.0634]$ \\
\hline
\end{tabular}




\section{Chapter 8}

\section{Discussion}

This research has aimed to produce a complete end-to end study of how returns are evaluated for trades initiated from Triangles, while also gaining a deeper exploratory understanding of the phenomenon than other studies. Had this thesis been more focused on one aspect, for example a sole focus on modelling the arrival time results, it would have been easier to gain greater depth. Also, due to background literature using weak research methodology, a significant amount of work needed to be done to develop ideas from scratch, and so numerous procedures had to be invented, then simplified to produce robust results. Much of this discussion covers aspects which were considered but which I have not been able to pursue or show in previous chapters; however, I start by discussing how well the results cover the research goals.

The results are transparent, significantly more so than other related studies partly because other studies focus on p-value tests and do not report effect size, for example absolute return amounts. Using the Intensity GEV sub-distribution comparison and Cohen's $d$ it is clear that there has been an impact of Triangles on returns and that this has decreased over time. This clarifies the often confusing results of the previous literature from which I could draw no definitive conclusions. The previous literature discussed in Section 2.2.2 had been conflicting, sometimes stating that such patterns have had an effect and sometimes not, often using tests which despite being fairly standard were not clear in their implementation. So despite having to make numerous simplifications to cover the material, this study has overall managed to show the effect in a way that is transparent and fix issues with previous work.

However while the Intensity model fits well and certainly shows interesting differences between the Triangle and Naive cases, this method requires more thought to fully uncover these results. The test results of the Intensity model against the Naive case show that the win $\tau$ distributions are consistently different and yet the loss distributions are not. These results are consistent, but they do not measure clearly how different the sub-distributions are since these tests usually quickly converge to zero. I tried numerous 
methods to measure this concluding that the differences were best understood visually as sub-distribution plots as seen in Figures 7.5 to 7.12. The sub-distribution plots show the variation of arrival time and probability in a clearly understandable way, simply as thes y show the difference between plotted CDF lines. While I could have displayed various other statistics and tests, none of these seemed to provide significant additional useful information over the clarity of these plots, and in many cases the numbers they produce are obscure and difficult to interpret. It may require considerable work to develop a new goodness of fit measure or a measure that indicates how advantageous the Intensity model is for trading.

Also, unfortunately the Cumulative Deviations p-value based testing is of an unknown accuracy. I suspect that measurement differences and the large sample size will cause there to be inaccuracies with the testing (a well known problem) of Triangle and Naive models, but I do not know by how much. The way I use the Cumulative Deviations Test $\mathrm{p}$-values is as a confirmatory measure of goodness fit, rather than a strict discrete null hypothesis test. By repeatedly confirming the results, this should help to overcome testing inaccuracy or consistency issues, since this alleviates issues with a single test and requirements of absolute accuracy to a defined level of significance (often considered a poor research technique and commonly criticized in the statistics literature). Fortunately the Effect Heuristics are not affected adversely by sample size and the effect difference that these results show is quite clear, which confirms the Intensity GEV results.

It would be interesting to use a more data science based methodology to understand what affects the change in probability of winning and arrival times, potentially using varied exploratory methods and multiple investigations of what affects the Triangle results. Breiman (2001a) comments well on this, relating how focusing less on distribution theory and more on predictability and newer methods, creates an entirely different culture of research. For example, in an initial first effort at taking into account more information, news events should be taken into account. It is likely that with certain news announcements prices will show some congestion, perhaps as market participants avoid trading to wait for the news announcement; then when the news announcement is made the price of the security will likely show larger moves than normal. However in keeping with research methodology advice, I decided to very carefully focus on relative height changes and the competitive advantage of timing without considering numerous details, this within the context of two clearly defined statistical frameworks. This makes the results very clear and leaves little room for error, but lacks the wider understanding that the use of multiple variables or more widely creative methods could provide. It seems better at this early exploratory stage of research to carefully confirm the results within a focused framework.

Very little effort has been put into more exact forward testing and creation of prediction measurement aspects, and it would be interesting to understand how well the predictive 
capacity has held over time. The Intensity GEV model fits surprisingly well and with some effort it is likely possible to account for covariates within the model to more carefully create a forward comparable model, for example taking into account systematic market effects, which should sharpen the test for forward prediction.

It is partly disappointing to not make more use of the fitted Intensity GEV model formula, perhaps to show how well this can be used to predict the distribution of returns, but such manipulations seem superfluous without further effort spent on modelling. The model fits are good and what these do show is that alternative models can be used to model returns particularly effectively, as compared with assumptions of the log normality of periodic returns, since typically log normal returns do not fit stock returns well. A problem with typical log normal distribution fits of periodic stock returns are that the empirical tails are fat, and hence usually do not match the distribution. The solution to invert the problem using price levels means that the previous periodic return distribution fat tails are perhaps now able to be modelled. However further work would need to be done to make the approach more accessible, as currently it is in a fairly raw form that requires more development.

\subsection{Alternative Result Evaluation Models}

The failure of the Structural approach had been a surprise, which fortunately spurred more practical alternative methods, mainly converging on the Intensity GEV and Effect Heuristics; other ideas were considered, some of which have potential for better results.

The Structural approach gives a more detailed understanding of the nature of how price develops, but is focused on periodic returns in continuous time, where the complexity and time consuming nature of attempting to fit results may be unrealistically difficult to do within a short time frame. By exploring more statistical approaches, such as the Intensity model, the empirical nature of the data can be uncovered and it seems worth re-exploring Structural models from this stronger position. Once the data is thoroughly understood using such statistical approaches it is probably easier and more worthwhile to explore the Structural approaches. A concern is that the returns may be time varying (the effect of Triangles probably decreasing with time) and require the modelling of jumps which will be extremely difficult to estimate using a first passage distribution approach.

Fitting the Intensity GEV model across the entire $(h, \tau)$ surface would have been preferable, but due to numerical estimation instability and non-linearities this proved unrealistic. Originally other models had been tried within the base Intensity model using the trial data; for example Gumbel had been a contender which had easily converged across the surface numerically but usually when tested had poor goodness of fit. Only the GEV Intensity model across $h_{\sigma}$ based slices fitted consistently. It may be that there is another Intensity based distribution, either an alternative model or a slight 
improvement, which fixes these issues.

The Gumbel density is given by

$$
f(x)=\frac{1}{\beta} e^{\frac{x-\mu}{\beta}} e^{-e^{\frac{x-\mu}{\beta}}} .
$$

Although GEV is a particularly flexible distribution, its parameters are unintuitive. For comparison the Gumbel density as seen in (8.1) is simpler and uses parameters location $\mu$ which is equivalent to the median and scale $\beta$ which denotes the spread, which provides a more intuitive framework; this is analogous to the normal distribution's use of mean and standard deviation. In comparison the GEV parameters of location, scale and shape are only clearly understood with experience of extreme value distributions and without this are not easily interpreted. The simple way to deal with this issue was to plot the CDFs to clearly communicate the distribution and goodness of fit.

However, even though the GEV Intensity model fits well and can be used to explore the arrival time data usefully using the split of win and loss arrival time, the tools I expected to be useful from survival analysis were only marginally effective. The cumulative deviations based test is too sensitive to be used as a good measure of fit. Yet it proved to be a far better measure than the other tests available. Additionally, though I have used the test as a repeated confirmation to avoid p-value issues, it would be preferable to use effect size statistics which I view as more reliable.

I had considered various testing or goodness of fit alternatives, one of these being to bootstrap the GEV Intensity model to give confidence intervals for the parameters and give some indication of the overlap between Triangles and Naive. This would have also provided an excellent visual confidence interval about the CDF curves. Unfortunately estimation under GEV is too unstable and error prone to do this. However it is quite possible to create new models which do not suffer from these problems, and the following Section 8.1.1 details one possible example. During the study it was decided that to develop more comprehensive models and customised goodness of fit measures would take considerable time, and so these ideas were not pursued further.

\subsubsection{Intensity Cox Sum}

Using the Intensity based method with the Gumbel distribution could also facilitate insertion of covariates where we replace $X$ by vector $\underline{X}$. This proved to be difficult with Intensity based GEV and the extra complexity was not absolutely needed. Originally through the use of vectors was how I attempted to fit the Intensity GEV model across the set of data; however, this was not a simple case of a $\beta^{T} \underline{X}$ linear relationship as it was highly unstable to fit due to the exponential terms. To solve this I normalised by using $h_{\sigma}$ rather than $h$ and fitted across slices of $h$. To create a better fit and to solve some of the issues of fitting across a surface and use covariates, it is possible to use a Cox model 
with a sum of intensities within the base Intensity model, as shown below.

First consider the likelihood function as seen in (5.6) and modify to use a vector of covariates

$$
\begin{aligned}
L\left(t_{k}, t_{k}+d t\right) & =\mathbb{P}\left(t_{k} \leq \tau<t_{k}+d t, \delta \mid F_{t_{k}}\right) \\
& =\mathbb{P}\left(\tau \geq t_{k}, \delta \mid F_{t_{k}}\right) \mathbb{P}\left(\tau<t_{k}+d t, \delta \mid \tau \geq t_{k}, F_{t_{k}}\right) \\
& =e^{-\Lambda\left(t_{k}, \underline{X}\right)}\left[d \Lambda^{(+)}\left(t_{k}, \underline{X}\right)\right]^{\delta_{k}}\left[d \Lambda^{(-)}\left(t_{k}, \underline{X}\right)\right]^{1-\delta_{k}} \\
\prod_{k} L\left(t_{k}, t_{k}+d t\right) & =\prod_{k} e^{-\Lambda\left(t_{k}, \underline{X}\right)}\left[d \Lambda^{(+)}\left(t_{k}, \underline{X}\right)\right]^{\delta_{k}}\left[d \Lambda^{(-)}\left(t_{k}, \underline{X}\right)\right]^{1-\delta_{k}} .
\end{aligned}
$$

Then inject a Cox model based on a sum of intensities into the Likelihood function as

$$
\begin{aligned}
\Lambda^{( \pm)}(t, \underline{X}) & =\int_{0}^{t} e^{\beta^{T} \underline{X}_{u}} d \Lambda_{0}^{( \pm)}(u) \\
\text { where } \Lambda_{0}^{( \pm)}(t) & =\sum_{k} \lambda_{k}^{( \pm)} \mathbb{1}_{\left(t_{k} \leq t\right)} \\
\Longrightarrow \quad \Lambda^{( \pm)}(t, \underline{X}) & =\sum_{k} \lambda_{k}^{( \pm)} e^{\beta^{T} \underline{X}_{k}} \mathbb{1}_{\left(t_{k} \leq t\right)} \\
d \Lambda^{( \pm)}(t, \underline{X}) & \triangleq \Lambda^{( \pm)}(t, \underline{X})-\Lambda^{( \pm)}(t-d t, \underline{X}) \\
& =\sum_{k} \lambda_{k}^{( \pm)} e^{\beta^{T} \underline{X}_{k}}\left(\mathbb{1}_{\left(t_{k} \leq t\right)}-\mathbb{1}_{\left(t_{k} \leq t-d t\right)}\right) \\
& =\sum_{k} \lambda_{k}^{( \pm)} e^{\beta^{T} \underline{X}_{k}} \mathbb{1}_{\left(t-d t<t_{k} \leq t\right)} \\
d \Lambda^{( \pm)}\left(\tau_{i}, \underline{X}\right) & =\sum_{k} \lambda_{k}^{( \pm)} e^{\beta^{T} \underline{X}_{k}} \mathbb{1}_{\left(t_{k} \leq \tau_{i}<t_{k}+d t\right)} .
\end{aligned}
$$

This is defined over a set of $\lambda_{k}$ intensities, where over each $\lambda$ there is a Dirac delta function, but in between the density mass is zero. This is best visualised as seen in Figure 8.1 where it can be seen that the $\Lambda$ contribution consists of the sum of stepwise $\lambda_{k}$ contributions from intensities from each $\tau_{k}$ arrival (this diagram is the same for each + or - intensity sum).

Each $\lambda_{k}$ contribution can be estimated numerically using maximum likelihood. This avoids the need to conform to fit to a distribution which may not map to the underlying data well, ideally fitting fairly exactly to the data and allowing a more specific use for covariates to be mapped. Also this ideally avoids instability issues of nested exponential terms. Additionally arrival times are binomial, so a continuous time approximation is not needed.

Though I have assumed the contributions of covariates to be linear as $\beta \underline{X}$, this may not be the case; and there may be a functional relationship of some nature $g(\beta, \underline{X})$ to account for 


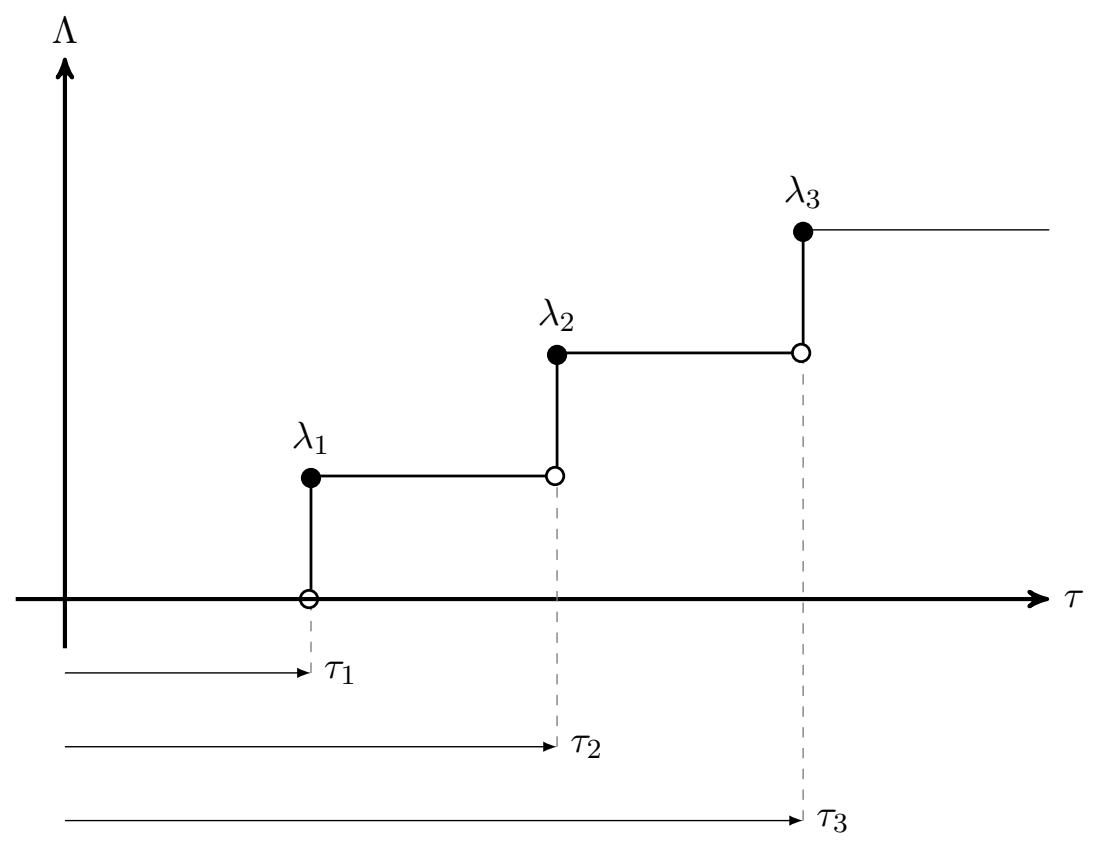

Figure 8.1: Intensity Cox Sum Step Function

non-linearities, as was found with the GEV distribution, but which could not be resolved across the $h$ based surface.

This formulation effectively requires some numerical optimisation rather than standard maximum likelihood derivative methods, and due to additional complexity transparency of understanding is lost; considering this, machine learning should be considered for predictive value and this seems a natural transition from the Cox based model. The current modelling uses full transitions to $\pm h$ without limiting the time constraints, as is done with the effect size heuristics data; it seems more feasible with machine learning to account for partial $h$ values within time constrained bounds.

I suspect with more comprehensive models such as the Intensity Cox Sum it is possible to fit across the entire $h$ dimension of the $(\tau, h)$ surface, then include other covariates. However there are issues to resolve with goodness of fit and with the ability to more intuitively check the results, which dealing with the data in $h$ based slices allows. Some of these methods also add complexity, but I consider that these are best explored after effects have been well established using simpler and more direct methods; although it is certainly possible that an elegant solution can be found, this could take considerable effort to find. 


\subsection{Extensions}

\subsubsection{General Pattern Framework}

The literature ideologically evaluates Technical analysis in a lock step way: done by identifying a technical pattern as seen in text books, calculating periodic returns (without considering how the patterns are traded), and then does some form of null hypothesis p-value test as compared with efficient markets. Every single paper I can find on pattern trading does the same routine with only slight changes. None of the pattern based trading literature I read identified the support and resistance concepts and how this is used to time the market. This study has deliberately gone to some effort to understand how trading is done using such ideas, and from this simple techniques have been developed to statistically evaluate how these patterns are traded. This has been relatively successful as is shown in the transparency of the results.

The temptation in generalising further is to identify more patterns and blindly apply a method as was done by the pattern trading literature, but it is worth again carefully considering the nature of how these patterns are used. I believe the general idea is not about identifying textbook patterns but rather humans trying to make sense of market congestion and attempting to time the market in an attempt to take advantage of forecastability of momentum. This seems the better avenue to explore in more detail, though in a more general way. Towards this, some empirical work should ideally be done interviewing traders; and on the understanding gained, work on methods to capture general congestion and momentum effects. There is however a danger in such an approach of data snooping and spurious results obtained from repeatedly trialling patterns.

Due to pattern detection being rather subjective the method used in this study uses numerous simplifications to capture patterns; for example I deal with results in a simplified manner, and focus on a certain type of Triangle. This is probably the correct approach in order to develop a complete study in a short time frame. Ideally the scope should be widened to both detect other patterns and use a more general selection process, and in doing so unwind many of these simplifications in order to develop a wider understanding. The $\mathcal{R}=1$ approach should be relaxed to take into account asymmetric selectable levels and deal with time limited returns rather than only price level returns. This could then take into account trend continuation. Then instead of using a heuristic like 'half the height', specific times and/or price levels can be used. Also, instead of specific points, as in four relative extrema for Triangles, the number of extrema points should be flexible. In short I would redevelop the entire framework to be more general; however likely in Python rather than R. Using $\mathrm{R}$ code is good for processing statistics and simple prototyping but such a framework would require a more fully fledged language which contains more conventional computational 
abstractions.

This is daunting and may require a new mathematical way to deal with prices, but I can see that this is quite possible. It would be interesting to consider a vector based model which evaluates the effect of relative extrema related to a later price in terms of effect vectors. So instead of viewing pattern strategies in books and robotically trying to implement them, consider each relative extremum as having a directional momentum effect on a later price level. Each vector could then be ascribed weightings. This could fit well within a machine learning context.

\subsubsection{Portfolio Profit and Loss Analysis}

I had considered using direct investment amounts to implement a holding period based return rather than the leveraged \pm 1 return approach, but had rejected this as being too far from how real traders would trade. The caveat is that leveraged returns are not directly comparable to periodic returns or holding period returns, though they could certainly be extracted from the leveraged returns. A better solution is to consider trading as a long term portfolio management scenario; where a portfolio of short term trades is managed and this is compared with a benchmark such as the S\&P 500, using measures such as the Sharp or Sortino ratio. Due to leverage, liquidity and transaction costs this is complex. Additionally US equity markets have regulations, such as variable short sell restrictions which must be adhered to under simulation.

Leverage considerations should take into account systematic market risk and leverage constraints. So the leverage $\mathcal{R}$ based risk model would need to be extended to take into account both a time and positional based risk, which would likely be based on downside volatility portfolio measures similar to the Sortino ratio. Leverage makes use of larger equity positions over shorter periods than buy and hold strategies; and so generally requires greater security liquidity to ensure that trade entries do not adversely affect transaction prices. This is easily done by only simulating trades for which the stock has a liquidity level high enough to ensure that trades do not affect prices. This avoids transaction cost slippage inaccuracies. Consideration of liquidity levels also implies restricted capacity for the trading strategy since trade size will be limited.

Transaction costs can be partly calculated by Bid / Ask spread but also requires taking into account brokerage fees, and I do not have access to accurate historical data for this. This could probably be estimated but would require access to more detailed historical costs.

Finally, using leverage increases risk and this must be accounted for as well. If the market has a sudden move or crashes it is likely stocks will be highly correlated, and exiting positions will also incur large slippage costs. This could result in large losses. Under simulation it would then be sensible to limit the equity or leverage used to realistically avoid excessive losses. Also the equity used for the portfolio of trades should be comparable with a buy and hold strategy to create a reasonable comparison. 
Some fair, comparative way to do this would need to be created which does not mine the data. 


\section{Chapter 9}

\section{Conclusion}

The results indicate that there have historically been market timing advantages of Triangles which show a positive contribution to the returns of US equities as compared with the Naive random sampling case; however, this advantage has been decreasing over time from years 1960 to 2017.

In the cases involving long trades, if the raw profit and loss information is considered then this effect appears significant potentially to 2017; however, when this is adjusted to take into account volatility then this conclusion may be considerably less valid.

Across all cases, using 10 day leveraged constrained return heuristics, and using Cohen's d to standardise over volatility, historically Triangles have had a weak return effect size but all types measured as of time period 2008 to 2017 show a negligible effect. These results are confirmed by the sub-distributions of the Intensity GEV model. As of this last time period there appears to be little or no difference between Triangle and Naive trades when returns are normalised by volatility. It does appear that there has been a predictable historical timing value of Triangles; however it is likely that this trading method has weakened considerably and is now of negligible value. Also, this effect has probably been less valuable than results may indicate, since measurements have not taken into account transaction costs. 


\section{Appendix A}

\section{Terms and Abbreviations}

Table A.1: General Terms and Abbreviations

\begin{tabular}{ll}
\hline Term & Description \\
\hline EMH & Efficient Markets Hypothesis \\
AMH & Adaptive Markets Hypothesis \\
OHLC Prices & Open High Low Close prices \\
CRSP & Center for Research in Security Prices \\
Naive Trade & Trade meant to represent Triangle trades without timing advantage \\
GBPUSD & Pound US Dollar spot currency prices \\
ES & E-mini futures prices \\
CDF & Cumulative Distribution Function \\
ECDF & Empirical Cumulative Distribution Function \\
PDF & Probability Density Function \\
EPDF & Empirical Probability Distribution Function \\
ES & S\&P 500 CME Group Futures \\
OTC & Over the Counter \\
pip & Unit of measure to express the change in value of a spot currency pair \\
\hline
\end{tabular}

Table A.2: Return Terms

\begin{tabular}{ll}
\hline Term & Description \\
\hline Periodic return & Uses standardised periods $r_{i}=\frac{p_{i}-p_{j}}{p_{j}}$, e.g. daily returns \\
Real return & Adjusted using inflation \\
Leveraged return & Defined by how much is gained or lost from the trade \\
Bet return & Excludes time to give amount gained or lost \\
Holding period return & Calculated over a holding period including income \\
Actual return & Produced by a real buy and sell round trip
\end{tabular}


Abnormal returns

Return which is not explained under EMH

Cumulative abnormal return (CAR) Sum of all abnormal returns 


\section{Appendix B}

\section{US Equity Data}

Daily OHLC equity Center for Research in Security Prices (CRSP) data is used from 1960 to 2017 inclusive. This data is adjusted for dividends such that prices reflect proceeds inclusive of dividend gains. Hence with stocks the wins and losses include gains from dividends, admittedly this makes the accuracy of gains slightly inaccurate; however in building the model I ignore this, similarly to many back-tests. All model estimates are done or reported on the basis of discrete wins (+1) and losses (-1), and all Effect Size Heuristics and back-testing are done on the complete dataset including partial wins and losses.

The data is cut into contiguous segments of about 10 years, though with the initial starting segment longer, resulting in forward time intervals starting and ending at the following successive points: 1960-01-01, 1978-01-01, 1988-01-01, 1998-01-01, 2008-01-01 and 2018-01-01. The date division is done at the Triangle break point such that there is some overlap between segments over segment $B C$, in that the actual simulated trade will often fall into the next yearly time segment.

\section{B.1 Exploratory Statistics}

Table B.1: US Equity Triangle Summary Statistics

\begin{tabular}{|c|c|c|c|c|c|c|c|c|}
\hline Type & $h_{\sigma}$ & Period & Count & $r_{b}$ & Mean $\tau$ & Median $\tau$ & Median $\tau^{+}$ & Median $\tau^{-}$ \\
\hline TTop Up & {$\left[\begin{array}{lll}0 & 3\end{array}\right)$} & 1960-01-01 1978-01-01 & 8574 & 0.293 & 15.9 & 8 & 5 & 12 \\
\hline TTop Up & {$\left[\begin{array}{lll}0 & 3\end{array}\right)$} & 1978-01-01 1988-01-01 & 8532 & 0.328 & 16.1 & 8 & 6 & 12 \\
\hline TTop Up & {$\left[\begin{array}{lll}0 & 3\end{array}\right)$} & 1988-01-01 1998-01-01 & 10791 & 0.261 & 16.0 & 7 & 6 & 10 \\
\hline TTop Up & {$\left[\begin{array}{lll}0 & 3\end{array}\right)$} & 1998-01-01 2008-01-01 & 11530 & 0.184 & 15.9 & 7 & 6 & 9 \\
\hline TTop Up & {$\left[\begin{array}{lll}0 & 3\end{array}\right)$} & 2008-01-01 2018-01-01 & 9839 & 0.142 & 16.5 & 8 & 8 & 8 \\
\hline TTop Up & {$[36)$} & 1960-01-01 1978-01-01 & 2682 & 0.221 & 21.4 & 12 & 9 & 16 \\
\hline TTop Up & {$[36)$} & 1978-01-01 1988-01-01 & 2805 & 0.255 & 22.1 & 12 & 10 & 15 \\
\hline TTop Up & {$[36)$} & 1988-01-01 1998-01-01 & 4203 & 0.222 & 21.6 & 12 & 10 & 15 \\
\hline TTop Up & {$[36)$} & 1998-01-01 2008-01-01 & 6785 & 0.133 & 21.9 & 12 & 12 & 12 \\
\hline TTop Up & [3 6) & 2008-01-01 2018-01-01 & 6533 & 0.077 & 21.8 & 12 & 13 & 11 \\
\hline
\end{tabular}


Table B.1: US Equity Triangle Summary Statistics (continued)

\begin{tabular}{|c|c|c|c|c|c|c|c|c|}
\hline Type & $h_{\sigma}$ & Period & Count & $r_{b}$ & Mean $\tau$ & Median $\tau$ & Median $\tau^{+}$ & Median $\tau^{-}$ \\
\hline TTop Up & {$[6 \infty)$} & 1960-01-01 1978-01-01 & 1831 & 0.112 & 30.0 & 18 & 13 & 24 \\
\hline TTop Up & {$[6 \infty)$} & 1978-01-01 1988-01-01 & 1751 & 0.211 & 31.3 & 18 & 15 & 21 \\
\hline TTop Up & {$[6 \infty)$} & 1988-01-01 1998-01-01 & 2829 & 0.174 & 29.5 & 17 & 15 & 19 \\
\hline TTop Up & {$[6 \infty)$} & 1998-01-01 2008-01-01 & 7188 & 0.058 & 30.3 & 17 & 17 & 17 \\
\hline TTop Up & {$[6 \infty)$} & 2008-01-01 2018-01-01 & 9377 & 0.093 & 26.8 & 16 & 17 & 15 \\
\hline TTop Down & [0 3) & 1960-01-01 1978-01-01 & 9414 & 0.117 & 21.8 & 11 & 10 & 13 \\
\hline TTop Down & [0 3) & 1978-01-01 1988-01-01 & 8640 & 0.094 & 21.5 & 11 & 9 & 13 \\
\hline TTop Down & [03) & 1988-01-01 1998-01-01 & 11287 & 0.079 & 21.4 & 10 & 9 & 11 \\
\hline TTop Down & {$\left[\begin{array}{lll}0 & 3\end{array}\right)$} & 1998-01-01 2008-01-01 & 11573 & -0.014 & 22.3 & 10 & 10 & 10 \\
\hline TTop Down & {$\left[\begin{array}{lll}0 & 3\end{array}\right)$} & 2008-01-01 2018-01-01 & 8704 & -0.044 & 21.8 & 9 & 9 & 9 \\
\hline TTop Down & {$[36)$} & 1960-01-01 1978-01-01 & 2658 & 0.113 & 25.7 & 14 & 13 & 16 \\
\hline TTop Down & {$[36)$} & 1978-01-01 1988-01-01 & 2692 & 0.079 & 27.4 & 15 & 13 & 18 \\
\hline TTop Down & {$[36)$} & 1988-01-01 1998-01-01 & 3652 & 0.042 & 26.1 & 13 & 12 & 15 \\
\hline TTop Down & {$[36)$} & 1998-01-01 2008-01-01 & 5838 & -0.010 & 24.9 & 13 & 12 & 14 \\
\hline TTop Down & {$[36)$} & 2008-01-01 2018-01-01 & 5011 & -0.024 & 23.8 & 12 & 11 & 12 \\
\hline TTop Down & {$[6 \infty)$} & 1960-01-01 1978-01-01 & 1786 & 0.169 & 37.1 & 19 & 18 & 20 \\
\hline TTop Down & {$[6 \infty)$} & 1978-01-01 1988-01-01 & 1654 & 0.134 & 33.3 & 19 & 16 & 22 \\
\hline TTop Down & {$[6 \infty)$} & 1988-01-01 1998-01-01 & 2495 & 0.046 & 36.7 & 18 & 17 & 20 \\
\hline TTop Down & {$[6 \infty)$} & 1998-01-01 2008-01-01 & 5872 & 0.041 & 34.6 & 17 & 16 & 18 \\
\hline TTop Down & {$[6 \infty)$} & 2008-01-01 2018-01-01 & 7074 & -0.041 & 28.4 & 14 & 13 & 15 \\
\hline TBot Up & {$\left[\begin{array}{lll}0 & 3\end{array}\right)$} & 1960-01-01 1978-01-01 & 8734 & 0.236 & 16.7 & 8 & 6 & 12 \\
\hline TBot Up & [0 3) & 1978-01-01 1988-01-01 & 8028 & 0.317 & 17.1 & 8 & 6 & 13 \\
\hline TBot Up & [0 3) & 1988-01-01 1998-01-01 & 11733 & 0.238 & 16.8 & 8 & 6 & 11 \\
\hline TBot Up & {$[03)$} & 1998-01-01 2008-01-01 & 12080 & 0.149 & 17.7 & 8 & 7 & 10 \\
\hline TBot Up & {$\left[\begin{array}{lll}0 & 3\end{array}\right)$} & 2008-01-01 2018-01-01 & 9500 & 0.091 & 18.2 & 9 & 8 & 10 \\
\hline TBot Up & {$[36)$} & 1960-01-01 1978-01-01 & 2218 & 0.148 & 22.2 & 12 & 10 & 16 \\
\hline TBot Up & {$[36)$} & 1978-01-01 1988-01-01 & 2188 & 0.236 & 23.0 & 13 & 11 & 18 \\
\hline TBot Up & {$[36)$} & 1988-01-01 1998-01-01 & 3145 & 0.211 & 23.3 & 13 & 11 & 16 \\
\hline TBot Up & {$[36)$} & 1998-01-01 2008-01-01 & 5404 & 0.120 & 23.4 & 13 & 12 & 15 \\
\hline TBot Up & {$[36)$} & 2008-01-01 2018-01-01 & 4893 & 0.049 & 24.2 & 13 & 14 & 13 \\
\hline TBot Up & {$[6 \infty)$} & 1960-01-01 1978-01-01 & 1437 & 0.122 & 28.7 & 16 & 13 & 20 \\
\hline TBot Up & {$[6 \infty)$} & 1978-01-01 1988-01-01 & 1216 & 0.183 & 27.0 & 16 & 14 & 21 \\
\hline TBot Up & {$[6 \infty)$} & 1988-01-01 1998-01-01 & 1834 & 0.167 & 28.3 & 16 & 14 & 19 \\
\hline TBot Up & {$[6 \infty)$} & 1998-01-01 2008-01-01 & 4739 & 0.034 & 29.9 & 17 & 15 & 19 \\
\hline TBot Up & {$[6 \infty)$} & 2008-01-01 2018-01-01 & 6123 & 0.052 & 31.3 & 16 & 17 & 14 \\
\hline TBot Down & {$\left[\begin{array}{lll}0 & 3\end{array}\right)$} & 1960-01-01 1978-01-01 & 12016 & 0.141 & 21.8 & 11 & 11 & 12 \\
\hline TBot Down & {$\left[\begin{array}{lll}0 & 3\end{array}\right)$} & 1978-01-01 1988-01-01 & 10170 & 0.075 & 22.3 & 11 & 10 & 12 \\
\hline TBot Down & {$\left[\begin{array}{lll}0 & 3\end{array}\right)$} & 1988-01-01 1998-01-01 & 13988 & 0.075 & 23.4 & 10 & 10 & 11 \\
\hline TBot Down & [0 3) & 1998-01-01 2008-01-01 & 14810 & 0.047 & 24.7 & 11 & 11 & 11 \\
\hline TBot Down & {$\left[\begin{array}{lll}0 & 3\end{array}\right)$} & 2008-01-01 2018-01-01 & 10699 & -0.030 & 21.4 & 10 & 10 & 11 \\
\hline TBot Down & {$[36)$} & 1960-01-01 1978-01-01 & 3352 & 0.153 & 25.2 & 15 & 13 & 17 \\
\hline TBot Down & {$[36)$} & 1978-01-01 1988-01-01 & 2872 & 0.095 & 27.4 & 16 & 14 & 17 \\
\hline TBot Down & {$[36)$} & 1988-01-01 1998-01-01 & 3579 & 0.055 & 25.6 & 14 & 13 & 15 \\
\hline TBot Down & {$[36)$} & 1998-01-01 2008-01-01 & 6279 & 0.038 & 27.1 & 13 & 13 & 14 \\
\hline TBot Down & {$[36)$} & 2008-01-01 2018-01-01 & 5498 & -0.023 & 24.2 & 13 & 11 & 14 \\
\hline TBot Down & {$[6 \infty)$} & 1960-01-01 1978-01-01 & 2183 & 0.179 & 30.0 & 17 & 16 & 20 \\
\hline TBot Down & {$[6 \infty)$} & 1978-01-01 1988-01-01 & 1732 & 0.143 & 32.6 & 18 & 17 & 20 \\
\hline TBot Down & {$[6 \infty)$} & 1988-01-01 1998-01-01 & 2357 & 0.047 & 34.2 & 18 & 18 & 18 \\
\hline TBot Down & {$[6 \infty)$} & 1998-01-01 2008-01-01 & 5932 & 0.062 & 35.0 & 18 & 17 & 18 \\
\hline
\end{tabular}


Table B.1: US Equity Triangle Summary Statistics (continued)

\begin{tabular}{lllrrrrrr}
\hline Type & $h_{\sigma}$ & Period & Count & $r_{b}$ & Mean $\tau$ & Median $\tau$ & Median $\tau^{+}$ & Median $\tau^{-}$ \\
\hline TBot Down & {$[6 \infty)$} & $2008-01-012018-01-01$ & 6760 & -0.022 & 27.6 & 14 & 12 & 15 \\
\hline
\end{tabular}

Table B.2: US Equity Naive Summary Statistics

\begin{tabular}{|c|c|c|c|c|c|c|c|c|}
\hline Type & $h_{\sigma}$ & Period & Count & $r_{b}$ & Mean $\tau$ & Median $\tau$ & Median $\tau^{+}$ & Median $\tau^{-}$ \\
\hline TTop Up & {$\left[\begin{array}{ll}0 & 3\end{array}\right)$} & 1960-01-01 1978-01-01 & 85740 & 0.069 & 33.2 & 8 & 10 & 7 \\
\hline TTop Up & [03) & 1978-01-01 1988-01-01 & 85320 & 0.119 & 29.6 & 8 & 10 & 7 \\
\hline TTop Up & {$[03)$} & 1988-01-01 1998-01-01 & 107910 & 0.112 & 30.0 & 8 & 9 & 6 \\
\hline TTop Up & [0 3) & 1998-01-01 2008-01-01 & 115300 & 0.060 & 24.8 & 6 & 7 & 5 \\
\hline TTop Up & {$[03)$} & 2008-01-01 2018-01-01 & 98390 & 0.071 & 19.3 & 6 & 7 & 5 \\
\hline TTop Up & {$[36)$} & 1960-01-01 1978-01-01 & 26820 & 0.119 & 52.5 & 16 & 18 & 14 \\
\hline TTop Up & {$[36)$} & 1978-01-01 1988-01-01 & 28050 & 0.188 & 49.3 & 17 & 20 & 14 \\
\hline TTop Up & {$[36)$} & 1988-01-01 1998-01-01 & 42030 & 0.202 & 46.7 & 16 & 18 & 13 \\
\hline TTop Up & {$[36)$} & 1998-01-01 2008-01-01 & 67850 & 0.109 & 36.6 & 12 & 14 & 11 \\
\hline TTop Up & {$[36)$} & 2008-01-01 2018-01-01 & 65330 & 0.096 & 28.1 & 11 & 12 & 9 \\
\hline TTop Up & {$[6 \infty)$} & 1960-01-01 1978-01-01 & 18310 & 0.175 & 89.4 & 29 & 35 & 24 \\
\hline TTop Up & {$[6 \infty)$} & 1978-01-01 1988-01-01 & 17510 & 0.264 & 91.3 & 32 & 37 & 24 \\
\hline TTop Up & {$[6 \infty)$} & 1988-01-01 1998-01-01 & 28290 & 0.254 & 73.1 & 26 & 31 & 20 \\
\hline TTop Up & {$[6 \infty)$} & 1998-01-01 2008-01-01 & 71880 & 0.163 & 69.6 & 23 & 27 & 19 \\
\hline TTop Up & {$[6 \infty)$} & 2008-01-01 2018-01-01 & 93770 & 0.132 & 45.2 & 18 & 21 & 15 \\
\hline TTop Down & {$[03)$} & 1960-01-01 1978-01-01 & 94140 & -0.060 & 34.9 & 9 & 8 & 10 \\
\hline TTop Down & {$\left[\begin{array}{ll}0 & 3\end{array}\right)$} & 1978-01-01 1988-01-01 & 86400 & -0.094 & 32.7 & 9 & 8 & 10 \\
\hline TTop Down & [0 3) & 1988-01-01 1998-01-01 & 112870 & -0.084 & 33.6 & 8 & 7 & 10 \\
\hline TTop Down & [0 3) & 1998-01-01 2008-01-01 & 115730 & -0.029 & 27.0 & 7 & 6 & 7 \\
\hline TTop Down & {$\left[\begin{array}{lll}0 & 3\end{array}\right)$} & 2008-01-01 2018-01-01 & 87040 & -0.058 & 22.9 & 7 & 6 & 8 \\
\hline TTop Down & {$[36)$} & 1960-01-01 1978-01-01 & 26580 & -0.116 & 57.0 & 17 & 14 & 19 \\
\hline TTop Down & {$[36)$} & 1978-01-01 1988-01-01 & 26920 & -0.189 & 52.5 & 19 & 15 & 22 \\
\hline TTop Down & {$[36)$} & 1988-01-01 1998-01-01 & 36520 & -0.172 & 48.3 & 16 & 14 & 19 \\
\hline TTop Down & {$[36)$} & 1998-01-01 2008-01-01 & 58380 & -0.090 & 39.7 & 13 & 11 & 15 \\
\hline TTop Down & {$[36)$} & 2008-01-01 2018-01-01 & 50110 & -0.092 & 31.8 & 12 & 10 & 14 \\
\hline TTop Down & {$[6 \infty)$} & 1960-01-01 1978-01-01 & 17860 & -0.165 & 96.6 & 31 & 24 & 37 \\
\hline TTop Down & {$[6 \infty)$} & 1978-01-01 1988-01-01 & 16540 & -0.259 & 89.3 & 32 & 24 & 39 \\
\hline TTop Down & {$[6 \infty)$} & 1988-01-01 1998-01-01 & 24950 & -0.218 & 81.2 & 27 & 21 & 33 \\
\hline TTop Down & {$[6 \infty)$} & 1998-01-01 2008-01-01 & 58720 & -0.130 & 78.0 & 24 & 20 & 28 \\
\hline TTop Down & {$[6 \infty)$} & 2008-01-01 2018-01-01 & 70740 & -0.129 & 50.2 & 19 & 16 & 22 \\
\hline TBot Up & {$\left[\begin{array}{lll}0 & 3\end{array}\right)$} & 1960-01-01 1978-01-01 & 87340 & 0.052 & 30.6 & 8 & 8 & 7 \\
\hline TBot Up & {$[03)$} & 1978-01-01 1988-01-01 & 80280 & 0.076 & 26.6 & 7 & 8 & 6 \\
\hline TBot Up & {$\left[\begin{array}{ll}0 & 3\end{array}\right)$} & 1988-01-01 1998-01-01 & 117330 & 0.061 & 26.9 & 7 & 8 & 6 \\
\hline TBot Up & [0 3) & 1998-01-01 2008-01-01 & 120800 & 0.025 & 22.8 & 6 & 7 & 5 \\
\hline TBot Up & {$\left[\begin{array}{lll}0 & 3\end{array}\right)$} & 2008-01-01 2018-01-01 & 95000 & 0.046 & 20.5 & 6 & 7 & 5 \\
\hline TBot Up & {$[36)$} & 1960-01-01 1978-01-01 & 22180 & 0.106 & 48.9 & 16 & 18 & 14 \\
\hline TBot Up & {$[36)$} & 1978-01-01 1988-01-01 & 21880 & 0.170 & 48.2 & 17 & 19 & 14 \\
\hline TBot Up & {$[36)$} & 1988-01-01 1998-01-01 & 31450 & 0.146 & 43.9 & 15 & 17 & 13 \\
\hline TBot Up & {$[36)$} & 1998-01-01 2008-01-01 & 54040 & 0.077 & 35.5 & 13 & 14 & 12 \\
\hline TBot Up & {$[36)$} & 2008-01-01 2018-01-01 & 48930 & 0.085 & 30.9 & 12 & 14 & 10 \\
\hline TBot Up & {$[6 \infty)$} & 1960-01-01 1978-01-01 & 14370 & 0.141 & 79.3 & 28 & 33 & 23 \\
\hline TBot Up & {$[6 \infty)$} & 1978-01-01 1988-01-01 & 12160 & 0.205 & 74.8 & 27 & 32 & 21 \\
\hline
\end{tabular}


Table B.2: US Equity Naive Summary Statistics (continued)

\begin{tabular}{|c|c|c|c|c|c|c|c|c|}
\hline Type & $h_{\sigma}$ & Period & Count & $r_{b}$ & Mean $\tau$ & Median $\tau$ & Median $\tau^{+}$ & Median $\tau^{-}$ \\
\hline TBot Up & {$[6 \infty)$} & 1988-01-01 1998-01-01 & 18340 & 0.163 & 61.5 & 23 & 27 & 19 \\
\hline TBot Up & {$[6 \infty)$} & 1998-01-01 2008-01-01 & 47390 & 0.090 & 66.5 & 23 & 26 & 20 \\
\hline TBot Up & {$[6 \infty)$} & 2008-01-01 2018-01-01 & 61230 & 0.121 & 52.9 & 21 & 24 & 18 \\
\hline TBot Down & [03) & 1960-01-01 1978-01-01 & 120160 & -0.050 & 34.7 & 8 & 8 & 9 \\
\hline TBot Down & {$\left[\begin{array}{ll}0 & 3\end{array}\right)$} & 1978-01-01 1988-01-01 & 101700 & -0.061 & 31.4 & 9 & 8 & 10 \\
\hline TBot Down & [03) & 1988-01-01 1998-01-01 & 139880 & -0.040 & 31.3 & 8 & 7 & 9 \\
\hline TBot Down & {$[03)$} & 1998-01-01 2008-01-01 & 148100 & 0.004 & 26.3 & 6 & 6 & 7 \\
\hline TBot Down & {$[03)$} & 2008-01-01 2018-01-01 & 106990 & -0.034 & 22.8 & 7 & 6 & 8 \\
\hline TBot Down & {$[36)$} & 1960-01-01 1978-01-01 & 33520 & -0.106 & 57.1 & 17 & 15 & 20 \\
\hline TBot Down & {$[36)$} & 1978-01-01 1988-01-01 & 28720 & -0.146 & 52.1 & 18 & 15 & 20 \\
\hline TBot Down & {$[36)$} & 1988-01-01 1998-01-01 & 35790 & -0.126 & 46.4 & 16 & 14 & 18 \\
\hline TBot Down & {$[36)$} & 1998-01-01 2008-01-01 & 62790 & -0.064 & 39.9 & 14 & 12 & 15 \\
\hline TBot Down & {$[36)$} & 2008-01-01 2018-01-01 & 54980 & -0.071 & 34.1 & 13 & 11 & 15 \\
\hline TBot Down & {$[6 \infty)$} & 1960-01-01 1978-01-01 & 21830 & -0.125 & 90.3 & 29 & 24 & 35 \\
\hline TBot Down & {$[6 \infty)$} & 1978-01-01 1988-01-01 & 17320 & -0.201 & 78.4 & 28 & 22 & 33 \\
\hline TBot Down & {$[6 \infty)$} & 1988-01-01 1998-01-01 & 23570 & -0.140 & 67.0 & 23 & 19 & 27 \\
\hline TBot Down & {$[6 \infty)$} & 1998-01-01 2008-01-01 & 59320 & -0.068 & 75.9 & 25 & 22 & 28 \\
\hline TBot Down & {$[6 \infty)$} & 2008-01-01 2018-01-01 & 67600 & -0.098 & 56.5 & 22 & 18 & 25 \\
\hline
\end{tabular}




\section{Appendix C}

\section{GBPUSD Trial}

GBPUSD is used to trial and develop models seen in Chapters 4 and 5. The issue is that the topic is virtually unexplored and requires a grounded evaluation of which techniques are the most applicable to transparently evaluate the data, this requires an iterative approach and it seems better to report the methods in this context of the use of trial data. This is partly motivated by seeing the lack in clarity of related research and realising the need for alternative approaches to improve this. The point of using trial data is to develop models against realistic results without gaining insight into the US Equity data which could bias the analysis (though from having read the literature, some degree of tacit knowledge is unavoidable).

So the results of this trial using GBPUSD data are not intended to be definitive or complete but rather show the development of ideas which required some data driven insight. This trial then bridges the development of ideas of how best to interpret short term momentum towards the analysis of US Equity based results. In this trial I only report a minimal subset of exploratory data and the ideas which are most useful for explaining the model design. Only surfaces for TTop Up are plotted to save space, since visually the results for each Triangle type are quite similar. Some of these results a repeated in the main thesis for ease of reference.

The end result has been that the Structural based model was not easily applicable, so the Intensity model was instead developed and selected with an adjusted approach to fit US equities. Currency minute period data is sufficiently different to US equity data while having enough similarities to facilitate this. Aside from spot GBPUSD data E-mini futures are explored briefly to check that the ideas map well to time series data more similar to stocks having aspects such as drift, these fairly limited results are reported in Appendix D. The ES results mainly form an alternative baseline to check against the more comprehensive GBPUSD results, and I mention these results only occasionally within the thesis due to this.

Spot currency is reputedly traded using momentum strategies such as Triangles, 
GBPUSD is chosen as a test case being one of several available candidates due to high liquidity and low transaction costs. Here both price changes without transaction costs are examined to establish if Triangles are informative of future price changes, and price changes with transaction costs are examined to establish potential for profitability. Once these raw results are established I investigate momentum further, comparing and contrasting modelling approaches towards a more well developed analytical approach.

\section{C.1 Spot Price Data}

Minute period GBPUSD OHLC spot currency time series data are used from Dukascopy bank from midnight 2003-08-04 to midnight 2017-01-12. The Bid price is used unless otherwise stated. Transaction costs are assumed to be the Bid/Ask spread as we assume the simulated trader trades the interbank rates at high liquidity, so I assume slippage averages to zero and there are no brokerage costs. This is done as we assume traders have a seat on an interbank trading desk with direct OTC access without the need of an intermediary broker. The assumption then is that there are no charges for trading and slippage does not favour either party or involve an intermediary offsetting risk with re-quoting or by slipping prices.

Though I assume returns are leveraged bets, I do not cap leverage or account for cost of carry under the paradigm of analysing for potential information for profit over the 'short term' as opposed to determining longer term out-of-sample profitability (forward testing) where I would take these into account. Short term in this case being a period in which a trader would select to maximize profitability and minimize systematic risk from other factors, this term then is dependent on the Section of probability which would maximize this trade-off from whichever model is chosen to model this. This is one aspect exploration of models are data helps to clarify since the term of trade carry requires some thought to select.

When considering the profitability of transactions before the potential trade I do an initial calculation of the proportional cost due to spread, done to determine if the trade would be viable. If the expected cost of the trade is greater than the expected profit it does not represent a realistic bet that a trader would take, and so this trade is excluded. To simplify this all trades where the transaction cost is greater than $1 / 20$ of the total expected price movement are removed, realistically this is coarse but only a simple heuristic is needed to explore the data and consequences of translation costs is required. For GBPUSD we assume that the average spread is about 1 pip from Dukascopy data (though this is a slightly high estimate taken from rounding up Dukascopy broker bid/ask mean spread) to give a minimum triangle $h$ heuristic of 0.002 .

I represent summary statistics for each case to highlight the effect of transaction costs. The total counts of triangles found in these unrestricted and transaction cost restricted 
cases are given by Table C. 1 with Monte Carlo counts following which are 10 times the size. 10 times though a small increase gives an arguably large sample size while both allowing practical estimation of models through optimisation and providing an ability to practically evaluate the context of individual data points, where a sample size might not.

\section{C.2 Exploratory Statistics}

When considered from the perspective of trade measurements $(h, \tau)$ the stopping time distribution to the close point of trades result in data that in general forms a large head on the left and a long right tail. Understanding this data requires some general exploration of Bet returns, stopping times in terms of $(h, \tau)$ and a rough idea of the distribution of such events which is initially best explored visually until a model is developed. Visual representations are shown in Section C.3.1 with various 3D surfaces. Only a few exploratory measures are used to show some fundamental aspects; later modelling estimations and visualisation adds where appropriate to build on this information - the measurement types are deliberately sparse so as not to confuse the issue.

Table C.1: GBPUSD Triangle Counts

\begin{tabular}{lrrrr}
\hline & TTop Up & TBot Down & TTop Down & TBot Up \\
\hline Count & 10609 & 7944 & 10252 & 10088 \\
Count, $h>0.002$ & 1399 & 1005 & 1410 & 1160 \\
\hline
\end{tabular}

Table C.2: GBPUSD Naive Event Counts

\begin{tabular}{lrrrr}
\hline & TTop Up & TBot Down & TTop Down & TBot Up \\
\hline Count & 106090 & 79440 & 102520 & 100880 \\
Count, $h>0.002$ & 13990 & 10050 & 14100 & 11600 \\
\hline
\end{tabular}

The counts of various Triangle cases are seen in Table C.1, the simple observation being that there is a reasonably large sample size, but when considered in terms of the trades worth taking at the $h>0.002$ level there are only a small number of order 30 viable trades per year for each Triangle type. Considered in context with the Bet Return profitability potential as seen in Table C.5, there does seem to be some small anomaly amount of information present when using the t-test, but when considered after transaction costs only TBot Up appears to show reasonable potential for profit and only minimally so. Sample size and the potential for profit need to be high enough such that realistic results can be evaluated; this is not the case and similarly with ES futures as seen in Appendix D. Effectively transaction costs appear to quickly eliminate most or all of the anomaly 
effect. There is however enough of an informational value in terms of price movement to make an exploration of models worthwhile.

The other obvious difference between the Triangle and Naive cases is in terms of stopping times, this is seen here rather coarsely as the difference in median stopping times between Triangles and the Naive cases, where mostly Triangles show shorter stopping times than their Naive cases, though TTop Up is much the same (ES futures show larger differences).

Table C.3: GBPUSD Triangle Stopping Times

\begin{tabular}{lrrrr}
\hline & TTop Up & TTop Down & TBot Down & TBot Up \\
\hline Mean & 88.1 & 78.7 & 81.3 & 80.2 \\
Median ST & 19.0 & 18.0 & 18.0 & 18.0 \\
Median ST Win & 20.0 & 16.0 & 16.0 & 15.0 \\
Median ST Loss & 19.0 & 20.0 & 20.0 & 21.0 \\
\hline
\end{tabular}

Table C.4: GBPUSD Naive Event Stopping Times

\begin{tabular}{lrrrr}
\hline & TTop Up & TTop Down & TBot Down & TBot Up \\
\hline Mean & 88.1 & 85.4 & 83.8 & 92.3 \\
Median ST & 19.0 & 19.0 & 18.0 & 20.0 \\
Median ST Win & 20.0 & 18.0 & 18.0 & 20.0 \\
Median ST Loss & 19.0 & 19.0 & 18.0 & 20.0 \\
\hline
\end{tabular}

For testing GBPUSD Bet returns I assume the sample mean return is normally distributed about the population mean under the Central Limit Theorem. Under the assumption of zero drift the Null hypothesis is that the mean return of any trade is zero, and since in this case the market is assumed to be efficient any predictive bias from acquired information is both zero and result of random walk. Under this scenario the following return cases are tested using t-tests in Table C.7. As stated this is not intended to be rigorous but rather indicative. In this case p-value based testing is not particularly useful for exploring trading returns due to reasons alluded to in Chapter 2, but this idea seemed worth exploring under EMH reasoning. I later dropped using this type of direct p-value based return testing as not providing enough value to be of use; however the concept was worth exploring and in this simple case is useful.

For the sample where $h>0.002$, returns are explored for information content as 'Raw Bet Returns' and profit potential tested as 'Real Bet Returns'(raw bet returns minus transaction costs). This is to show potential information content verses potential profits, which is strongly affected by transaction costs (also seen with ES futures). 
Table C.5: GBPUSD Triangle Mean Returns

\begin{tabular}{lrrrr}
\hline & TTop Up & TTop Down & TBot Down & TBot Up \\
\hline Raw Bet Return & 0.0217 & 0.0617 & 0.0234 & 0.0449 \\
Raw Bet Return, $h>0.002$ & 0.0207 & 0.0896 & -0.0277 & 0.0588 \\
Real Bet Return, $h>0.002$ & -0.0160 & 0.0524 & -0.0642 & 0.0217 \\
\hline
\end{tabular}

Table C.6: GBPUSD Naive Event Mean Returns

\begin{tabular}{lrrrr}
\hline & TTop Up & TTop Down & TBot Down & TBot Up \\
\hline Raw Bet Return & -0.000251 & 0.000315 & -0.000684 & 0.0000533 \\
Raw Bet Return, $h>0.002$ & 0.001990 & -0.003390 & 0.000632 & -0.0011500 \\
\hline
\end{tabular}

Table C.7: GBPUSD Mean Return P-Values

\begin{tabular}{lrrrr}
\hline & TTop Up & TTop Down & TBot Down & TBot Up \\
\hline Raw Bet Return $=0$ & 0.022 & 0.000 & 0.015 & 0.000 \\
Raw Bet Return $>0, h>0.002$ & 0.011 & 0.000 & 0.007 & 0.000 \\
Real Bet Return $>0, h>0.002$ & 0.750 & 0.032 & 0.997 & 0.204 \\
\hline
\end{tabular}

\section{C.3 Structural Approach}

Table C.8 shows the Structural model parameter estimates for Brownian Motion first passage times. TTop Up and TBot Up types use the first passage above price level; TBot Down and TTop Down use the first passage times bellow in accordance with the direction that the simulated traders expect. Immediately apparent is that estimates of drift appear to be zero but this is more-so that these show small values around 6 decimal places. Having carefully checked both the mathematics and code this seems correct; visually checking the manifold clearly shows the numerical estimation is probably correct but that the gradient of the $\mu$ estimates is so shallow that it requires a significantly small numerical step size to be sure that the $\mu$ estimate is accurate. In contrast to $\mu, \sigma$ estimates show volatility whose magnitude is indicative of the profitability magnitude seen in Section C.2. For example TTop Down shows the highest $\sigma$ estimate and also appears to be the most likely to be profitable, other estimates follow suit. Overall this is may be due to the negative exponential term of the distribution equation biasing the results and seems to imply that the model does not represent the data well. This is no surprise, the raw Brownian Motion model is an initial starting point and is a very process unlikely to magically fit without some modification.

Comparison of Triangle parameter estimates with corresponding Naive cases show differences as seen in tables C.8 and C.9. At the very least, the estimates for Naive cases are similar to what would be expected, since as a baseline the Euler sigma estimate is 0.173 which is very close to the Naive estimates for each type. In summary the $\sigma$ Naive 
cases are reasonably close the Euler estimates and far away from their corresponding Triangle estimates to show some potential, but the model may not fit the data since I would perhaps expect the Triangle estimates to show more drift due to the exploratory data. A question then is how to test these estimates for size of effect or/and significance or how to add effect bounds so as to define how realistic the estimate differences are, how to do this is not clear especially since this requires 'shaky' assumptions about the structure of the model. However before discussing this I explore the results visually to further understand the idea, since this exposes features of the data which are not obvious.

Table C.8: GBPUSD Triangle Brownian Motion First Passage Estimates

\begin{tabular}{rrrrr}
\hline & TTop Up & TTop Down & TBot Down & TBot Up \\
\hline$\sigma$ & 0.206 & 0.327 & 0.211 & 0.261 \\
$\mu$ & 0.000 & 0.000 & 0.000 & 0.000 \\
\hline
\end{tabular}

Table C.9: GBPUSD Naive Brownian Motion First Passage Estimates

\begin{tabular}{rrrrr}
\hline & TTop Up & TTop Down & TBot Down & TBot Up \\
\hline$\sigma$ & 0.167 & 0.158 & 0.178 & 0.167 \\
$\mu$ & 0.000 & 0.000 & 0.000 & 0.000 \\
\hline
\end{tabular}

\section{C.3.1 Surface Exploration}

As an initial exploration I plot a 2 dimensional joint distribution of Triangle heights verses first passage times as seen in Figure C.3, the intuition being that this shows a surface representation of price movements in terms of joint arrival time and $h$ density which can help understand momentum based returns by exploring the change in price to a fixed proportionate level (again this is not a true distribution only an approximation). Overall for comparison I do this for 3 cases: Triangles, Naive sampling (where the sampling is redone to take into account new bounds and only one first passage level as opposed to duel) and first passage times given by theory from the derived Brownian Motion first passage distribution.

Due to the surface shape having particularly long tails and to gain useful insights I restrict the results to stopping times within the range of 1-60 minutes inclusive, and heights from 0 to 0.0025 , then scale the results to have a total combined mass of 1 such that results are comparable (note: this is an approximate comparison). Without these adjustments it is impractical to see the results as most of the mass visually appears in a tiny spike in the corner of the plot - the distributions have a very long left tail which is of less interest, since the concern is short term momentum. 


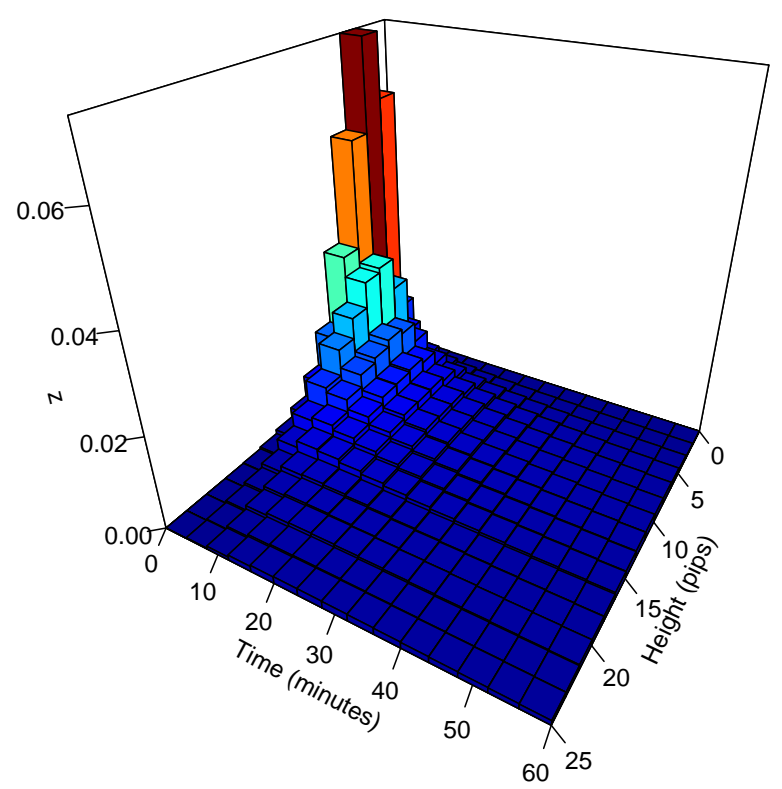

Figure C.1: TBot Up Constrained Brownian Motion 2D First Passage Distribution $\sigma=$ 0.173

Sample heights are generated by taking all triangle heights within this region and generating Naive stopping times which occur in 60 minutes or less. For each height the time series has a point selected and from that point forward it is determined how long it takes for the change in price to go to or above that height price. This is done 10 times for each triangle height to produce a distribution of stopping times that represents Naive stopping times not conditional on Triangles occurring. This is a new sample of Naive heights conditional on first passage in one direction and stopping in 60 minutes or less. This representation is artificial and deliberately data-snoops which a knowledge of the future, which is clearly a bad method but is a useful constrained comparative tool.

Stopping times given by theory are calculated by calculating theoretical densities for each histogram height by time/cell, using the empirical heights from the triangles as a basis. Each height division is given a density contribution weight equal to 1 divided by the total number of heights. The probability of that height stopping within a particular cell is then calculated according to the conditional probability of the first passage time. Each cell's cumulative density is then the sum of each heights cumulative density contribution from times the probability of being in that cell. The mathematics for this is explained in Section 4.2 .

Various plots are shown for TBot Up triangles, other types are not shown as they display 


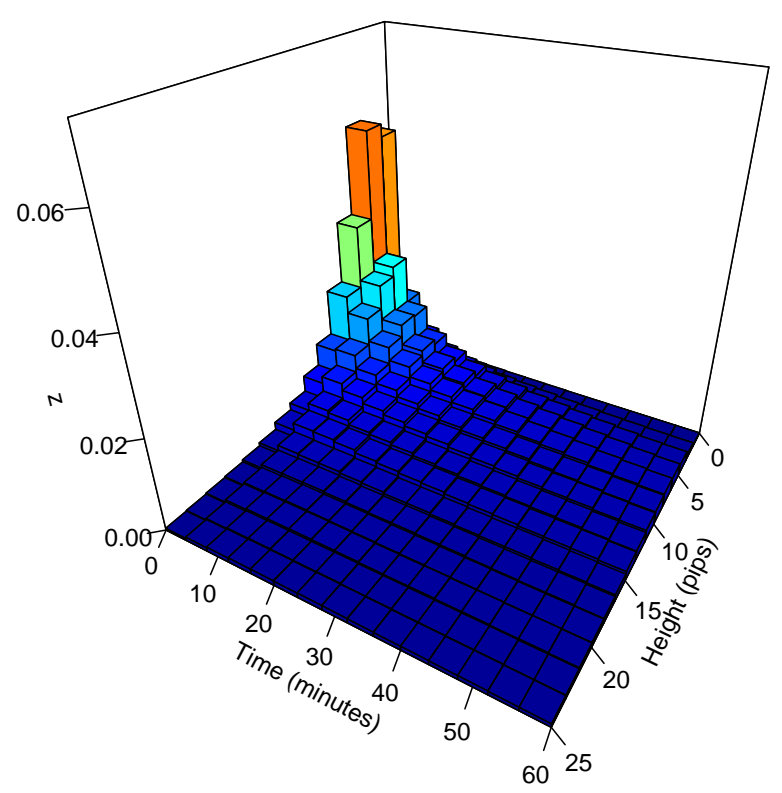

Figure C.2: TBot Up Constrained Naive 2D First Passage Distribution

the same characteristics, though to a greater or lesser degree. I use the TBot Up as an example as it displays something of a middle ground between the different type, enough to display the characteristics without favourably picking the more extreme cases. Figure 4.1 shows the stopping time distribution for one height to gain an insight into how the curve fits within the 2D distribution. Figure C.1 then shows the 2D height vs stopping time distribution as a histogram with comparable sections to the Naive Figure C.2 and Triangle Figure C.3 cases. These are difficult to compare so the Naive verses Brownian Motion in Figure C.5 and Naive verses Triangles in Figure C.4 are compared on the same plot using a kernel smoothed version (Brownian Motion verses Triangles are not shown as this is similar to the Naive verses Triangles case).

From the visualisation there are two main observations that can be seen which show how the Brownian Motion fit is not ideal. Firstly the curve fit comparison changes across the height dimension such that it is not comparable with either the Naive or Triangle cases consistently across $h$, though the Naive case does come much closer to the Brownian Motion fit as is expected. Secondly for higher $h$ values and early stopping times it appears not possible to fit a curve which accounts for distribution changes, this is the region that is most interesting for momentum since it denotes the area where profitable trades would potentially take place such that transaction costs are overcome. Curiously, no matter what the Brownian Motion parameters are it is not possible to get a fit inside the section 


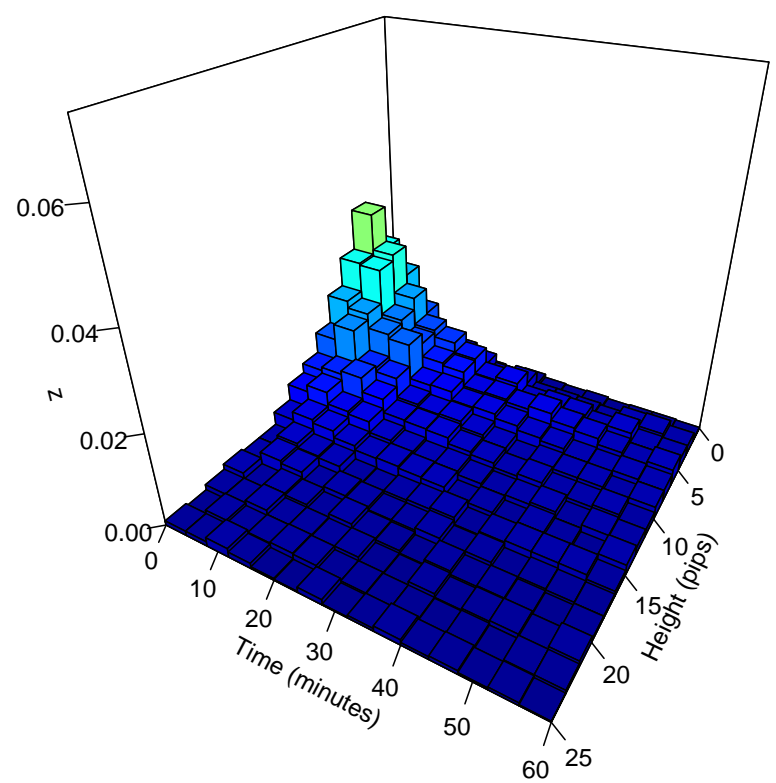

Figure C.3: TBot Up Constrained Triangle 2D First Passage Distribution

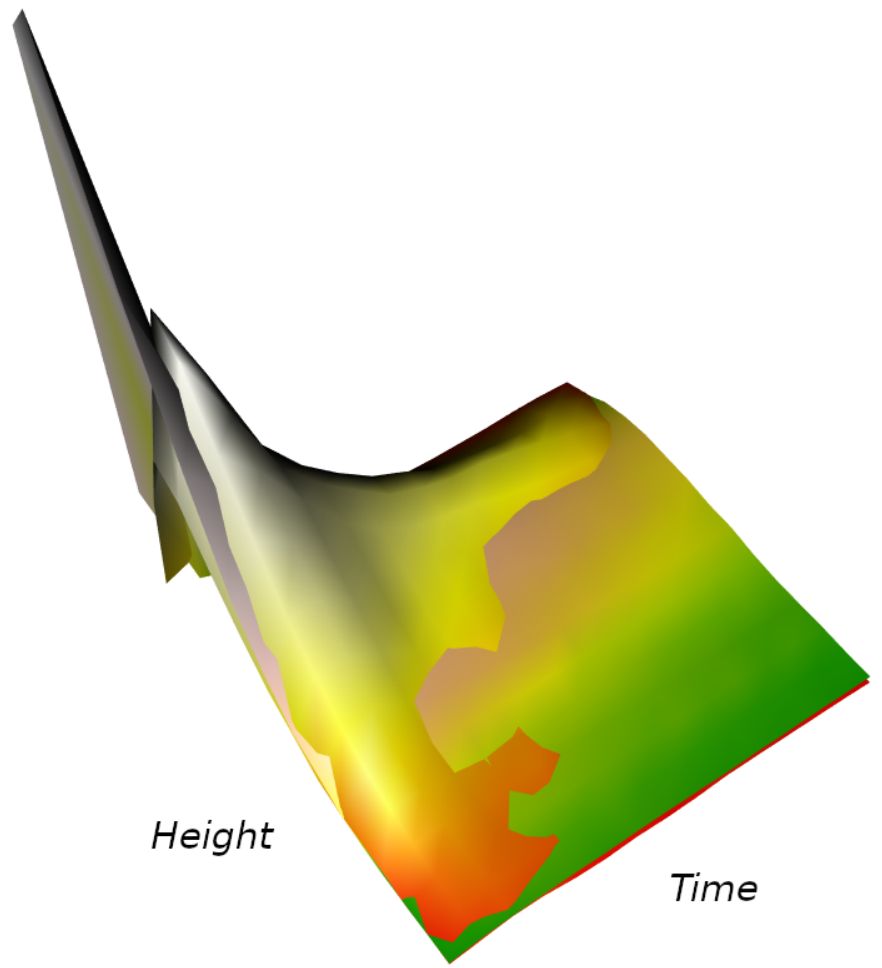

Figure C.4: Contained Smoothed Naive(Terrain) vs Triangle (Heat) 


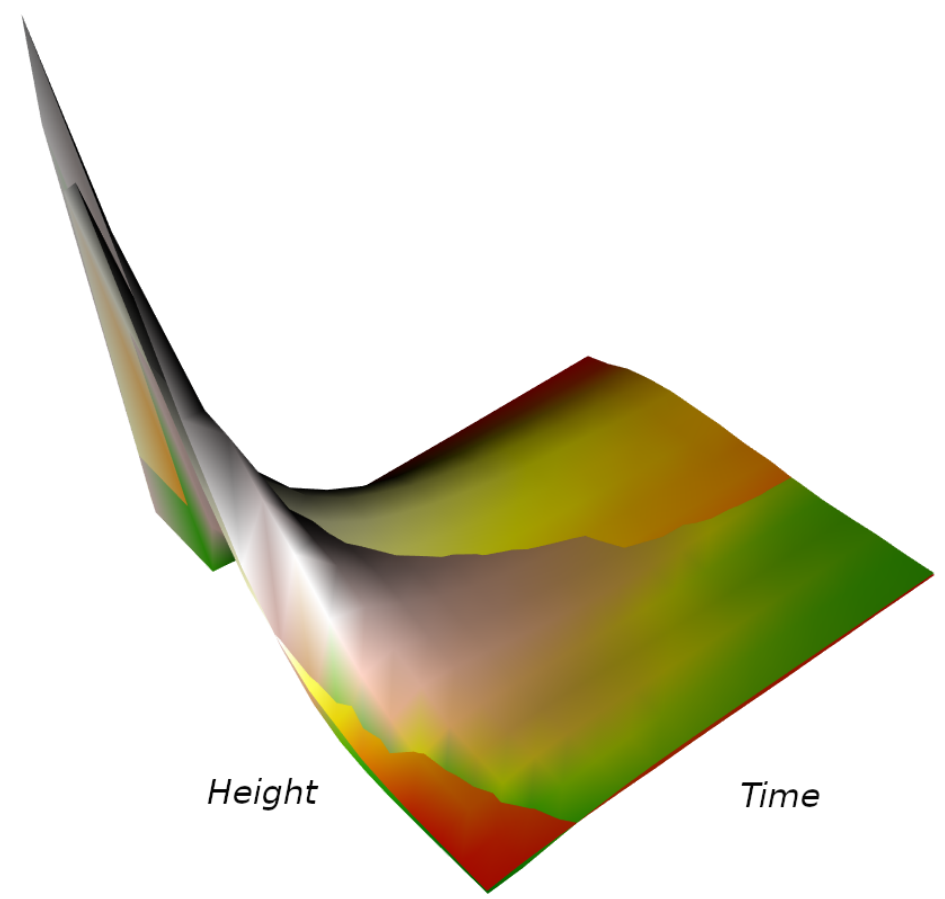

Figure C.5: TBot Up Constrained Smoothed Naive(Heat) vs Brownian Motion (Terrain) $\sigma=0.173$

of the $h$ and $\tau$ values that are of most interest. This is not easily identifiable in print as opposed to a 3D plot navigated on a computer screen, experimenting with numerous parameter values shows the raw Brownian Motion model is unlikely to fit well.

On some thought and considering other derivations of continuous time stochastic processes first passage times, it seems unlikely and certainly quite difficult to try and establish a structural process which will model the data well. Overwhelmingly the literature implies that continuous time process which are extrapolated from periodic returns boiled down to continuous time provides a potential fit. At a coarse intuitive level the Naive trades do seem to visually match the Brownian Motion fit as can be seen by comparing with the Euler estimation, but the Triangle trades certainly do not. It appears the intensity of arrival time is different between the Naive and Triangle cases, this may mean that the underling processes are not the same and hence difficult to compare. It is hard to conclude from this but given the complexity of the first passage time distribution objects it seems wise to investigate other methods using a more statistical approach first.

There is a curious effect that may be embedded in the data that relates to fat tails often observed in periodic returns. In a sense the returns modelled are inverted in that the time component varies but the observed return is a fixed quantity of 1 and -1 . This means that the previous fat tails will now be embedded in the distribution head. This is a pivotal point of reasoning which helps to fit the Intensity based model. 


\section{C.4 Intensity Approach}

The difficulties of the Structural approach motivated using simpler modelling alternatives and the result of this is the Intensity approach. The Intensity approach is to simply consider the wins and losses as binary 1 or -1 values and model the intensity of arrival times as exponential terms using the idea that arrival times can be viewed as a non-homogeneous Poisson process. At first this seems an overly simplistic comparison, but it enables a contrast between Triangle and Naive approaches that is quite clear without complex first passage distribution derivations. Also an intensity approach provides access to a variety of statistical tests and visual comparisons that are of direct practical use.

With the Intensity based approach several distributions, for example Gumbel, were initially injected with the intention of fitting across each win and loss surface of stopping times; however, it was not possible to fit these across the entire 2D surface and eventually the method settled on was the GEV fit described here across section of $h$ values. Alternative methods are discussed in 8.1 and only the final GEV method comparison of the decision points going forward are covered in this trial. The wider trial consisted of a more varied approach but I only mention many of these briefly in discussion due to their lack of value. Unfortunately with GEV, partly to do with the exponentials in the distribution, fitting over the entire surface invariably either produces a particularly poor fit or results in errors or a lack of convergence. With simple distributions it is generally less likely to get errors or a lack of convergence, but it is particularly difficult to fit the long right tail - this often involving several years with US equities. One choice would have been to not include the long tails but this discounts a reasonably large proportion of the sample, likely resulting in biased estimates and implying an ability to see into the future.

The intuition break in developing this model came in realising that the traditional problem of periodic return fat tails being difficult to model had been inverted and that the previous tails were instead within the head of the distribution which now primarily resided on the left of the distribution. The answer then seemed obvious in that the need was to predict the possibility of the extreme event of no events occurring for a long time to which there are a range of extreme value theorem solutions from which GEV was selected.

Using GEV as a sub-distribution is flexible such that it allows a close fit of the tail such that the Cumulative Differences test produces acceptably high p-values for in-sample data. It is worth stating that many other Intensity based tests do not give such high pvalues, the Cumulative Differences is chosen for convenience but this does highlight the precariousness of selective testing. This test is useful in that it reflects the closeness of fit that can be seen visually enough to present a numerical value for goodness of fit. The 
fit is much closer than Brownian Motion due to the degree of flexibility embedded in the GEV distribution. No other distribution fitted remotely as close.

Win PDF

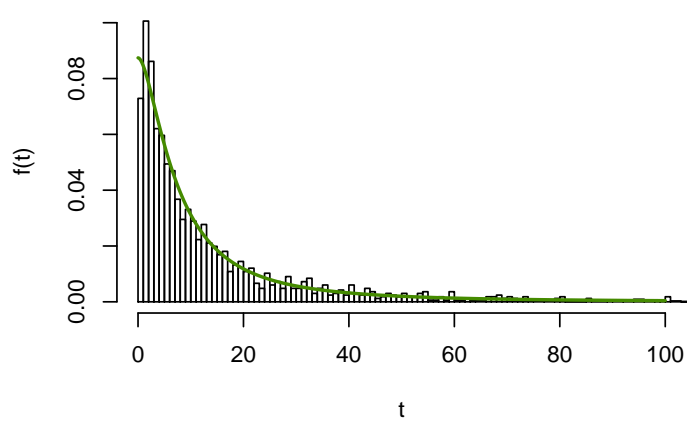

Win CDF

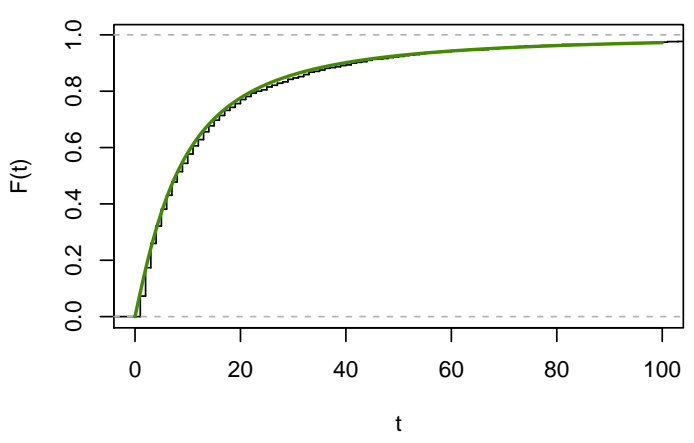

Loss PDF

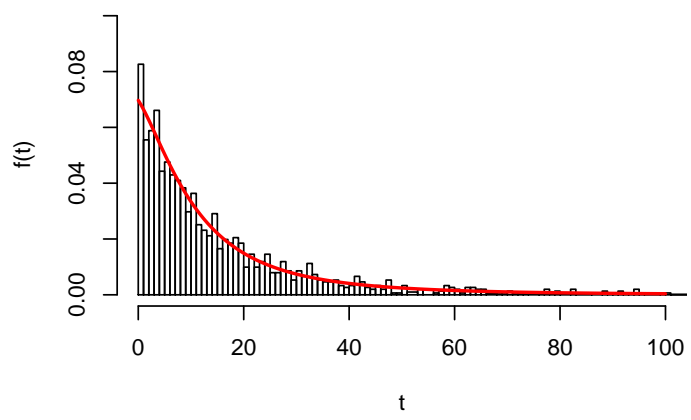

Loss CDF

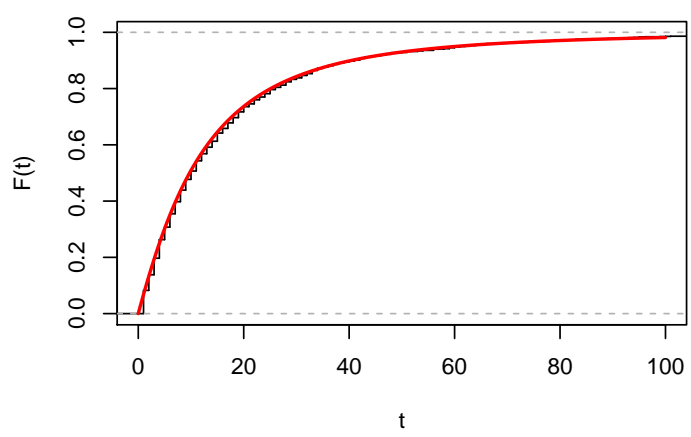

Figure C.6: TBot Up Intensity Distribution Profile $h_{\sigma}=[0,0.0035)$

Due to difficulty fitting across the entire surface, $h$ sections were trialled where slices of $h$ intervals are fitted using the GEV intensity model. Too many intervals being unmanageable, intervals are chosen to represent high, medium and low height sections. However the fit is not always particularly good so this was further normalised by dividing by $\sigma$ to create $h_{\sigma}=\frac{h}{\sigma}$ sections, this also being later useful to normalise comparison of stocks. In this trial volatility is set to $\sigma=0.173$, though this is data-snooping this is only meant as test the usefulness of the model. $h_{\sigma}$ sections show better GEV Intensity model fits as seen in Table C.14 as compared with Table C.11.

In considering the experimentation with various surface plots and the difficulty interpreting them, particularly in document form, I instead utilise the $h_{\sigma}$ slices to produce a 'distribution profile' plot across wins and losses to compare model and empirical results as seen in Figures C.6 and C.7. These can be either proportional to show the effect of probability of winning and stopping time distribution or full to only show the stopping time distributions. As a simplification since the model fit is generally exceptionally close to the in-sample empirical data, when comparing out-of-sample 
Win PDF

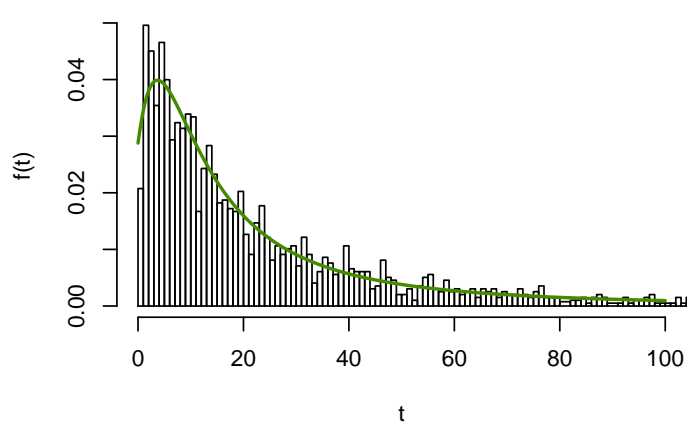

Win CDF

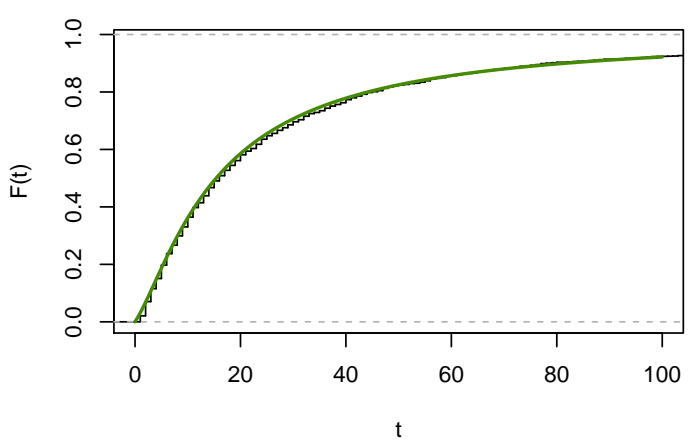

Loss PDF

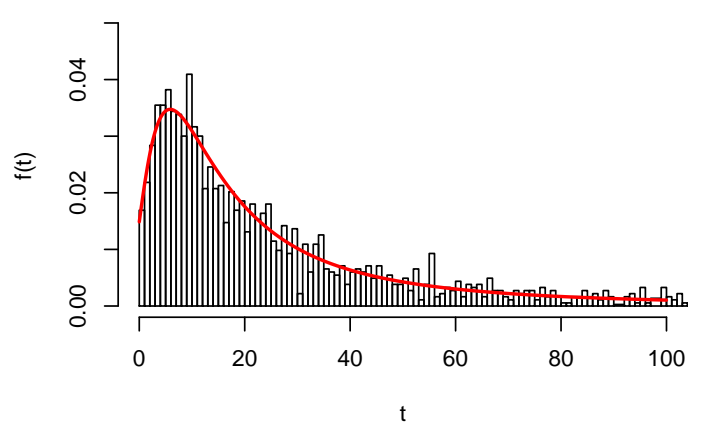

Loss CDF

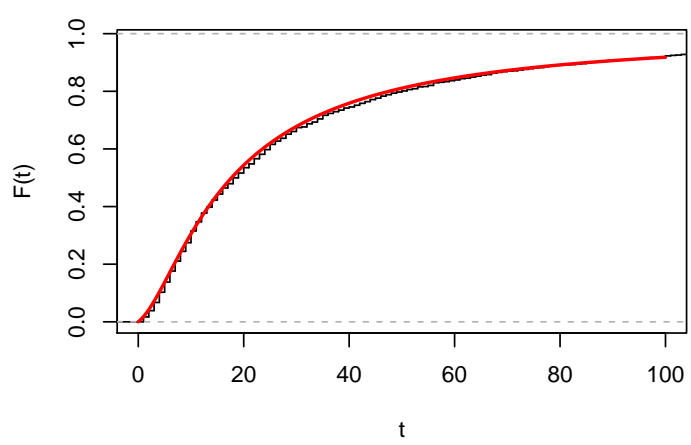

Figure C.7: TBot Up Intensity Distribution Profile $h_{\sigma}=[0.0035,007)$

empirical distributions or against the Naive case the model fit is used as the base comparison to represent the in-sample data - this default considerably lessens the number of diagrams and tests.

Table C.10: GBPUSD $h$ Intensity Section Counts

\begin{tabular}{lll}
\hline Type & $h$ & Count \\
\hline TTop Up & {$[0.00075)$} & 4301 \\
TTop Up & {$[.00075 .0015)$} & 3844 \\
TTop Up & {$[.0015 \infty)$} & 2464 \\
TTop Down & {$[0.00075)$} & 3304 \\
TTop Down & {$[.00075 .0015)$} & 2877 \\
TTop Down & {$[.0015 \infty)$} & 1763 \\
TBot Down & {$[0.00075)$} & 4034 \\
TBot Down & {$[.00075 .0015)$} & 3779 \\
TBot Down & {$[.0015 \infty)$} & 2439 \\
TBot Up & {$[0.00075)$} & 4268 \\
TBot Up & {$[.00075 .0015)$} & 3691 \\
TBot Up & {$[.0015 \infty)$} & 2129 \\
\hline
\end{tabular}


Table C.11: GBPUSD $h$ Intensity Model Parameter Estimates

\begin{tabular}{lllllllllll}
\hline & & \multicolumn{3}{c}{ Win Distribution } & & \multicolumn{3}{c}{ Loss Distribution } & \\
Type & $h$ & $\mu_{1}$ & $\sigma_{1}$ & $\xi_{1}$ & & $\mu_{2}$ & $\sigma_{2}$ & $\xi_{2}$ & $p$ \\
\hline TTop Up & {$[0.00075)$} & 4.17 & 6.9 & 0.584 & & 5.96 & 8.43 & 0.509 & 0.514 \\
TTop Up & {$[.00075 .0015)$} & 10.3 & 12.4 & 0.902 & & 16.2 & 16.7 & 0.763 & 0.511 \\
TTop Up & {$[.0015 \infty)$} & 12.7 & 31.1 & 0.963 & & 20 & 47.3 & 0.848 & 0.5 \\
TTop Down & {$[0.00075)$} & 4.25 & 6.96 & 0.628 & & 4.45 & 9.14 & 0.502 & 0.526 \\
TTop Down & {$[.00075 .0015)$} & 11.5 & 13.8 & 0.794 & & 15.3 & 16.5 & 0.845 & 0.53 \\
TTop Down & {$[.0015 \infty)$} & 15 & 31.5 & 0.848 & & 30.7 & 36.1 & 0.99 & 0.55 \\
TBot Down & {$[0.00075)$} & 4.37 & 6.15 & 0.637 & & 6.62 & 8.17 & 0.614 & 0.513 \\
TBot Down & {$[.00075 .0015)$} & 9.66 & 12.8 & 0.87 & & 15.7 & 16.3 & 0.721 & 0.518 \\
TBot Down & {$[.0015 \infty)$} & 3.1 & 27.4 & 0.954 & & 0.713 & 41.7 & 0.75 & 0.501 \\
TBot Up & {$[0.00075)$} & 3.18 & 7.1 & 0.627 & & 3.82 & 8.82 & 0.496 & 0.518 \\
TBot Up & {$[.00075 .0015)$} & 10.9 & 14.2 & 0.799 & & 14.4 & 16.1 & 0.744 & 0.525 \\
TBot Up & {$[.0015 \infty)$} & 3.95 & 27.5 & 0.957 & & 1.74 & 40.6 & 0.834 & 0.53 \\
\hline
\end{tabular}

Table C.12: GBPUSD $h$ Triangle Intensity Model In Sample Tests

\begin{tabular}{|c|c|c|c|}
\hline \multirow[b]{2}{*}{ Type } & \multirow[b]{2}{*}{$h$} & \multirow{2}{*}{$\begin{array}{l}\text { Win Distribution } \\
p G_{T 1}(t)\end{array}$} & \multirow{2}{*}{$\frac{\text { Loss Distribution }}{(1-p) G_{T 2}(t)}$} \\
\hline & & & \\
\hline TTop Up & [0.00075) & 0.495 & 0.48 \\
\hline TTop Up & [.00075 .0015) & 1 & 0.843 \\
\hline TTop Up & {$[.0015 \infty)$} & 0.0509 & 0.362 \\
\hline TTop Down & [0 .00075) & 0.539 & 0.523 \\
\hline TTop Down & [.00075.0015) & 0.854 & 0.99 \\
\hline TTop Down & {$[.0015 \infty)$} & 0.0492 & 0.00247 \\
\hline TBot Down & [0 .00075) & 0.87 & 0.858 \\
\hline TBot Down & [.00075.0015) & 0.452 & 0.35 \\
\hline TBot Down & {$[.0015 \infty)$} & 0.0335 & 0.208 \\
\hline TBot Up & [0.00075) & 0.488 & 0.477 \\
\hline TBot Up & [.00075.0015) & 0.839 & 0.74 \\
\hline TBot Up & {$[.0015 \infty)$} & 0.59 & 0.126 \\
\hline
\end{tabular}

Table C.13: GBPUSD $h_{\sigma}$ Intensity Section Counts

\begin{tabular}{lll}
\hline Type & $h_{\sigma}$ & Count \\
\hline TTop Up & {$[0.0035)$} & 3181 \\
TTop Up & {$[.0035 .007)$} & 3873 \\
TTop Up & {$[.007 \infty)$} & 3555 \\
TTop Down & {$[0.0035)$} & 2436 \\
TTop Down & {$[.0035 .007)$} & 2943 \\
TTop Down & {$[.007 \infty)$} & 2565 \\
TBot Down & {$[0.0035)$} & 2966 \\
TBot Down & {$[.0035 .007)$} & 3814 \\
TBot Down & {$[.007 \infty)$} & 3472 \\
TBot Up & {$[0.0035)$} & 3173 \\
TBot Up & {$[.0035 .007)$} & 3808 \\
TBot Up & {$[.007 \infty)$} & 3107 \\
\hline
\end{tabular}


Table C.14: GBPUSD $h_{\sigma}$ Intensity Model Parameter Estimates

\begin{tabular}{lllllllllll}
\hline & & \multicolumn{3}{c}{ Win Distribution } & & \multicolumn{3}{c}{ Loss Distribution } & \\
Type & $h_{\sigma}$ & $\mu_{1}$ & $\sigma_{1}$ & $\xi_{1}$ & & $\mu_{2}$ & $\sigma_{2}$ & $\xi_{2}$ & $p$ \\
\hline TTop Up & {$[0.0035)$} & 2.52 & 6.45 & 0.519 & & 5.3 & 7.48 & 0.489 & 0.513 \\
TTop Up & {$[.0035 .007)$} & 8.6 & 10.1 & 0.786 & & 13.1 & 13.9 & 0.661 & 0.51 \\
TTop Up & {$[.007 \infty)$} & 15 & 26.7 & 0.99 & & 20 & 39.1 & 0.836 & 0.507 \\
TTop Down & {$[0.0035)$} & 0.5 & 6.52 & 0.49 & & 3 & 7.72 & 0.491 & 0.534 \\
TTop Down & {$[.0035 .007)$} & 9.57 & 11.2 & 0.764 & & 10 & 14.8 & 0.6 & 0.523 \\
TTop Down & {$[.007 \infty)$} & 10 & 28.4 & 0.849 & & 28.4 & 34 & 0.99 & 0.539 \\
TBot Down & {$[0.0035)$} & 3.29 & 5.76 & 0.527 & & 5.65 & 7.45 & 0.536 & 0.517 \\
TBot Down & {$[.0035 .007)$} & 7.9 & 10.4 & 0.793 & & 10 & 14.4 & 0.596 & 0.514 \\
TBot Down & {$[.007 \infty)$} & 15 & 26.9 & 0.99 & & 30.2 & 35.7 & 0.933 & 0.505 \\
TBot Up & {$[0.0035)$} & 2.57 & 6.19 & 0.628 & & 0.5 & 8.25 & 0.4 & 0.523 \\
TBot Up & {$[.0035 .007)$} & 9.15 & 12.1 & 0.732 & & 11.7 & 13.2 & 0.701 & 0.519 \\
TBot Up & {$[.007 \infty)$} & 15 & 27.4 & 0.967 & & 16.8 & 38.9 & 0.835 & 0.527 \\
\hline
\end{tabular}

Table C.15: GBPUSD $h_{\sigma}$ Triangle Intensity Model In Sample Tests

\begin{tabular}{lllll}
\hline & & Win Distribution & & Loss Distribution \\
Type & $h_{\sigma}$ & $p G_{T 1}(t)$ & & $(1-p) G_{T 2}(t)$ \\
\hline TTop Up & {$[0.0035)$} & 0.667 & 0.658 \\
TTop Up & {$[.0035 .007)$} & 0.98 & 0.966 \\
TTop Up & {$[.007 \infty)$} & 0.39 & 0.984 \\
TTop Down & {$[0.0035)$} & 0.775 & 0.76 \\
TTop Down & {$[.0035 .007)$} & 0.26 & 0.263 \\
TTop Down & {$[.007 \infty)$} & 0.5 & 0.125 \\
TBot Down & {$[0.0035)$} & 0.493 & 0.478 \\
TBot Down & {$[.0035 .007)$} & 0.764 & 0.71 \\
TBot Down & {$[.007 \infty)$} & 0.151 & 0.597 \\
TBot Up & {$[0.0035)$} & 0.951 & 0.946 \\
TBot Up & {$[.0035 .007)$} & 0.565 & 0.591 \\
TBot Up & {$[.007 \infty)$} & 0.779 & 0.296 \\
\hline
\end{tabular}

\section{C.5 Discussion}

For the Brownian Motion first passage approach, there are various problems with this and while many of these certainly could be resolved, my eventual conclusion is that this methodology is overly complex for the potential gains in information. Instead from observing both the first passage surfaces it seemed the binary returns nature of the data presented a simple alternative, it seemed better to at least start by not assuming a structure but rather create a model into which various distributions can be injected. Under this more exploratory view, very little needs to be assumed and the fit can be adjusted until the a distribution is found. This is how the Intensity model was developed as shown.

Critically I view much of the financial literature as being overly confident in their 
mathematical models with suspiciously derived empirical validation; Breiman (2001b) has some interesting discussion points on the over-reliance of data models, my response is to very carefully explore potential fits on similar data (GBPUSD), then clearly knowing I can analysis momentum well, apply the knowledge to Equity prices, while not data-snooping. By abstracting prediction cleanly to an algorithm I avoid unrealistic model prediction assumptions and only need to rely on comparative models to show differences, thus reducing over reliance on mathematical models. In the end the intensity model was used and eventually the GEV distribution was chosen to model the underlying momentum within this. The model being realistically developed to make this comparison it can now be adjusted and applied to US Equities confidently.

Exploration of Brownian Motion based first passage times is somewhat opaque since it requires considerable derivation to explore various approaches properly without a clear initial idea of how the model may fit. This makes exploring such methods time consuming and problematic to find well fitted parameters to for even simple Itô processes. Also, I see no comparative research extracting information from such a method for comparing price momentum, none in terms of short term momentum using first passage times. Despite first passage times being thoroughly investigated it is the absence of information generated by highly skilled mathematicians in this area that concerns me. The case of no barking dog in the night, where there are many capable a bark is a concern, providing clue that this is rather difficult. Colloquially if the general population of highly skilled mathematicians have not found valuable information in such an area to publish, then I strongly question my ability to do this within the time frame required of a Masters thesis.

For the structural parameter estimations, the results show that the volatility parameters give indicative changes but that the drift parameters are very close to zero. The drift values of zero look suspicious and yet it is likely that the drift is close to zero over the short term. The question then is how to validate the accuracy of these values and how to test for significance. Categorical Chi squared testing can be used to test grid sections of probability but on trialling this, it was apparent that the results are entirely dependent on the size of the $h$ by $\tau$ grid squares, making this an unreliable comparative test since it can be adjusted to give whatever p-values seem desirable! Overall the approach to testing structural models is unclear in this situation, and given other problems this was not investigated further.

Estimates of volatility for Brownian Motion first passage times give some idea of changes potentially due to Triangle events and these seem to corroborate with measured bet returns. This is interesting especially since these are quite different from the Naive case which shows similar volatility to the Euler estimation, but it is not clear how significant this change is. To explore the meaning of parameters I plot the constrained surfaces of first passage responses for Triangles, Naive and the Euler estimation with no 
drift (though for clarity and space only TBot Up is shown, since all cases are similar). Arguably the most interesting result of this is that there seems to be a much greater number of early first passage times toward the greater $h$ values, than there are with the Naive or Euler estimation cases. This can be seen clearly in Figure C.4 where the orange rise shows a larger number of early stopping times in this region. There are two potential aspects to explore within this: there may be some more immediate change due to Triangle events and that this may be time dependant, in that the effect could be stronger closer to the Triangle than further away. This is a concern since using time varying functions as given in 4.1 could be exceptionally complex to derive first passage times. It is also worth noting that by substituting estimated parameters into the Brownian Motion based surface it is very clear that the estimated parameters while indicatively higher do not represent the underlying empirical data well. This is likely ideally dealt with by using a time varying Itô process or a Levy process with jumps, either of which would be particularly difficult to derive the first passage distribution for, since in moving towards equities this would also likely require a Geometric approach. So the Itô based first passage time method would probably require considerably more work to obtain a model that fitted.

Though the structural first passage is an entry point trial, its main weakness is that it discards roughly half the sample of Triangle events and this sample may misrepresent the real effect. The sample captured represents only those that have crossed within 60 minutes or less. To some extent this can be mitigate with crossing times above and below.

For the Itô Stochastic process general case, the issues going forward are:

- complex to the point where if for example the modelling requires a time varying function there may not be a solution,

- by definition implies a very specific structure which may be impractical,

- the intricate nature of derivation makes adding covariates convoluted,

- may be difficult to test for significance of effect,

- unclear how to validate if estimates are correct (aside from visually).

The alternative is to not assume structure and consider the bet returns as simple exponential stopping times based on the intensity method from survival analysis. Mathematically this is shown in Section 5.1 where the exponential stopping time based motivation is shown. This allows the ability to trial various distributions to model the stopping time while also allowing the flexibility to add covariates. End the end this has resulted in the GEV distribution, but the point is the overall solution is particularly malleable.

Using GBPUSD some preliminary results are shown, while it would have been conceptually better to fit across the entire surface of stopping times, fitting across three sections considerably simplifies assessment of the results. The fit assessed using the Cumulative Deviations test is generally good, but somewhat unstable if only done using 
$h$, this is also not usable for Equities. Instead I use $h_{\sigma}=\frac{h}{\sigma}$ to normalise the data and this produces more consistent goodness of fit tests in the trial.

To intuitively view Intensity fit I create a 'Profile Plot' which contains the win PDF and CDF as a green line vs win EPDF and ECDF in the top half; and loss PDF and CDF as a red line vs loss EPDF ECDF. PDF vs EPDF densities are normalised by frequency for comparison purposes such that histogram bars are comparable with the model line fit. As can be seen by the profile plots and goodness of fit tests the the fits are surprisingly good (even better with Equities when volatility is calculated on a case by case basis).

Originally this intensity model had not worked as intended, as none of the distributions I tried fitted and it seemed a dead end. This was blind; however upon reading McNeil et al. (2015) regarding risk management I realised my strategy was entirely flawed in that in trying to predict the head on the left of profile plots was highly unlikely to be achieved in that it was unstable and potentially unpredictable. However in reading the risk management literature I realised that the heavy tail that risk managers complain as being unpredictable was now constrained on the left of my plots, and though this was still apparently difficult to model the tail on the right was now quite predictable or at least easily modelable. Through the process of taking changing risk to use fixed price levels the nature of the problem had changed. It was apparent that trying to predict the head was of no use but that by predicting the tail the head was predictable by inversion: $p_{\text {head }}=1-p_{\text {tail }}$. Thus the original tail problem was solvable, if only for a narrow case. From Extreme Value Theory I originally trialled Gumbel but found GEV provided a more flexible fit.

As can be seen in Figures C.7 and C.6 the left of the PDF vs EPDF plots generally do not fit well but the tails do. This may be because the effect of the Triangles is more extreme immediately after the Triangle, whereas the tail is less affected by the Triangle and so is more predictable. Or perhaps more obviously in that the GEV fit is not able to take into account the frequent more immediate change as seen on the left of the plot. This is explored more for US Equities where I discuss the GEV intensity model predictive value. 


\section{Appendix D}

\section{ES Futures Trial}

S\&P 500 CME Group Futures (ES) data from Thompson Reuters over the period of 20042017 inclusive is used as secondary trial data. This data uses the standard first monthly aggregated continuous contract reflective of the 3 month ES contracts contracts rolled over, which is the common standard for back-testing futures. The Bid price is used as the quoted price. Transaction costs only take into Account the Bid Ask spread and estimate brokerage costs as an additional fixed spread.

Originally this data had intended to be used with Geometric Brownian motion or other continuous time structural stochastic processes. The ES data has a geometric drift component which would have been a useful trial transition to stocks for such models. However in the end this data was not used for this purpose, and so little emphasis has been placed on this trial.

What is interesting is that data shows a strong predictive return component after Triangles however this drastically reduces once transaction costs are applied.

Table D.1: ES Triangle Counts

\begin{tabular}{lrrrr}
\hline & TTop Up & TBot Down & TTop Down & TBot Up \\
\hline Count & 7500 & 6862 & 5466 & 6543 \\
Count, $h>5$ & 427 & 455 & 302 & 399 \\
\hline
\end{tabular}

Table D.2: ES Triangle Mean Returns

\begin{tabular}{lrrrr}
\hline & TTop Up & TBot Down & TTop Down & TBot Up \\
\hline Raw Return (no costs) & 0.1490 & 0.108000 & 0.1720 & 0.1870 \\
Raw Return (no costs), $h>5$ & 0.0804 & 0.054800 & 0.1420 & 0.1010 \\
Real Return (with costs), $h>5$ & 0.0256 & 0.000105 & 0.0878 & 0.0461 \\
\hline
\end{tabular}


Table D.3: ES Triangle Mean Return T-test P-Values

\begin{tabular}{lrrrr}
\hline & TTop Up & TBot Down & TTop Down & TBot Up \\
\hline Raw Return $=0$ & 0.000 & 0.000 & 0.0000 & 0.0000 \\
Raw Return $>0$ & 0.000 & 0.000 & 0.0000 & 0.0000 \\
Real Return $>0$ & 0.218 & 0.499 & 0.0110 & 0.0776 \\
Real Return $>.01$ & 0.318 & 0.617 & 0.0211 & 0.1330 \\
\hline
\end{tabular}




\section{Appendix E}

\section{Effect Size Heuristics Bootstrap Code}

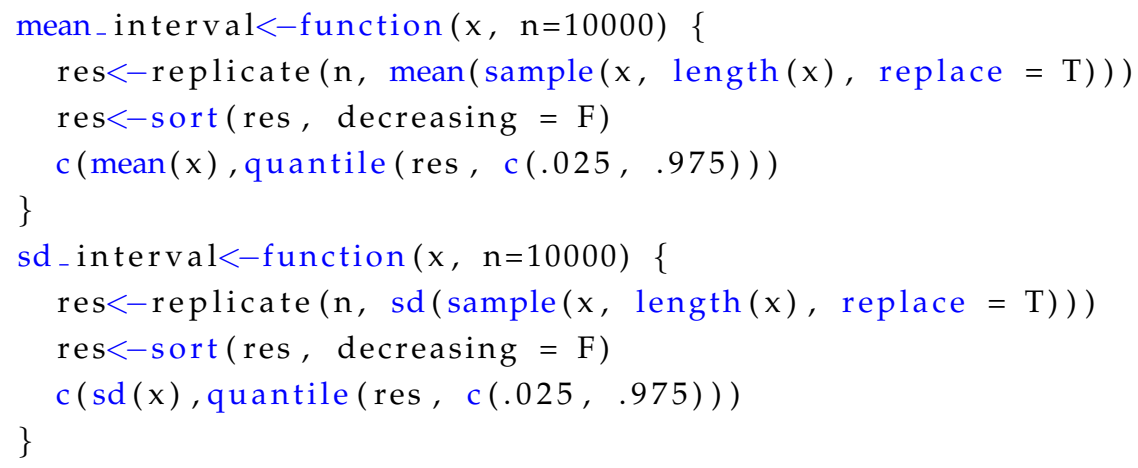

Listing E.1: Mean and Standard Deviation Confidence Interval Bootstrap

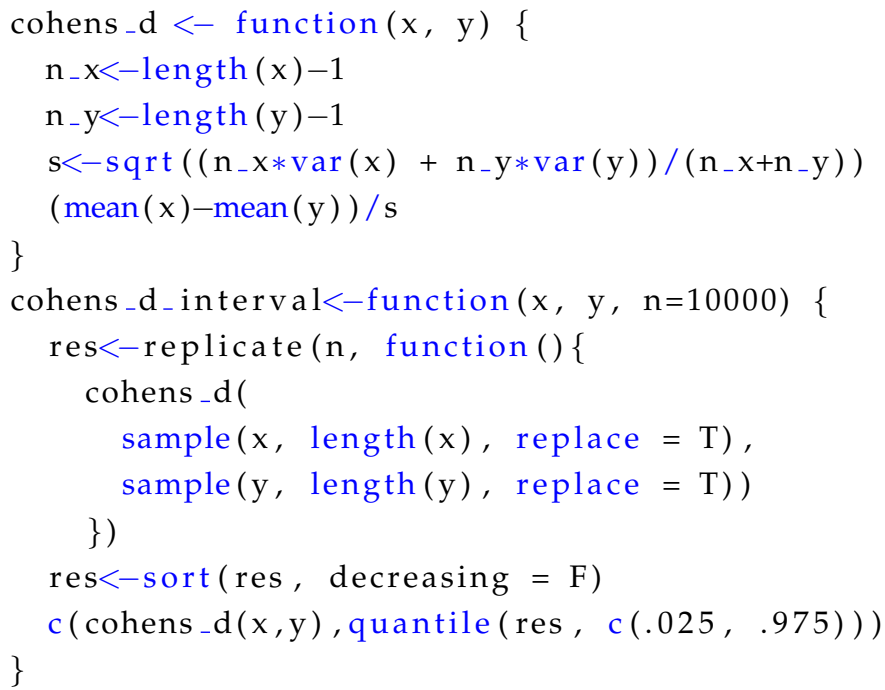

Listing E.2: Cohen's d Confidence Interval Bootstrap 


\section{Bibliography}

Ahlawat, S. (2015). Probabilistic neural networks for identification and empirical evaluation of technical analysis-based forecasting. Wilmott, 2015(79):38-49.

Bailey, D., Borwein, J. J., Lopez de Prado, M., and Zhu, Q. (2014). Pseudo-mathematics and financial charlatanism: The effects of backtest overfitting on out-of-sample performance. 61:458.

Bedell, K. (2006). Opinions on opinionated software. Linux J., 2006(147):1-.

Borodin, A. N. and Salminen, P. (2002). Handbook of Brownian motion: facts and formulae. Springer.

Brant, P. L. (2011). Diary of a professional commodity trader: Lessons from 21 weeks of real trading. book.

Breiman, L. (2001a). Statistical modeling: The two cultures. Statistical Science.

Breiman, L. (2001b). Statistical modeling: The two cultures. Statistical Science, 16(3):199_ 231.

Buckland, S. T. (1984). Monte carlo confidence intervals. Biometrics, 40(3):811-817.

Carver, R. (2015). Systematic Trading: A unique new method for designing trading and investing systems. EBL-Schweitzer. Harriman House Limited.

Chan, E. (2009). Quantitative Trading: How to Build Your Own Algorithmic Trading Business. Wiley Trading. Wiley.

Chan, E. (2013). Algorithmic Trading: Winning Strategies and Their Rationale. Wiley Publishing, 1st edition.

Chan, E. (2016). Machine Trading: Deploying Computer Algorithms to Conquer the Markets. Wiley Trading. Wiley.

Connor, E. F. and Simberloff, D. (1986). Competition, scientific method, and null models in ecology: Because field experiments are difficult to perform, ecologists often rely on evidence that is nonexperimental and that therefore needs to be rigorously evaluated. American Scientist, 74(2):155-162. 
Cumming, G. (2012). Understanding the New Statistics: Effect Sizes, Confidence Intervals, and Meta-analysis. Multivariate applications book series. Routledge.

Duffie, D. (2010). Dynamic Asset Pricing Theory: Third Edition. Princeton Series in Finance. Princeton University Press.

Escobar, A., Moreno, J., and Múnera, S. (2013). A technical analysis indicator based on fuzzy logic. Electronic Notes in Theoretical Computer Science, 292:27 - 37. Proceedings of the XXXVIII Latin American Conference in Informatics (CLEI).

Fama, E. (1970). Efficient capital markets: A review of theory and empirical work. Journal of Finance, 25:383-417.

Fama, E. (1998). Market efficiency, long-term returns, and behavioral finance. Journal of Financial Economics, 49(3):283-306.

Fama, E. F. (1965). The behavior of stock-market prices. Journal of Business, 38(1):34-105.

Fama, E. F. (1991). Efficient capital markets II. Journal of Finance, 46(5):1575-1617.

Fama, E. F. and French, K. R. (1988). Permanent and Temporary Components of Stock Prices. The Journal of Political Economy, 96(2):246-273.

Fama, E. F. and French, K. R. (1996). Multifactor explanations of asset pricing anomalies. The Journal of Finance, 51(1):55-84.

Frankfurter, G. M. and McGoun, E. G. (2002). Resistance is futile: the assimilation of behavioral finance. Journal of Economic Behavior and Organization, 48(4):375-389.

Harvey, C. R., Liu, Y., and Zhu, H. (2016). ... and the cross-section of expected returns. The Review of Financial Studies, 29(1):5-68.

Hudson, R. and Gregoriou, A. (2015). Calculating and comparing security returns is harder than you think: A comparison between logarithmic and simple returns. International Review of Financial Analysis, 38(C):151-162.

Jegadeesh, N. and Titman, S. (1993). Returns to Buying Winners and Selling Losers: Implications for Stock Market Efficiency. The Journal of Finance, 48(1):65-91.

Kahneman, D. (2011). Thinking, fast and slow. Farrar, Straus and Giroux, New York.

Kalbfleisch, J. D. and Prentice, R. L. (2002). The Statistical Analysis of Failure Time Data. John Wiley \& Sons, 2nd edition.

Karatzas, I. and Shreve, S. (1988). Brownian motion and stochastic calculus. Graduate texts in mathematics. Springer-Verlag.

Kloeden, P. E. and Platen, E. (1992). Numerical Solutions of Stochastic Differential Equations. Springer-Verlag, Berlin. 
Lo, A. (2017). Adaptive Markets: Financial Evolution at the Speed of Thought. Princeton University Press.

Lo, A. and MacKinlay, A. (1999). A non-random walk down Wall Street. Princeton Univ. Press, Princeton, NJ [u.a.].

Lo, A. W., Mamaysky, H., and Wang, J. (2000). Foundations of technical analysis: Computational algorithms, statistical inference, and empirical implementation. Journal of Finance, 40:1705-1765.

Malkiel, B. G. (2003). A Random Walk Down Wall Street, Completely Revised and Updated Edition. W. W. Norton \& Company.

Mandelbrot, B. and Hudson, R. L. (2004). The (mis) Behaviour of Markets: A Fractal View of Risk, Ruin and Reward. Profile Books.

Manly, B. F. J. (2007). Randomization, Bootstrap and Monte Carlo Methods in Biology. Chapman \& Hall.

McNeil, A. J., Frey, R., and Embrechts, P. (2015). Quantitative Risk Management: Concepts, Techniques and Tools. Princeton University Press, Princeton, NJ, USA.

Murphy, J. J. and Murphy, J. J. (1999). Technical analysis of the financial markets. New York Institute of Finance, Fishkill, N.Y.

Osler, C. (2000). Support for resistance: technical analysis and intraday exchange rates. Economic Policy Review, (Jul):53-68.

Osler, C., Chang, P., and of New York, F. R. B. (1995). Head and shoulders: not just a flaky pattern. Staff reports. Federal Reserve Bank of New York.

Raj, M. and Thurston, D. (1996). Effectiveness of simple technical trading rules in the hong kong futures markets. Applied Economics Letters, 3(1):33-36.

Schwab, A., Abrahamson, E., Starbuck, W. H., and Fidler, F. (2011). Perspectiveresearchers should make thoughtful assessments instead of null-hypothesis significance tests. Organization Science, 22(4):1105-1120.

Taylor, S. J. (2011). Asset Price Dynamics, Volatility, and Prediction. Princeton University Press.

Thaler, R., editor (2005). Advances in behavioral finance. The roundtable series in behavioral economics. Princeton Univ. Press [u.a.], Princeton, NJ [u.a.].

Tharp, V. (2008). The Definitive Guide to Position Sizing: How to Evaluate Your System and Use Position Sizing to Meet Your Objectives. International Institute of Trading Mastery. 
Thorp, E. (2017). A Man for All Markets: Beating the Odds, from Las Vegas to Wall Street. Oneworld Publications.

Yu, P. L., Lam, K., and Ng, S. H. (2011). Automating Technical Analysis, pages 85-96. World Scientific. 